Supporting Information

\title{
Rare-Earth-Catalyzed Selective 1,4-Hydrosilylation of Branched 1,3-Enynes Giving Tetrasubstituted Silylallenes
}

Wufeng Chen, Chunhui Jiang, Jianying Zhang, Jiaqi Xu, Lin Xu, Xiufang Xu, Jianfeng Li* and Chunming Cui*

State Key Laboratory of Elemento-Organic Chemistry and College of Chemistry, Nankai University, Tianjin 300071, People's Republic of China

E-mail: lijf@ nankai.edu.cn, cmcui@ nankai.edu.cn

Homepage: http://cui.nankai.edu.cn 


\section{Table of Contents}

$\begin{array}{ll}\text { General Experimental } & \text { S3 }\end{array}$

$\begin{array}{ll}\text { DFT Calculations } & \text { S22 }\end{array}$

$\begin{array}{lr}\text { Kinetic Studies } & \text { S37 }\end{array}$

$\begin{array}{lr}\text { References } & \text { S39 }\end{array}$

$\begin{array}{ll}\text { NMR Spectra } & \text { S41 }\end{array}$

$\begin{array}{lr}\text { IR Spectrum } & \text { S75 }\end{array}$ 


\section{General Experimental}

All manipulations involving air- and moisture-sensitive complexes were carried out under an atmosphere of dry argon by using modified Schlenk line and glovebox techniques. The ${ }^{1} \mathrm{H},{ }^{13} \mathrm{C}$, and ${ }^{29} \mathrm{Si}$ NMR spectroscopic data were recorded on a Bruker AV400 spectrometer. ${ }^{2} \mathrm{H}$ NMR spectra spectroscopic data was recorded on a Bruker AV600 spectrometer without proton decoupling. GC-MS data were obtained with a Thermo Scientific TRACE 1300 \& ISQ QD system, equipped with a TG5MS column. High resolution mass spectrometric (HRMS) analyses spectrum was determined on an IonSpec FT-ICR mass spectrometer. IR spectrum was recorded in the range of $400-4000 \mathrm{~cm}^{-1}$ on a Bruker Alpha II spectrophotometer in glove box filled with Ar gas.

The solvents (THF, toluene, and $n$-hexane) were freshly distilled from sodium and degassed prior to use. $\mathrm{LY}(\mathrm{THF})_{2}(\mu-\mathrm{Cl})_{2} \mathrm{Li}(\mathrm{THF})_{2}\left(\mathrm{~L}=\operatorname{ArNC}(\mathrm{Me}) \mathrm{C}(\mathrm{Me}) \mathrm{NAr}, \mathrm{Ar}=2,6-i \mathrm{Pr}_{2} \mathrm{C}_{6} \mathrm{H}_{3}\right),{ }^{\mathrm{S} 1} \mathbf{Y C},{ }^{\mathrm{S} 2} \mathbf{1}^{\mathrm{S} 3}{ }^{\mathrm{S}},{ }^{\mathrm{S} 4} \mathbf{3}^{\mathrm{S} 4}$ and $4^{\mathrm{S} 4}$ were synthesized according to the published procedures. Hydrosilanes $(6 \mathbf{6}, 6 \mathbf{b}$ and $\mathbf{6 d}-\mathbf{h})$ were obtained by the reaction of corresponding trisiloxanes with $\mathrm{LiAlH}_{4}{ }^{\mathrm{S} 5} 1,3$-Enynes $\mathbf{7} \mathbf{b}^{\mathrm{S} 6}, \mathbf{7} \mathbf{c}^{\mathrm{S} 7}$ and $\mathbf{7 d}-\mathbf{r}$ ${ }^{\mathrm{S} 8}$ were synthesized according to the published procedure. The spectra of $7 \mathbf{b}^{\mathrm{S} 6}$ and $\mathbf{7} \mathbf{c}^{\mathrm{S} 7}$ were consistent with those in the literatures. 1,3-Enynes were dried by $\mathrm{CaH}_{2}$ and then degassed prior to use. Other chemicals were of analytical grade and were used as received.
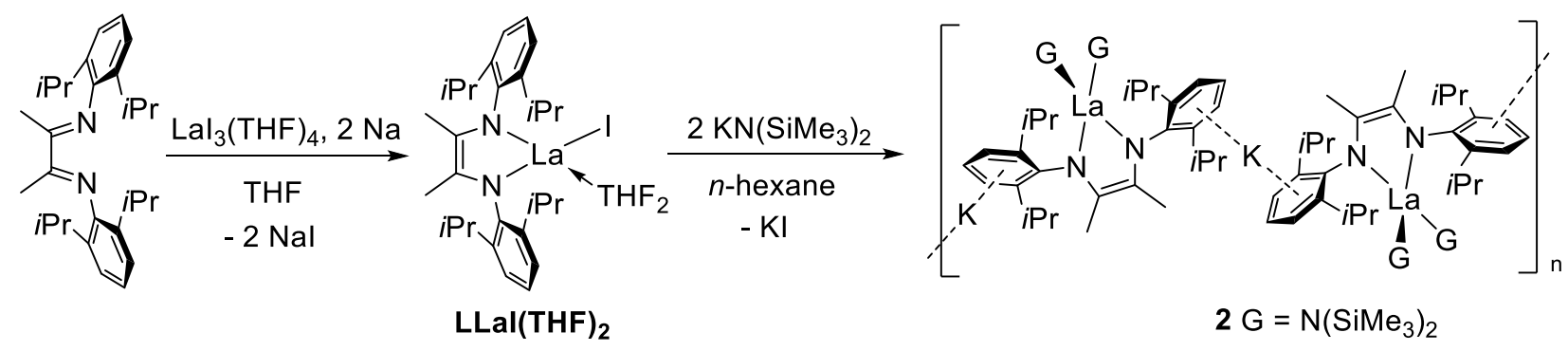

Synthesis of K[LLa(N(SiMe $\left.\left.)_{2}\right)_{2}\right]$ (2): ${ }^{\mathrm{S} 4}$ A mixture of LLaI(THF)2 (406 mg, $\left.0.5 \mathrm{mmol}\right)$ and $\mathrm{KN}\left(\mathrm{SiMe}_{3}\right)_{2}(220 \mathrm{mg}, 1.1 \mathrm{mmol})$ was added $30 \mathrm{~mL} n$-hexane. The solution was stirred at $0{ }^{\circ} \mathrm{C}$ for $8 \mathrm{~h}$. After filtration, the solid was extracted by toluene. The toluene filtrate was concentrated and kept overnight at $-35^{\circ} \mathrm{C}$ to afford red brown crystals of $2(323 \mathrm{mg}, 72 \%)$. Anal. Calcd for $\mathrm{C}_{40} \mathrm{H}_{76} \mathrm{KLaN}_{4} \mathrm{Si}_{4}$ (903.42): C, 53.18; H, 8.48; N, 6.20; Found: C, 52.69; H, 8.28; N, 6.18. ${ }^{1}$ H NMR (400 MHz, THF- $d_{8}$, $\left.23{ }^{\circ} \mathrm{C}\right) \delta 6.89\left(\mathrm{~d},{ }^{3} J_{\mathrm{HH}}=7.5 \mathrm{~Hz}, 4 \mathrm{H}, \mathrm{Ar}-H\right), 6.71\left(\mathrm{t},{ }^{3} J_{\mathrm{HH}}=7.5 \mathrm{~Hz}, 2 \mathrm{H}, \mathrm{Ar}-H\right), 3.85\left(\mathrm{br}, 4 \mathrm{H}, \mathrm{CH}\left(\mathrm{CH}_{3}\right)_{2}\right)$, $1.47\left(\mathrm{~s}, 6 \mathrm{H}, \mathrm{CH}_{3}-\mathrm{C}=\mathrm{C}-\mathrm{CH}_{3}\right), 1.26\left(\mathrm{br}, 12 \mathrm{H}, \mathrm{CH}\left(\mathrm{CH}_{3}\right)_{2}\right), 1.13\left(\mathrm{~d},{ }^{3} \mathrm{~J}_{\mathrm{HH}}=6.6 \mathrm{~Hz}, 12 \mathrm{H}, \mathrm{CH}\left(\mathrm{CH}_{3}\right)_{2}\right),-0.04$ (br, 36H, $\left.\mathrm{Si}\left(\mathrm{CH}_{3}\right)_{2}\right) .{ }^{1} \mathbf{H}$ NMR $\left(400 \mathrm{MHz}, \mathrm{THF}-d_{8},-40{ }^{\circ} \mathrm{C}\right) \delta 6.90(\mathrm{~m}, 4 \mathrm{H}, \mathrm{Ar}-H), 6.71\left(\mathrm{t},{ }^{3} J_{\mathrm{HH}}=7.5\right.$ 
$\mathrm{Hz}, 2 \mathrm{H}, \mathrm{Ar}-H), 4.00\left(\mathrm{sept},{ }^{3} \mathrm{~J}_{\mathrm{HH}}=6.4 \mathrm{~Hz}, 2 \mathrm{H}, \mathrm{CH}\left(\mathrm{CH}_{3}\right)_{2}\right), 3.62\left(\mathrm{sept},{ }^{3} \mathrm{~J}_{\mathrm{HH}}=6.4 \mathrm{~Hz}, 2 \mathrm{H}, \mathrm{CH}\left(\mathrm{CH}_{3}\right)_{2}\right)$, $1.45\left(\mathrm{~s}, 6 \mathrm{H}, \mathrm{CH}_{3}-\mathrm{C}=\mathrm{C}-\mathrm{CH}_{3}\right), 1.34\left(\mathrm{~d},{ }^{3} \mathrm{~J}_{\mathrm{HH}}=6.8 \mathrm{~Hz}, 6 \mathrm{H}, \mathrm{CH}\left(\mathrm{CH}_{3}\right)_{2}\right), 1.15\left(\mathrm{~d},{ }^{3} J_{\mathrm{HH}}=6.2 \mathrm{~Hz}, 6 \mathrm{H}\right.$, $\left.\mathrm{CH}\left(\mathrm{CH}_{3}\right)_{2}\right), 1.13\left(\mathrm{~d},{ }^{3} \mathrm{~J}_{\mathrm{HH}}=6.3 \mathrm{~Hz}, 6 \mathrm{H}, \mathrm{CH}\left(\mathrm{CH}_{3}\right)_{2}\right), 1.10\left(\mathrm{~d},{ }^{3} \mathrm{~J}_{\mathrm{HH}}=6.8 \mathrm{~Hz}, 6 \mathrm{H}, \mathrm{CH}\left(\mathrm{CH}_{3}\right)_{2}\right), 0.13(\mathrm{~s}$, 18H, $\left.\mathrm{Si}\left(\mathrm{CH}_{3}\right)_{2}\right),-0.21\left(\mathrm{~s}, 18 \mathrm{H}, \mathrm{Si}\left(\mathrm{CH}_{3}\right)_{2}\right) .{ }^{13} \mathrm{C}$ NMR $\left(101 \mathrm{MHz}, \mathrm{THF}-d_{8}, 23{ }^{\circ} \mathrm{C}\right) \delta 153.25,145.60$, 122.87, $121.03(\mathrm{Ar}-\mathrm{C}), 115.25\left(\mathrm{CH}_{3}-\mathrm{C}=\mathrm{C}-\mathrm{CH}_{3}\right), 25.84\left(\mathrm{CH}\left(\mathrm{CH}_{3}\right)_{2}\right), 25.64\left(\mathrm{CH}_{3}-\mathrm{C}=\mathrm{C}-\mathrm{CH}_{3}\right), 20.32$ $\left(\mathrm{CH}\left(\mathrm{CH}_{3}\right)_{2}\right), 5.83\left(\mathrm{Si}\left(\mathrm{CH}_{3}\right)_{3}\right) .{ }^{29} \mathrm{Si}$ NMR $\left(79 \mathrm{MHz}, \mathrm{THF}-d_{8}, 23{ }^{\circ} \mathrm{C}\right) \delta-15.10 . \mathbf{I R}: \tilde{v}\left(\mathrm{~cm}^{-1}\right) 2958 \mathrm{~m}$, $1416 \mathrm{~m}, 1238 \mathrm{~s}, 981 \mathrm{~s}, 819 \mathrm{~s}, 663 \mathrm{~m}, 591 \mathrm{~m}$.

Synthesis of K[LY(N(SiMe3)2 $\left.)_{2}\right]$ (5): A mixture of LY(THF $)_{2}(\mu-\mathrm{Cl})_{2} \operatorname{Li}(\mathrm{THF})_{2}(1.57 \mathrm{~g}, 2 \mathrm{mmol})$, and $\mathrm{KN}\left(\mathrm{SiMe}_{3}\right)_{2}(0.88 \mathrm{~g}, 4.4 \mathrm{mmol})$ was added $50 \mathrm{~mL} n$-hexane. The solution was stirred at $0{ }^{\circ} \mathrm{C}$ for $8 \mathrm{~h}$. After filtration, the solid was extracted by toluene at $80{ }^{\circ} \mathrm{C}$. The toluene filtrate was concentrated and then kept overnight at $-35{ }^{\circ} \mathrm{C}$ to afford brown solid of $5(1.20 \mathrm{~g}, 65 \%) .{ }^{1} \mathbf{H}$ NMR $\left(400 \mathrm{MHz}, \mathrm{THF}-d_{8}\right.$, $\left.23{ }^{\circ} \mathrm{C}\right) \delta 6.89\left(\mathrm{~d},{ }^{3} J_{\mathrm{HH}}=7.5 \mathrm{~Hz}, 4 \mathrm{H}, \mathrm{Ar}-H\right), 6.73\left(\mathrm{t},{ }^{3} J_{\mathrm{HH}}=7.5 \mathrm{~Hz}, 2 \mathrm{H}, \mathrm{Ar}-H\right), 3.97\left(\mathrm{br}, 4 \mathrm{H}, \mathrm{CH}\left(\mathrm{CH}_{3}\right)_{2}\right)$, $3.62(\mathrm{~m}, 4 \mathrm{H}, \mathrm{THF}-\mathrm{H}), 1.77$ (m, 4H, THF- $H), 1.39$ (s, 6H, $\left.\mathrm{CH}_{3}-\mathrm{C}=\mathrm{C}-\mathrm{CH}_{3}\right), 1.27\left(\mathrm{br}, 12 \mathrm{H}, \mathrm{CH}\left(\mathrm{CH}_{3}\right)_{2}\right)$, $1.12\left(\mathrm{~d},{ }^{3} J_{\mathrm{HH}}=6.7 \mathrm{~Hz}, 12 \mathrm{H}, \mathrm{CH}\left(\mathrm{CH}_{3}\right)_{2}\right), 0.00\left(\mathrm{br}, 36 \mathrm{H}, \mathrm{Si}\left(\mathrm{CH}_{3}\right)_{2}\right) .{ }^{13} \mathbf{C}$ NMR $\left(101 \mathrm{MHz}, \mathrm{THF}-d_{8}, 23\right.$ $\left.{ }^{\circ} \mathrm{C}\right) \delta 153.10,146.04,122.82,121.18(\mathrm{Ar}-\mathrm{C}), 116.81\left(\mathrm{CH}_{3}-\mathrm{C}=\mathrm{C}-\mathrm{CH}_{3}\right), 68.03,25.46$ (THF-C), 27.30 $\left(\mathrm{CH}_{3}-\mathrm{C}=\mathrm{C}-\mathrm{CH}_{3}\right), 26.19\left(\mathrm{CH}\left(\mathrm{CH}_{3}\right)_{2}\right), 20.00\left(\mathrm{CH}\left(\mathrm{CH}_{3}\right)_{2}\right), 6.41\left(\mathrm{Si}\left(\mathrm{CH}_{3}\right)_{3}\right) .{ }^{29} \mathrm{Si}$ NMR $(79 \mathrm{MHz}, \mathrm{THF}-$ $\left.d_{8}, 23{ }^{\circ} \mathrm{C}\right) \delta-5.94 . \mathbf{I R}: \tilde{v}\left(\mathrm{~cm}^{-1}\right) 2952 \mathrm{~m}, 1418 \mathrm{~m}, 1237 \mathrm{~s}, 945 \mathrm{~s}, 825 \mathrm{~s}, 665 \mathrm{~m}, 603 \mathrm{~m}$. 
Typical Procedure for Synthesis of 1,3-Enyne. A Schlenk bottle was charged with $\mathrm{Pd}\left(\mathrm{PPh}_{3}\right)_{4}(0.25$ mmol, $2.5 \mathrm{~mol} \%), \mathrm{CuI}(0.5 \mathrm{mmol}, 5 \mathrm{~mol} \%)$ and diisopropylamine $(30 \mathrm{~mL})$. And then terminal alkyne (10 mmol) and vinyl bromide $(10 \mathrm{mmol})$ were added in sequence. After stirring at $70{ }^{\circ} \mathrm{C}$ in $\mathrm{Ar}$ atmosphere for $12 \mathrm{~h}$, the mixture was concentrated under vacuum condition, and then purified by vacuum distillation in Ar atmosphere or flash column chromatography ( $n$-hexane) in an Ar glove box to give the desired product 7 .

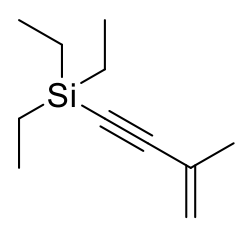

7d

Colorless oil 7d (yield $85 \%$ ). ${ }^{1} \mathbf{H}$ NMR $\left(400 \mathrm{MHz}, \mathrm{CDCl}_{3}, 23{ }^{\circ} \mathrm{C}\right) \delta 5.34\left(\mathrm{~s}, 1 \mathrm{H}, \mathrm{C}=\mathrm{CH}_{2}\right)$, $5.24\left(\mathrm{~s}, 1 \mathrm{H}, \mathrm{C}=\mathrm{C} H_{2}\right), 1.90(\mathrm{~s}, 3 \mathrm{H}, \mathrm{C}=\mathrm{C}(\mathrm{CH})), 1.00\left(\mathrm{t},{ }^{3} \mathrm{~J}_{\mathrm{HH}}=7.9 \mathrm{~Hz}, 9 \mathrm{H}, \mathrm{Si}\left(\mathrm{CH}_{2} \mathrm{CH}_{3}\right)_{3}\right), 0.62\left(\mathrm{q},{ }^{3} J_{\mathrm{HH}}\right.$ $\left.=7.9 \mathrm{~Hz}, 6 \mathrm{H}, \mathrm{Si}\left(\mathrm{CH}_{2} \mathrm{CH}_{3}\right)_{3}\right) .{ }^{13} \mathbf{C} \mathbf{N M R}\left(101 \mathrm{MHz}, \mathrm{CDCl}_{3}, 23{ }^{\circ} \mathrm{C}\right) \delta 127.15,122.70\left(\mathrm{C}=\mathrm{C}\left(\mathrm{CH}_{3}\right)\right)$, 107.84, $90.49\left(\mathrm{CCSi}\left(\mathrm{CH}_{2} \mathrm{CH}_{3}\right)_{3}\right), 23.60\left(\mathrm{C}=\mathrm{C}\left(\mathrm{CH}_{3}\right)\right), 7.58,4.55\left(\mathrm{Si}\left(\mathrm{CH}_{2} \mathrm{CH}_{3}\right)_{3}\right) .{ }^{29} \mathrm{Si}$ NMR $(79 \mathrm{MHz}$, $\left.\mathrm{CDCl}_{3}, 23{ }^{\circ} \mathrm{C}\right) \delta-7.57\left(\mathrm{Si}\left(\mathrm{CH}_{2} \mathrm{CH}_{3}\right)_{3}\right)$. EI-MS for $\mathrm{M}^{+}: \mathrm{m} / \mathrm{z} 180.07$.

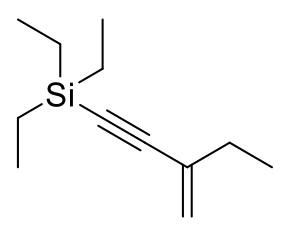

$7 e$

Colorless oil 7e (yield 89 \%). ${ }^{1} \mathbf{H}$ NMR $\left(400 \mathrm{MHz}, \mathrm{CDCl}_{3}, 23{ }^{\circ} \mathrm{C}\right) \delta 5.35(\mathrm{~s}, 1 \mathrm{H}$, $\left.\mathrm{C}=\mathrm{CH}_{2}\right), 5.24\left(\mathrm{~s}, 1 \mathrm{H}, \mathrm{C}=\mathrm{CH} \mathrm{CH}_{2}\right), 2.18\left(\mathrm{q},{ }^{3} \mathrm{~J}_{\mathrm{HH}}=7.4 \mathrm{~Hz}, 2 \mathrm{H}, \mathrm{C}=\mathrm{C}\left(\mathrm{CH}_{2} \mathrm{CH}_{3}\right)\right), 1.11\left(\mathrm{t},{ }^{3} \mathrm{~J}_{\mathrm{HH}}=7.5 \mathrm{~Hz}, 3 \mathrm{H}\right.$, $\left.\mathrm{C}=\mathrm{C}\left(\mathrm{CH}_{2} \mathrm{CH}_{3}\right)\right), 1.01\left(\mathrm{t},{ }^{3} \mathrm{~J}_{\mathrm{HH}}=7.9 \mathrm{~Hz}, 9 \mathrm{H}, \mathrm{Si}\left(\mathrm{CH}_{2} \mathrm{CH}_{3}\right)_{3}\right), 0.62\left(\mathrm{q},{ }^{3} J_{\mathrm{HH}}=7.9 \mathrm{~Hz}, 6 \mathrm{H}, \mathrm{Si}\left(\mathrm{CH}_{2} \mathrm{CH}_{3}\right)_{3}\right)$. ${ }^{13} \mathrm{C}$ NMR $\left(101 \mathrm{MHz}, \mathrm{CDCl}_{3}, 23{ }^{\circ} \mathrm{C}\right) \quad \delta$ 133.53, $120.78 \quad\left(C=C\left(\mathrm{CH}_{2} \mathrm{CH}_{3}\right)\right), \quad 107.03,91.32$ $\left(C C \mathrm{Si}\left(\mathrm{CH}_{2} \mathrm{CH}_{3}\right)_{3}\right), 30.50\left(\mathrm{C}=\mathrm{C}\left(\mathrm{CH}_{2} \mathrm{CH}_{3}\right)\right), 12.91\left(\mathrm{C}=\mathrm{C}\left(\mathrm{CH}_{2} \mathrm{CH}_{3}\right)\right), 7.61,4.57\left(\mathrm{SiEt}_{3}-\mathrm{C}\right) . \mathbf{E I}-\mathbf{M S}$ for $\mathrm{M}^{+}: \mathrm{m} / \mathrm{z} 194.15$.

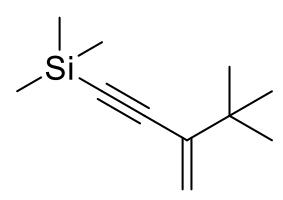

Colorless oil 7f (yield $82 \%) .{ }^{1} \mathbf{H}$ NMR (400 MHz, $\left.\mathrm{CDCl}_{3}, 23{ }^{\circ} \mathrm{C}\right) \delta 5.35(\mathrm{~s}, 1 \mathrm{H}$, 
$\left.\mathrm{C}=\mathrm{CH}_{2}\right), 5.27\left(\mathrm{~s}, 1 \mathrm{H}, \mathrm{C}=\mathrm{CH}_{2}\right), 1.13\left(\mathrm{~s}, 9 \mathrm{H}, \mathrm{C}\left(\mathrm{CH}_{3}\right)_{3}\right), 0.19\left(\mathrm{~s}, 9 \mathrm{H}, \mathrm{Si}\left(\mathrm{CH}_{3}\right)_{3}\right) .{ }^{13} \mathrm{C}$ NMR $(101 \mathrm{MHz}$, $\left.\mathrm{CDCl}_{3}, 23{ }^{\circ} \mathrm{C}\right) \delta 141.87,118.60\left(C=C\left(\mathrm{CMe}_{3}\right)\right), 105.39,94.79\left(\mathrm{Si}\left(\mathrm{CH}_{3}\right)_{3} C C\right), 35.94\left(C\left(\mathrm{CH}_{3}\right)_{3}\right), 29.06$ $\left(\mathrm{C}\left(\mathrm{CH}_{3}\right)_{3}\right), 0.16\left(\mathrm{Si}\left(\mathrm{CH}_{3}\right)_{3}\right) . \mathbf{E I}-\mathbf{M S}$ for $\mathrm{M}^{+}: \mathrm{m} / \mathrm{z} 180.18$.

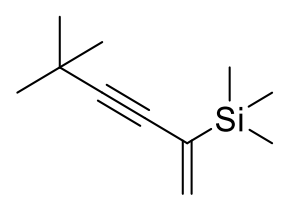

7g Colorless oil $7 \mathbf{g}$ (yield $83 \%) .{ }^{\mathbf{1}} \mathbf{H}$ NMR $\left(400 \mathrm{MHz}, \mathrm{CDCl}_{3}, 23{ }^{\circ} \mathrm{C}\right) \delta 5.96\left(\mathrm{~d},{ }^{2} \mathrm{~J}_{\mathrm{HH}}=\right.$ $\left.3.4 \mathrm{~Hz}, 1 \mathrm{H}, \mathrm{C}=\mathrm{CH}_{2}\right), 5.57\left(\mathrm{~d},{ }^{2} \mathrm{~J}_{\mathrm{HH}}=3.4 \mathrm{~Hz}, 1 \mathrm{H}, \mathrm{C}=\mathrm{C} H_{2}\right), 1.25\left(\mathrm{~s}, 9 \mathrm{H}, \mathrm{C}\left(\mathrm{CH}_{3}\right)_{3}\right), 0.14\left(\mathrm{~s}, 9 \mathrm{H}, \mathrm{Si}(\mathrm{CH})_{3}\right)$. ${ }^{13} \mathrm{C}$ NMR $\left(101 \mathrm{MHz}, \mathrm{CDCl}_{3}, 23{ }^{\circ} \mathrm{C}\right) \delta 135.33,131.94\left(C=C\left(\mathrm{Si}\left(\mathrm{CH}_{3}\right)_{3}\right)\right), 103.70,80.16\left(\mathrm{CMe}_{3} C C\right)$, $31.34\left(\mathrm{C}\left(\mathrm{CH}_{3}\right)_{3}\right), 28.31\left(\mathrm{C}\left(\mathrm{CH}_{3}\right)_{3}\right),-2.06\left(\mathrm{Si}\left(\mathrm{CH}_{3}\right)_{3}\right) . \mathbf{E I}-\mathbf{M S}$ for $\mathrm{M}^{+}: \mathrm{m} / \mathrm{z} 180.10$.

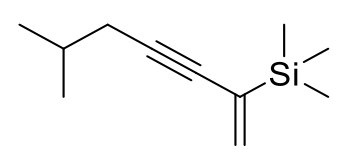

7h Colorless oil $7 \mathbf{h}$ (yield $87 \%$ ). ${ }^{\mathbf{1}} \mathbf{H}$ NMR $\left(400 \mathrm{MHz}, \mathrm{CDCl}_{3}, 23{ }^{\circ} \mathrm{C}\right) \delta 6.00\left(\mathrm{~d},{ }^{2} \mathrm{~J}_{\mathrm{HH}}\right.$ $\left.=3.5 \mathrm{~Hz}, 1 \mathrm{H}, \mathrm{C}=\mathrm{CH} H_{2}\right), 5.60\left(\mathrm{~d},{ }^{2} J_{\mathrm{HH}}=3.5 \mathrm{~Hz}, 1 \mathrm{H}, \mathrm{C}=\mathrm{CH}_{2}\right), 2.26\left(\mathrm{~d},{ }^{3} J_{\mathrm{HH}}=6.5 \mathrm{~Hz}, 2 \mathrm{H}, i \operatorname{PrCH}\right), 1.83$ (hept, $\left.{ }^{3} J_{\mathrm{HH}}=6.6 \mathrm{~Hz}, 1 \mathrm{H}, i \mathrm{Pr}-\mathrm{CH}\right), 0.99\left(\mathrm{~d},{ }^{3} J_{\mathrm{HH}}=6.7 \mathrm{~Hz}, 6 \mathrm{H}, i \mathrm{Pr}-\mathrm{CH} H_{3}\right), 0.15\left(\mathrm{~s}, 9 \mathrm{H}, \mathrm{Si}\left(\mathrm{CH}_{3}\right)_{3}\right) .{ }^{13} \mathbf{C}$ NMR $\left(101 \mathrm{MHz} \mathrm{CDCl}_{3}, 23{ }^{\circ} \mathrm{C}\right) \delta 135.43,132.58\left(C=C\left(\mathrm{Si}\left(\mathrm{CH}_{3}\right)_{3}\right)\right), 93.69,82.72\left({ }_{\mathrm{PrCH}} \mathrm{CC}\right), 29.02$, 28.50, $22.15\left(i \mathrm{PrCH}_{2}-\mathrm{C}\right),-1.98\left(\mathrm{Si}\left(\mathrm{CH}_{3}\right)_{3}\right)$. EI-MS for $\mathrm{M}^{+}: \mathrm{m} / \mathrm{z} 180.14$.

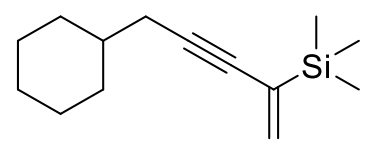

$7 \mathbf{i}$

Pale yellow oil 7i (yield $81 \%$ ). ${ }^{\mathbf{1}} \mathbf{H}$ NMR $\left(400 \mathrm{MHz}, \mathrm{CDCl}_{3}, 23{ }^{\circ} \mathrm{C}\right) \delta 5.99(\mathrm{~d}$, $\left.{ }^{2} J_{\mathrm{HH}}=3.6 \mathrm{~Hz}, 1 \mathrm{H}, \mathrm{C}=\mathrm{CH}_{2}\right), 5.60\left(\mathrm{~d},{ }^{2} J_{\mathrm{HH}}=3.5 \mathrm{~Hz}, 1 \mathrm{H}, \mathrm{C}=\mathrm{CH}_{2}\right), 2.25\left(\mathrm{~d},{ }^{3} J_{\mathrm{HH}}=6.5 \mathrm{~Hz}, 2 \mathrm{H}, \mathrm{CyCH}\right)_{2}$, 1.81 (m, 2H, Cy-CH2), 1.73 (m, 2H, Cy-CH2), 1.66 (m, 1H, Cy-CH2), 1.48 (m, 1H, Cy-CH), 1.29-0.97 (m, 5H, Cy-CH2), $0.15\left(\mathrm{~s}, 9 \mathrm{H}, \mathrm{Si}\left(\mathrm{CH}_{3}\right)_{3}\right) .{ }^{13} \mathrm{C}$ NMR $\left(101 \mathrm{MHz}, \mathrm{CDCl}_{3}, 23{ }^{\circ} \mathrm{C}\right) \delta 135.45,132.47$ $\left(C=C\left(\mathrm{Si}\left(\mathrm{CH}_{3}\right)_{3}\right)\right), 93.72,82.70\left(\mathrm{CyCH}_{2} \mathrm{CC}\right), 37.85,32.88,29.86,27.64,26.49,26.33\left(\mathrm{CyCH}_{2}-C\right)$, $-1.97\left(\mathrm{Si}\left(\mathrm{CH}_{3}\right)_{3}\right) . \mathbf{E I}-\mathbf{M S}$ for $\mathrm{M}^{+}: \mathrm{m} / \mathrm{z} 220.09$. 


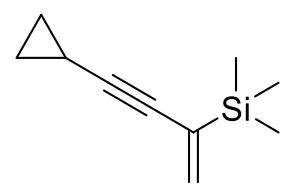

7j Colorless oil 7j (yield 82\%). ${ }^{\mathbf{1}} \mathbf{H} \mathbf{N M R}\left(400 \mathrm{MHz}, \mathrm{CDCl}_{3}, 23{ }^{\circ} \mathrm{C}\right) \delta 5.98\left(\mathrm{~d},{ }^{2} \mathrm{~J}_{\mathrm{HH}}=3.4\right.$ $\left.\mathrm{Hz}, 1 \mathrm{H}, \mathrm{C}=\mathrm{CH}_{2}\right), 5.58\left(\mathrm{~d},{ }^{2} \mathrm{~J}_{\mathrm{HH}}=3.4 \mathrm{~Hz}, 1 \mathrm{H}, \mathrm{C}=\mathrm{CH}_{2}\right), 1.38\left(\mathrm{~m}, 1 \mathrm{H}, c \mathrm{C}_{3} \mathrm{H}_{5}-\mathrm{CH}\right), 0.82\left(\mathrm{~m}, 2 \mathrm{H}, c \mathrm{C}_{3} \mathrm{H}_{5}-\right.$ $\left.\mathrm{CH}_{2}\right), 0.70\left(\mathrm{~m}, 2 \mathrm{H}, c \mathrm{C}_{3} \mathrm{H}_{5}-\mathrm{CH}_{2}\right), 0.13\left(\mathrm{~s}, 9 \mathrm{H}, \mathrm{Si}\left(\mathrm{CH}_{3}\right)_{3}\right) .{ }^{13} \mathbf{C ~ N M R}\left(101 \mathrm{MHz}, \mathrm{CDCl}_{3}, 23{ }^{\circ} \mathrm{C}\right) \delta 135.22$, $132.50\left(C=C\left(\mathrm{SiMe}_{3}\right)\right), 98.31,82.68\left(c \mathrm{C}_{3} \mathrm{H}_{5} C C\right), 9.00,0.57\left(c \mathrm{C}_{3} \mathrm{H}_{5}-C\right),-2.00\left(\mathrm{Si}\left(C_{3}\right)_{3}\right) . \mathbf{E I}-\mathbf{M S}$ for $\mathrm{M}^{+}: \mathrm{m} / \mathrm{z} 164.02$.

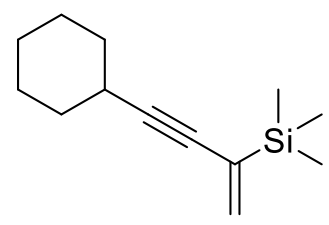

7k Colorless oil 7k (yield $76 \%$ ). ${ }^{\mathbf{1}} \mathbf{H}$ NMR (400 MHz, $\left.\mathrm{CDCl}_{3}, 23{ }^{\circ} \mathrm{C}\right) \delta 5.98\left(\mathrm{~d},{ }^{2} J_{\mathrm{HH}}\right.$ $\left.=3.2 \mathrm{~Hz}, 1 \mathrm{H}, \mathrm{C}=\mathrm{C} H_{2}\right), 5.59\left(\mathrm{~d},{ }^{2} \mathrm{~J}_{\mathrm{HH}}=3.3 \mathrm{~Hz}, 1 \mathrm{H}, \mathrm{C}=\mathrm{CH} H_{2}\right), 2.53(\mathrm{~m}, 1 \mathrm{H}, \mathrm{Cy}-\mathrm{CH}), 1.80(\mathrm{~m}, 2 \mathrm{H}, \mathrm{Cy}-$ $\left.\mathrm{CH}_{2}\right), 1.71\left(\mathrm{~m}, 2 \mathrm{H}, \mathrm{Cy}-\mathrm{CH}_{2}\right), 1.52-1.25\left(\mathrm{~m}, 6 \mathrm{H}, \mathrm{Cy}-\mathrm{CH}_{2}\right), 0.15\left(\mathrm{~s}, 9 \mathrm{H}, \mathrm{Si}\left(\mathrm{CH}_{3}\right)_{3}\right) .{ }^{13} \mathrm{C}$ NMR $(101 \mathrm{MHz}$, $\left.\mathrm{CDCl}_{3}, 23{ }^{\circ} \mathrm{C}\right) \delta 135.41,132.18\left(C=C\left(\mathrm{Si}\left(\mathrm{CH}_{3}\right)_{3}\right)\right), 99.28,81.70(\mathrm{CyCC}), 33.08,30.04,29.86,26.13$, $25.01(\mathrm{Cy}-\mathrm{C}),-2.01\left(\mathrm{Si}\left(\mathrm{CH}_{3}\right)_{3}\right)$. EI-MS for $\mathrm{M}^{+}: \mathrm{m} / \mathrm{z} 206.17$.

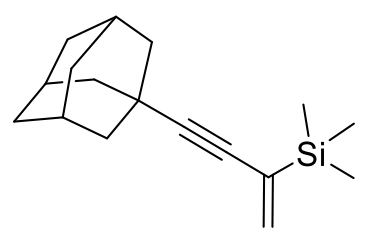

$71 \quad$ Pale yellow oil 71 (yield $86 \%$ ). ${ }^{1} \mathbf{H}$ NMR $\left(400 \mathrm{MHz}, \mathrm{CDCl}_{3}, 23{ }^{\circ} \mathrm{C}\right) \delta 5.96(\mathrm{~d}$, $\left.{ }^{2} J_{\mathrm{HH}}=2.6 \mathrm{~Hz}, 1 \mathrm{H}, \mathrm{C}=\mathrm{CH}_{2}\right), 5.57\left(\mathrm{~d},{ }^{2} J_{\mathrm{HH}}=2.6 \mathrm{~Hz}, 1 \mathrm{H}, \mathrm{C}=\mathrm{CH}\right), 1.96(\mathrm{br}, 3 \mathrm{H}$, adamantane-CH$), 1.89$ (br, 6H, adamantane- $\left.\mathrm{CH}_{2}\right), 1.69\left(\mathrm{br}, 6 \mathrm{H}\right.$, adamantane- $\left.\mathrm{CH}_{2}\right), 0.14\left(\mathrm{~s}, 9 \mathrm{H}, \mathrm{Si}\left(\mathrm{CH}_{3}\right)_{3}\right) .{ }^{\mathbf{1 3}} \mathbf{C} \mathbf{~ N M R}(101$ $\left.\mathrm{MHz}, \mathrm{CDCl}_{3}, 23{ }^{\circ} \mathrm{C}\right) \delta 135.31,131.85\left(C=C\left(\mathrm{Si}\left(\mathrm{CH}_{3}\right)_{3}\right)\right), 103.70,80.42$ (adamantane $\left.C C\right), 43.25,42.51$, 36.58, 36.37, 30.45, 28.24, 27.94 (adamantane-C), -2.06 $\left(\mathrm{Si}\left(\mathrm{CH}_{3}\right)_{3}\right) . \mathbf{E I}-\mathbf{M S}$ for $\mathrm{M}^{+}: \mathrm{m} / \mathrm{z} 258.11$.

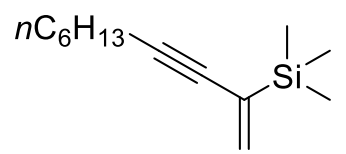

$7 \mathbf{m} \quad$ Colorless oil $\mathbf{7 m}$ (yield 89\%). ${ }^{\mathbf{1}} \mathbf{H} \mathbf{N M R}\left(400 \mathrm{MHz}, \mathrm{CDCl}_{3}, 23{ }^{\circ} \mathrm{C}\right) \delta 5.99\left(\mathrm{~d},{ }^{2} J_{\mathrm{HH}}\right.$ $\left.=3.5 \mathrm{~Hz}, 1 \mathrm{H}, \mathrm{C}=\mathrm{C} H_{2}\right), 5.59\left(\mathrm{~d},{ }^{2} J_{\mathrm{HH}}=3.5 \mathrm{~Hz}, 1 \mathrm{H}, \mathrm{C}=\mathrm{C} H_{2}\right), 2.35\left(\mathrm{t},{ }^{3} J_{\mathrm{HH}}=7.0 \mathrm{~Hz}, 2 \mathrm{H}, \mathrm{CCCH}\right), 1.53$ 
$\left(\mathrm{m}, 2 \mathrm{H}, n \mathrm{Hex}-\mathrm{CH}_{2}\right), 1.41\left(\mathrm{~m}, 2 \mathrm{H}, n \mathrm{Hex}-\mathrm{CH}_{2}\right), 1.30\left(\mathrm{~m}, 4 \mathrm{H}, n \mathrm{Hex}-\mathrm{CH}_{2}\right), 0.89\left(\mathrm{t},{ }^{3} J_{\mathrm{HH}}=6.9 \mathrm{~Hz}, 3 \mathrm{H}\right.$, $\left.n \mathrm{Hex}-\mathrm{CH}_{3}\right), 0.14\left(\mathrm{~s}, 9 \mathrm{H}, \mathrm{Si}\left(\mathrm{CH}_{3}\right)_{3}\right) .{ }^{13} \mathbf{C} \mathbf{N M R}\left(101 \mathrm{MHz}, \mathrm{CDCl}_{3}, 23{ }^{\circ} \mathrm{C}\right) \delta 135.43,132.50$ $\left(C=C\left(\mathrm{Si}\left(\mathrm{CH}_{3}\right)_{3}\right)\right), 94.94,81.82(n \mathrm{Hex} C C), 31.53,29.14,28.71,22.75,19.79,14.21(n \mathrm{Hex}-C),-2.01$ $\left(\mathrm{Si}\left(\mathrm{CH}_{3}\right)_{3}\right)$. EI-MS for $\mathrm{M}^{+}: \mathrm{m} / \mathrm{z} 208.10$.

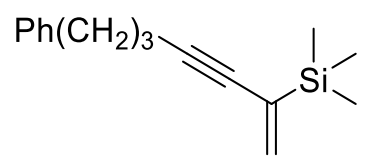

$7 n$

Pale yellow oil 7n (yield 83 \%). ${ }^{\mathbf{1}} \mathbf{H}$ NMR $\left(400 \mathrm{MHz}, \mathrm{CDCl}_{3}, 23{ }^{\circ} \mathrm{C}\right) \delta 7.29(\mathrm{~m}$, $2 \mathrm{H}, \operatorname{Ar}-H), 7.20(\mathrm{~m}, 3 \mathrm{H}, \mathrm{Ar}-H), 6.04\left(\mathrm{~d},{ }^{2} \mathrm{~J}_{\mathrm{HH}}=3.5 \mathrm{~Hz}, 1 \mathrm{H}, \mathrm{C}=\mathrm{CH}_{2}\right), 5.64\left(\mathrm{~d},{ }^{2} J_{\mathrm{HH}}=3.5 \mathrm{~Hz}, 1 \mathrm{H}\right.$, $\left.\mathrm{C}=\mathrm{CH}_{2}\right), 2.76\left(\mathrm{t},{ }^{3} \mathrm{~J}_{\mathrm{HH}}=7.6 \mathrm{~Hz}, 2 \mathrm{H}, \mathrm{PhCH}_{2} \mathrm{CH}_{2} \mathrm{CH}_{2}\right), 2.39\left(\mathrm{t},{ }^{3} \mathrm{~J}_{\mathrm{HH}}=6.9 \mathrm{~Hz}, 2 \mathrm{H}, \mathrm{PhCH}_{2} \mathrm{CH}_{2} \mathrm{CH}_{2}\right), 1.87$ (quint, $\left.{ }^{3} J_{\mathrm{HH}}=7.6 \mathrm{~Hz}, 2 \mathrm{H}, \mathrm{PhCH}_{2} \mathrm{CH}_{2} \mathrm{CH}_{2}\right), 0.18\left(\mathrm{~s}, 9 \mathrm{H}, \mathrm{Si}\left(\mathrm{CH}_{3}\right)_{3}\right) .{ }^{13} \mathbf{C ~ N M R}\left(101 \mathrm{MHz}, \mathrm{CDCl}_{3}, 23\right.$ $\left.{ }^{\circ} \mathrm{C}\right) \delta 141.94,135.31,132.83,128.69,128.49,126.00\left(\mathrm{Ar}-\mathrm{C}+C=C\left(\mathrm{Si}\left(\mathrm{CH}_{3}\right)_{3}\right)\right), 94.18,82.41$ $\left(\mathrm{PhCH}_{2} \mathrm{CH}_{2} \mathrm{CH}_{2} \mathrm{CC}\right), 35.03,30.87,19.29\left(\mathrm{PhCH}_{2} \mathrm{CH}_{2} \mathrm{CH}_{2}\right),-1.95\left(\mathrm{Si}\left(\mathrm{CH}_{3}\right)_{3}\right) . \mathbf{E I}-\mathbf{M S}$ for $\mathrm{M}^{+}: \mathrm{m} / \mathrm{z}$ 242.13.

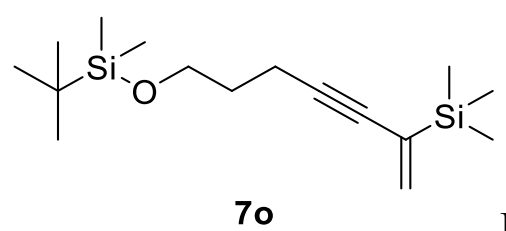

Pale yellow oil $7 \mathbf{0}$ (yield $76 \%$ ). ${ }^{1} \mathbf{H}$ NMR $\left(400 \mathrm{MHz}, \mathrm{CDCl}_{3}, 23{ }^{\circ} \mathrm{C}\right) \delta$ $6.24\left(\mathrm{~d},{ }^{2} J_{\mathrm{HH}}=3.6 \mathrm{~Hz}, 1 \mathrm{H}, \mathrm{C}=\mathrm{CH}_{2}\right), 5.67\left(\mathrm{~d},{ }^{2} J_{\mathrm{HH}}=3.5 \mathrm{~Hz}, 1 \mathrm{H}, \mathrm{C}=\mathrm{C} H_{2}\right), 3.70\left(\mathrm{t},{ }^{3} J_{\mathrm{HH}}=6.0 \mathrm{~Hz}, 2 \mathrm{H}\right.$, $\mathrm{SiOCH}_{2}$ ), $2.49\left(\mathrm{t},{ }^{3} \mathrm{~J}_{\mathrm{HH}}=7.0 \mathrm{~Hz}, 2 \mathrm{H}, \mathrm{SiOCH}_{2} \mathrm{CH}_{2} \mathrm{CH}_{2}\right.$ ), 1.75 (quint, ${ }^{3} J_{\mathrm{HH}}=6.5 \mathrm{~Hz}, 2 \mathrm{H}$, $\left.\mathrm{SiOCH}_{2} \mathrm{CH}_{2} \mathrm{CH}_{2}\right), 1.05$ (s, 9H, C(CH3) $\left.)_{3}\right), 0.28\left(\mathrm{~s}, 9 \mathrm{H}, \mathrm{Si}\left(\mathrm{CH}_{3}\right)_{3}\right), 0.15$ (s, 6H, $\left.\mathrm{Si}\left(\mathrm{CH}_{3}\right)_{2}\right) .{ }^{13} \mathbf{C}$ NMR $\left(101 \mathrm{MHz}, \mathrm{CDCl}_{3}, 23{ }^{\circ} \mathrm{C}\right) \delta 135.33,132.71\left(\mathrm{C}=\mathrm{C}\left(\mathrm{Si}\left(\mathrm{CH}_{3}\right)_{3}\right)\right), 94.19,81.94\left(\mathrm{SiOCH}_{2} \mathrm{CH}_{2} \mathrm{CH}_{2} C C\right)$, 61.86, 32.25, 26.10, 18.50, $16.21\left(C\left(\mathrm{CH}_{3}\right)_{3}+\mathrm{SiOCH}_{2} \mathrm{CH}_{2} \mathrm{CH}_{2}\right),-1.99\left(\mathrm{Si}\left(\mathrm{CH}_{3}\right)_{3}\right),-5.16\left(\mathrm{OSi}_{(}\left(\mathrm{CH}_{3}\right)_{2}\right)$.

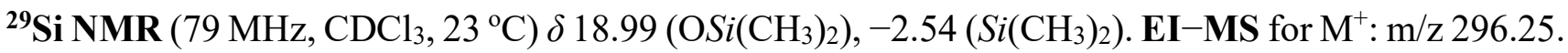

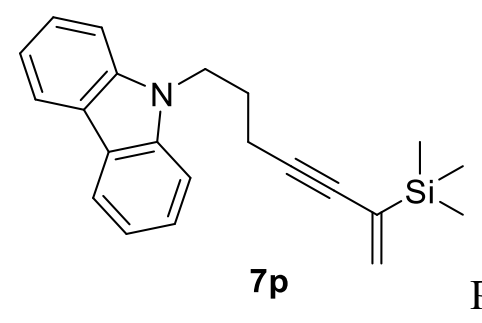

Pale yellow oil 7p (yield $84 \%$ ). ${ }^{\mathbf{1}} \mathbf{H}$ NMR $\left(400 \mathrm{MHz}, \mathrm{CDCl}_{3}, 23{ }^{\circ} \mathrm{C}\right) \delta 7.89$ 
$\left(\mathrm{d},{ }^{3} \mathrm{~J}_{\mathrm{HH}}=7.8 \mathrm{~Hz}, 2 \mathrm{H}, \operatorname{Ar}-H\right), 7.26(\mathrm{~m}, 4 \mathrm{H}, \operatorname{Ar}-H), 7.01(\mathrm{~m}, 2 \mathrm{H}, \operatorname{Ar}-H), 5.91\left(\mathrm{~d},{ }^{2} J_{\mathrm{HH}}=3.5 \mathrm{~Hz}, 1 \mathrm{H}\right.$, $\left.\mathrm{C}=\mathrm{CH}_{2}\right), 5.50\left(\mathrm{~d},{ }^{2} J_{\mathrm{HH}}=3.5 \mathrm{~Hz}, 1 \mathrm{H}, \mathrm{C}=\mathrm{CH}_{2}\right), 4.24\left(\mathrm{t},{ }^{3} J_{\mathrm{HH}}=6.8 \mathrm{~Hz}, 2 \mathrm{H}, \mathrm{NCH}\right), 2.20\left(\mathrm{t},{ }^{3} J_{\mathrm{HH}}=6.6\right.$ $\mathrm{Hz}, 2 \mathrm{H}, \mathrm{NCH}_{2} \mathrm{CH}_{2} \mathrm{CH}_{2}$ ), 1.89 (quint, $\left.{ }^{3} \mathrm{~J}_{\mathrm{HH}}=6.7 \mathrm{~Hz}, 2 \mathrm{H}, \mathrm{NCH}_{2} \mathrm{CH}_{2} \mathrm{CH}_{2}\right), 0.01\left(\mathrm{~s}, 9 \mathrm{H}, \mathrm{Si}\left(\mathrm{CH}_{3}\right)_{3}\right) .{ }^{13} \mathrm{C}$ NMR $\left(101 \mathrm{MHz}, \mathrm{CDCl}_{3}, 23{ }^{\circ} \mathrm{C}\right) \delta 140.59,135.05,133.61,125.80,122.98,120.47,119.01,108.81$ $\left(\mathrm{Ar}-\mathrm{C}+\mathrm{C}=\mathrm{C}\left(\mathrm{Si}\left(\mathrm{CH}_{3}\right)_{3}\right)\right), 93.00,83.03\left(\mathrm{NCH}_{2} \mathrm{CH}_{2} \mathrm{CH}_{2} \mathrm{CC}\right), 41.81,28.41,17.61\left(\mathrm{NCH}_{2} \mathrm{CH}_{2} \mathrm{CH}_{2}\right)$, $-1.89\left(\mathrm{Si}\left(\mathrm{CH}_{3}\right)_{3}\right)$. HRMS (EI) calcd for $\left\{[\mathrm{M}]^{+},\left[\mathrm{C}_{22} \mathrm{H}_{25} \mathrm{NSi}\right]^{+}\right\}: 331.1751$; found 331.1750 . 
Typical Procedure for Catalytic Hydrosilylation. In an Ar glove box, a Schlenk tube was charged with catalyst $2(0.02 \mathrm{mmol}, 5 \mathrm{~mol} \%)$ and toluene $(1 \mathrm{~mL})$. And then hydrosilane $6(0.44 \mathrm{mmol})$ and 1,3-enyne 7 (0.4 mmol) were added in sequence. Conversion and selectivity were analyzed by ${ }^{1} \mathrm{H}$ NMR and GC-MS of the crude reaction mixture after quenching and diluting the crude reaction mixture with $n$-hexane. After stirring at room temperature in Ar atmosphere (6-8 h except as otherwise specified), the mixture was purified by flash column chromatography ( $n$-hexane) to give the desired product.

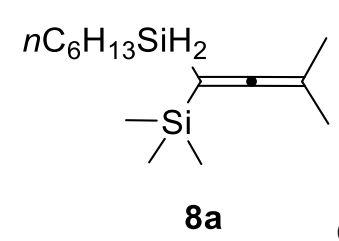

8a Colorless oil 8a (yield $85 \%) .{ }^{\mathbf{1}} \mathbf{H}$ NMR $\left(400 \mathrm{MHz}, \mathrm{CDCl}_{3}, 23{ }^{\circ} \mathrm{C}\right) \delta 3.95\left(\mathrm{t},{ }^{3} J_{\mathrm{HH}}=\right.$ $\left.3.2 \mathrm{~Hz}, 2 \mathrm{H}, \mathrm{Si} H_{2}\right), 1.63\left(\mathrm{~s}, 6 \mathrm{H}, \mathrm{C}=\mathrm{C}\left(\mathrm{CH}_{3}\right)_{2}\right), 1.45-1.24\left(\mathrm{~m}, 8 \mathrm{H}, n \mathrm{Hex}-\mathrm{CH}_{2}\right), 0.89\left(\mathrm{t},{ }^{3} \mathrm{~J}_{\mathrm{HH}}=6.5 \mathrm{~Hz}, 3 \mathrm{H}\right.$, $\left.n \mathrm{Hex}-\mathrm{CH}_{3}\right), 0.74\left(\mathrm{~m}, 2 \mathrm{H}, \mathrm{SiH}_{2} \mathrm{CH}_{2}\right), 0.09\left(\mathrm{~s}, 9 \mathrm{H}, \mathrm{Si}\left(\mathrm{CH}_{3}\right)_{3}\right) .{ }^{13} \mathbf{C} \mathbf{N M R}\left(101 \mathrm{MHz}, \mathrm{CDCl}_{3}, 23{ }^{\circ} \mathrm{C}\right) \delta$ $213.15(\mathrm{C}=C=\mathrm{C}), 80.43,80.10(C=\mathrm{C}=C), 32.72,31.78,25.14,22.76\left(n \mathrm{Hex}-C \mathrm{H}_{2}\right), 18.92\left(\mathrm{C}=\mathrm{C}\left(C \mathrm{H}_{3}\right)_{2}\right)$, $14.28\left(n \mathrm{Hex}-\mathrm{CH}_{3}\right), 10.53\left(\mathrm{SiH}_{2} \mathrm{CH}_{2}\right),-0.45\left(\mathrm{Si}\left(\mathrm{CH}_{3}\right)_{3}\right)$. EI-MS for $\mathrm{M}^{+}: \mathrm{m} / \mathrm{z} 254.17$.

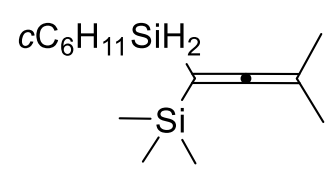

8b Colorless oil $8 \mathbf{b}\left(60{ }^{\circ} \mathrm{C}, 16 \mathrm{~h}\right.$, yield $\left.88 \%\right) .{ }^{1} \mathbf{H} \mathbf{~ N M R}\left(400 \mathrm{MHz}, \mathrm{CDCl}_{3}, 23{ }^{\circ} \mathrm{C}\right) \delta$ $3.82\left(\mathrm{~d},{ }^{3} J_{\mathrm{HH}}=2.9 \mathrm{~Hz}, 2 \mathrm{H}, \mathrm{Si} H_{2}\right), 1.72\left(\mathrm{~m}, 5 \mathrm{H}, \mathrm{Cy}-\mathrm{CH}_{2}\right), 1.62\left(\mathrm{~s}, 6 \mathrm{H}, \mathrm{C}=\mathrm{C}\left(\mathrm{CH}_{3}\right)_{2}\right), 1.23(\mathrm{~m}, 5 \mathrm{H}, \mathrm{Cy}-$ $\left.\mathrm{CH}_{2}\right), 0.91\left(\mathrm{~m}, 1 \mathrm{H}, \mathrm{SiH}_{2} \mathrm{CH}\right), 0.08\left(\mathrm{~s}, 9 \mathrm{H}, \mathrm{Si}\left(\mathrm{CH}_{3}\right)_{3}\right) .{ }^{13} \mathbf{C ~ N M R}\left(101 \mathrm{MHz}, \mathrm{CDCl}_{3}, 23{ }^{\circ} \mathrm{C}\right) \delta 213.46$ $(\mathrm{C}=C=\mathrm{C}), 79.74,79.27(C=\mathrm{C}=C), 28.92,27.93,26.95,22.54(\mathrm{Cy}-C), 18.91\left(\mathrm{C}=\mathrm{C}\left(\mathrm{CH}_{3}\right)_{2}\right),-0.43$ $\left(\mathrm{Si}\left(\mathrm{CH}_{3}\right)_{3}\right)$. EI-MS for $\mathrm{M}^{+}: \mathrm{m} / \mathrm{z} 252.19$.

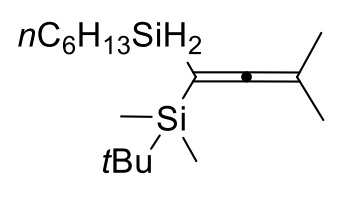

8c Colorless oil 8c (yield $87 \%$ ). ${ }^{1} \mathbf{H}$ NMR $\left(400 \mathrm{MHz}, \mathrm{CDCl}_{3}, 23{ }^{\circ} \mathrm{C}\right) \delta 3.97(\mathrm{~m}, 2 \mathrm{H}$, $\left.\mathrm{Si} H_{2}\right), 1.62\left(\mathrm{~s}, 6 \mathrm{H}, \mathrm{C}=\mathrm{C}\left(\mathrm{CH}_{3}\right)_{2}\right), 1.49-1.24\left(\mathrm{~m}, 8 \mathrm{H}, n \mathrm{Hex}-\mathrm{CH}_{2}\right), 0.90\left(\mathrm{~m}, 12 \mathrm{H}, n \mathrm{Hex}-\mathrm{CH}_{3}+\mathrm{C}\left(\mathrm{CH}_{3}\right)_{3}\right)$, $0.75\left(\mathrm{~m}, 2 \mathrm{H}, \mathrm{SiH}_{2} \mathrm{CH}_{2}\right), 0.06\left(\mathrm{~s}, 6 \mathrm{H}, \mathrm{Si}\left(\mathrm{CH}_{3}\right)_{2}\right) .{ }^{13} \mathbf{C ~ N M R}\left(101 \mathrm{MHz}, \mathrm{CDCl}_{3}, 23{ }^{\circ} \mathrm{C}\right) \delta 214.34(\mathrm{C}=\mathrm{C}=\mathrm{C})$, 79.65, $77.71(C=\mathrm{C}=C), 32.79,31.80,25.16,22.78\left(n \mathrm{Hex}-\mathrm{CH}_{2}\right), 26.71,17.78\left(C\left(\mathrm{CH}_{3}\right)_{3}\right), 18.90$ 
$\left(\mathrm{C}=\mathrm{C}\left(\mathrm{CH}_{3}\right)_{2}\right), 14.31\left(n \mathrm{Hex}-\mathrm{CH}_{3}\right), 10.57\left(\mathrm{SiH}_{2} \mathrm{CH}_{2}\right),-4.95\left(\mathrm{Si}\left(\mathrm{CH}_{3}\right)_{2}\right) . \mathbf{E I}-\mathbf{M S}$ for $\mathrm{M}^{+}: \mathrm{m} / \mathrm{z} 296.21$.

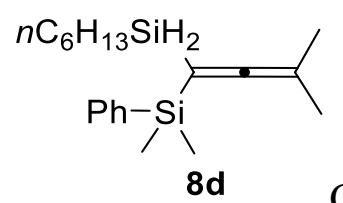

Colorless oil 8d (yield $86 \%$ ). ${ }^{1} \mathbf{H}$ NMR $\left(400 \mathrm{MHz}, \mathrm{CDCl}_{3}, 23{ }^{\circ} \mathrm{C}\right) \delta 7.54(\mathrm{~m}, 2 \mathrm{H}$, $\operatorname{Ar}-H), 7.36(\mathrm{~m}, 3 \mathrm{H}, \mathrm{Ar}-H), 3.93$ (br, 2H, $\left.\mathrm{Si} H_{2}\right), 1.64\left(\mathrm{~s}, 6 \mathrm{H}, \mathrm{C}=\mathrm{C}\left(\mathrm{CH}_{3}\right)_{2}\right), 1.43-1.20(\mathrm{~m}, 8 \mathrm{H}, n \mathrm{Hex}-$ $\left.\mathrm{CH}_{2}\right), 0.90$ (br, 3H, $\left.n \mathrm{Hex}-\mathrm{CH}_{3}\right), 0.67$ (m, 2H, $\left.\mathrm{SiH}_{2} \mathrm{CH}_{2}\right), 0.39$ (s, 6H, $\left.\mathrm{Si}\left(\mathrm{CH}_{3}\right)_{2}\right) .{ }^{13} \mathrm{C}$ NMR $(101 \mathrm{MHz}$, $\left.\mathrm{CDCl}_{3}, 23{ }^{\circ} \mathrm{C}\right) \delta 214.56(\mathrm{C}=C=\mathrm{C}), 139.02,133.88,129.04,127.74(\mathrm{Ar}-C), 80.67,79.13(C=\mathrm{C}=C)$, 32.63, 31.71, 25.04, $22.71\left(n \mathrm{Hex}-\mathrm{CH}_{2}\right), 18.82\left(\mathrm{C}=\mathrm{C}\left(\mathrm{CH}_{3}\right)_{2}\right), 14.27\left(n \mathrm{Hex}-\mathrm{CH}_{3}\right), 10.40\left(\mathrm{SiH}_{2} \mathrm{CH}_{2}\right)$, $-1.90\left(\mathrm{Si}\left(\mathrm{CH}_{3}\right)_{2}\right) .{ }^{29} \mathrm{Si} \mathbf{N M R}\left(79 \mathrm{MHz}, \mathrm{CDCl}_{3}, 23{ }^{\circ} \mathrm{C}\right) \delta-8.54\left(\mathrm{Si}\left(\mathrm{CH}_{3}\right)_{3}\right),-38.19\left(\mathrm{SiH}_{2} \mathrm{CH}_{2}\right) . \mathbf{E I}-\mathbf{M S}$ for $\mathrm{M}^{+}: \mathrm{m} / \mathrm{z} 316.17$.

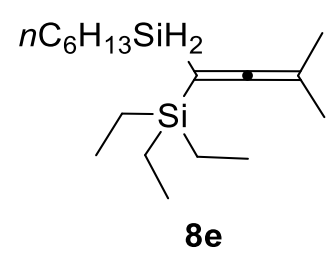

Colorless oil $8 \mathbf{e}$ (yield $85 \%$ ). ${ }^{1} \mathbf{H}$ NMR $\left(400 \mathrm{MHz}, \mathrm{CDCl}_{3}, 23{ }^{\circ} \mathrm{C}\right) \delta 3.94(\mathrm{~m}, 2 \mathrm{H}$, $\left.\mathrm{Si} H_{2}\right), 1.62\left(\mathrm{~s}, 6 \mathrm{H}, \mathrm{C}=\mathrm{C}\left(\mathrm{CH}_{3}\right)_{2}\right), 1.46-1.20\left(\mathrm{~m}, 8 \mathrm{H}, n \mathrm{Hex}-\mathrm{CH}_{2}\right), 0.94\left(\mathrm{t},{ }^{3} J_{\mathrm{HH}}=7.8 \mathrm{~Hz}, 9 \mathrm{H}, \mathrm{Et}-\mathrm{CH}_{3}\right)$, $0.89\left(\mathrm{t},{ }^{3} J_{\mathrm{HH}}=6.9 \mathrm{~Hz}, 3 \mathrm{H}, n \mathrm{Hex}-\mathrm{CH}_{3}\right), 0.74\left(\mathrm{~m}, 2 \mathrm{H}, \mathrm{SiH}_{2} \mathrm{CH}_{2}\right), 0.59\left(\mathrm{q},{ }^{3} J_{\mathrm{HH}}=7.9 \mathrm{~Hz}, 6 \mathrm{H}\right.$, $\left.\mathrm{Si}\left(\mathrm{CH}_{2} \mathrm{CH}_{3}\right)_{3}\right) .{ }^{13} \mathrm{C}$ NMR $\left(101 \mathrm{MHz}, \mathrm{CDCl}_{3}, 23{ }^{\circ} \mathrm{C}\right) \delta 214.04(\mathrm{C}=\mathrm{C}=\mathrm{C}), 79.36,76.72(\mathrm{C}=\mathrm{C}=\mathrm{C}), 32.75$, 31.78, 25.16, $22.75\left(n \mathrm{Hex}-\mathrm{CH}_{2}\right), 18.99\left(\mathrm{C}=\mathrm{C}\left(\mathrm{CH}_{3}\right)_{2}\right), 14.28\left(n \mathrm{Hex}-\mathrm{CH}_{3}\right), 10.49\left(\mathrm{SiH}_{2} \mathrm{CH}_{2}\right), 7.47,4.08$ (Et-C). ${ }^{29} \mathrm{Si}$ NMR $\left(79 \mathrm{MHz}, \mathrm{CDCl}_{3}, 23{ }^{\circ} \mathrm{C}\right) \delta 2.97\left(\mathrm{Si}\left(\mathrm{CH}_{2} \mathrm{CH}_{3}\right)_{3}\right),-38.42\left(\mathrm{SiH}_{2} \mathrm{CH}_{2}\right) . \mathbf{E I}-\mathbf{M S}$ for $\mathrm{M}^{+}$: $\mathrm{m} / \mathrm{z} 296.21$.

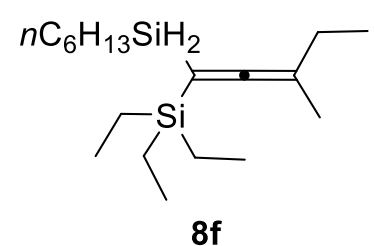

Colorless oil 8 f (yield $90 \%$ \%). ${ }^{\mathbf{1}} \mathbf{H}$ NMR $\left(400 \mathrm{MHz}, \mathrm{CDCl}_{3}, 23{ }^{\circ} \mathrm{C}\right) \delta 3.94\left(\mathrm{t},{ }^{3} \mathrm{~J}_{\mathrm{HH}}\right.$ $\left.=3.6 \mathrm{~Hz}, 2 \mathrm{H}, \mathrm{Si} H_{2}\right), 1.91\left(\mathrm{q},{ }^{3} J_{\mathrm{HH}}=7.4 \mathrm{~Hz}, 2 \mathrm{H}, \mathrm{C}=\mathrm{C}\left(\mathrm{CH}_{2} \mathrm{CH}_{3}\right)\right), 1.62\left(\mathrm{~s}, 3 \mathrm{H}, \mathrm{C}=\mathrm{C}\left(\mathrm{CH}_{3}\right)\right), 1.44-1.24$ $\left(\mathrm{m}, 8 \mathrm{H}, n \mathrm{Hex}-\mathrm{CH}_{2}\right), 0.99\left(\mathrm{t},{ }^{3} J_{\mathrm{HH}}=7.4 \mathrm{~Hz}, 3 \mathrm{H}, \mathrm{C}=\mathrm{C}\left(\mathrm{CH}_{2} \mathrm{CH}_{3}\right)\right), 0.94\left(\mathrm{t},{ }^{3} J_{\mathrm{HH}}=7.9 \mathrm{~Hz}, 9 \mathrm{H}\right.$, $\left.\mathrm{Si}\left(\mathrm{CH}_{2} \mathrm{CH}_{3}\right)_{3}\right), 0.89\left(\mathrm{t},{ }^{3} \mathrm{~J}_{\mathrm{HH}}=6.8 \mathrm{~Hz}, 3 \mathrm{H}, n \mathrm{Hex}-\mathrm{CH} \mathrm{H}_{3}\right), 0.75\left(\mathrm{~m}, 2 \mathrm{H}, \mathrm{SiH}_{2} \mathrm{CH}_{2}\right), 0.61\left(\mathrm{q},{ }^{3} J_{\mathrm{HH}}=7.8 \mathrm{~Hz}\right.$, $\left.6 \mathrm{H}, \mathrm{Si}\left(\mathrm{CH}_{2} \mathrm{CH}_{3}\right)_{3}\right) .{ }^{13} \mathrm{C}$ NMR $\left(101 \mathrm{MHz}, \mathrm{CDCl}_{3}, 23{ }^{\circ} \mathrm{C}\right) \delta 213.30(\mathrm{C}=\mathrm{C}=\mathrm{C}), 85.81,78.44(\mathrm{C}=\mathrm{C}=\mathrm{C})$, 
32.77, 31.76, 25.18, $22.74\left(n \mathrm{Hex}-\mathrm{CH}_{2}\right), 25.86,12.82\left(\mathrm{C}=\mathrm{C}\left(\mathrm{CH}_{2} \mathrm{CH}_{3}\right)\right), 17.42\left(\mathrm{C}=\mathrm{C}\left(\mathrm{CH}_{3}\right)\right), 14.28$ $\left(n \mathrm{Hex}-\mathrm{CH}_{3}\right), 10.50\left(\mathrm{SiH}_{2} \mathrm{CH}_{2}\right), 7.50,4.05\left(\mathrm{Si}\left(\mathrm{CH}_{2} \mathrm{CH}_{3}\right)_{3}\right) . \mathbf{E I}-\mathbf{M S}$ for $\mathrm{M}^{+}: \mathrm{m} / \mathrm{z} 310.23$.

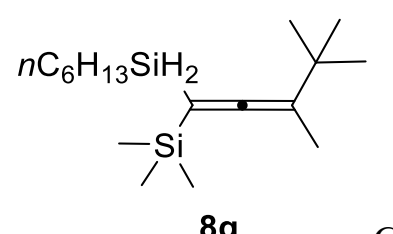

8g Colorless oil $\mathbf{8 g}$ (yield $91 \%)$. ${ }^{\mathbf{1}} \mathbf{H} \mathbf{N M R}\left(400 \mathrm{MHz}, \mathrm{CDCl}_{3}, 23{ }^{\circ} \mathrm{C}\right) \delta 3.96\left(\mathrm{t},{ }^{3} J_{\mathrm{HH}}\right.$ $\left.=3.4 \mathrm{~Hz}, 2 \mathrm{H}, \mathrm{Si} H_{2}\right), 1.62\left(\mathrm{~s}, 3 \mathrm{H}, \mathrm{C}=\mathrm{C}\left(\mathrm{CH}_{3}\right)\right), 1.45-1.26\left(\mathrm{~m}, 8 \mathrm{H}, n \mathrm{Hex}-\mathrm{CH}_{2}\right), 1.03\left(\mathrm{~s}, 9 \mathrm{H}, \mathrm{C}\left(\mathrm{CH}_{3}\right)_{3}\right)$, $0.89\left(\mathrm{t},{ }^{3} J_{\mathrm{HH}}=6.8 \mathrm{~Hz}, 3 \mathrm{H}, n \mathrm{Hex}-\mathrm{CH}_{3}\right), 0.77\left(\mathrm{~m}, 2 \mathrm{H}, \mathrm{SiH}_{2} \mathrm{CH}_{2}\right), 0.10\left(\mathrm{~s}, 9 \mathrm{H}, \mathrm{Si}\left(\mathrm{CH}_{3}\right)_{3}\right) .{ }^{13} \mathbf{C} \mathbf{N M R}(101$ $\left.\mathrm{MHz}, \mathrm{CDCl}_{3}, 23{ }^{\circ} \mathrm{C}\right) \delta 211.55(\mathrm{C}=C=\mathrm{C}), 94.00,81.56(C=\mathrm{C}=C), 32.78,31.79,25.29,22.77(n$ Hex$\left.\mathrm{CH}_{2}\right), 32.30,29.52\left(\mathrm{C}\left(\mathrm{CH}_{3}\right)_{3}\right), 13.56\left(\mathrm{C}=\mathrm{C}\left(\mathrm{CH}_{3}\right)\right), 14.28\left(n \mathrm{Hex}-\mathrm{CH}_{3}\right), 10.51\left(\mathrm{SiH}_{2} \mathrm{CH}_{2}\right),-0.48$ $\left(\mathrm{Si}\left(\mathrm{CH}_{3}\right)_{3}\right)$. EI-MS for $\mathrm{M}^{+}: \mathrm{m} / \mathrm{z} 296.20$.

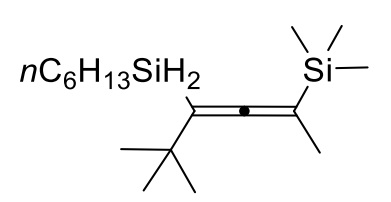

8h Colorless oil $\mathbf{8 h}$ (yield $94 \%)$. ${ }^{\mathbf{1}} \mathbf{H} \mathbf{~ N M R}\left(400 \mathrm{MHz}, \mathrm{CDCl}_{3}, 23{ }^{\circ} \mathrm{C}\right) \delta 4.03(\mathrm{~m}, 1 \mathrm{H}$, $\left.\mathrm{Si} H_{2}\right), 4.00\left(\mathrm{~m}, 1 \mathrm{H}, \mathrm{Si} H_{2}\right), 1.64\left(\mathrm{~s}, 3 \mathrm{H}, \mathrm{C}=\mathrm{C}\left(\mathrm{CH}_{3}\right)\right), 1.43-1.25\left(\mathrm{~m}, 8 \mathrm{H}, n \mathrm{Hex}-\mathrm{CH}_{2}\right), 1.08(\mathrm{~s}, 9 \mathrm{H}$, $\left.\mathrm{C}\left(\mathrm{CH}_{3}\right)_{3}\right), 0.89\left(\mathrm{t},{ }^{3} J_{\mathrm{HH}}=6.8 \mathrm{~Hz}, 3 \mathrm{H}, n \mathrm{Hex}-\mathrm{CH}_{3}\right), 0.72\left(\mathrm{~m}, 2 \mathrm{H}, \mathrm{SiH}_{2} \mathrm{CH}_{2}\right), 0.08\left(\mathrm{~s}, 9 \mathrm{H}, \mathrm{Si}\left(\mathrm{CH}_{3}\right)_{3}\right) .{ }^{13} \mathrm{C}$ NMR $\left(101 \mathrm{MHz}, \mathrm{CDCl}_{3}, 23{ }^{\circ} \mathrm{C}\right) \delta 205.63(\mathrm{C}=C=\mathrm{C}), 92.79,85.11(C=\mathrm{C}=C), 33.91\left(C\left(\mathrm{CH}_{3}\right)_{3}\right), 32.84$, 31.78, 25.38, $22.77\left(n \mathrm{Hex}-\mathrm{CH}_{2}\right), 31.08\left(\mathrm{C}_{\left.\left(\mathrm{CH}_{3}\right)_{3}\right),} 14.97\left(\mathrm{C}=\mathrm{C}\left(\mathrm{CH}_{3}\right)\right), 14.29\left(n \mathrm{Hex}-\mathrm{CH}_{3}\right), 10.72\right.$ $\left(\mathrm{SiH}_{2} \mathrm{CH}_{2}\right),-1.53\left(\mathrm{Si}\left(\mathrm{CH}_{3}\right)_{3}\right) .{ }^{29} \mathrm{Si} \mathbf{N M R}\left(79 \mathrm{MHz}, \mathrm{CDCl}_{3}, 23{ }^{\circ} \mathrm{C}\right) \delta-3.38\left(\mathrm{Si}\left(\mathrm{CH}_{3}\right)_{3}\right),-39.60$ $\left(\mathrm{SiH}_{2} \mathrm{CH}_{2}\right) . \mathbf{E I}-\mathbf{M S}$ for $\mathrm{M}^{+}: \mathrm{m} / \mathrm{z} 296.21$.

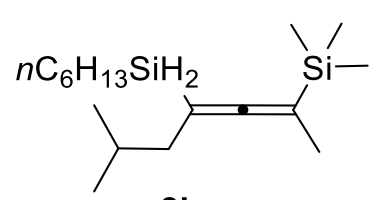

$8 \mathbf{i}$

Colorless oil $8 \mathbf{i}$ (yield $93 \%$ ). ${ }^{1} \mathbf{H}$ NMR $\left(400 \mathrm{MHz}, \mathrm{CDCl}_{3}, 23{ }^{\circ} \mathrm{C}\right) \delta 3.92(\mathrm{~m}, 2 \mathrm{H}$, $\left.\mathrm{Si} H_{2}\right), 1.85\left(\mathrm{~d},{ }^{3} J_{\mathrm{HH}}=6.9 \mathrm{~Hz}, 2 \mathrm{H}, i \operatorname{PrCH} H_{2}\right), 1.68(\mathrm{~m}, 1 \mathrm{H}, i \mathrm{Pr}-\mathrm{CH}), 1.63\left(\mathrm{~s}, 3 \mathrm{H}, \mathrm{C}=\mathrm{C}\left(\mathrm{CH}_{3}\right)\right), 1.45-1.24$ $\left(\mathrm{m}, 8 \mathrm{H}, n \mathrm{Hex}-\mathrm{CH}_{2}\right), 0.91\left(\mathrm{~d},{ }^{3} J_{\mathrm{HH}}=6.7 \mathrm{~Hz}, 6 \mathrm{H}, i \mathrm{Pr}-\mathrm{CH}_{3}\right), 0.89\left(\mathrm{t},{ }^{3} J_{\mathrm{HH}}=7.0 \mathrm{~Hz}, 3 \mathrm{H}, n \mathrm{Hex}-\mathrm{CH}_{3}\right), 0.73$ (m, 2H, $\left.\mathrm{SiH}_{2} \mathrm{CH}_{2}\right), 0.07\left(\mathrm{~s}, 9 \mathrm{H}, \mathrm{Si}\left(\mathrm{CH}_{3}\right)_{3}\right) .{ }^{13} \mathbf{C ~ N M R}\left(101 \mathrm{MHz}, \mathrm{CDCl}_{3}, 23{ }^{\circ} \mathrm{C}\right) \delta 207.56(\mathrm{C}=\mathrm{C}=\mathrm{C})$, 82.93, $80.18(C=\mathrm{C}=C), 40.79(i \mathrm{PrCH} 2), 32.77,31.75,25.30,22.74\left(n \mathrm{Hex}-\mathrm{CH}_{2}\right), 28.44(i \mathrm{Pr}-\mathrm{CH}), 22.86$, 
$22.65\left(i \mathrm{Pr}-\mathrm{CH}_{3}\right), 14.85\left(\mathrm{C}=\mathrm{C}\left(\mathrm{CH}_{3}\right)\right), 14.26\left(n \mathrm{Hex}-\mathrm{CH}_{3}\right), 9.96\left(\mathrm{SiH}_{2} \mathrm{CH}_{2}\right),-1.43\left(\mathrm{Si}\left(\mathrm{CH}_{3}\right)_{3}\right) . \mathbf{E I}-\mathbf{M S}$ for $\mathrm{M}^{+}: \mathrm{m} / \mathrm{z} 296.21$.

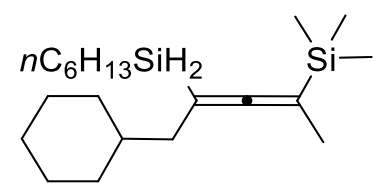

8j Colorless oil 8j (yield $87 \%$ ). ${ }^{1} \mathbf{H}$ NMR (400 MHz, $\left.\mathrm{CDCl}_{3}, 23{ }^{\circ} \mathrm{C}\right) \delta 3.91\left(\mathrm{t},{ }^{3} J_{\mathrm{HH}}\right.$ $\left.=3.6 \mathrm{~Hz}, 2 \mathrm{H}, \mathrm{Si} H_{2}\right), 1.85-1.65\left(\mathrm{~m}, 8 \mathrm{H}, \mathrm{CyCH}_{2}+\mathrm{Cy}-\mathrm{CH}_{2}+\mathrm{Cy}-\mathrm{CH}\right), 1.63\left(\mathrm{~s}, 3 \mathrm{H}, \mathrm{C}=\mathrm{C}\left(\mathrm{CH} H_{3}\right)\right)$, 1.44-1.25 (m, 8H, $\left.n \mathrm{Hex}-\mathrm{CH}_{2}\right), 1.17\left(\mathrm{~m}, 5 \mathrm{H}, \mathrm{Cy}-\mathrm{CH}_{2}\right), 0.89\left(\mathrm{t},{ }^{3} \mathrm{~J}_{\mathrm{HH}}=6.8 \mathrm{~Hz}, 3 \mathrm{H}, n \mathrm{Hex}-\mathrm{CH}_{3}\right), 0.73$ (m, 2H, $\left.\mathrm{SiH}_{2} \mathrm{CH}_{2}\right), 0.07$ (s, 9H, $\left.\mathrm{Si}\left(\mathrm{CH}_{3}\right)_{3}\right) .{ }^{13} \mathrm{C}$ NMR (101 MHz, $\left.\mathrm{CDCl}_{3}, 23{ }^{\circ} \mathrm{C}\right) \delta 207.51(\mathrm{C}=\mathrm{C}=\mathrm{C})$, 82.85, $79.71(C=\mathrm{C}=C), 39.14,37.99,33.71,33.36,29.88,26.84,26.50\left(\mathrm{CyCH}_{2}-C\right), 32.77,31.75,25.30$, $22.75\left(n \mathrm{Hex}-\mathrm{CH}_{2}\right), 14.86\left(\mathrm{C}=\mathrm{C}\left(\mathrm{CH}_{3}\right)\right), 14.27\left(n \mathrm{Hex}-\mathrm{CH}_{3}\right), 9.98\left(\mathrm{SiH}_{2} \mathrm{CH}_{2}\right),-1.45\left(\mathrm{Si}\left(\mathrm{CH}_{3}\right)_{3}\right) . \mathbf{E I}-\mathbf{M S}$ for $\mathrm{M}^{+}: \mathrm{m} / \mathrm{z} 336.23$.

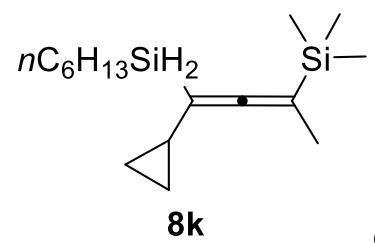

Colorless oil 8k (yield $89 \%$ ). ${ }^{1} \mathbf{H}$ NMR $\left(400 \mathrm{MHz}, \mathrm{CDCl}_{3}, 23{ }^{\circ} \mathrm{C}\right) \delta 3.97\left(\mathrm{t},{ }^{3} \mathrm{~J}_{\mathrm{HH}}\right.$ $\left.=3.2 \mathrm{~Hz}, 2 \mathrm{H}, \mathrm{Si} H_{2}\right), 1.62\left(\mathrm{~s}, 3 \mathrm{H}, \mathrm{C}=\mathrm{C}\left(\mathrm{CH}_{3}\right)\right), 1.44\left(\mathrm{~m}, 2 \mathrm{H}, n \mathrm{Hex}-\mathrm{CH}_{2}\right), 1.29\left(\mathrm{~m}, 6 \mathrm{H}, n \mathrm{Hex}-\mathrm{CH}_{2}\right), 1.09$ $\left(\mathrm{m}, 1 \mathrm{H}, c \mathrm{C}_{3} \mathrm{H}_{5}-\mathrm{CH}\right), 0.89\left(\mathrm{t},{ }^{3} \mathrm{~J}_{\mathrm{HH}}=6.6 \mathrm{~Hz}, 3 \mathrm{H}, n \mathrm{Hex}-\mathrm{CH}_{3}\right), 0.78\left(\mathrm{~m}, 2 \mathrm{H}, \mathrm{SiH}_{2} \mathrm{CH}_{2}\right), 0.63(\mathrm{~m}, 2 \mathrm{H}$, $\left.c \mathrm{C}_{3} \mathrm{H}_{5}-\mathrm{CH}_{2}\right), 0.33\left(\mathrm{~m}, 2 \mathrm{H}, c \mathrm{C}_{3} \mathrm{H}_{5}-\mathrm{CH}_{2}\right), 0.05\left(\mathrm{~s}, 9 \mathrm{H}, \mathrm{Si}\left(\mathrm{CH}_{3}\right)_{3}\right) .{ }^{13} \mathbf{C} \mathbf{N M R}\left(101 \mathrm{MHz}, \mathrm{CDCl}_{3}, 23{ }^{\circ} \mathrm{C}\right) \delta$ $205.69(\mathrm{C}=C=\mathrm{C}), 86.14,85.19(C=\mathrm{C}=C), 32.76,31.74,25.31,22.75\left(n \mathrm{Hex}-C_{2}\right), 14.91\left(\mathrm{C}=\mathrm{C}\left(C \mathrm{H}_{3}\right)\right)$, $14.27\left(n \mathrm{Hex}_{-} \mathrm{CH}_{3}\right), 10.71,7.63,7.07\left(c \mathrm{C}_{3} \mathrm{H}_{5}-\mathrm{C}\right), 9.97\left(\mathrm{SiH}_{2} \mathrm{CH}_{2}\right),-1.53\left(\mathrm{Si}\left(\mathrm{CH}_{3}\right)_{3}\right)$. EI-MS for $\mathrm{M}^{+}$: $\mathrm{m} / \mathrm{z} 280.19$.

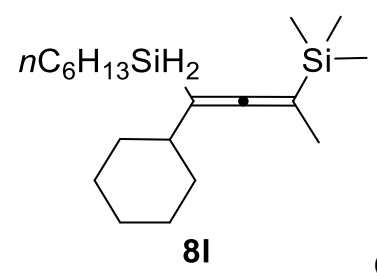

Colorless oil 81 (yield $92 \%$ ). ${ }^{1} \mathbf{H}$ NMR $\left(400 \mathrm{MHz}, \mathrm{CDCl}_{3}, 23{ }^{\circ} \mathrm{C}\right) \delta 3.94(\mathrm{~m}, 2 \mathrm{H}$, $\left.\mathrm{Si} H_{2}\right), 1.75\left(\mathrm{~m}, 6 \mathrm{H}, \mathrm{Cy}-\mathrm{CH}_{2}+\mathrm{Cy}-\mathrm{CH}\right), 1.63\left(\mathrm{~s}, 3 \mathrm{H}, \mathrm{C}=\mathrm{C}\left(\mathrm{CH}_{3}\right)\right), 1.45-1.23\left(\mathrm{~m}, 8 \mathrm{H}, n \mathrm{Hex}-\mathrm{CH}_{2}\right), 1.11$ (m, 5H, Cy-CH2), $0.88\left(\mathrm{br}, 3 \mathrm{H}, n \mathrm{Hex}-\mathrm{CH}_{3}\right), 0.72\left(\mathrm{~m}, 2 \mathrm{H}, \mathrm{SiH}_{2} \mathrm{CH}_{2}\right), 0.07\left(\mathrm{~s}, 9 \mathrm{H}, \mathrm{Si}\left(\mathrm{CH}_{3}\right)_{3}\right) .{ }^{13} \mathbf{C} \mathbf{N M R}$ 
$\left(101 \mathrm{MHz}, \mathrm{CDCl}_{3}, 23{ }^{\circ} \mathrm{C}\right) \delta 206.39(\mathrm{C}=C=\mathrm{C}), 87.50,84.57(C=\mathrm{C}=C), 39.79,34.17,33.87,29.87,26.79$, $26.38(\mathrm{Cy}-\mathrm{C}), 32.78,31.75,25.35,22.75\left(n \mathrm{Hex}-\mathrm{CH}_{2}\right), 14.97\left(\mathrm{C}=\mathrm{C}\left(\mathrm{CH}_{3}\right)\right), 14.27\left(n \mathrm{Hex}-\mathrm{CH}_{3}\right), 10.20$ $\left(\mathrm{SiH}_{2} \mathrm{CH}_{2}\right),-1.46\left(\mathrm{Si}\left(\mathrm{CH}_{3}\right)_{3}\right)$. EI-MS for $\mathrm{M}^{+}: \mathrm{m} / \mathrm{z} 322.21$.

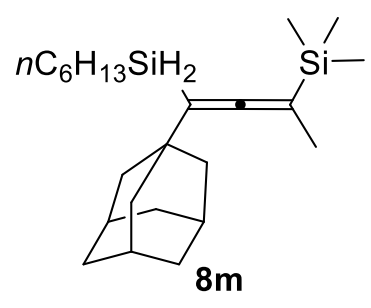

Colorless oil 8m (yield $86 \%$ ). ${ }^{\mathbf{1}} \mathbf{H}$ NMR (400 MHz, $\left.\mathrm{CDCl}_{3}, 23{ }^{\circ} \mathrm{C}\right) \delta 4.03(\mathrm{~m}$, 1H, $\left.\mathrm{Si} H_{2}\right), 3.98\left(\mathrm{~m}, 1 \mathrm{H}, \mathrm{Si} H_{2}\right), 1.97$ (br, 3H, adamantane- $\left.\mathrm{CH}\right), 1.66$ (br, 15H, adamantane- $\mathrm{CH}_{2}+$ $\left.\mathrm{C}=\mathrm{C}\left(\mathrm{CH}_{3}\right)\right), 1.45-1.25\left(\mathrm{~m}, 8 \mathrm{H}, n \mathrm{Hex}-\mathrm{CH}_{2}\right), 0.89\left(\mathrm{br}, 3 \mathrm{H}, n \mathrm{Hex}-\mathrm{CH}_{3}\right), 0.71\left(\mathrm{~m}, 2 \mathrm{H}, \mathrm{SiH}_{2} \mathrm{CH}_{2}\right), 0.08(\mathrm{~s}$, 9H, $\left.\mathrm{Si}\left(\mathrm{CH}_{3}\right)_{3}\right) .{ }^{13} \mathrm{C}$ NMR $\left(101 \mathrm{MHz}, \mathrm{CDCl}_{3}, 23{ }^{\circ} \mathrm{C}\right) \delta 206.03(\mathrm{C}=\mathrm{C}=\mathrm{C}), 93.23,85.04(\mathrm{C}=\mathrm{C}=\mathrm{C}), 43.58$, 36.99, 35.79, 29.27 (adamantane- $C), 32.83,31.77,25.43,22.77\left(n \mathrm{Hex}-\mathrm{CH}_{2}\right), 15.09\left(\mathrm{C}=\mathrm{C}\left(\mathrm{CH}_{3}\right)\right), 14.29$ $\left(n \mathrm{Hex}-\mathrm{CH}_{3}\right), 10.80\left(\mathrm{SiH}_{2} \mathrm{CH}_{2}\right),-1.44\left(\mathrm{Si}\left(\mathrm{CH}_{3}\right)_{3}\right)$. EI-MS for $\mathrm{M}^{+}: \mathrm{m} / \mathrm{z} 374.23$.

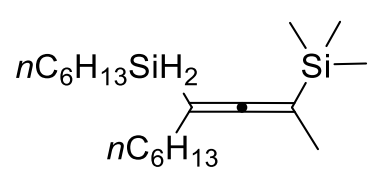

8n Colorless oil 8n (yield $84 \%)$. ${ }^{\mathbf{1}} \mathbf{H} \mathbf{N M R}\left(400 \mathrm{MHz}, \mathrm{CDCl}_{3}, 23{ }^{\circ} \mathrm{C}\right) \delta 3.92\left(\mathrm{t},{ }^{3} \mathrm{~J}_{\mathrm{HH}}\right.$ $\left.=3.3 \mathrm{~Hz}, 2 \mathrm{H}, \mathrm{Si} H_{2}\right), 1.95\left(\mathrm{~m}, 2 \mathrm{H}, \mathrm{C}=\mathrm{C}\left(n \mathrm{HexSiH}_{2}\right) \mathrm{CH}_{2}\right), 1.63\left(\mathrm{~s}, 3 \mathrm{H}, \mathrm{C}=\mathrm{C}\left(\mathrm{CH}_{3}\right)\right), 1.45-1.23(\mathrm{~m}, 16 \mathrm{H}$, $\left.n \mathrm{Hex}-\mathrm{CH}_{2}\right), 0.89\left(\mathrm{~m}, 6 \mathrm{H}, n \mathrm{Hex}-\mathrm{CH}_{3}\right), 0.73\left(\mathrm{~m}, 2 \mathrm{H}, \mathrm{SiH}_{2} \mathrm{CH}_{2}\right), 0.07\left(\mathrm{~s}, 9 \mathrm{H}, \mathrm{Si}\left(\mathrm{CH}_{3}\right)_{3}\right) .{ }^{13} \mathbf{C} \mathbf{N M R}(101$ $\left.\mathrm{MHz}, \mathrm{CDCl}_{3}, 23{ }^{\circ} \mathrm{C}\right) \delta 207.03(\mathrm{C}=C=\mathrm{C}), 83.58,81.32(C=\mathrm{C}=C), 32.77,31.93,25.30,22.75,31.75$, 30.95, 29.66, 29.20, $22.86\left(n \mathrm{Hex}-\mathrm{CH}_{2}\right), 14.83\left(\mathrm{C}=\mathrm{C}\left(\mathrm{CH}_{3}\right)\right), 14.28\left(n \mathrm{Hex}-\mathrm{CH}_{3}\right), 9.93\left(\mathrm{SiH}_{2} \mathrm{CH}_{2}\right),-1.49$ $\left(\mathrm{Si}\left(\mathrm{CH}_{3}\right)_{3}\right) . \mathbf{E I}-\mathbf{M S}$ for $\mathrm{M}^{+}: \mathrm{m} / \mathrm{z} 324.23$.

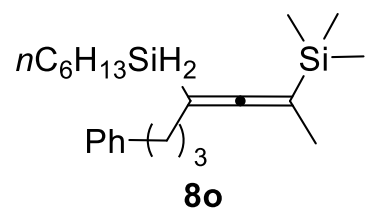

Colorless oil 80 (yield $83 \%$ ). ${ }^{1} \mathbf{H}$ NMR $\left(400 \mathrm{MHz}, \mathrm{CDCl}_{3}, 23{ }^{\circ} \mathrm{C}\right) \delta 7.30(\mathrm{~m}, 2 \mathrm{H}$, $\operatorname{Ar}-H), 7.22(\mathrm{~m}, 3 \mathrm{H}, \mathrm{Ar}-H), 3.97\left(\mathrm{t},{ }^{3} J_{\mathrm{HH}}=3.3 \mathrm{~Hz}, 2 \mathrm{H}, \mathrm{Si} H_{2}\right), 2.67\left(\mathrm{t},{ }^{3} J_{\mathrm{HH}}=7.7 \mathrm{~Hz}, 2 \mathrm{H}\right.$, $\mathrm{PhCH}_{2} \mathrm{CH}_{2} \mathrm{CH}_{2}$ ), $2.04\left(\mathrm{t},{ }^{3} \mathrm{~J}_{\mathrm{HH}}=7.4 \mathrm{~Hz}, 2 \mathrm{H}, \mathrm{PhCH}_{2} \mathrm{CH}_{2} \mathrm{CH}_{2}\right.$ ), 1.78 (quint, ${ }^{3} J_{\mathrm{HH}}=7.6 \mathrm{~Hz}, 2 \mathrm{H}$, $\left.\mathrm{PhCH}_{2} \mathrm{CH}_{2} \mathrm{CH}_{2}\right), 1.69\left(\mathrm{~s}, 3 \mathrm{H}, \mathrm{C}=\mathrm{C}\left(\mathrm{CH}_{3}\right)\right), 1.48-1.26\left(\mathrm{~m}, 8 \mathrm{H}, n \mathrm{Hex}-\mathrm{CH}_{2}\right), 0.91\left(\mathrm{t},{ }^{3} J_{\mathrm{HH}}=6.6 \mathrm{~Hz}, 3 \mathrm{H}\right.$, 
$\left.n \mathrm{Hex}-\mathrm{CH}_{3}\right), 0.76\left(\mathrm{~m}, 2 \mathrm{H}, \mathrm{SiH}_{2} \mathrm{CH}_{2}\right), 0.11\left(\mathrm{~s}, 9 \mathrm{H}, \mathrm{Si}\left(\mathrm{CH}_{3}\right)_{3}\right) .{ }^{13} \mathbf{C ~ N M R}\left(101 \mathrm{MHz}, \mathrm{CDCl}_{3}, 23{ }^{\circ} \mathrm{C}\right) \delta$ $207.02(\mathrm{C}=C=\mathrm{C}), 142.76,128.59,128.39,125.76(\operatorname{Ar}-C), 83.92,80.94(C=\mathrm{C}=C), 35.65,31.34,30.48$ $\left(\mathrm{PhCH}_{2} \mathrm{CH}_{2} \mathrm{CH}_{2}\right), 32.76,31.73,25.29,22.74\left(n \mathrm{Hex}-\mathrm{CH}_{2}\right), 14.90\left(\mathrm{C}=\mathrm{C}\left(\mathrm{CH}_{3}\right)\right), 14.28\left(n \mathrm{Hex}-\mathrm{CH}_{3}\right), 9.88$ $\left(\mathrm{SiH}_{2} \mathrm{CH}_{2}\right),-1.47\left(\mathrm{Si}\left(\mathrm{CH}_{3}\right)_{3}\right)$. EI-MS for $\mathrm{M}^{+}: \mathrm{m} / \mathrm{z} 358.25$.

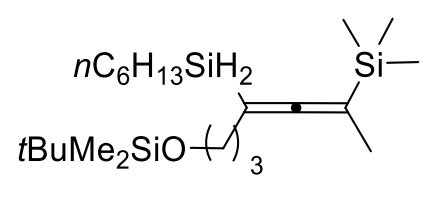

8p Colorless oil 8p (16 h, yield $85 \%)$. ${ }^{1} \mathbf{H}$ NMR $\left(400 \mathrm{MHz}, \mathrm{CDCl}_{3}, 23{ }^{\circ} \mathrm{C}\right) \delta$ $3.93\left(\mathrm{~m}, 2 \mathrm{H}, \mathrm{SiH} H_{2}\right), 3.63\left(\mathrm{t},{ }^{3} \mathrm{~J}_{\mathrm{HH}}=6.4 \mathrm{~Hz}, 2 \mathrm{H}, \mathrm{SiOCH} 2\right), 2.00\left(\mathrm{t},{ }^{3} \mathrm{~J}_{\mathrm{HH}}=7.1 \mathrm{~Hz}, 2 \mathrm{H}, \mathrm{SiOCH}_{2} \mathrm{CH}_{2} \mathrm{CH}_{2}\right)$, $1.66\left(\mathrm{~m}, 2 \mathrm{H}, \mathrm{SiOCH}_{2} \mathrm{CH}_{2} \mathrm{CH}_{2}\right), 1.63\left(\mathrm{~s}, 3 \mathrm{H}, \mathrm{C}=\mathrm{C}\left(\mathrm{CH}_{3}\right)\right), 1.45-1.25\left(\mathrm{~m}, 8 \mathrm{H}, n \mathrm{Hex}-\mathrm{CH}_{2}\right), 0.89(\mathrm{br}, 12 \mathrm{H}$, $\left.\mathrm{C}\left(\mathrm{CH}_{3}\right)_{3}+n \mathrm{Hex}-\mathrm{CH}_{3}\right), 0.74\left(\mathrm{~m}, 2 \mathrm{H}, \mathrm{SiH}_{2} \mathrm{CH}_{2}\right), 0.07\left(\mathrm{~s}, 9 \mathrm{H}, \mathrm{Si}\left(\mathrm{CH}_{3}\right)_{3}\right), 0.05\left(\mathrm{~s}, 6 \mathrm{H}, \mathrm{OSi}\left(\mathrm{CH}_{3}\right)_{2}\right) .{ }^{13} \mathrm{C}$ NMR $\left(101 \mathrm{MHz}, \mathrm{CDCl}_{3}, 23{ }^{\circ} \mathrm{C}\right) \delta 206.88(\mathrm{C}=C=\mathrm{C}), 84.03,80.95(C=\mathrm{C}=C), 62.98,32.82,27.07$, $\left(\mathrm{SiOCH}_{2} \mathrm{CH}_{2} \mathrm{CH}_{2}\right), \quad 32.76, \quad 31.73,25.28,22.74\left(n \mathrm{Hex}-\mathrm{CH}_{2}\right), 26.13,18.51\left(\mathrm{C}\left(\mathrm{CH}_{3}\right)_{3}\right), 14.82$

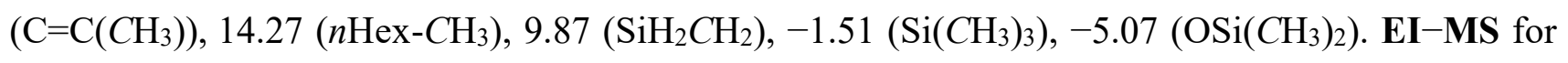
$\mathrm{M}^{+}: \mathrm{m} / \mathrm{z} 412.27$.

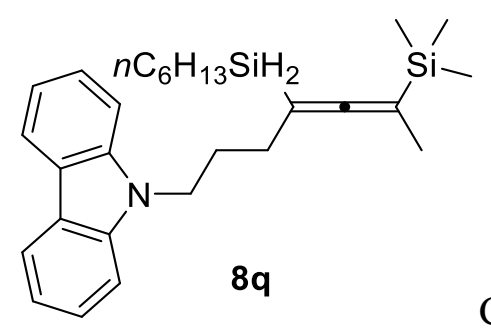

Colorless oil $\mathbf{8 q}$ (yield $92 \%$ ). ${ }^{1} \mathbf{H}$ NMR (400 $\left.\mathrm{MHz}, \mathrm{CDCl}_{3}, 23{ }^{\circ} \mathrm{C}\right) \delta 7.93$ $\left(\mathrm{d},{ }^{3} J_{\mathrm{HH}}=7.8 \mathrm{~Hz}, 2 \mathrm{H}, \operatorname{Ar}-H\right), 7.26(\mathrm{~m}, 4 \mathrm{H}, \mathrm{Ar}-H), 7.05(\mathrm{~m}, 2 \mathrm{H}, \operatorname{Ar}-H), 4.16\left(\mathrm{t},{ }^{3} J_{\mathrm{HH}}=6.8 \mathrm{~Hz}, 2 \mathrm{H}\right.$, $\left.\mathrm{NCH}_{2}\right), 3.78\left(\mathrm{~m}, 2 \mathrm{H}, \mathrm{Si} H_{2}\right), 1.90\left(\mathrm{~m}, 4 \mathrm{H}, \mathrm{NCH}_{2} \mathrm{CH}_{2} \mathrm{CH}_{2}\right), 1.55\left(\mathrm{~s}, 3 \mathrm{H}, \mathrm{C}=\mathrm{C}\left(\mathrm{CH}_{3}\right)\right), 1.24-1.10(\mathrm{~m}, 8 \mathrm{H}$, $\left.n \mathrm{Hex}-\mathrm{CH}_{2}\right), 0.72\left(\mathrm{t},{ }^{3} \mathrm{~J}_{\mathrm{HH}}=6.6 \mathrm{~Hz}, 3 \mathrm{H}, n \mathrm{Hex}-\mathrm{CH}_{3}\right), 0.56\left(\mathrm{~m}, 2 \mathrm{H}, \mathrm{SiH}_{2} \mathrm{CH}_{2}\right),-0.07\left(\mathrm{~s}, 9 \mathrm{H}, \mathrm{Si}\left(\mathrm{CH}_{3}\right)_{3}\right)$. ${ }^{13} \mathrm{C}$ NMR (101 MHz, $\left.\mathrm{CDCl}_{3}, 23{ }^{\circ} \mathrm{C}\right) \delta 206.57(\mathrm{C}=\mathrm{C}=\mathrm{C}), 140.56,125.72,122.92,120.45,118.84$, $108.80(\mathrm{Ar}-\mathrm{C}), 84.79,80.48(C=\mathrm{C}=C), 42.70,32.75,31.69\left(\mathrm{NCH}_{2} \mathrm{CH}_{2} \mathrm{CH}_{2}\right), 28.37,28.32,25.24,22.73$ $\left(n \mathrm{Hex}-\mathrm{CH}_{2}\right), 15.01\left(\mathrm{C}=\mathrm{C}\left(\mathrm{CH}_{3}\right)\right), 14.28\left(n \mathrm{Hex}-\mathrm{CH}_{3}\right), 9.75\left(\mathrm{SiH}_{2} \mathrm{CH}_{2}\right),-1.46\left(\mathrm{Si}\left(\mathrm{CH}_{3}\right)_{3}\right)$. HRMS (EI) calcd for $\left\{[\mathrm{M}]^{+},\left[\mathrm{C}_{28} \mathrm{H}_{41} \mathrm{NSi}_{2}\right]^{+}\right\}: 447.2772$; found 447.2770 . 


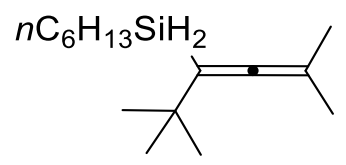

8r Colorless oil $8 \mathbf{r}(12 \mathrm{~h}$, yield $91 \%) .{ }^{\mathbf{1}} \mathbf{H} \mathbf{N M R}\left(400 \mathrm{MHz}, \mathrm{CDCl}_{3}, 23{ }^{\circ} \mathrm{C}\right) \delta 4.01(\mathrm{t}$, $\left.{ }^{3} J_{\mathrm{HH}}=3.2 \mathrm{~Hz}, 2 \mathrm{H}, \mathrm{Si} H_{2}\right), 1.66\left(\mathrm{~s}, 6 \mathrm{H}, \mathrm{C}=\mathrm{C}\left(\mathrm{CH}_{3}\right)_{2}\right), 1.43-1.23\left(\mathrm{~m}, 8 \mathrm{H}, n \mathrm{Hex}-\mathrm{CH}_{2}\right), 1.07(\mathrm{~s}, 9 \mathrm{H}$, $\left.\mathrm{C}\left(\mathrm{CH}_{3}\right)_{3}\right), 0.89\left(\mathrm{t},{ }^{3} \mathrm{~J}_{\mathrm{HH}}=6.7 \mathrm{~Hz}, 3 \mathrm{H}, n \mathrm{Hex}-\mathrm{CH}_{3}\right), 0.71\left(\mathrm{~m}, 2 \mathrm{H}, \mathrm{SiH}_{2} \mathrm{CH}_{2}\right) .{ }^{13} \mathbf{C} \mathbf{N M R}\left(101 \mathrm{MHz}, \mathrm{CDCl}_{3}\right.$, $\left.23{ }^{\circ} \mathrm{C}\right) \delta 204.95(\mathrm{C}=C=\mathrm{C}), 99.60,90.83(C=\mathrm{C}=C), 34.80,30.79\left(C\left(\mathrm{CH}_{3}\right)_{3}\right), 32.87,31.82,25.34,22.79$ $\left(n \mathrm{Hex}-\mathrm{CH}_{2}\right), 20.14\left(\mathrm{C}=\mathrm{C}\left(\mathrm{CH}_{3}\right)_{2}\right), 14.30\left(n \mathrm{Hex}-\mathrm{CH}_{3}\right), 10.58\left(\mathrm{SiH}_{2} \mathrm{CH}_{2}\right) . \mathbf{E I}-\mathbf{M S}$ for $\mathrm{M}^{+}: \mathrm{m} / \mathrm{z} 238.17$.

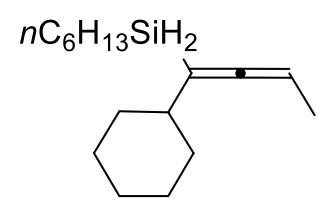

8s Colorless oil 8s $(6 \mathrm{~h}$, yield $92 \%) .{ }^{1} \mathbf{H}$ NMR $\left(400 \mathrm{MHz}, \mathrm{CDCl}_{3}, 23{ }^{\circ} \mathrm{C}\right) \delta 4.82$ (q, $\left.{ }^{3} J_{\mathrm{HH}}=6.9 \mathrm{~Hz}, 1 \mathrm{H}, \mathrm{C}=\mathrm{C}(H)\right), 3.94\left(\mathrm{~m}, 2 \mathrm{H}, \mathrm{Si} H_{2}\right), 1.88(\mathrm{~m}, 1 \mathrm{H}, \mathrm{Cy}-\mathrm{CH}), 1.76(\mathrm{~m}, 5 \mathrm{H}, \mathrm{Cy}-\mathrm{CH}), 1.62$ $\left(\mathrm{d},{ }^{3} J_{\mathrm{HH}}=6.9 \mathrm{~Hz}, 3 \mathrm{H}, \mathrm{C}=\mathrm{C}\left(\mathrm{CH}_{3}\right)\right), 1.38-1.20\left(\mathrm{~m}, 8 \mathrm{H}, n \mathrm{Hex}-\mathrm{CH}_{2}\right), 1.13\left(\mathrm{~m}, 5 \mathrm{H}, \mathrm{Cy}-\mathrm{CH}_{2}\right), 0.88\left(\mathrm{t},{ }^{3} J_{\mathrm{HH}}\right.$ $\left.=6.6 \mathrm{~Hz}, 3 \mathrm{H}, n \mathrm{Hex}-\mathrm{CH}_{3}\right), 0.75\left(\mathrm{~m}, 2 \mathrm{H}, \mathrm{SiH}_{2} \mathrm{CH}_{2}\right) .{ }^{13} \mathbf{C ~ N M R}\left(101 \mathrm{MHz}, \mathrm{CDCl}_{3}, 23{ }^{\circ} \mathrm{C}\right) \delta 207.45$ $(\mathrm{C}=C=\mathrm{C}), 95.57,80.96(C=\mathrm{C}=C), 40.24,33.54,33.43,29.86,26.63,26.30(\mathrm{Cy}-C), 32.67,31.71,25.19$, $22.72\left(n \mathrm{Hex}-\mathrm{CH}_{2}\right), 14.28\left(n \mathrm{Hex}-\mathrm{CH}_{3}\right), 14.01\left(\mathrm{C}=\mathrm{C}\left(\mathrm{CH}_{3}\right)\right), 9.86\left(\mathrm{SiH}_{2} \mathrm{CH}_{2}\right) . \mathbf{E I}-\mathbf{M S}$ for $\mathrm{M}^{+}: \mathrm{m} / \mathrm{z}$ 250.19 .

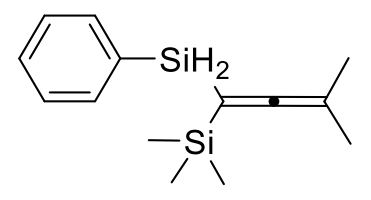

8t Colorless oil 8t (yield $87 \%$ ). ${ }^{\mathbf{1}} \mathbf{H} \mathbf{N M R}\left(400 \mathrm{MHz}, \mathrm{CDCl}_{3}, 23{ }^{\circ} \mathrm{C}\right) \delta 7.59\left(\mathrm{~d},{ }^{3} J_{\mathrm{HH}}\right.$ $=7.4 \mathrm{~Hz}, 2 \mathrm{H}, \mathrm{Ar}-\mathrm{H}), 7.39(\mathrm{~m}, 3 \mathrm{H}, \mathrm{Ar}-\mathrm{H}), 4.62\left(\mathrm{~s}, 2 \mathrm{H}, \mathrm{Si} H_{2}\right), 1.64\left(\mathrm{~s}, 6 \mathrm{H}, \mathrm{C}=\mathrm{C}\left(\mathrm{CH}_{3}\right)_{2}\right), 0.09(\mathrm{~s}, 9 \mathrm{H}$, $\left.\mathrm{Si}\left(\mathrm{CH}_{3}\right)_{3}\right) .{ }^{13} \mathrm{C}$ NMR $\left(101 \mathrm{MHz}, \mathrm{CDCl}_{3}, 23{ }^{\circ} \mathrm{C}\right) \delta 214.83(\mathrm{C}=\mathrm{C}=\mathrm{C}), 135.43,133.07,129.62,127.95$ $(\mathrm{Ar}-\mathrm{C}), 80.68,80.26(C=\mathrm{C}=C), 18.84\left(\mathrm{C}=\mathrm{C}\left(\mathrm{CH}_{3}\right)_{2}\right),-0.40\left(\mathrm{Si}\left(\mathrm{CH}_{3}\right)_{3}\right)$. EI-MS for $\mathrm{M}^{+}: \mathrm{m} / \mathrm{z} 246.19$.

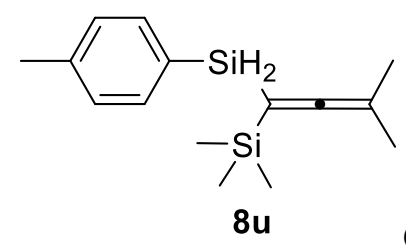

Colorless oil 8u (yield $85 \%$ ). ${ }^{\mathbf{1}} \mathbf{H}$ NMR $\left(400 \mathrm{MHz}, \mathrm{CDCl}_{3}, 23{ }^{\circ} \mathrm{C}\right) \delta 7.46(\mathrm{~d}$, 
$\left.{ }^{3} J_{\mathrm{HH}}=7.7 \mathrm{~Hz}, 2 \mathrm{H}, \mathrm{Ar}-H\right), 7.18\left(\mathrm{~d},{ }^{3} J_{\mathrm{HH}}=7.5 \mathrm{~Hz}, 2 \mathrm{H}, \mathrm{Ar}-H\right), 4.57\left(\mathrm{~s}, 2 \mathrm{H}, \mathrm{Si} H_{2}\right), 2.36(\mathrm{~s}, 3 \mathrm{H}, \mathrm{ArCH})$, $1.62\left(\mathrm{~s}, 6 \mathrm{H}, \mathrm{C}=\mathrm{C}\left(\mathrm{CH}_{3}\right)_{2}\right), 0.06\left(\mathrm{~s}, 9 \mathrm{H}, \mathrm{Si}\left(\mathrm{CH}_{3}\right)_{3}\right) .{ }^{13} \mathbf{C} \mathbf{N M R}\left(101 \mathrm{MHz}, \mathrm{CDCl}_{3}, 23{ }^{\circ} \mathrm{C}\right) \delta 214.70$ $(\mathrm{C}=C=\mathrm{C}), 139.53,135.45,129.29,128.80(\mathrm{Ar}-C), 80.55,80.42(C=\mathrm{C}=C), 21.70\left(\mathrm{ArCH}_{3}\right), 18.87$ $\left(\mathrm{C}=\mathrm{C}\left(\mathrm{CH}_{3}\right)_{2}\right),-0.38\left(\mathrm{Si}\left(\mathrm{CH}_{3}\right)_{3}\right)$. EI-MS for $\mathrm{M}^{+}: \mathrm{m} / \mathrm{z} 260.15$.

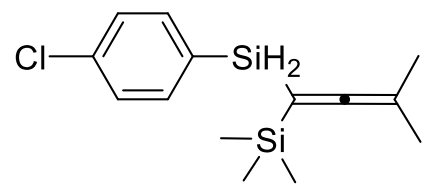

8v Colorless oil 8v (yield $81 \%$ ). ${ }^{\mathbf{1}} \mathbf{H} \mathbf{N M R}\left(400 \mathrm{MHz}, \mathrm{CDCl}_{3}, 23{ }^{\circ} \mathrm{C}\right) \delta 7.49(\mathrm{~d}$, $\left.{ }^{3} J_{\mathrm{HH}}=7.7 \mathrm{~Hz}, 2 \mathrm{H}, \operatorname{Ar}-H\right), 7.33\left(\mathrm{~d},{ }^{3} J_{\mathrm{HH}}=7.5 \mathrm{~Hz}, 2 \mathrm{H}, \mathrm{Ar}-H\right), 4.57\left(\mathrm{~s}, 2 \mathrm{H}, \mathrm{Si} H_{2}\right), 1.60(\mathrm{~s}, 6 \mathrm{H}$, $\left.\mathrm{C}=\mathrm{C}\left(\mathrm{CH}_{3}\right)_{2}\right), 0.06\left(\mathrm{~s}, 9 \mathrm{H}, \mathrm{Si}\left(\mathrm{CH}_{3}\right)_{3}\right) .{ }^{13} \mathrm{C} \mathbf{N M R}\left(101 \mathrm{MHz}, \mathrm{CDCl}_{3}, 23{ }^{\circ} \mathrm{C}\right) \delta 215.01(\mathrm{C}=\mathrm{C}=\mathrm{C}), 136.76$, 135.66, 131.44, $128.22(\mathrm{Ar}-\mathrm{C}), 80.99,80.03(\mathrm{C}=\mathrm{C}=\mathrm{C}), 18.81\left(\mathrm{C}=\mathrm{C}\left(\mathrm{CH}_{3}\right)_{2}\right),-0.41\left(\mathrm{Si}\left(\mathrm{CH}_{3}\right)_{3}\right) . \mathbf{E I}-\mathbf{M S}$ for $\mathrm{M}^{+}: \mathrm{m} / \mathrm{z} 280.02$.

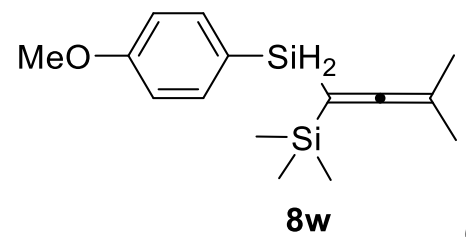

Colorless oil 8w (yield $83 \%)$. ${ }^{\mathbf{1}} \mathbf{H}$ NMR $\left(400 \mathrm{MHz}, \mathrm{CDCl}_{3}, 23{ }^{\circ} \mathrm{C}\right) \delta 7.49$ $\left(\mathrm{d},{ }^{3} J_{\mathrm{HH}}=8.5 \mathrm{~Hz}, 2 \mathrm{H}, \mathrm{Ar}-H\right), 6.91\left(\mathrm{~d},{ }^{3} J_{\mathrm{HH}}=8.5 \mathrm{~Hz}, 2 \mathrm{H}, \mathrm{Ar}-H\right), 4.57\left(\mathrm{~s}, 2 \mathrm{H}, \mathrm{Si} H_{2}\right), 3.82(\mathrm{~s}, 3 \mathrm{H}, \mathrm{OCH})_{3}$, $1.62\left(\mathrm{~s}, 6 \mathrm{H}, \mathrm{C}=\mathrm{C}\left(\mathrm{CH}_{3}\right)_{2}\right), 0.06\left(\mathrm{~s}, 9 \mathrm{H}, \mathrm{Si}\left(\mathrm{CH}_{3}\right)_{3}\right) .{ }^{13} \mathrm{C}$ NMR $\left(101 \mathrm{MHz}, \mathrm{CDCl}_{3}, 23{ }^{\circ} \mathrm{C}\right) \delta 214.61$ $(\mathrm{C}=C=\mathrm{C}), 160.96,136.91,123.66,113.77(\mathrm{Ar}-C), 80.64,80.55(C=\mathrm{C}=C), 55.15\left(\mathrm{OCH}_{3}\right), 18.88$ $\left(\mathrm{C}=\mathrm{C}\left(\mathrm{CH}_{3}\right)_{2}\right),-0.39\left(\mathrm{Si}\left(\mathrm{CH}_{3}\right)_{3}\right)$. EI-MS for $\mathrm{M}^{+}: \mathrm{m} / \mathrm{z} 276.12$.

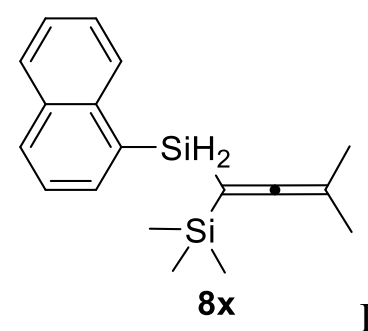

Pale yellow oil 8x (16 h, yield $89 \%) .{ }^{1} \mathbf{H}$ NMR $\left(400 \mathrm{MHz}, \mathrm{CDCl}_{3}, 23{ }^{\circ} \mathrm{C}\right) \delta 8.13$ (m, 1H, Ar- $H$ ), $7.91(\mathrm{~m}, 3 \mathrm{H}, \mathrm{Ar}-H), 7.51(\mathrm{~m}, 3 \mathrm{H}, \mathrm{Ar}-H), 4.96\left(\mathrm{~s}, 2 \mathrm{H}, \mathrm{Si} H_{2}\right), 1.56\left(\mathrm{~s}, 6 \mathrm{H}, \mathrm{C}=\mathrm{C}\left(\mathrm{C} H_{3}\right)_{2}\right)$, $0.06\left(\mathrm{~s}, 9 \mathrm{H}, \mathrm{Si}\left(\mathrm{CH}_{3}\right)_{3}\right) .{ }^{13} \mathrm{C}$ NMR $\left(101 \mathrm{MHz}, \mathrm{CDCl}_{3}, 23{ }^{\circ} \mathrm{C}\right) \delta 214.68(\mathrm{C}=\mathrm{C}=\mathrm{C}), 137.43,136.01,133.22$, $131.78,130.60,128.78,128.48,125.96,125.78,125.32(\mathrm{Ar}-C), 80.80,80.50(C=\mathrm{C}=C), 18.63$ 


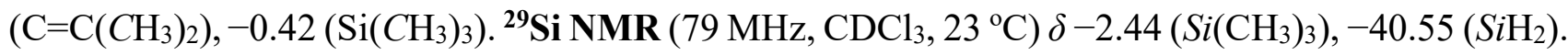
EI-MS for $\mathrm{M}^{+}: \mathrm{m} / \mathrm{z} 296.12$.

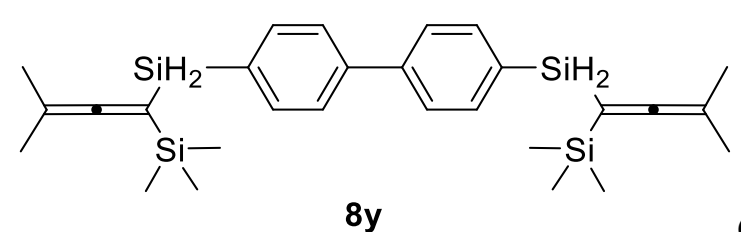

Colorless oil 8y (16 h, yield $85 \%$ ). ${ }^{\mathbf{1}} \mathbf{H}$ NMR (400 MHz, $\left.\mathrm{CDCl}_{3}, 23{ }^{\circ} \mathrm{C}\right) \delta 7.65(\mathrm{~m}, 8 \mathrm{H}, \mathrm{Ar}-\mathrm{H}), 4.64\left(\mathrm{~s}, 4 \mathrm{H}, \mathrm{Si} H_{2}\right), 1.63\left(\mathrm{~s}, 12 \mathrm{H}, \mathrm{C}=\mathrm{C}\left(\mathrm{CH}_{3}\right)_{2}\right), 0.10(\mathrm{~s}, 18 \mathrm{H}$, $\left.\mathrm{Si}\left(\mathrm{CH}_{3}\right)_{3}\right) .{ }^{13} \mathrm{C} \mathbf{N M R}\left(101 \mathrm{MHz}, \mathrm{CDCl}_{3}, 23{ }^{\circ} \mathrm{C}\right) \delta 214.94(\mathrm{C}=\mathrm{C}=\mathrm{C}), 142.07,135.95,132.14,126.62$ (Ar- $C), 80.79,80.25(C=\mathrm{C}=C), 18.87\left(\mathrm{C}=\mathrm{C}\left(\mathrm{CH}_{3}\right)_{2}\right),-0.35\left(\mathrm{Si}\left(\mathrm{CH}_{3}\right)_{3}\right)$. EI-MS for $\mathrm{M}^{+}: \mathrm{m} / \mathrm{z} 490.25$.

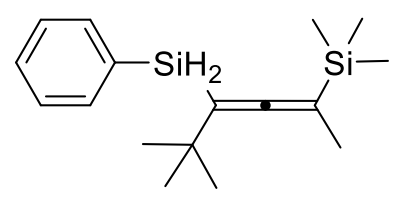

$8 \mathbf{z} \quad$ Colorless oil $8 \mathbf{z}$ (yield $84 \%$ ). ${ }^{\mathbf{1}} \mathbf{H}$ NMR $\left(400 \mathrm{MHz}, \mathrm{CDCl}_{3}, 23{ }^{\circ} \mathrm{C}\right) \delta 7.59(\mathrm{~m}$, 2H, Ar- $H$ ), 7.35 (m, 3H, Ar- $H), 4.72\left(\mathrm{~m}, 1 \mathrm{H}, \mathrm{PhSi} H_{2}\right), 4.63\left(\mathrm{~m}, 1 \mathrm{H}, \mathrm{PhSi} H_{2}\right), 1.57$ (s, 3H, C=C(CH3)), $1.10\left(\mathrm{~s}, 9 \mathrm{H}, \mathrm{C}\left(\mathrm{CH}_{3}\right)_{3}\right),-0.03\left(\mathrm{~s}, 9 \mathrm{H}, \mathrm{Si}\left(\mathrm{CH}_{3}\right)_{3}\right) .{ }^{13} \mathbf{C ~ N M R}\left(101 \mathrm{MHz}, \mathrm{CDCl}_{3}, 23{ }^{\circ} \mathrm{C}\right) \delta 206.88(\mathrm{C}=\mathrm{C}=\mathrm{C})$, 135.74, 133.56, 129.49, $\left.127.84(\mathrm{Ar}-\mathrm{C}), 92.73,85.59(\mathrm{C}=\mathrm{C}=C), 34.14\left(\mathrm{C}\left(\mathrm{CH}_{3}\right)_{3}\right), 31.22\left(\mathrm{C}_{(\mathrm{CH}}\right)_{3}\right)$, $14.80\left(\mathrm{C}=\mathrm{C}\left(\mathrm{CH}_{3}\right)\right),-1.74\left(\mathrm{Si}\left(\mathrm{CH}_{3}\right)_{3}\right) . \mathbf{E I}-\mathbf{M S}$ for $\mathrm{M}^{+}: \mathrm{m} / \mathrm{z} 288.22$.

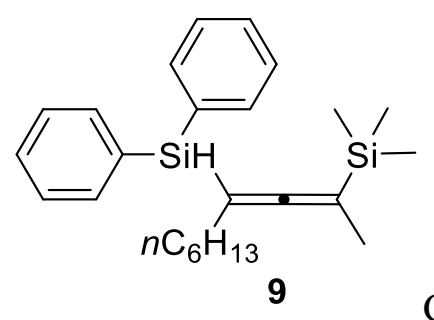

Colorless oil $9\left(60{ }^{\circ} \mathrm{C}, 16 \mathrm{~h}\right.$, yield $\left.84 \%\right) .{ }^{\mathbf{1}} \mathbf{H}$ NMR $\left(400 \mathrm{MHz}, \mathrm{CDCl}_{3}, 23{ }^{\circ} \mathrm{C}\right)$ $\delta 7.62(\mathrm{~m}, 4 \mathrm{H}, \mathrm{Ar}-H), 7.41$ (m, 6H, Ar- $H), 5.16(\mathrm{~s}, 1 \mathrm{H}, \mathrm{Ph} 2 \mathrm{Si} H), 2.06\left(\mathrm{~m}, 2 \mathrm{H}, \mathrm{C}=\mathrm{C}\left(\mathrm{Ph}_{2} \mathrm{SiH}\right) \mathrm{CH} \mathrm{H}_{2}\right), 1.63$ $\left(\mathrm{s}, 3 \mathrm{H}, \mathrm{C}=\mathrm{C}\left(\mathrm{CH}_{3}\right)\right), 1.49\left(\mathrm{~m}, 2 \mathrm{H}, n \mathrm{Hex}-\mathrm{CH}_{2}\right), 1.30\left(\mathrm{~m}, 6 \mathrm{H}, n \mathrm{Hex}-\mathrm{CH}_{2}\right), 0.91\left(\mathrm{t},{ }^{3} J_{\mathrm{HH}}=6.9 \mathrm{~Hz}, 3 \mathrm{H}\right.$, $\left.n \mathrm{Hex}-\mathrm{CH}_{3}\right),-0.05\left(\mathrm{~s}, 9 \mathrm{H}, \mathrm{Si}\left(\mathrm{CH}_{3}\right)_{3}\right) .{ }^{13} \mathrm{C} \mathbf{N M R}\left(101 \mathrm{MHz}, \mathrm{CDCl}_{3}, 23{ }^{\circ} \mathrm{C}\right) \delta 208.54(\mathrm{C}=\mathrm{C}=\mathrm{C}), 135.73$, 134.21, 129.59, $127.89(\mathrm{Ar}-C), 84.55,83.10(C=\mathrm{C}=C), 31.89,30.15,29.61,29.17,22.82\left(n \mathrm{Hex}-C_{\mathrm{H}}\right)$, $14.86\left(\mathrm{C}=\mathrm{C}\left(\mathrm{CH}_{3}\right)\right), 14.28\left(n \mathrm{Hex}-\mathrm{CH}_{3}\right),-1.77\left(\mathrm{Si}\left(\mathrm{CH}_{3}\right)_{3}\right) .{ }^{29} \mathrm{Si} \mathrm{NMR}\left(79 \mathrm{MHz}, \mathrm{CDCl}_{3}, 23{ }^{\circ} \mathrm{C}\right) \delta-3.17$ $\left(\mathrm{Si}\left(\mathrm{CH}_{3}\right)_{3}\right),-100.00\left(\mathrm{SiH}_{2}\right)$. EI-MS for $\mathrm{M}^{+}: \mathrm{m} / \mathrm{z} 392.24$. 


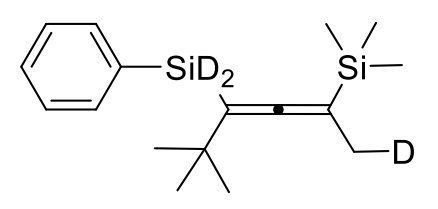

10 Colorless oil 10 (yield $90 \%$ ). ${ }^{1} \mathbf{H}$ NMR (400 MHz, $\left.\mathrm{CDCl}_{3}, 23{ }^{\circ} \mathrm{C}\right) \delta 7.58(\mathrm{~d}$, $\left.{ }^{3} J_{\mathrm{HH}}=7.3 \mathrm{~Hz}, 2 \mathrm{H}, \mathrm{Ar}-H\right), 7.35(\mathrm{~m}, 3 \mathrm{H}, \mathrm{Ar}-H), 1.55\left(\mathrm{~s}, 2 \mathrm{H}, \mathrm{C}=\mathrm{C}\left(\mathrm{CDH}_{2}\right)\right), 1.09\left(\mathrm{~s}, 9 \mathrm{H}, \mathrm{C}\left(\mathrm{CH}_{3}\right)_{3}\right),-0.04$ (s, 9H, $\left.\mathrm{Si}\left(\mathrm{CH}_{3}\right)_{3}\right) .{ }^{2} \mathbf{H}$ NMR $\left(92 \mathrm{MHz}, \mathrm{CHCl}_{3}, 23{ }^{\circ} \mathrm{C}\right) \delta 4.76\left(\mathrm{~s}, 1 \mathrm{H}, \mathrm{Si} D_{2}\right), 4.67\left(\mathrm{~s}, 1 \mathrm{H}, \mathrm{Si} D_{2}\right), 1.57(\mathrm{t}$, $\left.{ }^{2} J_{\mathrm{HD}}=2.1 \mathrm{~Hz}, 1 \mathrm{H}, \mathrm{CH}_{2} D\right) .{ }^{13} \mathbf{C}$ NMR $\left(101 \mathrm{MHz}, \mathrm{CDCl}_{3}, 23{ }^{\circ} \mathrm{C}\right) \delta 206.89(\mathrm{C}=C=\mathrm{C}), 135.73,133.51$, 129.48, $127.83(\mathrm{Ar}-C), 92.61,85.48(C=\mathrm{C}=C), 34.11,31.22\left(C\left(C \mathrm{H}_{3}\right)_{3}\right), 14.53\left(\mathrm{t},{ }^{1} J_{\mathrm{CD}}=19.7 \mathrm{~Hz}\right.$, $\left.\mathrm{C}=\mathrm{C}\left(C \mathrm{CH}_{2}\right)\right),-1.74\left(\mathrm{Si}\left(\mathrm{CH}_{3}\right)_{3}\right)$. HRMS (EI) Calcd for $\left\{[\mathrm{M}]^{+},\left[\mathrm{C}_{17} \mathrm{H}_{25} \mathrm{D}_{3} \mathrm{Si}\right]^{+}\right\}: 291.1912$; Found 291.1912. 


\section{Reactions of Silylallenes}

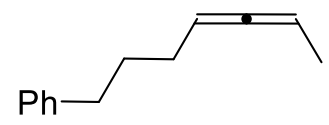

11 A Schlenk bottle was charged with TBAF (tetrabutylammoniumfluoride, 0.60 mmol, 3.0 eq) and THF $(1.0 \mathrm{~mL})$. And then 1,3-enyne $80(0.20 \mathrm{mmol})$ was added. After stirring at room temperature in Ar atmosphere for $0.5 \mathrm{~h}$, the mixture purified by flash column chromatography ( $n$-hexane) to give the product 11 as colorless oil (31 mg, yield $90 \%) .{ }^{1} \mathbf{H ~ N M R}\left(400 \mathrm{MHz}, \mathrm{CDCl}_{3}\right.$, $\left.23{ }^{\circ} \mathrm{C}\right) \delta 7.28(\mathrm{~m}, 2 \mathrm{H}, \mathrm{Ar}-H), 7.19(\mathrm{~m}, 3 \mathrm{H}, \mathrm{Ar}-H), 5.08(\mathrm{~m}, 2 \mathrm{H}, H \mathrm{C}=\mathrm{C}=\mathrm{CH}), 2.65\left(\mathrm{t},{ }^{3} J_{\mathrm{HH}}=7.7 \mathrm{~Hz}, 2 \mathrm{H}\right.$, $\mathrm{PhCH}_{2} \mathrm{CH}_{2} \mathrm{CH}_{2}$ ), 2.02 (m, 2H, $\mathrm{PhCH}_{2} \mathrm{CH}_{2} \mathrm{CH}_{2}$ ), 1.73 (quint, ${ }^{3} J_{\mathrm{HH}}=7.6 \mathrm{~Hz}, 2 \mathrm{H}, \mathrm{PhCH}_{2} \mathrm{CH}_{2} \mathrm{CH}_{2}$ ), 1.66 $\left(\mathrm{m}, 3 \mathrm{H}, \mathrm{C}=\mathrm{C}\left(\mathrm{CH}_{3}\right)\right) .{ }^{13} \mathrm{C}$ NMR $\left(101 \mathrm{MHz}, \mathrm{CDCl}_{3}, 23{ }^{\circ} \mathrm{C}\right) \delta 204.97(\mathrm{C}=\mathrm{C}=\mathrm{C}), 142.66,128.63,128.40$, $125.79(\mathrm{Ar}-C), 90.07,85.88(C=\mathrm{C}=C), 35.39,30.98,28.43\left(\mathrm{PhCH}_{2} \mathrm{CH}_{2} C \mathrm{H}_{2}\right), 14.79\left(\mathrm{C}=\mathrm{C}\left(C \mathrm{H}_{3}\right)\right)$. HRMS (EI) calcd for $\left\{[\mathrm{M}]^{+},\left[\mathrm{C}_{13} \mathrm{H}_{16}\right]^{+}\right\}: 172.1247$; found 172.1245 . 
Table S1. Substrate limitations. ${ }^{a}$

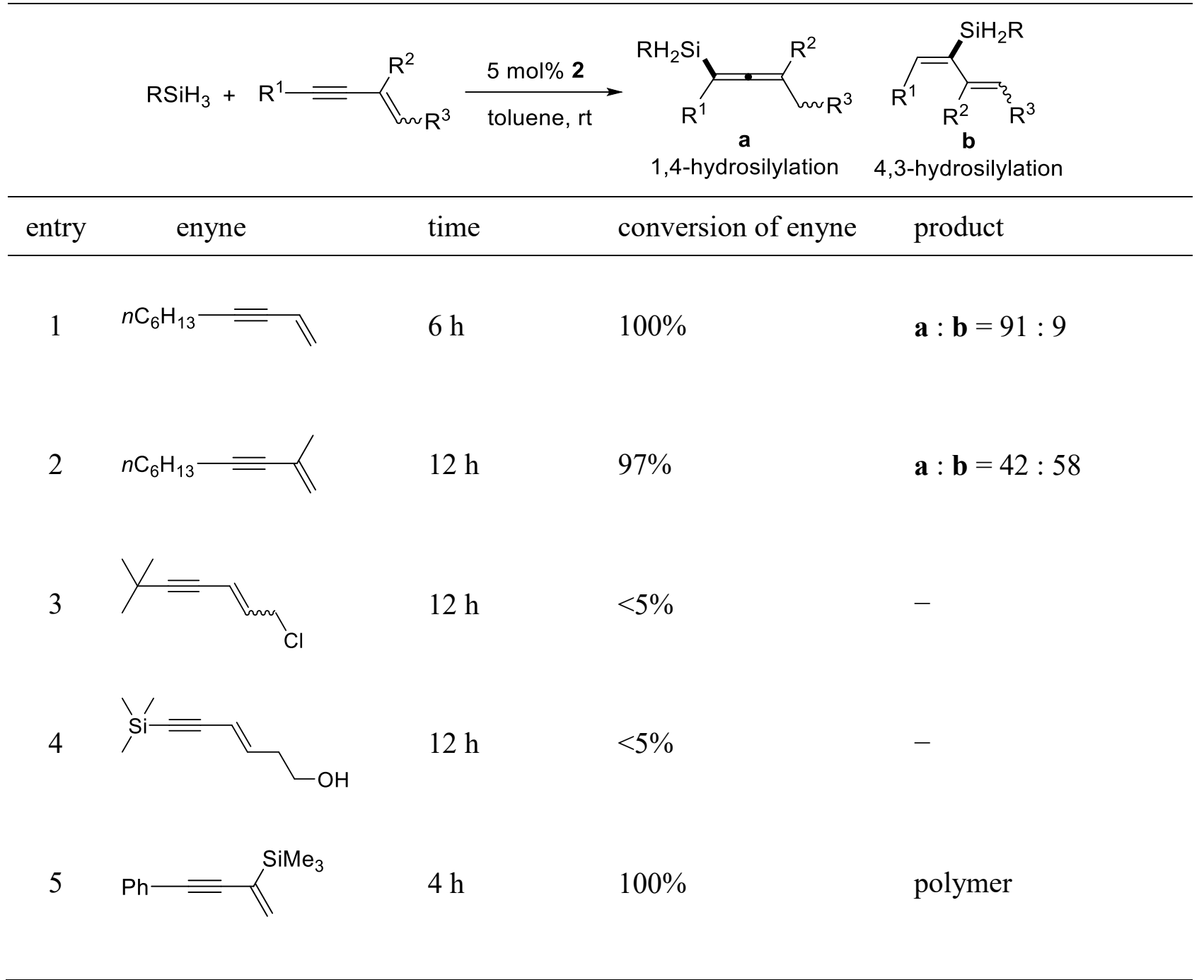

${ }^{a} \mathrm{RSiH}_{3}={ }_{n} \mathrm{C}_{6} \mathrm{H}_{13} \mathrm{SiH}_{3}$. Conversion of enyne and ratio of products a to $\mathbf{b}$ were determined by GC-MS and ${ }^{1} \mathrm{H}$ NMR of the crude reaction mixture. 


\section{DFT Calculations}

Geometry optimization and frequency analysis were performed using B3LYP functional ${ }^{\mathrm{S} 9}$ with DFT-D3(BJ) dispersion correction ${ }^{\mathrm{S} 10}$ and a mixed basis set of MWB46 ${ }^{\mathrm{S} 11}$ for lanthanum atom and 6$311 \mathrm{G}$ (d) for other atoms in the gas phase. Intrinsic reaction coordinate (IRC) $)^{\mathrm{S} 12}$ calculations for all transition states have been performed to confirm the transition states are connected to the right intermediates. The single point energies were then calculated using the PBE0 functional ${ }^{\mathrm{S} 13}$ and a basis set of ma-def2-TZVP ${ }^{\mathrm{S} 14, \mathrm{~S} 15}$ in toluene solvent with the SMD solvation model. ${ }^{\mathrm{S} 16}$ All of the computations were performed with Gaussian $16 .{ }^{\mathrm{S} 17}$ Computed structures were illustrated using CYLView. ${ }^{\text {S18 }}$
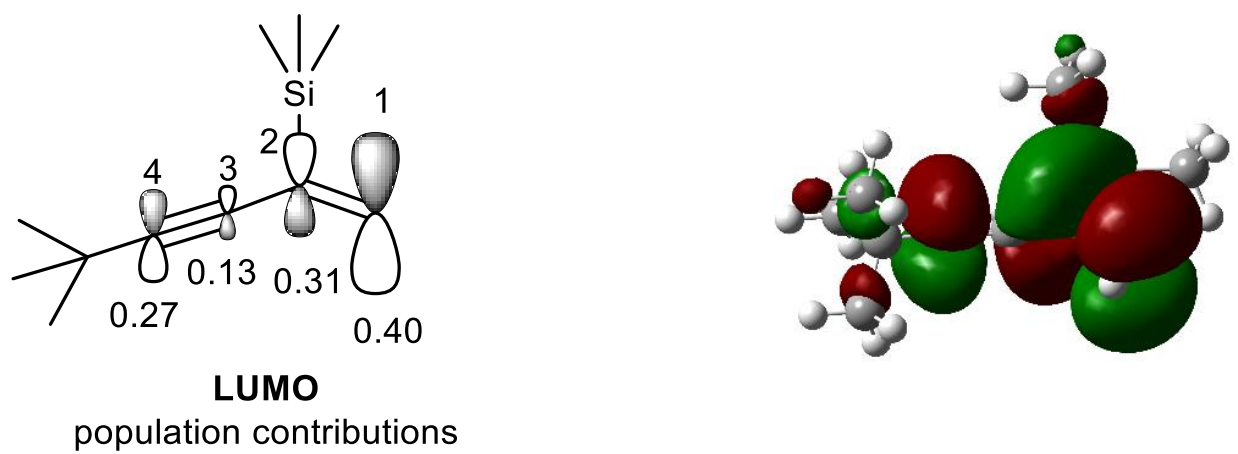

Figure S1. DFT population analysis and isosurface of the LUMO of 2-trimethylsilyl-4-tert-butyl-1butene-3-yne $7 \mathbf{g}$ ). 
(a)

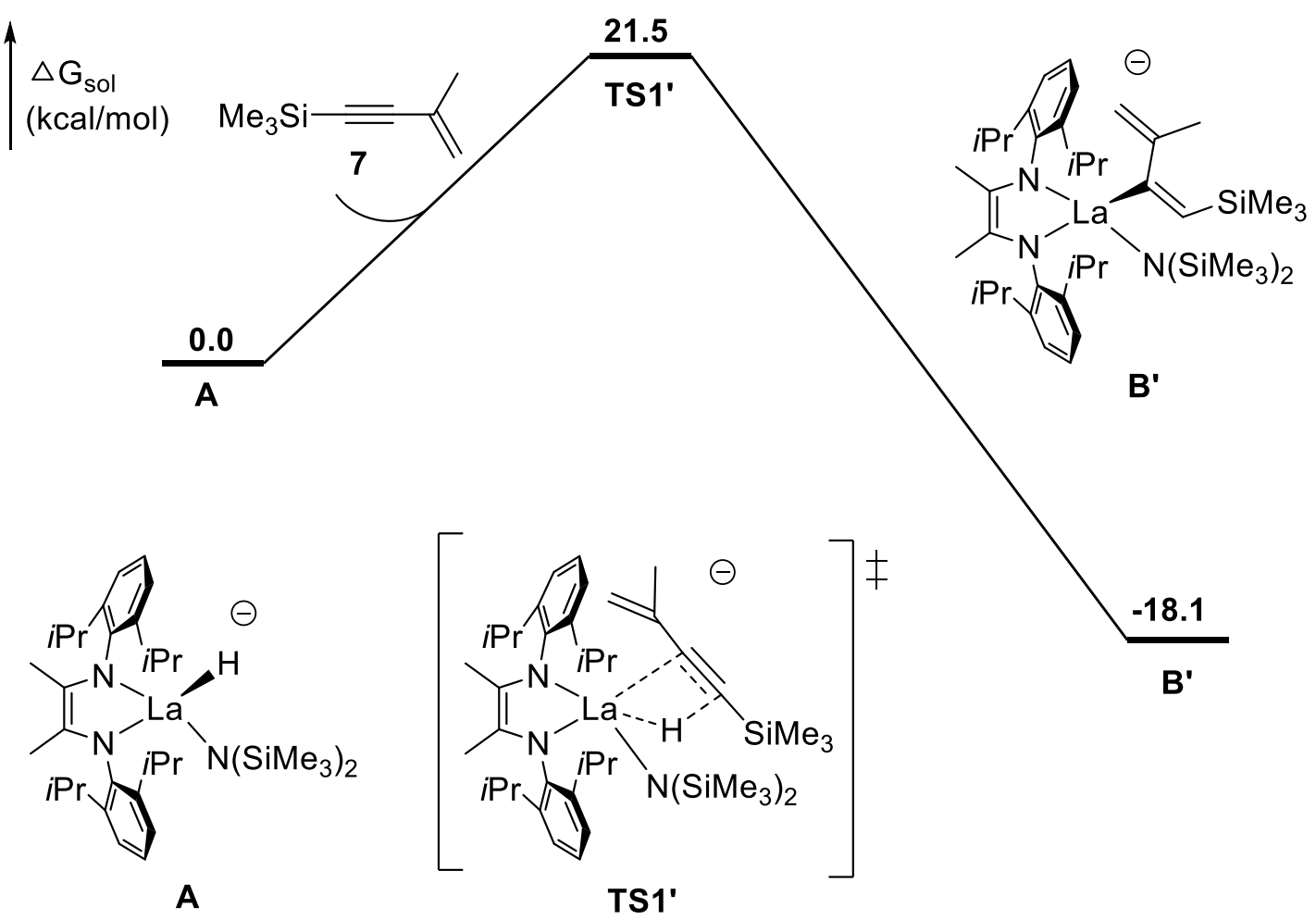

(b)

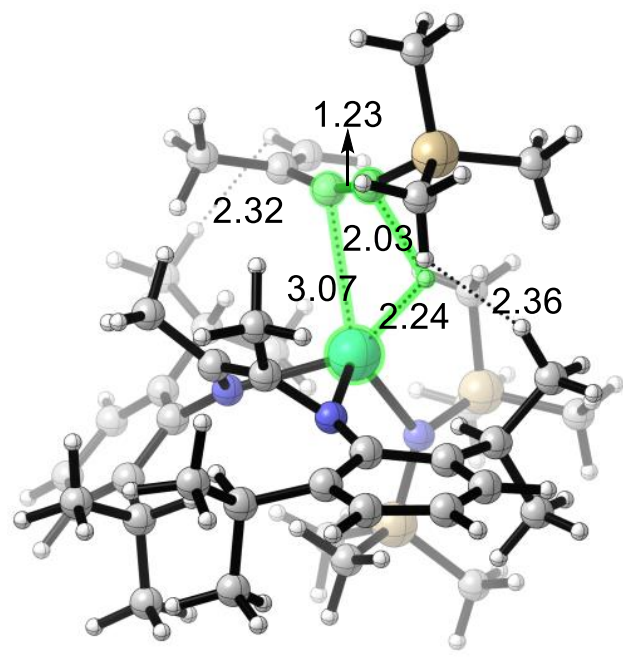

TS1'

Figure S2. (a) Gibbs free-energy profile of the possible 4,3-insertion of 2-methyl-4-trimethylsilyl-1butene-3-yne 7 into lanthanum hydride A. (b) Calculated structure of transition state TS1'. 


\section{The Cartesian Coordinates of the stationary points discussed in the text}

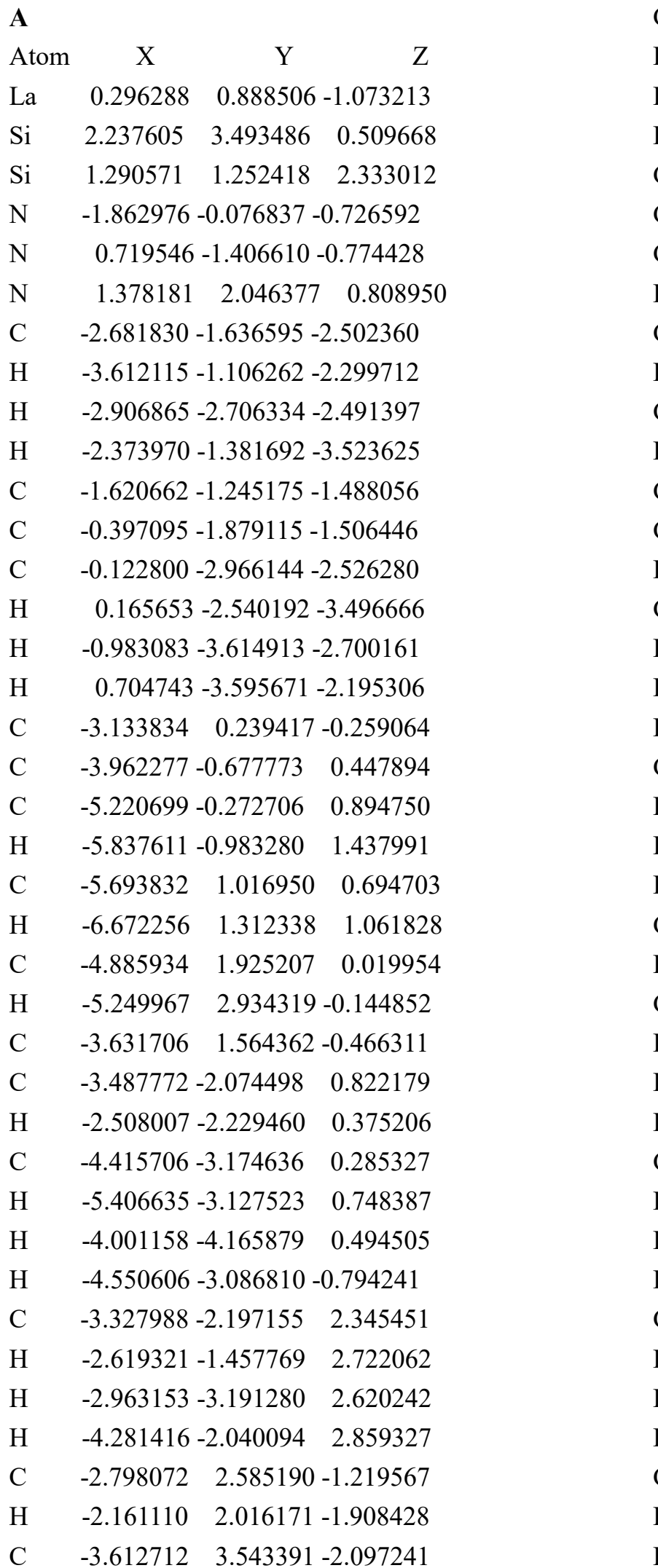

\begin{tabular}{|c|c|c|}
\hline & -4.294464 & $2.995048-2.751621$ \\
\hline & -2.941944 & $4.136794-2.725239$ \\
\hline & -4.206317 & $4.244975-1.503358$ \\
\hline & -1.900726 & $3.388987-0.262617$ \\
\hline & -2.514059 & $4.026428 \quad 0.381432$ \\
\hline & -1.207067 & $4.038324-0.808087$ \\
\hline & -1.314706 & $2.759426 \quad 0.412867$ \\
\hline & $1.722103-$ & $-2.288278-0.353861$ \\
\hline & $1.474705-$ & $-3.273473 \quad 0.635340$ \\
\hline & 2.507894 & $-4.118552 \quad 1.041494$ \\
\hline & $2.310813-$ & $-4.872776 \quad 1.797840$ \\
\hline & $3.790410-$ & $-3.997548 \quad 0.521460$ \\
\hline & $4.586066-$ & $-4.655313 \quad 0.859210$ \\
\hline & $4.045315-$ & $-3.013913-0.426674$ \\
\hline & $5.049003-$ & $-2.912384-0.827615$ \\
\hline & $3.036528-$ & $-2.165348-0.882797$ \\
\hline & $0.112758-$ & $-3.380276 \quad 1.298433$ \\
\hline & $-0.449497-2$ & $2.494738 \quad 1.004155$ \\
\hline & -0.66 & $4.611639 \quad 0.808672$ \\
\hline & $-0.811918-$ & $4.574771-0.271677$ \\
\hline & -1.646856 & $4.670259 \quad 1.282268$ \\
\hline & $-0.121649-$ & $\begin{array}{rl}5.534777 & 1.043365\end{array}$ \\
\hline & $0.226417-$ & $-3.370029 \quad 2.829978$ \\
\hline & $0.715797-$ & $-4.272403 \quad 3.209511$ \\
\hline & $-0.763375-$ & $3.314767 \quad 3.289037$ \\
\hline & $0.804736-$ & $-2.508762 \quad 3.169479$ \\
\hline & $3.351763-$ & $-1.103827-1.921369$ \\
\hline & $2.404300-$ & $-0.839103-2.400560$ \\
\hline & $4.286110-$ & $-1.587543-3.036613$ \\
\hline & $5.300837-$ & $-1.778513-2.673493$ \\
\hline & $4.358958-$ & $-0.827566-3.820082$ \\
\hline & $3.913753-$ & $-2.509304-3.490990$ \\
\hline & 3.916167 & $0.162724-1.257306$ \\
\hline & 3.274017 & $0.529597-0.452494$ \\
\hline & 4.043052 & $0.968218-1.987639$ \\
\hline & $4.891544-$ & $-0.042616-0.804354$ \\
\hline & 2.216718 & $3.882760-1.347010$ \\
\hline & 2.643984 & $3.090863-1.969184$ \\
\hline & 2.799130 & $4.789682-1.543924$ \\
\hline & 1.209810 & $4.070486-1.735529$ \\
\hline & 1.489558 & $5.020788 \quad 1.353756$ \\
\hline & 0.426070 & $5.112458 \quad 1.111253$ \\
\hline & 1.984945 & $5.942798 \quad 1.028014$ \\
\hline
\end{tabular}




$\begin{array}{lccc}\mathrm{H} & 1.570764 & 4.968832 & 2.442486 \\ \mathrm{C} & 4.061312 & 3.413117 & 1.030852 \\ \mathrm{H} & 4.163837 & 3.220618 & 2.103545 \\ \mathrm{H} & 4.591512 & 4.347072 & 0.812647 \\ \mathrm{H} & 4.580146 & 2.604769 & 0.507092 \\ \mathrm{C} & -0.359981 & 0.344347 & 2.501645 \\ \mathrm{H} & -1.205312 & 1.039612 & 2.495672 \\ \mathrm{H} & -0.399887 & -0.214423 & 3.442405 \\ \mathrm{H} & -0.532085 & -0.370722 & 1.693853 \\ \mathrm{C} & 1.393489 & 2.429465 & 3.821904 \\ \mathrm{H} & 2.339320 & 2.978929 & 3.857460 \\ \mathrm{H} & 1.308555 & 1.868000 & 4.759120 \\ \mathrm{H} & 0.585066 & 3.166341 & 3.804510 \\ \mathrm{C} & 2.676756 & -0.017321 & 2.555381 \\ \mathrm{H} & 2.625888 & -0.806880 & 1.800987 \\ \mathrm{H} & 2.635230 & -0.501643 & 3.537658 \\ \mathrm{H} & 3.659344 & 0.456915 & 2.462598 \\ \mathrm{H} & 0.611659 & 1.490899 & -3.221282\end{array}$

2-methyl-4-trimethylsilyl-1-butene-3-yne 7

$\begin{array}{llll}\text { Atom } & \mathrm{X} & \mathrm{Y} & \mathrm{Z}\end{array}$

Si $\quad-1.676699 \quad 0.004455-0.000081$

$\begin{array}{llll}\text { C } & -2.307167 & -0.792788 & 1.582845\end{array}$

H $\quad-1.987491-1.835552 \quad 1.658252$

H $\quad-1.936451-0.2693112 .468132$

H $\quad-3.400987-0.776631 \quad 1.620356$

C $\quad-2.216544 \quad 1.804446-0.083011$

$\begin{array}{llll}\mathrm{H} & -1.842497 & 2.372637 & 0.772921\end{array}$

$\mathrm{H} \quad-1.845906 \quad 2.290565-0.989468$

$\mathrm{H} \quad-3.307984 \quad 1.886495-0.084682$

C $\quad-2.311769-0.936682-1.499925$

H $\quad-1.943539-0.498187-2.431222$

$\mathrm{H} \quad-1.992387-1.982047-1.478635$

$\mathrm{H} \quad-3.405686-0.923753-1.535714$

C $\quad 0.158553-0.058230 \quad 0.000060$

C $\quad 1.372200-0.116586 \quad 0.000151$

C $\quad 3.467616 \quad 1.232349-0.000100$

$\mathrm{H} \quad 4.554559 \quad 1.134306-0.000235$

$\mathrm{H} \quad 3.169545 \quad 1.810821-0.879121$

$\begin{array}{llll}\mathrm{H} & 3.169781 & 1.810903 & 0.878949\end{array}$

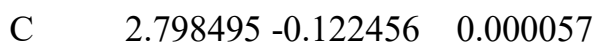

$\begin{array}{llll}\text { C } & 3.486383 & -1.272425 & 0.000134\end{array}$

$\mathrm{H} \quad 2.985191-2.232907 \quad 0.000257$

$\mathrm{H} \quad 4.571043-1.275473 \quad 0.000083$

\section{TS1}

Imaginary frequency: $-49.91 \mathrm{~cm}^{-1}$

$\begin{array}{lccc}\text { Atom } & \mathrm{X} & \mathrm{Y} & \mathrm{Z} \\ \mathrm{La} & -0.242969 & 0.346431 & -1.122185 \\ \mathrm{Si} & -0.481053 & 3.823489 & -2.066425 \\ \mathrm{Si} & -1.872583 & 3.093916 & 0.497004 \\ \mathrm{~N} & -1.514343 & -1.374445 & -0.057434 \\ \mathrm{~N} & 0.719042 & 0.198906 & 1.081920 \\ \mathrm{~N} & -0.938561 & 2.655503 & -0.886362 \\ \mathrm{C} & -0.277429 & -3.463283 & 0.618123 \\ \mathrm{H} & -1.139144 & -3.927336 & 0.140183 \\ \mathrm{H} & -0.210689 & -3.873562 & 1.629741 \\ \mathrm{H} & 0.620954 & -3.790587 & 0.087444 \\ \mathrm{C} & -0.413750 & -1.948852 & 0.625210 \\ \mathrm{C} & 0.624682 & -1.210417 & 1.156635 \\ \mathrm{C} & 1.835564 & -1.958440 & 1.680852 \\ \mathrm{H} & 2.395344 & -2.412420 & 0.858441 \\ \mathrm{H} & 1.570466 & -2.763261 & 2.371122 \\ \mathrm{H} & 2.506522 & -1.282991 & 2.207655\end{array}$

$\mathrm{C}$

C $\quad-3.382610-2.616071 \quad 1.016909$

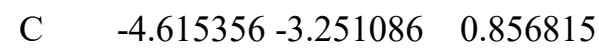

H $\quad-5.080639-3.714161 \quad 1.722170$

C $\quad-5.280664-3.280118-0.360545$

$\mathrm{H} \quad-6.240880-3.777856-0.457090$

C $\quad-4.700588-2.638941-1.449562$

$\mathrm{H} \quad-5.220345-2.641538-2.401849$

C $\quad-3.466531-2.003302-1.349461$

C $\quad-2.824505-2.509582 \quad 2.430222$

H $\quad-1.820555-2.094183 \quad 2.374139$

C $\quad-2.725110-3.866390 \quad 3.144554$

$\mathrm{H} \quad-3.713610-4.281830 \quad 3.364001$

$\mathrm{H} \quad-2.198935-3.758893 \quad 4.098208$

$\begin{array}{llll}\mathrm{H} & -2.185856 & -4.599069 & 2.541697\end{array}$

C $\quad-3.675131-1.534362 \quad 3.259584$

$\begin{array}{llll}\mathrm{H} & -3.690522 & -0.544741 & 2.801717\end{array}$

$\mathrm{H} \quad-3.276416-1.435432 \quad 4.273807$

H $\quad-4.708842-1.885258 \quad 3.338776$

C $\quad-2.892586-1.284948-2.554607$

H $\quad-1.802399-1.381158-2.495125$

C $\quad-3.274197-1.883484-3.911946$

$\mathrm{H} \quad-3.073186-2.957173-3.943678$

$\mathrm{H} \quad-2.691860-1.400532-4.701411$ 
$-4.331295-1.730940-4.148782$

$\begin{array}{ll}-3.249311 & 0.210804-2.526918\end{array}$

$-4.318748 \quad 0.345720-2.713621$

$-2.691566 \quad 0.767891-3.284768$

$-3.059753 \quad 0.671934-1.553885$

$\begin{array}{llll}1.373573 & 0.887826 & 2.101178\end{array}$

$\begin{array}{lll}1.057942 & 0.694424 & 3.473626\end{array}$

$\begin{array}{llll}1.835274 & 1.309134 & 4.456844\end{array}$

$\begin{array}{llll}1.597554 & 1.134017 & 5.502707\end{array}$

$\begin{array}{llll}2.874402 & 2.167928 & 4.131186\end{array}$

$\begin{array}{llll}3.470825 & 2.638048 & 4.907374\end{array}$

$\begin{array}{llll}3.103240 & 2.460385 & 2.788992\end{array}$

$\begin{array}{llll}3.872898 & 3.180143 & 2.534538\end{array}$

$\begin{array}{lll}2.368334 & 1.855583 & 1.772779\end{array}$

$-0.195598-0.054362 \quad 3.901583$

$\begin{array}{lll}-0.692488 & -0.406139 & 2.999925\end{array}$

$0.093759-1.277422 \quad 4.781398$

$0.754261-1.982764 \quad 4.275935$

$-0.833088-1.802915 \quad 5.031936$

$0.572844-0.987528 \quad 5.722292$

$\begin{array}{lll}-1.159433 & 0.910765 & 4.613125\end{array}$

$\begin{array}{lll}-0.734935 & 1.276557 & 5.553096\end{array}$

$\begin{array}{llll}-2.107727 & 0.417874 & 4.842500\end{array}$

$\begin{array}{lll}-1.370835 & 1.777750 & 3.985862\end{array}$

$\begin{array}{lll}2.611528 & 2.229149 & 0.317972\end{array}$

$1.621877 \quad 2.305955-0.143922$

$3.426033 \quad 1.156352-0.420832$

$4.449031 \quad 1.129272-0.032991$

$3.485383 \quad 1.364755-1.494664$

$3.000123 \quad 0.161582-0.289964$

$\begin{array}{llll}3.262449 & 3.603361 & 0.133481\end{array}$

$\begin{array}{llll}2.715056 & 4.378818 & 0.674027\end{array}$

$3.276247 \quad 3.877162-0.924037$

$\begin{array}{llll}4.299968 & 3.614655 & 0.481615\end{array}$

$1.0409893 .274223-3.051131$

$1.918972 \quad 3.128398-2.416444$

$1.303460 \quad 4.039854-3.790122$

$0.852835 \quad 2.340356-3.588419$

$-1.849331 \quad 4.129695-3.342095$

$-2.083706 \quad 3.201776-3.872742$

$\begin{array}{lll}-1.555578 & 4.874873-4.090278\end{array}$

$-2.770965 \quad 4.477700-2.867167$

$-0.015254 \quad 5.513300-1.331062$

$-0.840254 \quad 5.990561-0.795651$

\section{B}

$\begin{array}{lrrr}\text { Atom } & \text { X } & \text { Y } & \text { Z } \\ \text { La } & -0.495116 & -0.535459 & 0.104616 \\ \text { Si } & -3.250351 & -0.621656 & 2.353939 \\ \text { Si } & -1.212063 & 1.477104 & 3.098937\end{array}$

\section{$0.300523 \quad 6.200778-2.124040$}

$0.817681 \quad 5.416908-0.629069$

$\begin{array}{llll}-2.689491 & 1.582636 & 1.286040\end{array}$

$\begin{array}{llll}-3.433582 & 1.112692 & 0.636716\end{array}$

$\begin{array}{lll}-3.209216 & 1.889274 & 2.199849\end{array}$

$\begin{array}{llll}-1.968947 & 0.812225 & 1.562407\end{array}$

$\begin{array}{llll}-3.313121 & 4.262915 & 0.076088\end{array}$

$\begin{array}{llll}-2.983027 & 5.222121 & -0.332417\end{array}$

$\begin{array}{llll}-3.910573 & 4.477666 & 0.969388\end{array}$

$-3.979210 \quad 3.805413-0.662331$

$\begin{array}{llll}-0.842246 & 3.931934 & 1.843969\end{array}$

$\begin{array}{lll}-0.029362 & 3.281844 & 2.176648\end{array}$

$\begin{array}{llll}-1.450242 & 4.178469 & 2.721965\end{array}$

$\begin{array}{llll}-0.391148 & 4.861180 & 1.485212\end{array}$

$1.196921-2.359854-2.969386$

$1.058516-1.386305-3.910423$

$2.357928-2.381555-2.156695$

$3.393021-2.400908-1.509242$

$5.108033-2.455119-0.891186$

$5.783578-4.198953-1.155126$

$6.122247-1.227847-1.902086$

$5.261545-2.028018 \quad 0.934965$

$5.195284-4.935427-0.600228$

$5.751157-4.480185-2.211290$

$6.821981-4.278379-0.816096$

$6.087324-1.472777-2.967212$

$5.736746-0.212231-1.784747$

$7.171891-1.226631-1.589838$

$4.833978-1.051069 \quad 1.166543$

$\begin{array}{lll}4.757535 & -2.761714 & 1.568198\end{array}$

$\begin{array}{lll}6.318147 & -2.008785 & 1.224078\end{array}$

$\begin{array}{llll}-0.047667 & 0.387532 & -3.416532\end{array}$

$0.156077-3.446173-2.800345$

$-0.490413-3.270313-1.939839$

$0.625568-4.423131-2.656778$

$-0.480470-3.498317-3.686174$

$0.257024-1.423110-4.633785$

$1.841046-0.665278-4.097866$

$\begin{array}{llll}\text { Si } & -1.212063 & 1.477104 & 3.098937\end{array}$ 


\begin{tabular}{|c|c|c|c|}
\hline & 1.91 & 0 & \\
\hline & 0.207135 & 1.552437 & -0.763655 \\
\hline & -1.785302 & 0.218999 & 2.070543 \\
\hline & 2.441281 & $1.002065-2$ & 2.476612 \\
\hline & 3.046983 & $1.811252-2$ & 2.072068 \\
\hline & 3.075808 & $0.424746-3$ & 3.153614 \\
\hline & 1.647579 & $1.450230-3$ & 3.084758 \\
\hline & 1.853040 & $0.163091-1$ & 1.353544 \\
\hline & 1.070798 & 0.926949 & -1.685317 \\
\hline & 0.917291 & 1.319103 & -3.142387 \\
\hline & 0.244005 & 0.635504 & -3.671423 \\
\hline & 1.865234 & 1.317378 & -3.684622 \\
\hline & 0.491341 & 2.320013 & -3.217660 \\
\hline & 3.047312 & 1.332458 & 0.426369 \\
\hline & 4.382976 & 0.997855 & 0.041421 \\
\hline & 5.445244 & 1.811638 & 0.440960 \\
\hline & 6.450152 & 1.552101 & 0.120608 \\
\hline & 5.265839 & 2.910669 & 1.266132 \\
\hline & 6.105773 & -3.534058 & 1.557168 \\
\hline & 3.987350 & 3.164630 & 1.755549 \\
\hline & 3.847320 & 3.986938 & 2.447551 \\
\hline & 2.890961 & 2.397874 & 1.378020 \\
\hline & 4.733393 & 0.320844 & -0.638305 \\
\hline & 3.820346 & 0.776208 & -1.015251 \\
\hline & 5.697912 & 0.177436 & -1.824571 \\
\hline & 6.697544 & $0.131811-1$ & 1.503838 \\
\hline & 5.807509 & 1.134700 & -2.344243 \\
\hline & 5.339273 & $0.559263-2$ & 2.545562 \\
\hline & 5.318251 & 1.280175 & 0.412062 \\
\hline & 4.607053 & 1.444905 & 1.223997 \\
\hline & 5.562709 & 2.250788 & -0.030251 \\
\hline & 6.233372 & 0.868843 & 0.848975 \\
\hline & 1.537223 & 2.646225 & 2.017412 \\
\hline & 0.794655 & 2.657135 & 1.206061 \\
\hline & 1.389901 & 3.985284 & 2.745082 \\
\hline & 1.617042 & 4.836178 & 2.098978 \\
\hline & 0.364967 & -4.098828 & 3.107673 \\
\hline & 2.045247 & 4.039125 & 3.619233 \\
\hline & 1.202938 & 1.506839 & 2.992918 \\
\hline & 1.863613 & 1.561320 & 3.863480 \\
\hline & 0.169252 & 1.552716 & 3.343467 \\
\hline & 1.368773 & 0.534910 & 2.532920 \\
\hline & -0.045669 & $2.925733-$ & -0.807329 \\
\hline & 1.004258 & 3.880417 & -0.775102 \\
\hline
\end{tabular}

\begin{tabular}{|c|c|c|c|}
\hline & & & \\
\hline & 517008 & 59 & \\
\hline 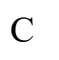 & 603800 & 5.6951 & 33 \\
\hline & 819113 & 6.7575 & \\
\hline & 59 & 4.7 & \\
\hline & 95 & 5.12 & \\
\hline & 29 & 3.39 & \\
\hline & & 3.4 & \\
\hline & & & \\
\hline & 64 & 3.68 & 704 \\
\hline & 2.912469 & 3.136 & 400 \\
\hline & & & \\
\hline & 256 & $4.74^{\prime}$ & 1864 \\
\hline & 3.060155 & 4.199619 & 0.65093 \\
\hline & 294 & 5.279 & 0.4 \\
\hline & & 3.83 & \\
\hline & 54 & 4.03422 & 629 \\
\hline & -2.554293 & 2.415304 & .823629 \\
\hline & -2.31 & 1.6285 & 62 \\
\hline & & 1.7 & 21 \\
\hline $\mathrm{H}$ & -2.968068 & 2.5519 & .948932 \\
\hline $\mathrm{H}$ & -3.507901 & 1.033517 & -2.214151 \\
\hline 11 & -1.79 & 1.296 & .531004 \\
\hline & 3.895408 & 2.9997 & -0.36 \\
\hline $\mathrm{H}$ & -3.815416 & 3.509952 & $0.59220^{\circ}$ \\
\hline $\mathrm{H}$ & -4.626229 & 2.193235 & -0.258136 \\
\hline $\mathrm{H}$ & 4.303862 & 3.706000 & -1.098253 \\
\hline & -3.99 & 1.230771 & 0.718411 \\
\hline $\mathrm{H}$ & $-4.264380-$ & 0.390781 & 0.07 \\
\hline $\mathrm{H}$ & -4.9149 & 63 & \\
\hline 11 & $-3.351385-$ & 1.885751 & \\
\hline $\mathrm{C}$ & $-2.978334-$ & 2.161351 & 3.4 \\
\hline $\mathrm{H}$ & $-2.261344-$ & 2.844570 & 2974 \\
\hline $\mathrm{H}$ & $-3.902521-$ & 2.724934 & 3.601872 \\
\hline $\mathrm{H}$ & $-2.569126-$ & 1.887753 & 4.409106 \\
\hline $\mathrm{C}$ & -4.632291 & 0.391885 & 3.168030 \\
\hline $\mathrm{H}$ & -4.347033 & 0.791895 & 4.144689 \\
\hline 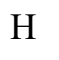 & $-5.525596-$ & 0.225530 & 3.314653 \\
\hline $\mathrm{H}$ & -4.917447 & 1.239313 & 2.53783 \\
\hline $\mathrm{C}$ & 0.570284 & 1.995802 & 2.71299 \\
\hline $\mathrm{H}$ & 1.311931 & 1.422230 & 3.27293 \\
\hline & 0.696534 & 3.046448 & 2.99402 \\
\hline $\mathrm{H}$ & 0.820574 & 1.924177 & 1.65198 \\
\hline$r$ & 1.232635 & 0.973899 & 4.933412 \\
\hline
\end{tabular}




\begin{tabular}{|c|c|c|}
\hline-2.242311 & 0750 & \\
\hline-0.818589 & 1.767448 & \\
\hline 9998 & 5281 & \\
\hline-2.212081 & 3.080670 & \\
\hline-2.026054 & 3.557731 & \\
\hline-1.908774 & 3.793155 & \\
\hline-3.288511 & 2.922378 & \\
\hline \multicolumn{3}{|c|}{$-0.549565-3.807781-0.717552$} \\
\hline \multicolumn{3}{|c|}{$\begin{array}{lll}-1.344550 & -4.627434 & 0.27\end{array}$} \\
\hline \multicolumn{3}{|c|}{$-1.132107-2.832835-1.421924$} \\
\hline \multicolumn{3}{|c|}{$-1.640326-1.783988-1.965254$} \\
\hline \multicolumn{3}{|c|}{$-2.662350-1.957057-3.493778$} \\
\hline \multicolumn{3}{|c|}{$-2.499756-3.696830-4.229033$} \\
\hline \multicolumn{3}{|c|}{$-4.490516-1.692173-3.084444$} \\
\hline \multicolumn{3}{|c|}{$-2.146315-0.737257-4.841448$} \\
\hline \multicolumn{3}{|c|}{$-1.464040-3.905105-4.514388$} \\
\hline \multicolumn{3}{|c|}{$-2.790202-4.456993-3.497663$} \\
\hline \multicolumn{3}{|c|}{$-3.125217-3.828808-5.118792$} \\
\hline \multicolumn{3}{|c|}{$-4.827206-2.418174-2.338794$} \\
\hline \multicolumn{3}{|c|}{$-4.681152-0.698167-2.674536$} \\
\hline \multicolumn{3}{|c|}{$-5.116206-1.811593-3.975685$} \\
\hline-2.283866 & $0.301280-4$ & 4.535414 \\
\hline-1.091634 & $-0.866585-5$. & .102370 \\
\hline \multicolumn{3}{|c|}{$-2.732414-0.897733-5.752955$} \\
\hline \multicolumn{3}{|c|}{$-0.817193-4.737710 \quad 1$} \\
\hline \multicolumn{3}{|c|}{$0.826667-4.319382-1.079961$} \\
\hline \multicolumn{3}{|c|}{$1.487038-4.385698-0.210251$} \\
\hline \multicolumn{3}{|c|}{$1.313689-3.669123-1.806368$} \\
\hline \multicolumn{3}{|c|}{$0.769038-5.328051-1.514572$} \\
\hline \multicolumn{3}{|c|}{$-1.520868-5.645857-0.100881$} \\
\hline$-2.31 \mathrm{c}$ & 802 & 0.48 \\
\hline
\end{tabular}

TS1'

Imaginary frequency: $-146.22 \mathrm{~cm}^{-1}$

$\begin{array}{lccc}\text { Atom } & \mathrm{X} & \mathrm{Y} & \mathrm{Z} \\ \mathrm{La} & 0.354375 & -0.730687 & 0.023281 \\ \mathrm{Si} & 0.562616 & -2.784874 & 2.831503 \\ \mathrm{Si} & -1.165383 & -0.312664 & 3.307847 \\ \mathrm{~N} & 0.750950 & 1.649128 & -0.181299 \\ \mathrm{~N} & -1.657907 & 0.192233 & -0.902259 \\ \mathrm{~N} & -0.121284 & -1.295816 & 2.356003 \\ \mathrm{C} & 1.128945 & 2.100151 & -2.635932 \\ \mathrm{H} & 2.050333 & 2.533607 & -2.253423 \\ \mathrm{H} & 1.038681 & 5.025303 & -2.782317\end{array}$

$1.401399 \quad 1.268932-3.293520$

$0.242313 \quad 1.621116-1.496949$

$-0.939619 \quad 0.980396-1.822843$

$\begin{array}{lll}-1.363082 & 0.885984-3.276147\end{array}$

$-0.757366 \quad 0.158997-3.831949$

$-1.276939 \quad 1.836561-3.808268$

$-2.403503 \quad 0.562248-3.336560$ $\begin{array}{lll}1.281014 & -3.637812 & 1.286371\end{array}$ $\begin{array}{lll}0.508777-3.898415 & 0.554588\end{array}$ $\begin{array}{lll}1.761853-4.579385 & 1.570608\end{array}$ $\begin{array}{lll}2.054411 & -3.055492 & 0.768852\end{array}$ $2.001286-2.633463 \quad 4.054597$ $2.811818-2.035152 \quad 3.628433$ $2.415910-3.613437 \quad 4.317046$ $1.689879-2.145142 \quad 4.982152$ $-0.680111-4.002865 \quad 3.585156$ $-1.106580-3.613980 \quad 4.515273$ $\begin{array}{lll}-0.215438-4.967909 & 3.816309\end{array}$ $-1.512344-4.186818 \quad 2.899852$ $\begin{array}{lll}-1.219763 & 1.454262 & 2.648697\end{array}$ $\begin{array}{lll}-0.251515 & 1.957149 & 2.669099\end{array}$ $\begin{array}{lll}-1.919962 & 2.053456 & 3.240962\end{array}$ $\begin{array}{lll}-1.562630 & 1.479853 & 1.612239\end{array}$ $-0.643759-0.243075 \quad 5.133068$ $-0.690438-1.228645 \quad 5.608344$ $\begin{array}{lll}-1.299835 & 0.423413 & 5.703894\end{array}$ $\begin{array}{llll}0.379360 & 0.126058 & 5.242090\end{array}$ $-2.962202-0.919249 \quad 3.286293$ $\begin{array}{lll}-3.404108 & -0.812163 & 2.291351\end{array}$ $\begin{array}{lll}-3.581581 & -0.339226 & 3.980070\end{array}$ $-3.043810-1.972720 \quad 3.570669$ $-0.292504-4.691878-2.249472$ $-1.062643-5.338452-2.653453$ $-0.076158-3.469240-2.768058$ $0.982404-2.635717-2.306546$ $-0.890871-2.928964-3.920463$ $2.089616-2.095637-2.221851$ $-0.238538-2.698950-4.767912$ $-1.643098-3.650840-4.240434$ $-1.398183-2.002443-3.646569$ $3.872301-1.689255-2.365523$ $4.448207-2.522879-3.977101$ $4.230733 \quad 0.144588-2.554038$ $4.843386-2.500568-0.970864$ 
C

C $\quad-3.831462 \quad 1.351070-0.730484$

C $\quad-3.738539-1.084426-0.892863$

C $\quad-5.223454 \quad 1.264917-0.689766$
C $\quad-5.129718-1.117301-0.801485$

C $\quad-2.980789-2.391502-1.040976$

$\mathrm{H} \quad-5.804764 \quad 2.177564-0.600851$

$\begin{array}{llll}\text { C } & -5.884293 & 0.045179 & -0.718357\end{array}$

$\mathrm{H} \quad-2.130523 \quad 2.564123-0.362471$

C $\quad-3.330350 \quad 3.578729-1.817733$

$\begin{array}{llll}\text { C } & -3.775445 & 3.454028 & 0.666713\end{array}$

$\mathrm{H} \quad-5.639207-2.075001-0.814668$

$\mathrm{H} \quad-2.030301-2.143493-1.517826$

C $\quad-3.698152-3.403638-1.943118$

C $\quad-2.659158-3.039162 \quad 0.313580$

H $\quad-6.968039-0.000322-0.664780$

$\mathrm{H} \quad-2.871041 \quad 3.091306-2.678263$

$\mathrm{H} \quad-2.853748 \quad 4.554365-1.688639$

$\mathrm{H} \quad-4.385354 \quad 3.750873-2.055952$

$\mathrm{H} \quad \begin{array}{llll}4.823015 & 3.729657 & 0.513605\end{array}$

$\begin{array}{llll}\mathrm{H} & -3.221831 & 4.373310 & 0.864471\end{array}$

$\mathrm{H} \quad-3.722143 \quad 2.829020 \quad 1.559673$

$\mathrm{H} \quad-4.608497-3.792059-1.477454$

$\mathrm{H} \quad-3.047434-4.258211-2.135808$

$\mathrm{H} \quad-3.978170-2.958115-2.900875$

$\mathrm{H} \quad-2.099266-2.373768 \quad 0.972401$

$\mathrm{H} \quad-2.070930-3.952018 \quad 0.173594$

$\mathrm{H} \quad-3.578654-3.304550 \quad 0.844575$

\section{B' $^{\prime}$}

$\begin{array}{lccc}\text { Atom } & \mathrm{X} & \mathrm{Y} & \mathrm{Z} \\ \mathrm{La} & 0.566707 & -0.412087 & 0.242468 \\ \mathrm{Si} & 1.138884 & -2.504256 & 3.047990 \\ \mathrm{Si} & -1.730961 & -1.486995 & 2.803722 \\ \mathrm{~N} & -0.355795 & 1.805385 & 0.021285 \\ \mathrm{~N} & -1.240550 & -0.627701 & -1.239054 \\ \mathrm{~N} & -0.082779 & -1.564575 & 2.315374 \\ \mathrm{C} & 0.302805 & 2.707180 & -2.231712 \\ \mathrm{H} & 0.631541 & 3.540875 & -1.612554 \\ \mathrm{H} & -1.430795 & 5.115704 & -2.450863 \\ \mathrm{H} & 1.188396 & 2.279429 & -2.712900 \\ \mathrm{C} & -0.411595 & 1.667001 & -1.383314 \\ \mathrm{C} & -0.861533 & 0.507570 & -1.983594 \\ \mathrm{C} & -0.702847 & 0.315029 & -3.477531 \\ \mathrm{H} & 0.313022 & -0.017744 & -3.726344 \\ \mathrm{H} & -0.897194 & 1.225046 & -4.048345 \\ \mathrm{H} & -1.390871 & -0.455491 & -3.829973\end{array}$




\begin{tabular}{|c|c|c|c|c|c|c|}
\hline$y$ & $2.701935-2.446210$ & 1.965473 & $\mathrm{H}$ & \multicolumn{3}{|c|}{$6.251435-0.197381-0.457791$} \\
\hline $\mathrm{H}$ & $2.560983-2.833420$ & 0.951680 & $\mathrm{H}$ & 6.976270 & 1.408112 & -0.652581 \\
\hline & $3.486751-3.061262$ & 2.418283 & $\mathrm{H}$ & \multicolumn{3}{|c|}{$4.712832-2.391526-0.372912$} \\
\hline & $3.129491-1.440771$ & 1.869911 & $\mathrm{H}$ & 2.378366 & 1.205083 & -0.633588 \\
\hline & $1.689806-1.879909$ & 4.752609 & $\mathrm{C}$ & -0.503587 & 3.021394 & 0.657529 \\
\hline & $2.067855-0.854799$ & 4.690329 & $\mathrm{C}$ & 0.127476 & 3.211588 & 1.930293 \\
\hline & $2.491629-2.501034$ & 5.168004 & $\mathrm{C}$ & -1.286893 & 4.108825 & 0.156179 \\
\hline & $0.865776-1.878406$ & 5.470970 & $\mathrm{C}$ & 0.162084 & 4.473297 & 2.512249 \\
\hline & $0.683982-4.333656$ & 3.258133 & $\mathrm{C}$ & 0.759922 & 2.041585 & 2.666204 \\
\hline 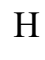 & $-0.185779-4.454527$ & 3.911966 & $\mathrm{C}$ & -1.205199 & 5.359052 & 0.772092 \\
\hline & $1.505555-4.911989$ & 3.695304 & $\mathrm{C}$ & -2.372921 & \multicolumn{2}{|c|}{$3.905564-0.894018$} \\
\hline & $0.430669-4.787960$ & 2.296214 & $\mathrm{H}$ & 0.680289 & 4.603409 & 3.457400 \\
\hline & $\begin{array}{ll}-2.523820 & 0.144035\end{array}$ & 2.269807 & $\mathrm{C}$ & -0.455043 & 5.572014 & 1.920242 \\
\hline & $-2.089764 \quad 1.008793$ & 2.778179 & $\mathrm{H}$ & 0.264496 & 1.132883 & 2.319007 \\
\hline & $\begin{array}{ll}-3.594394 & 0.132217\end{array}$ & 2.501614 & $\mathrm{C}$ & 2.269007 & 1.927434 & 2.395528 \\
\hline & $-2.427020 \quad 0.323608$ & 1.196335 & $\mathrm{C}$ & 0.493754 & 2.050330 & 4.177468 \\
\hline & $-1.954874-1.609930$ & 4.686129 & $\mathrm{H}$ & -1.782375 & 6.182277 & 0.360731 \\
\hline & $-1.590774-2.561846$ & 5.085927 & $\mathrm{H}$ & -2.212644 & 2.949099 & -1.387872 \\
\hline & $-3.012052-1.524491$ & 4.960820 & $\mathrm{C}$ & -2.408807 & 4.986311 & -1.983353 \\
\hline $\mathrm{H}$ & $-1.414374-0.809124$ & 5.199869 & $\mathrm{C}$ & -3.732177 & 3.819241 & -0.177650 \\
\hline & $-2.767754-2.869069$ & 2.027474 & $\mathrm{H}$ & -0.399461 & 6.555412 & 2.376860 \\
\hline 1 & $-2.803695-2.773922$ & 0.938154 & $\mathrm{H}$ & 2.495751 & 1.950420 & 1.328358 \\
\hline $\mathrm{H}$ & $-3.802246-2.849127$ & 2.388798 & $\mathrm{H}$ & 2.687646 & 1.007813 & 2.819778 \\
\hline $\mathrm{H}$ & $-2.355791-3.857266$ & 2.255296 & $\mathrm{H}$ & 2.796399 & 2.771562 & 2.849383 \\
\hline$C$ & \multicolumn{2}{|c|}{$4.395252-2.554619-1.396754$} & $\mathrm{H}$ & 1.039613 & 2.848579 & 4.688425 \\
\hline $\mathrm{H}$ & \multicolumn{2}{|c|}{$4.873210-3.364924-1.939883$} & $\mathrm{H}$ & 0.807881 & 1.100605 & 4.617170 \\
\hline & \multicolumn{2}{|c|}{$3.418560-1.817398-1.955331$} & $\mathrm{H}$ & -0.569727 & 2.184176 & 4.386444 \\
\hline (c) & \multicolumn{2}{|c|}{$2.653964-0.789803-1.262360$} & $\mathrm{H}$ & -2.717750 & 5.958029 & -1.585768 \\
\hline C & \multicolumn{2}{|c|}{$2.974358-2.063463-3.379871$} & $\mathrm{H}$ & -3.125752 & 4.715715 & -2.765269 \\
\hline & \multicolumn{2}{|c|}{$3.060466 \quad 0.487925-1.133844$} & $\mathrm{H}$ & -0.328482 & 3.117180 & -3.024675 \\
\hline & \multicolumn{2}{|c|}{$3.158251-1.175008-3.991979$} & $\mathrm{H}$ & -3.730106 & 3.010115 & 0.555476 \\
\hline H & \multicolumn{2}{|c|}{$3.501839-2.908881-3.829207$} & $\mathrm{H}$ & -4.545616 & 3.637370 & -0.887165 \\
\hline$\Pi$ & \multicolumn{2}{|c|}{$1.900735-2.256429-3.428312$} & $\mathrm{H}$ & -3.950323 & 4.749920 & 0.355179 \\
\hline & \multicolumn{2}{|c|}{$4.663277 \quad 1.313865-1.637558$} & $\mathrm{C}$ & \multicolumn{3}{|c|}{$-2.224216-1.514656-1.686928$} \\
\hline $\mathrm{C}$ & \multicolumn{2}{|c|}{$5.223775 \quad 0.821880-3.372841$} & $\mathrm{C}$ & \multicolumn{3}{|c|}{$-3.578822-1.111733-1.806748$} \\
\hline & \multicolumn{2}{|c|}{$4.384434 \quad 3.184041-1.582456$} & $\mathrm{C}$ & \multicolumn{3}{|c|}{$-1.882976-2.867309-1.964270$} \\
\hline & \multicolumn{2}{|c|}{$6.046222 \quad 0.875733-0.423856$} & $\mathrm{C}$ & \multicolumn{3}{|c|}{$-4.541496-2.043377-2.196097$} \\
\hline H & \multicolumn{2}{|c|}{$5.424009-0.250554-3.425601$} & $\mathrm{C}$ & -3.998649 & 0.313849 & -1.488387 \\
\hline $\mathrm{H}$ & \multicolumn{2}{|c|}{$4.462885 \quad 1.059870-4.121530$} & $\mathrm{C}$ & \multicolumn{3}{|c|}{$-2.885198-3.769742-2.321133$} \\
\hline & $6.142064 \quad 1.350215$ & -3.651708 & $\mathrm{C}$ & \multicolumn{3}{|c|}{$-0.440120-3.334154-1.876445$} \\
\hline & $3.612601 \quad 3.491614$ & -2.294285 & $\mathrm{H}$ & -5.577259 & $1.729982-$ & 2.286518 \\
\hline & $4.049823 \quad 3.506335$ & -0.591122 & $\mathrm{C}$ & -4.209912 & $3.369437-2$ & 2.445378 \\
\hline & $5.296989 \quad 3.741302$ & -1.818645 & $\mathrm{H}$ & -3.162216 & 0.788155 & -0.975032 \\
\hline & $5.763117 \quad 1.124810$ & 0.603428 & $\mathrm{C}$ & -4.272043 & 1.108527 & -2.774728 \\
\hline
\end{tabular}




\begin{tabular}{|c|c|}
\hline & -5.207921 \\
\hline & $-2.624300-4.804456$ \\
\hline & $0.181601-2.43$ \\
\hline & -0.036 \\
\hline & $-0.127893-3.969217-($ \\
\hline & $-4.976210-4.084419-2.7303$ \\
\hline & $-3.389604 \quad 1.131029-3.416$ \\
\hline & $-4.550257 \quad 2.141685-2.5$ \\
\hline & $-5.090045 \quad 0.656712-3.34$ \\
\hline & $-6.114774-0.026080-1.0173$ \\
\hline & $-5.413353 \quad 1.390825-0.23$ \\
\hline & $\begin{array}{lll}-5.021312 & -0.227342 \quad 0.35\end{array}$ \\
\hline & $-0.548774-5.252064-2.9348$ \\
\hline & $1.037866-4.489293-2.963$ \\
\hline & $-0.263389-3.861691-3.987 t$ \\
\hline & $-0.389478-3.314364 \quad 0.321$ \\
\hline & $0.935278-4.217273-0.432$ \\
\hline & -0.70310 \\
\hline
\end{tabular}

$\mathrm{MeSiH}_{3} 6$

$\begin{array}{lccc}\text { Atom } & \mathrm{X} & \mathrm{Y} & \mathrm{Z} \\ \mathrm{Si} & -0.000000 & 0.000000 & 0.637585 \\ \mathrm{H} & -0.000000 & 1.392204 & 1.166676 \\ \mathrm{H} & 1.205684 & -0.696102 & 1.166676 \\ \mathrm{C} & 0.000000 & -0.000000 & -1.250184 \\ \mathrm{H} & -0.886143 & 0.511615 & -1.641704 \\ \mathrm{H} & 0.886143 & 0.511615 & -1.641704 \\ \mathrm{H} & -0.000000 & -1.023230 & -1.641704 \\ \mathrm{H} & -1.205684 & -0.696102 & 1.166676\end{array}$

\section{B-6}

\begin{tabular}{lccc} 
Atom & $\mathrm{X}$ & $\mathrm{Y}$ & $\mathrm{Z}$ \\
$\mathrm{La}$ & -0.347075 & -0.482259 & -0.335570 \\
$\mathrm{Si}$ & -1.321412 & -3.118099 & 2.076465 \\
$\mathrm{Si}$ & 0.849948 & -1.207677 & 2.890954 \\
$\mathrm{~N}$ & \multicolumn{4}{c}{1.997399} & -0.338622 & -0.964886 \\
$\mathrm{~N}$ & 0.046290 & 1.868326 & -0.649765 \\
$\mathrm{~N}$ & -0.279815 & -1.793499 & 1.730276 \\
$\mathrm{C}$ & 2.684639 & 0.658516 & -3.150251 \\
$\mathrm{H}$ & 3.394178 & -0.167311 & -3.097805 \\
$\mathrm{H}$ & 3.252317 & 1.565935 & -3.373132 \\
$\mathrm{H}$ & 2.033504 & 0.478975 & -4.014238 \\
$\mathrm{C}$ & 1.895485 & 0.755952 & -1.855764 \\
$\mathrm{C}$ & 1.000101 & 1.793556 & -1.701527
\end{tabular}

$0.912118 \quad 2.827447-2.812929$

$$
\begin{array}{lll}
0.488923 & 2.418040-3.738780
\end{array}
$$

$\begin{array}{lll}1.892994 & 3.230499-3.078428\end{array}$

$0.287198 \quad 3.667472-2.512490$

$3.224735-0.975915-0.748518$

$4.420131-0.284506-0.380874$

$5.581230-1.000044-0.085634$

$6.474886-0.452009 \quad 0.197383$

$5.625432-2.385670-0.115925$

$6.533681-2.921277 \quad 0.143346$

$4.479117-3.066425-0.494976$

$4.502292-4.150807-0.549113$

$3.302698-2.398703-0.834986$

$\begin{array}{lll}4.503114 & 1.230050-0.254088\end{array}$

$3.517038 \quad 1.642124-0.454384$

$5.475759 \quad 1.829677-1.283999$

$6.504618 \quad 1.512289-1.086244$

$5.453595 \quad 2.923341-1.251244$

$5.224809 \quad 1.516921-2.298015$

$\begin{array}{llll}4.919953 & 1.650055 & 1.164027\end{array}$

$\begin{array}{llll}4.215443 & 1.279762 & 1.909154\end{array}$

$\begin{array}{llll}4.966050 & 2.738673 & 1.248764\end{array}$

$\begin{array}{llll}5.909532 & 1.262302 & 1.421063\end{array}$

$2.160189-3.250490-1.362290$

$1.341390-2.576300-1.623666$

$2.576904-3.974263-2.653558$

$2.864578-3.257411-3.427439$

$1.760019-4.589258-3.041500$

$3.432272-4.633414-2.480931$

$1.653080-4.248679-0.315726$

$2.450994-4.922968 \quad 0.008931$

$0.848666-4.865857-0.725219$

$\begin{array}{lll}1.274298 & -3.729201 \quad 0.564303\end{array}$

$\begin{array}{llll}-0.409655 & 3.126169 & -0.217919\end{array}$

$\begin{array}{lll}0.461162 & 4.053720 & 0.416278\end{array}$

$\begin{array}{llll}-0.016235 & 5.301441 & 0.823334\end{array}$

$\begin{array}{lll}0.666636 & 6.001402 & 1.294861\end{array}$

$\begin{array}{llll}-1.346496 & 5.653340 & 0.666732\end{array}$

$\begin{array}{llll}-1.708517 & 6.620978 & 1.001486\end{array}$

$\begin{array}{llll}-2.215305 & 4.740281 & 0.081765\end{array}$

$-3.254880 \quad 5.019188-0.032196$

$\begin{array}{lll}-1.782574 & 3.493928 & -0.376356\end{array}$

$\begin{array}{lll}1.914580 & 3.719522 & 0.702901\end{array}$

$\begin{array}{llll}2.040982 & 2.655769 & 0.513349\end{array}$ 


\begin{tabular}{|c|c|c|c|}
\hline & 2.858490 & 4.4 & $0 ?$ \\
\hline & 2.667470 & 4.258024 & -1.273380 \\
\hline & 3.904214 & 4.261232 & -0.013735 \\
\hline & 2.727484 & 5.575776 & -0.099946 \\
\hline & 2.278262 & 3.982771 & 2.173322 \\
\hline & 2.256372 & 5.050158 & 2.413055 \\
\hline & 3.284160 & 3.619977 & 2.391301 \\
\hline & 1.587800 & 3.476388 & 2.848200 \\
\hline & -2.782133 & 2.579529 & -1.080718 \\
\hline & -2.690260 & 1.576820 & -0.654096 \\
\hline & -2.484502 & 2.449763 & -2.580271 \\
\hline & -2.558021 & 3.424060 & -3.074269 \\
\hline & -3.195330 & 1.766538 & -3.052718 \\
\hline & -1.486714 & 2.060629 & -2.747356 \\
\hline & -4.243847 & 3.009058 & -0.912840 \\
\hline & -4.448682 & 3.948499 & -1.436009 \\
\hline & -4.525011 & 3.138551 & 0.133807 \\
\hline & -4.902396 & 2.254557 & -1.344970 \\
\hline & $-1.917569-$ & -3.997484 & 0.511517 \\
\hline & $-2.372531-$ & $-3.316150-0$ & 0.207661 \\
\hline & -2.673566 & -4.747231 & 0.769061 \\
\hline & -1.101756 & -4.520628 & 0.010076 \\
\hline & -0.504302 & -4.480349 & 3.12 \\
\hline & 0.376541 & -4.891103 & 2.620608 \\
\hline & $-1.205390-$ & -5.306898 & 3.285082 \\
\hline & -0.184593 & -4.127572 & 4.106907 \\
\hline & -2.877349 & -2.586736 & 3.018077 \\
\hline & -2.629016 & -2.113731 & 3.971942 \\
\hline & $-3.538846-$ & -3.435447 & 3.226055 \\
\hline & $-3.447646-$ & -1.857668 & 2.437828 \\
\hline & 1.464977 & 0.503059 & 2.375358 \\
\hline & 2.051702 & 0.459007 & 1.456190 \\
\hline & 2.115152 & 0.909937 & 3.155878 \\
\hline & 0.671079 & 1.235071 & 2.214822 \\
\hline & 2.401965 & -2.276935 & 3.049901 \\
\hline & 2.172067 & -3.297878 & 3.366510 \\
\hline & 3.101976 & -1.853508 & 3.779314 \\
\hline & 2.926687 & -2.337574 & 2.092141 \\
\hline & 0.109296 & -1.003222 & 4.629744 \\
\hline & -0.770649 & -0.353392 & 4.611790 \\
\hline & 0.838258 & -0.555643 & 5.314719 \\
\hline & -0.203943 & -1.955951 & 5.066419 \\
\hline & $-4.742020-1$ & $1.101987-0$ & .885845 \\
\hline & $-5.716580-$ & -0.486269 & 2.389779 \\
\hline
\end{tabular}

$\begin{array}{llll}\mathrm{H} & -5.451295 & 0.540162 & -2.655241 \\ \mathrm{H} & -5.497350 & -1.111238 & -3.260975 \\ \mathrm{H} & -6.798069 & -0.517181 & -2.217178 \\ \mathrm{C} & -5.235152 & -2.902389 & -0.579861 \\ \mathrm{H} & -4.913093 & -3.550302 & -1.399737 \\ \mathrm{H} & -4.787330 & -3.288152 & 0.338532 \\ \mathrm{H} & -6.322875 & -2.993328 & -0.484139 \\ \mathrm{C} & -5.277222 & -0.127992 & 0.637309 \\ \mathrm{H} & -4.802874 & -0.525162 & 1.538234 \\ \mathrm{H} & -5.019898 & 0.928400 & 0.572340 \\ \mathrm{H} & -6.360790 & -0.196238 & 0.782744 \\ \mathrm{C} & -2.930538 & -0.996685 & -1.175184 \\ \mathrm{C} & -2.238717 & -1.322817 & -2.193269 \\ \mathrm{C} & -0.848721 & -0.703928 & -4.180058 \\ \mathrm{H} & 0.231241 & -0.759188 & -4.350122 \\ \mathrm{H} & -1.333499 & -0.959145 & -5.134973 \\ \mathrm{H} & -1.097772 & 0.331979 & -3.952729 \\ \mathrm{C} & -1.269477 & -1.647831 & -3.074787 \\ \mathrm{C} & -0.918821 & -3.103696 & -3.304736 \\ \mathrm{H} & -1.498240 & -3.526108 & -4.140151 \\ \mathrm{H} & 0.135102 & -3.214551 & -3.568295 \\ \mathrm{H} & -1.116240 & -3.720467 & -2.426159 \\ \mathrm{Si} & -2.537085 & 1.659616 & 2.665051 \\ \mathrm{H} & -1.921503 & 0.926265 & 1.532928 \\ \mathrm{H} & -3.709457 & 2.404743 & 2.150755 \\ \mathrm{C} & -1.326959 & 2.835446 & 3.481925 \\ \mathrm{H} & -0.491350 & 2.300736 & 3.937434 \\ \mathrm{H} & -0.928099 & 3.541966 & 2.752270 \\ \mathrm{H} & -1.827498 & 3.408322 & 4.268311 \\ \mathrm{H} & -3.026662 & 0.645276 & 3.632927 \\ & & & \end{array}$

TS2

Imaginary frequency: $-73.27 \mathrm{~cm}^{-1}$

\begin{tabular}{lcrr} 
Atom & $\mathrm{X}$ & $\mathrm{Y}$ & $\mathrm{Z}$ \\
$\mathrm{La}$ & -0.118150 & -0.346231 & 0.096629 \\
$\mathrm{Si}$ & 0.035288 & -3.273920 & 2.054731 \\
$\mathrm{Si}$ & 1.925293 & -1.074484 & 2.924431 \\
$\mathrm{~N}$ & \multicolumn{4}{c}{1.854798} & -0.250748 & -1.253686 \\
$\mathrm{~N}$ & 0.278579 & 1.983820 & -0.255237 \\
$\mathrm{~N}$ & 0.670147 & -1.680236 & 1.912796 \\
$\mathrm{C}$ & 2.125645 & 0.933766 & -3.442951 \\
$\mathrm{H}$ & 2.883317 & 0.163376 & -3.587506 \\
$\mathrm{H}$ & 2.573441 & 1.887249 & -3.732879 \\
$\mathrm{H}$ & 1.317582 & 0.736223 & -4.161498
\end{tabular}




\begin{tabular}{|c|c|c|c|c|c|}
\hline C & $1.640133 \quad 0.930023-2.007101$ & $\mathrm{C}$ & 2.410299 & 3.810307 & 0.668322 \\
\hline $\mathrm{C}$ & $0.871097 \quad 1.973615-1.545683$ & $\mathrm{H}$ & 2.477747 & 2.741714 & 0.471722 \\
\hline C & $0.478392 \quad 3.069921-2.519099$ & $\mathrm{C}$ & 3.134273 & 4.544427 & -0.469262 \\
\hline & $-0.236604 \quad 2.711260-3.271295$ & $\mathrm{H}$ & 2.721974 & 4.271705 & -1.440817 \\
\hline 5 & $1.333096 \quad 3.467210-3.071459$ & $\mathrm{H}$ & 4.200076 & 4.299504 & -0.474736 \\
\hline $\mathrm{H}$ & $0.013883 \quad 3.907242-2.000915$ & $\mathrm{H}$ & 3.041886 & 5.629474 & -0.355352 \\
\hline & $3.046564-0.984031-1.374464$ & $\mathrm{C}$ & 3.097927 & 4.093441 & 2.013060 \\
\hline & $4.324658-0.414933-1.132492$ & $\mathrm{H}$ & 3.163578 & 5.166567 & 2.216137 \\
\hline 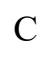 & $5.462453-1.224798-1.158042$ & $\mathrm{H}$ & 4.116530 & 3.698396 & 2.016566 \\
\hline $\mathrm{H}$ & $6.430584-0.777190-0.954067$ & $\mathrm{H}$ & 2.553923 & 3.629443 & 2.836744 \\
\hline & $5.385911-2.585665-1.407833$ & $\mathrm{C}$ & -2.570802 & 2.707037 & 0.022558 \\
\hline $\mathrm{H}$ & $6.278938-3.203082-1.396327$ & $\mathrm{H}$ & -2.181171 & 1.691603 & -0.066147 \\
\hline $\mathrm{C}$ & $4.143279-3.144609-1.682134$ & $\mathrm{C}$ & -3.113646 & 3.103299 & -1.358225 \\
\hline $\mathrm{H}$ & $4.082107-4.203242-1.908392$ & $\mathrm{H}$ & -3.566508 & 4.099173 & -1.320208 \\
\hline $\mathrm{C}$ & $2.982866-2.373148-1.692814$ & $\mathrm{H}$ & -3.876843 & 2.392856 & -1.688650 \\
\hline $\mathrm{C}$ & $4.511042 \quad 1.061266-0.819470$ & $\mathrm{H}$ & -2.322111 & 3.131555 & -2.108112 \\
\hline $\mathrm{H}$ & $3.529498 \quad 1.529484-0.818136$ & $\mathrm{C}$ & -3.710992 & 2.664749 & 1.044120 \\
\hline 0 & $5.349942 \quad 1.753755-1.905756$ & $\mathrm{H}$ & -4.281924 & 3.597935 & 1.055769 \\
\hline $\mathrm{H}$ & $6.367550 \quad 1.352003-1.940413$ & $\mathrm{H}$ & -3.335385 & 2.492918 & 2.052807 \\
\hline $\mathrm{H}$ & $5.423486 \quad 2.828375-1.716608$ & $\mathrm{H}$ & -4.409711 & 1.865129 & 0.799940 \\
\hline $\mathrm{H}$ & $4.906438 \quad 1.615359-2.893634$ & $\mathrm{C}$ & $-1.042572-$ & -3.657962 & 0.542892 \\
\hline 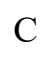 & $\begin{array}{lll}5.135652 & 1.274888 & 0.566367\end{array}$ & $\mathrm{H}$ & -1.928341 & -3.019914 & 0.495041 \\
\hline $\mathrm{H}$ & $\begin{array}{lll}4.500762 & 0.865012 & 1.352063\end{array}$ & $\mathrm{H}$ & $-1.412116-$ & -4.686604 & 0.607862 \\
\hline $\mathrm{H}$ & $\begin{array}{lll}5.275400 & 2.340625 & 0.764605\end{array}$ & $\mathrm{H}$ & -0.507391 & $-3.588257-$ & 0.405858 \\
\hline $\mathrm{H}$ & $\begin{array}{lll}6.114760 & 0.793840 & 0.646722\end{array}$ & $\mathrm{C}$ & 1.402669 & -4.587731 & 2.133367 \\
\hline $\mathrm{C}$ & $1.685113-3.005074-2.175738$ & $\mathrm{H}$ & 2.152436 & -4.430190 & 1.353817 \\
\hline $\mathrm{H}$ & $0.862507-2.495050-1.669640$ & $\mathrm{H}$ & 0.997234 & -5.598841 & 2.016243 \\
\hline $\mathrm{C}$ & $1.516905-2.747745-3.682470$ & $\mathrm{H}$ & 1.926952 & -4.558398 & 3.094020 \\
\hline $\mathrm{H}$ & $1.475974-1.680184-3.896309$ & $\mathrm{C}$ & $-1.074149-$ & -3.550336 & 3.558602 \\
\hline $\mathrm{H}$ & $0.602238-3.208708-4.068102$ & $\mathrm{H}$ & -0.554297 & -3.337604 & 4.496256 \\
\hline $\mathrm{H}$ & $2.364463-3.168293-4.231901$ & $\mathrm{H}$ & -1.422329 & -4.588942 & 3.600497 \\
\hline $\mathrm{C}$ & $1.550340-4.500770-1.877919$ & $\mathrm{H}$ & -1.957075 & -2.909450 & 3.515795 \\
\hline $\mathrm{H}$ & $2.245569-5.097646-2.475229$ & $\mathrm{C}$ & 1.927186 & 0.811279 & 2.807074 \\
\hline 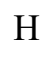 & $0.542532-4.845379-2.125525$ & $\mathrm{H}$ & 2.026296 & 1.178773 & 1.782424 \\
\hline $\mathrm{H}$ & $1.731701-4.723586-0.825814$ & $\mathrm{H}$ & 2.760557 & 1.237199 & 3.373630 \\
\hline $\mathrm{C}$ & $\begin{array}{lll}-0.064777 & 3.238059 & 0.301576\end{array}$ & $\mathrm{H}$ & 1.006420 & 1.240077 & 3.216569 \\
\hline & $\begin{array}{lll}0.933430 & 4.159256 & 0.715225\end{array}$ & $\mathrm{C}$ & 3.631047 & -1.715009 & 2.421275 \\
\hline 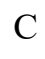 & $\begin{array}{lll}0.564752 & 5.418467 & 1.191677\end{array}$ & $\mathrm{H}$ & 3.716422 & -2.788884 & 2.610714 \\
\hline $\mathrm{H}$ & $\begin{array}{lll}1.338463 & 6.119575 & 1.489224\end{array}$ & $\mathrm{H}$ & 4.437131 & -1.219162 & 2.973638 \\
\hline & $-0.766808 \quad 5.790602 \quad 1.304058$ & $\mathrm{H}$ & 3.814211 & -1.563409 & 1.355102 \\
\hline & $\begin{array}{lll}-1.036856 & 6.773952 & 1.677784\end{array}$ & $\mathrm{C}$ & 1.691202 & -1.494749 & 4.758595 \\
\hline & $\begin{array}{lll}-1.748909 & 4.878374 & 0.945604\end{array}$ & $\mathrm{H}$ & 0.715287 & -1.157262 & 5.119571 \\
\hline & $\begin{array}{lll}-2.792433 & 5.159765 & 1.040128\end{array}$ & $\mathrm{H}$ & 2.459947 & -1.009649 & 5.370636 \\
\hline & $\begin{array}{lll}-1.427910 & 3.612849 & 0.449842\end{array}$ & $\mathrm{H}$ & 1.757772 & -2.570523 & 4.947966 \\
\hline
\end{tabular}




$\begin{array}{llll}\mathrm{Si} & -5.963168 & -0.557508 & -0.935780 \\ \mathrm{C} & -6.025559 & -0.619966 & -2.826686 \\ \mathrm{H} & -5.483526 & 0.222013 & -3.267742 \\ \mathrm{H} & -5.564409 & -1.536130 & -3.207035 \\ \mathrm{H} & -7.055507 & -0.584271 & -3.197861 \\ \mathrm{C} & -6.902927 & -2.041598 & -0.246673 \\ \mathrm{H} & -6.455317 & -2.979387 & -0.588817 \\ \mathrm{H} & -6.870779 & -2.040977 & 0.845065 \\ \mathrm{H} & -7.952874 & -2.035172 & -0.559076 \\ \mathrm{C} & -6.817614 & 1.030060 & -0.375756 \\ \mathrm{H} & -6.790912 & 1.126477 & 0.711566 \\ \mathrm{H} & -6.343362 & 1.917933 & -0.802762 \\ \mathrm{H} & -7.867401 & 1.031508 & -0.689361 \\ \mathrm{C} & -4.192214 & -0.641314 & -0.340325 \\ \mathrm{C} & -3.285909 & -0.817051 & -1.242306 \\ \mathrm{C} & -1.697215 & -0.009649 & -2.994491 \\ \mathrm{H} & -0.617644 & -0.163572 & -3.094844 \\ \mathrm{H} & -2.105100 & -0.017023 & -4.014039 \\ \mathrm{H} & -1.856314 & 0.985432 & -2.583373 \\ \mathrm{C} & -2.357615 & -1.085650 & -2.155753 \\ \mathrm{C} & -2.173638 & -2.509506 & -2.645071 \\ \mathrm{H} & -2.601732 & -2.622954 & -3.650601 \\ \mathrm{H} & -1.118785 & -2.782746 & -2.725072 \\ \mathrm{H} & -2.660112 & -3.226273 & -1.984603 \\ \mathrm{Si} & -3.765916 & -0.821704 & 1.668183 \\ \mathrm{H} & -2.265513 & -0.603691 & 1.154360 \\ \mathrm{H} & -5.219935 & -0.399434 & 1.946635 \\ \mathrm{C} & -3.160334 & -0.211165 & 3.405405 \\ \mathrm{H} & -2.156738 & -0.584376 & 3.635763 \\ \mathrm{H} & -3.115328 & 0.882658 & 3.448262 \\ \mathrm{H} & -3.853940 & -0.531364 & 4.192879 \\ \mathrm{H} & -3.810561 & -2.313734 & 1.762684 \\ & & & \end{array}$

\begin{tabular}{|c|c|c|c|}
\hline & 1.180166 & 0.85 & 74 \\
\hline 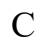 & 1.521606 & 1.014245 & .986971 \\
\hline & .711788 & 2.018961 & -1.509484 \\
\hline & 0.291523 & 3.116812 & -2.471198 \\
\hline & -0.374766 & 2.740800 & 3.258838 \\
\hline 11 & 1.142373 & 3.5734 & -2.982809 \\
\hline & -0.234326 & 3.915255 & 50834 \\
\hline & 3.024224 & 0.83993 & 4129 \\
\hline C & 4.279775 & 0.20783 & 7695 \\
\hline E & 5.4558 & 0.95 & 502 \\
\hline & 6.405342 & -0.462 & 944 \\
\hline $\mathrm{C}$ & 5.441794 & 2.3226 & 2798 \\
\hline $\mathrm{H}$ & 6.364698 & -2.89444 & 15879 \\
\hline 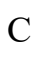 & 4.221123 & 2.94371 & 9163 \\
\hline & 4.207 & -4.004 & 716 \\
\hline & 3.023620 & 2.231 & 318 \\
\hline$C$ & 4.405797 & 1.273294 & -0.877181 \\
\hline & 3.404627 & 1.696841 & 0.848240 \\
\hline & 5.187775 & 2.013 & 4206 \\
\hline $\mathrm{H}$ & 6.222728 & 1.662366 & -2.032417 \\
\hline 11 & 5.213338 & 3.089459 & -1.777280 \\
\hline 11 & 4.732024 & 1.861846 & -2.954319 \\
\hline & 5.054149 & 1.501117 & 0.495766 \\
\hline $\mathrm{H}$ & 4.464434 & 1.044319 & $1.29067^{\prime}$ \\
\hline $\mathbf{H}$ & 5.140980 & 2.569819 & 0.70805 \\
\hline & 6.059853 & 1.073479 & 0.54231 \\
\hline & 1.748804 & 2.92992 & 171710 \\
\hline $\mathrm{H}$ & 0.912421 & -2.480944 & 630353 \\
\hline $\mathrm{C}$ & 1.522995 & 2.65796 & 68437 \\
\hline 11 & 1.417022 & -1.59094 & 61873 \\
\hline U & 0.626058 & -3.16403 & 038797 \\
\hline $\mathrm{H}$ & 2.377614 & -3.020472 & 4.247378 \\
\hline$C$ & 1.713433 & 4.435875 & 898324 \\
\hline & 2.427501 & $-4.978303-$ & 24575 \\
\hline $\mathrm{H}$ & 0.722486 & $-4.837988-$ & 2.124890 \\
\hline $\mathrm{H}$ & 1.935806 & $-4.663357-$ & 0.854822 \\
\hline & -0.259289 & 3.237960 & 0.346592 \\
\hline $\mathrm{C}$ & 0.715655 & 4.184269 & 0.76005 \\
\hline 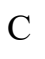 & 0.317332 & 5.430802 & 1.24636 \\
\hline $\mathrm{H}$ & 1.075095 & 6.149953 & 1.54238 \\
\hline 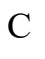 & -1.022162 & 5.767125 & 1.371764 \\
\hline $\mathrm{H}$ & -1.315488 & 6.740593 & 1.753819 \\
\hline $\mathrm{C}$ & -1.981991 & 4.830364 & 1.015493 \\
\hline $\mathrm{H}$ & -3.031817 & 5.081506 & 1.122129 \\
\hline
\end{tabular}




\begin{tabular}{|c|c|c|c|}
\hline & 1.631127 & 3.577781 & 0 \\
\hline & 2.201521 & 3.877770 & 0.706949 \\
\hline & 2.300071 & 2.818481 & 0.478228 \\
\hline & 2.908542 & 4.667466 & -0.40 \\
\hline & 2.506153 & 4.415377 & -1.384944 \\
\hline & 3.980524 & 4.451947 & -0.413494 \\
\hline & 2.786857 & 5.74 & -0.255938 \\
\hline & 2.874303 & 4.138621 & 2.063863 \\
\hline & 2.906159 & 5.206491 & 2.300252 \\
\hline & 3.903946 & 3.773321 & 2.062056 \\
\hline & 2.339259 & 3.633851 & 2.869219 \\
\hline & -2.751654 & 2.644367 & 0.082001 \\
\hline & -2.350794 & 1.629899 & 0.058640 \\
\hline & -3.235405 & 2.960562 & -1.340818 \\
\hline & -3.683391 & 3.958663 & 378120 \\
\hline & -3.988356 & 2.234359 & 0542 \\
\hline & -2.414691 & 2.942067 & -2.057421 \\
\hline & -3.941831 & 2.650445 & 1.048833 \\
\hline & -4.560232 & 3.54 & 0.91 \\
\hline & -3.619781 & 2.614384 & 2.089260 \\
\hline & -4.579976 & 1.787406 & 0.869944 \\
\hline & $-0.824198-$ & -3.973726 & 0.580426 \\
\hline & -1.8218 & 1 & 12 \\
\hline & -0.934554 & -5.063178 & 0.616083 \\
\hline & -0.378086 & $-3.741752-$ & 0.388259 \\
\hline & $1.6508^{\prime}$ & -4.61 & 854 \\
\hline & 2.368186 & -4.513281 & 1.416177 \\
\hline & 1.290448 & -5.653987 & 2.235546 \\
\hline & 2.203186 & -4.467553 & 3.167421 \\
\hline & $-0.895987-$ & -3.614074 & 3.571306 \\
\hline & -0.387182 & -3.339093 & 4.498797 \\
\hline & -1.227032 & -4.654495 & 3.669185 \\
\hline & -1.792145 & -2.994731 & 3.483697 \\
\hline & 1.929156 & 0.778601 & 2.702801 \\
\hline & 2.009100 & 1.151661 & 1.678455 \\
\hline & 2.742132 & 1.247134 & 3.264905 \\
\hline & 0.991242 & 1.163884 & 3.117506 \\
\hline & 3.757051 & -1.652576 & 2.298314 \\
\hline & 3.903857 & -2.720600 & 2.482097 \\
\hline & 4.541847 & -1.114601 & 2.842083 \\
\hline & 3.916594 & -1.486341 & 1.230871 \\
\hline & 1.860086 & -1.507567 & 4.675697 \\
\hline & 0.887207 & -1.189884 & 5.062234 \\
\hline & 2.633393 & -0.991437 & 5.255899 \\
\hline
\end{tabular}

\begin{tabular}{|c|c|}
\hline & 74 \\
\hline & $-5.909197-0.735822-0.781853$ \\
\hline & $-6.056276-0.857694-2.6$ \\
\hline & $\begin{array}{ll}-5.588264 & 0.001262-3.153966\end{array}$ \\
\hline & $-5.561370-1.756030-3.043640$ \\
\hline & $-7.103811-0.892290-2.9$ \\
\hline & $-6.707699-2.254473 \quad 0.0$ \\
\hline & $-6.228003-3.173401-0.347943$ \\
\hline & $-6.603724-2.221668 \quad 1.088211$ \\
\hline & $-7.774500-2.318165-0.238588$ \\
\hline & $-6.843612 \quad 0.803395-0.221188$ \\
\hline & $\begin{array}{lll}-6.786912 & 0.924157 & 0.8\end{array}$ \\
\hline & $-6.451951 \quad 1.713608-0.682305$ \\
\hline & $-7.900671 \quad 0.719652-0.497629$ \\
\hline & $-4.094128-0.73$ \\
\hline & -3.2 \\
\hline & $-1.705119-0.090818-3.066116$ \\
\hline & $-0.624050-0.238786-3.144419$ \\
\hline & $-2.099754-0.12552$ \\
\hline & $\begin{array}{lll}-1.879203 & 0.909532 & -2.67\end{array}$ \\
\hline & $-2.363310-1.155857-2.216194$ \\
\hline 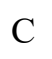 & $-2.164540-2.591478-2.661485$ \\
\hline & $-2.583220-2.742191-3.665269$ \\
\hline & $-1.104998-2.850787-2.72$ \\
\hline & $-2.644377-3.291342-1.978266$ \\
\hline S & $\begin{array}{ll}-3.541493-0.949170 & 1.585215\end{array}$ \\
\hline & $-2.091091-1.390523 \quad 0.913794$ \\
\hline & $-4.926446-0.438920 \quad 2.077515$ \\
\hline & $-2.495413 \quad 0.141700 \quad 2.784905$ \\
\hline$\theta$ & $-1.718405-0.4835793 .236566$ \\
\hline & $\begin{array}{lll}-1.983707 & 0.973120 & 2.283736\end{array}$ \\
\hline & $\begin{array}{lll}-3.099114 & 0.589812 & 3.579232\end{array}$ \\
\hline & $-3.716428-2.369186$ \\
\hline
\end{tabular}

8

$\begin{array}{lccc}\text { Atom } & \mathrm{X} & \mathrm{Y} & \mathrm{Z} \\ \mathrm{Si} & 1.743830 & -0.525037 & 0.018004 \\ \mathrm{C} & 1.461130 & -2.370169 & -0.252784 \\ \mathrm{H} & 0.770301 & -2.774623 & 0.495297 \\ \mathrm{H} & 1.026094 & -2.560967 & -1.240388 \\ \mathrm{H} & 2.401868 & -2.929221 & -0.183713 \\ \mathrm{C} & 2.861399 & 0.180302 & -1.332523 \\ \mathrm{H} & 2.420549 & 0.036333 & -2.325660 \\ \mathrm{H} & 3.023368 & 1.256038 & -1.195401\end{array}$




\begin{tabular}{|c|c|}
\hline $\mathrm{H}$ & $3.843886-0.307177-1.32$ \\
\hline $\mathrm{C}$ & $2.530812-0.221913 \quad 1.709099$ \\
\hline & $\begin{array}{lll}2.654687 & 0.850365 & 1.899420\end{array}$ \\
\hline & $1.906555-0.632349 \quad 2.511245$ \\
\hline & $\begin{array}{lll}3.519561 & -0.691821 & 1.777783\end{array}$ \\
\hline & $0.083915 \quad 0.370261-0.044697$ \\
\hline & $-1.024456-0.329407-0.030943$ \\
\hline & $-2.791175-1.499279-1.296911$ \\
\hline & $-2.863284-2.595479-1.319324$ \\
\hline & $-3.815634-1.109374-1.374053$ \\
\hline & $-2.232984-1.174827-2.179312$ \\
\hline & $-2.134107-1.032040-0.016506$ \\
\hline
\end{tabular}

$\begin{array}{lccc}\mathrm{C} & -2.811452 & -1.413324 & 1.281762 \\ \mathrm{H} & -3.835643 & -1.016847 & 1.317462 \\ \mathrm{H} & -2.887619 & -2.505517 & 1.375271 \\ \mathrm{H} & -2.265856 & -1.032318 & 2.149364 \\ \mathrm{Si} & -0.054303 & 2.240351 & -0.110441 \\ \mathrm{H} & 0.819141 & 2.833384 & 0.944874 \\ \mathrm{C} & -1.838915 & 2.796507 & 0.126421 \\ \mathrm{H} & -2.484697 & 2.349223 & -0.636839 \\ \mathrm{H} & -2.215655 & 2.481338 & 1.105527 \\ \mathrm{H} & -1.929329 & 3.886139 & 0.058219 \\ \mathrm{H} & 0.468413 & 2.737692 & -1.416691\end{array}$

$\begin{array}{lll}\text { C } & -2.811452-1.413324 & 1.281762\end{array}$

H $\quad-3.835643-1.016847 \quad 1.317462$

$\mathrm{H} \quad-2.887619-2.505517 \quad 1.375271$

H $\quad-2.265856-1.032318 \quad 2.149364$

Si $\quad-0.054303 \quad 2.240351-0.110441$

$\mathrm{H} \quad 0.819141 \quad 2.833384 \quad 0.944874$

$\begin{array}{llll}C & -1.838915 & 2.796507 & 0.126421\end{array}$

$\begin{array}{llll}-2.484697 & 2.349223 & -0.636839\end{array}$

$\begin{array}{lll}-1.929329 & 3.886139 & 0.058219\end{array}$

$\begin{array}{lll}0.468413 & 2.737692-1.416691\end{array}$ 


\section{Kinetic Studies}

In the first place, the experiments were operated with the same initial concentration of the hydrosilane and enyne $(0.5 \mathrm{M})$ (Table S2). The results indicated that the total order of the hydrosilane and enyne was the second order (Figure S3, above plot), based on the same decreasing rate of [hydrosilane] and [enyne] concentrations in the hydrosilylation reaction. Then, the experiments were carried out to monitor the decrease of the enyne concentrations, in which the hydrosilane concentration (2.78 M, under approximately pseudo-zero-order condition in hydrosilane) was maintained in 10-fold excess over [enyne $]_{0}(0.278 \mathrm{M})$. It was observed that the enyne decay followed the first order behavior (Figure S3, below plot).

Table S2. Measurement of the 1,3-enyne 7g concentration at different times during the hydrosilylation process.

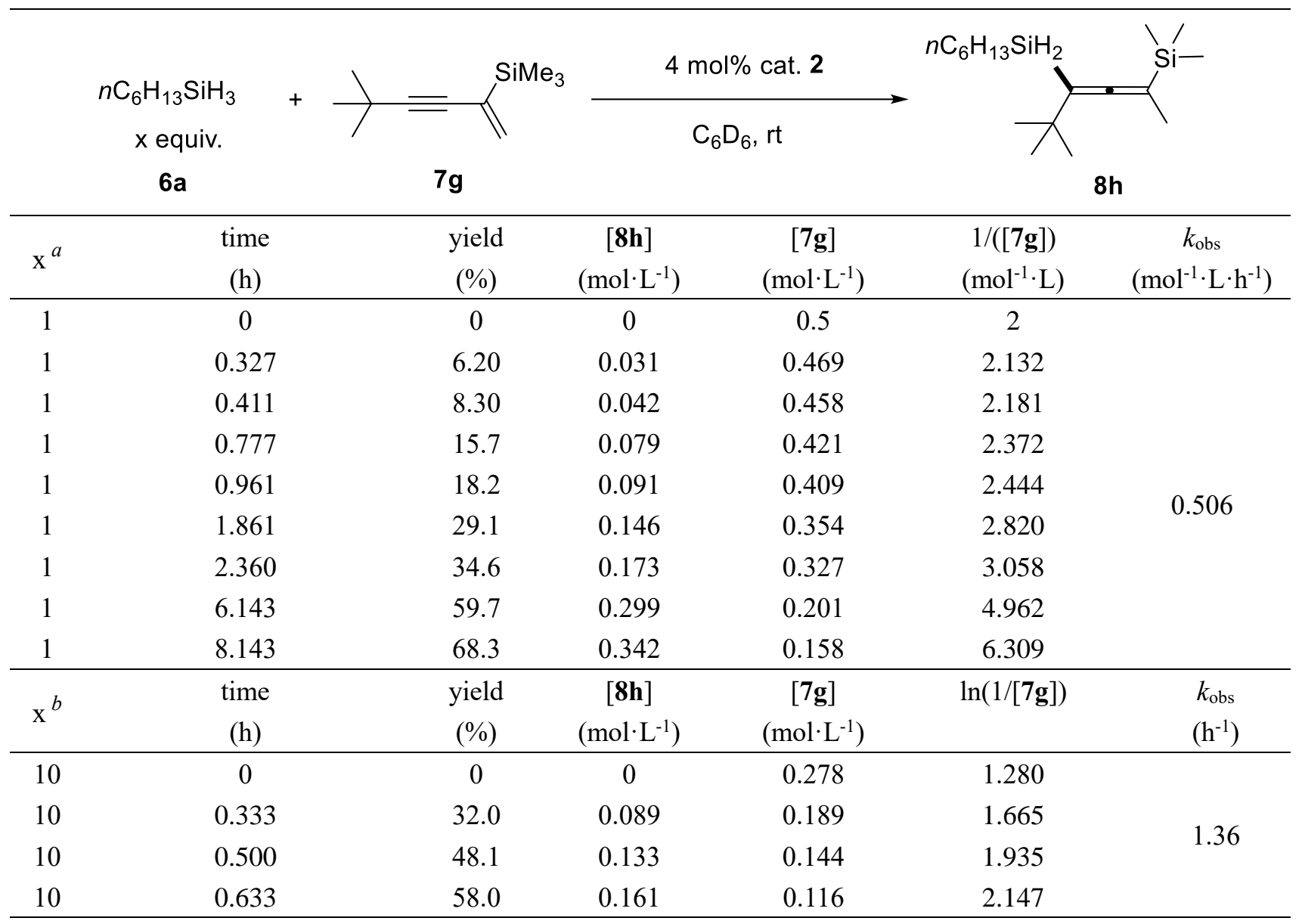


${ }^{a}$ Reaction conditions: $n \mathrm{C}_{6} \mathrm{H}_{13} \mathrm{SiH}_{3}$ 6a $(0.20 \mathrm{mmol}, 0.5 \mathrm{M})$, enyne $7 \mathrm{~g}(0.20 \mathrm{mmol}, 0.5 \mathrm{M})$, catalyst 2 (0.008 mmol, $4 \mathrm{~mol} \%$ ), room temperature of $23{ }^{\circ} \mathrm{C}$ and $0.4 \mathrm{~mL} \mathrm{C}_{6} \mathrm{D}_{6}$. The yield was recorded by ${ }^{1} \mathrm{H}$ NMR spectroscopy.

${ }^{b}$ Reaction conditions: $n \mathrm{C}_{6} \mathrm{H}_{13} \mathrm{SiH}_{3}$ 6a $(2.0 \mathrm{mmol}, 0.32 \mathrm{~mL}, 2.78 \mathrm{M})$, enyne $7 \mathbf{g}(0.20 \mathrm{mmol}, 0.278$ $\mathrm{M})$, catalyst $2(0.008 \mathrm{mmol}, 4 \mathrm{~mol} \%)$, room temperature of $23{ }^{\circ} \mathrm{C}$ and $0.4 \mathrm{~mL} \mathrm{C}_{6} \mathrm{D}_{6}$ (the total volume of reaction solution is $0.72 \mathrm{~mL}$ ). The yield was recorded by ${ }^{1} \mathrm{H}$ NMR spectroscopy.
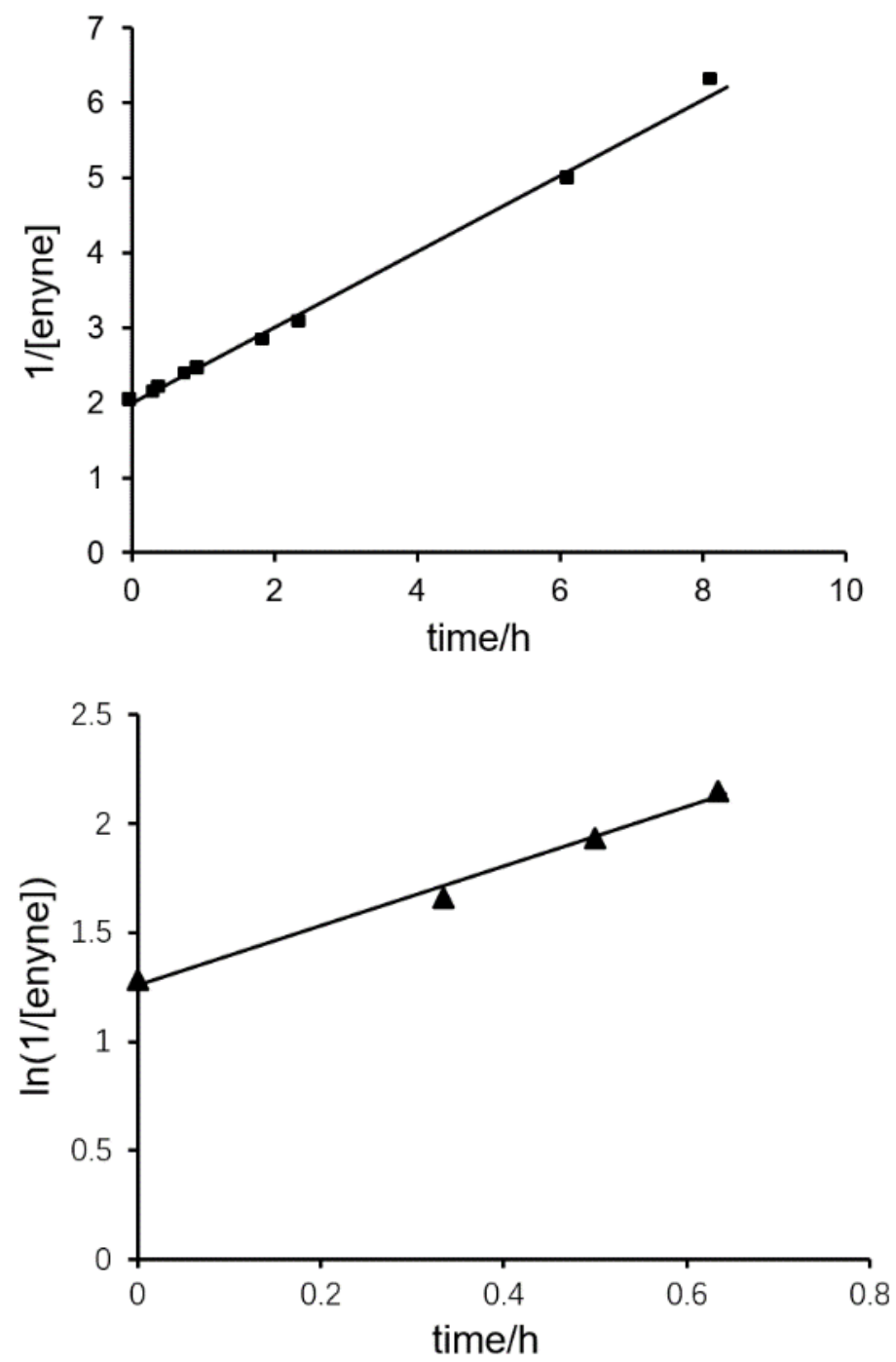

Figure S3. Kinetic plots for the catalytic hydrosilylation of enyne $7 \mathbf{g}$ with $n \mathrm{C}_{6} \mathrm{H}_{13} \mathrm{SiH}_{3} \mathbf{6 a}$ enabled by 2: (above) Initial concentration $[\text { hydrosilane }]_{0}=[\text { enyne }]_{0}=0.5 \mathrm{M}$; (below) Initial concentration $[\text { hydrosilane }]_{0}=2.78 \mathrm{M}$, [enyne $]_{0}=0.278 \mathrm{M}$. 


\section{References}

[S1] Mahrova, T. V.; Fukin, G. K.; Cherkasov, A. V.; Trifonov, A. A.; Ajellal, N.; Carpentier, J.-F. Inorg. Chem. 2009, 48, 4258.

[S2] Kissel, A. A.; Mahrova, T. V.; Lyubov, D. M.; Cherkasov, A. V.; Fukin, G. K.; Trifonov, A. A.; Rosal, I. D.; Maron, L. Dalton Trans. 2015, 44, 12137.

[S3] Liu, J.; Chen, W.; Li, J.; Cui, C. ACS Catal. 2018, 8, 2230.

[S4] Chen, W.; Song, H.; Li, J.; Cui, C. Angew. Chem. Int. Ed. 2020, 59, 2365.

[S5] Visco, M. D.; Wieting, J. M.; Mattson, A. E. Org. Lett. 2016, 18, 2883.

[S6] Ockey, D. A.; Lewis, M. A.; Schore, N. E. Tetrahedron 2003, 59, 5377.

[S7] Mizoguchi, H.; Micalizio, G. C. J. Am. Chem. Soc. 2015, 137, 6624.

[S8] Adamson, N. J.; Jeddi, H.; Malcolmson, S. J. J. Am. Chem. Soc. 2019, 141, 8574.

[S9] (a) Becke, A. D. J. Chem. Phys. 1993, 98, 5648. (b) Raghavachari, K. Theor. Chem. Acc. 2000, 103, 361. (c) Becke, A. D. J. Chem. Phys. 1993, 98, 1372. (d) Lee, C.; Yang, W.; Parr, R. G. Phys. Rev. $B$ 1988, 37, 785 .

[S10] Grimme, S.; Ehrlich, S.; Goerigk, L. J. Comput. Chem. 2011, 32, 1456.

[S11] (a) Dolg, M.; Stoll, H.; Savin, A.; Preuss, H. Theor. Chim. Acta. 1989, 75, 173. (b) Yang, J.; Dolg, M.; Theor. Chem. Acc. 2005, 113, 212. (c) Weigand, A.; Cao, X.; Yang, J.; Dolg, M. Theor. Chem. Acc. 2009, 126, 117. (d) Dolg, M.; Stoll, H.; Preuss, H. Theor. Chim. Acta. 1993, 85, 441.

[S12] Fukui, K. Acc. Chem. Res. 1981, 14, 363.

[S13] (a) Adamo, C.; Barone, V. J. Chem. Phys. 1999, 110, 6158. (b) Ernzerhof, M.; Scuseria, G. E; J. Chem. Phys. 1999, 110, 5029.

[S14] (a) Papajak, E.; Zheng, J.; Xu, X.; Leverentz, H. R.; Truhlar, D. G. J. Chem. Theory Comput. 2011,7, 3027. (b) Zheng, J.; Xu, X.; Truhlar, D. G. Theor. Chem. Acc. 2011, 128, 295.

[S15] Weigend, F.; Ahlrichs, R. Phys. Chem. Chem. Phys. 2005, 7, 3297.

[S16] Marenich, A. V.; Cramer, C. J.; Truhlar, D. G. J. Phys. Chem. B 2009, 113, 6378.

[S17] Frisch, M. J.; Trucks, G. W.; Schlegel, H. B.; Scuseria, G. E.; Robb, M. A.; Cheeseman, J. R.; Scalmani, G.; Barone, V.; Petersson, G. A.; Nakatsuji, H.; Li, X.; Caricato, M.; Marenich, A. V.; Bloino, J.; Janesko, B. G.; Gomperts, R.; Mennucci, B.; Hratchian, H. P.; Ortiz, J. V.; Izmaylov, A. F.; Sonnenberg, J. L.; Williams-Young, D.; Ding, F.; Lipparini, F.; Egidi, F.; Goings, J.; Peng, 
B.; Petrone, A.; Henderson, T.; Ranasinghe, D.; Zakrzewski, V. G.; Gao, J.; Rega, N.; Zheng, G.; Liang, W.; Hada, M.; Ehara, M.; Toyota, K.; Fukuda, R.; Hasegawa, J.; Ishida, M.; Nakajima, T.; Honda, Y.; Kitao, O.; Nakai, H.; Vreven, T.; Throssell, K.; Montgomery, J. A.; Jr., Peralta, J. E.; Ogliaro, F.; Bearpark, M. J.; Heyd, J. J.; Brothers, E. N.; Kudin, K. N.; Staroverov, V. N.; Keith, T. A.; Kobayashi, R.; Normand, J.; Raghavachari, K.; Rendell, A. P.; Burant, J. C.; Iyengar, S. S.; Tomasi, J.; Cossi, M.; Millam, J. M.; Klene, M.; Adamo, C.; Cammi, R.; Ochterski, J. W.; Martin, R. L.; Morokuma, K.; Farkas, O.; Foresman, J. B.; Fox, D. J. Gaussian 16, Revision A.03; Gaussian, Inc.: Wallingford, CT, 2016.

[S18] Legault, C. Y. CYLview, 1.0b; Université de Sherbrooke, 2009 (http://www.cylview.org). 


\section{NMR Spectra}

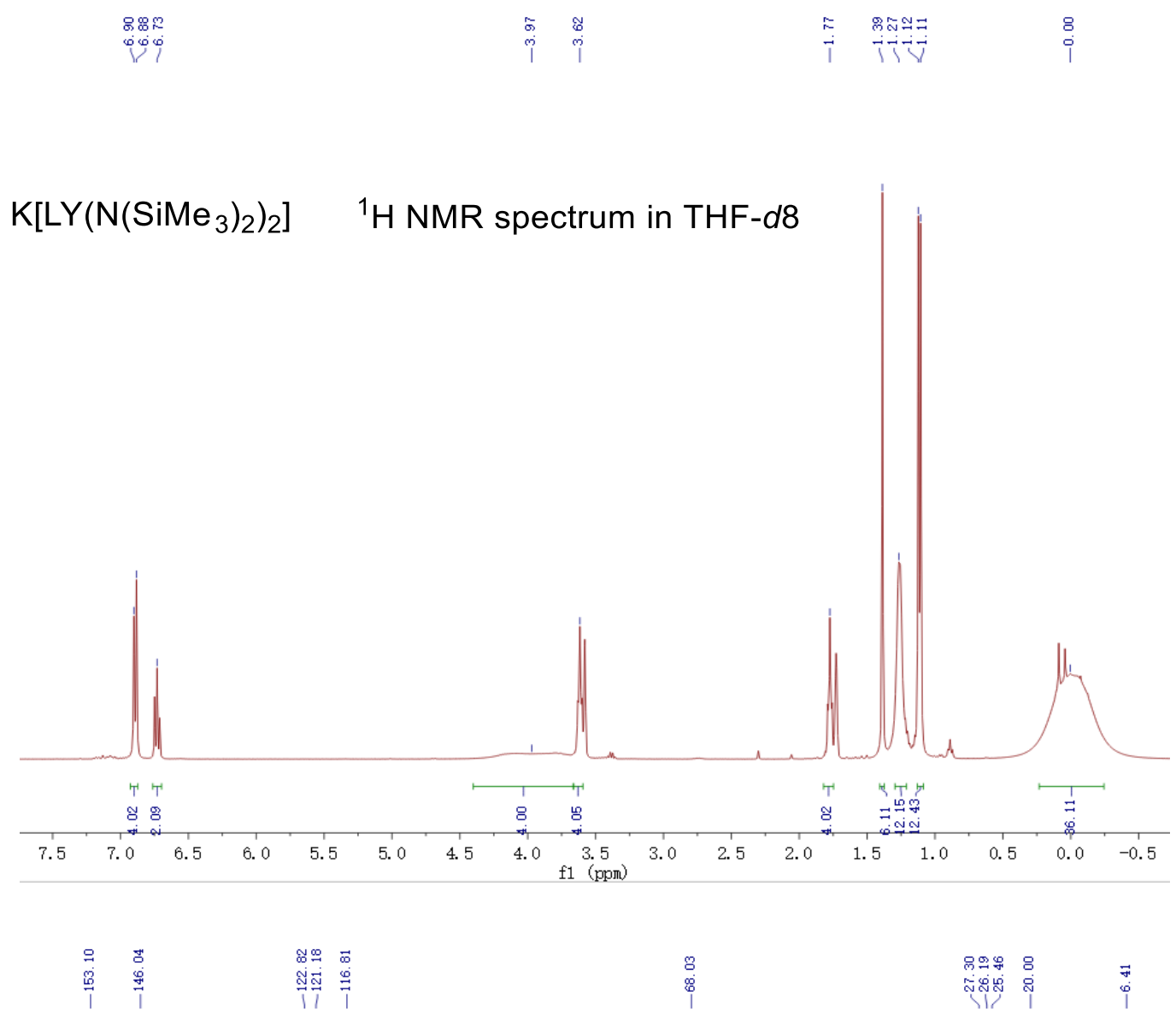

$\mathrm{K}\left[\mathrm{LY}\left(\mathrm{N}\left(\mathrm{SiMe}_{3}\right)_{2}\right)_{2}\right] \quad{ }^{13} \mathrm{C}$ NMR spectrum in THF-d8

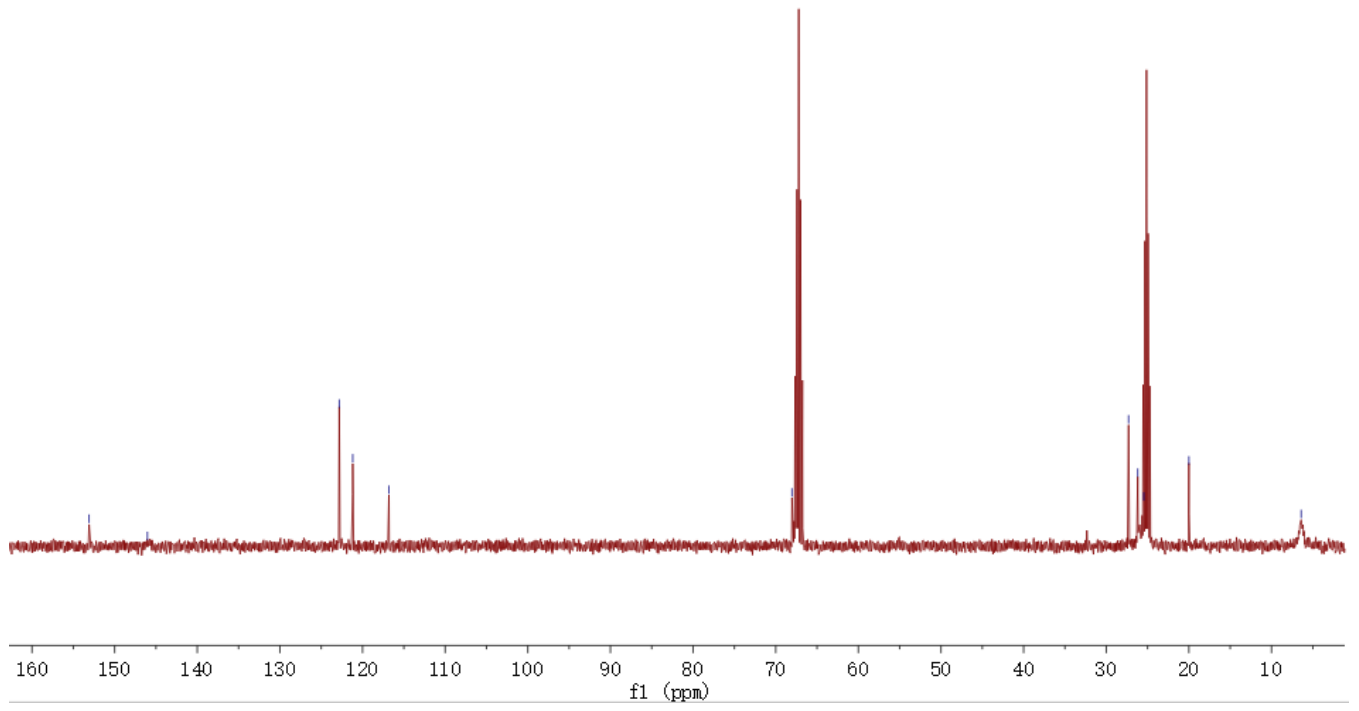




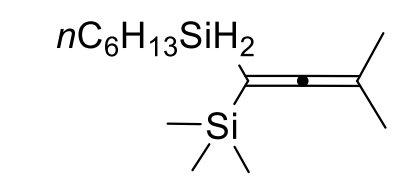

$8 a$

${ }^{1} \mathrm{H}$ NMR spectrum in $\mathrm{CDCl}_{3}$

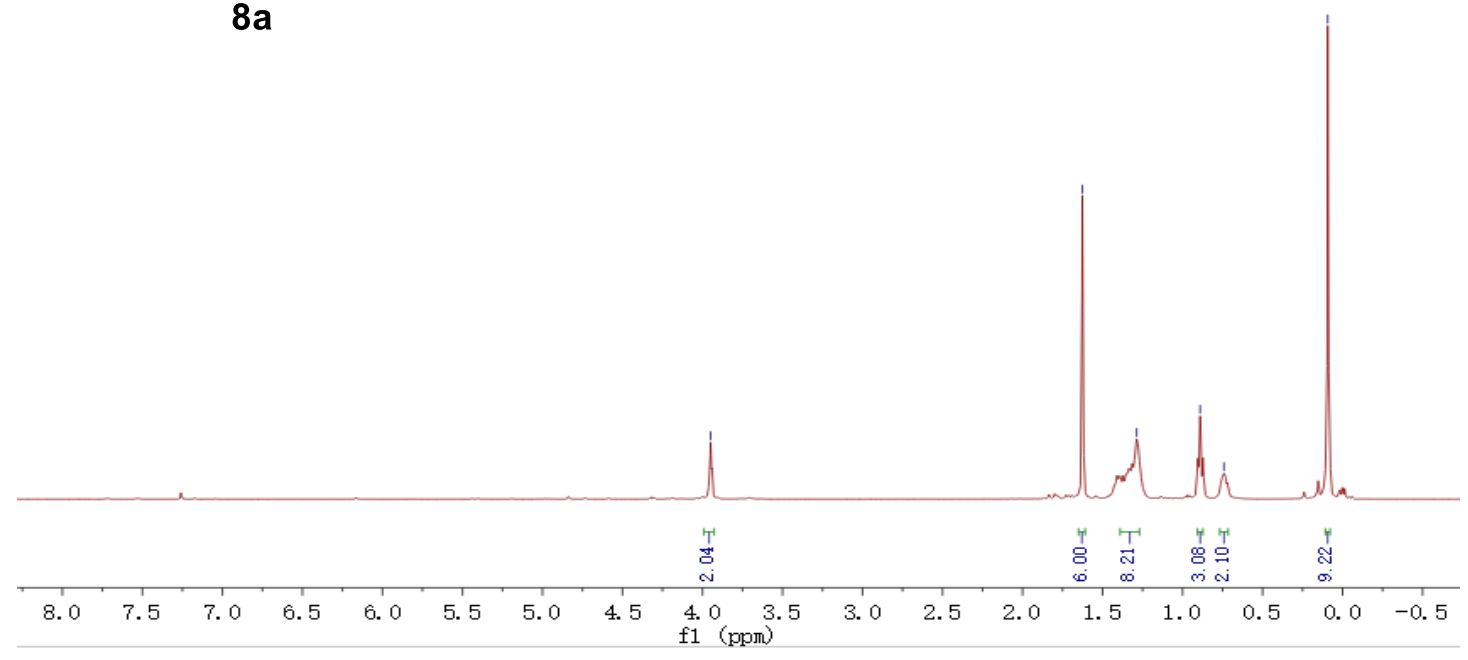

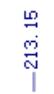

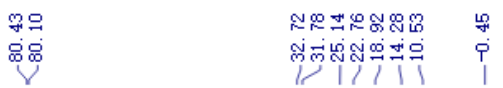

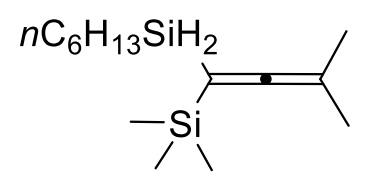

${ }^{13} \mathrm{C}$ NMR spectrum in $\mathrm{CDCl}_{3}$

$8 a$

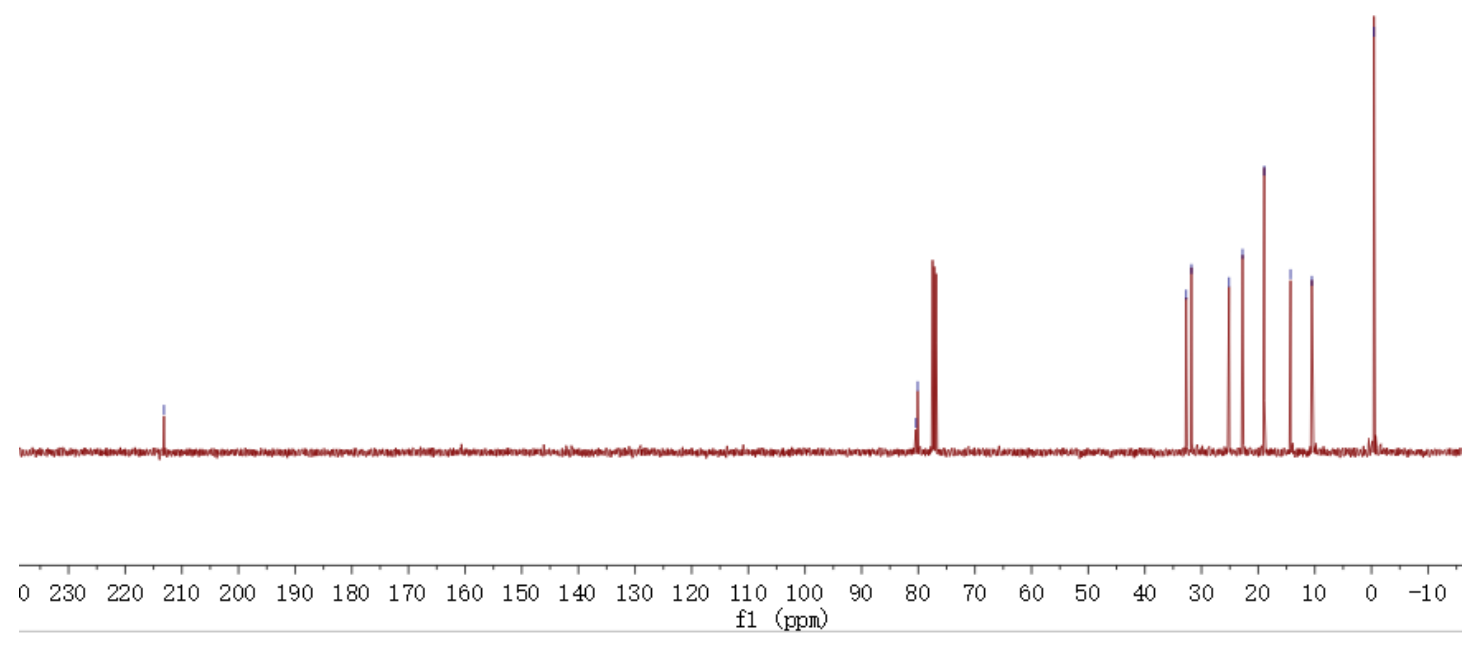




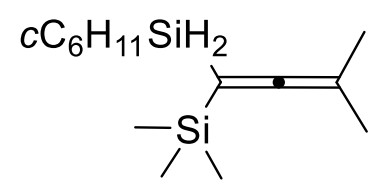

\section{${ }^{1} \mathrm{H}$ NMR spectrum in $\mathrm{CDCl}_{3}$}

$8 b$

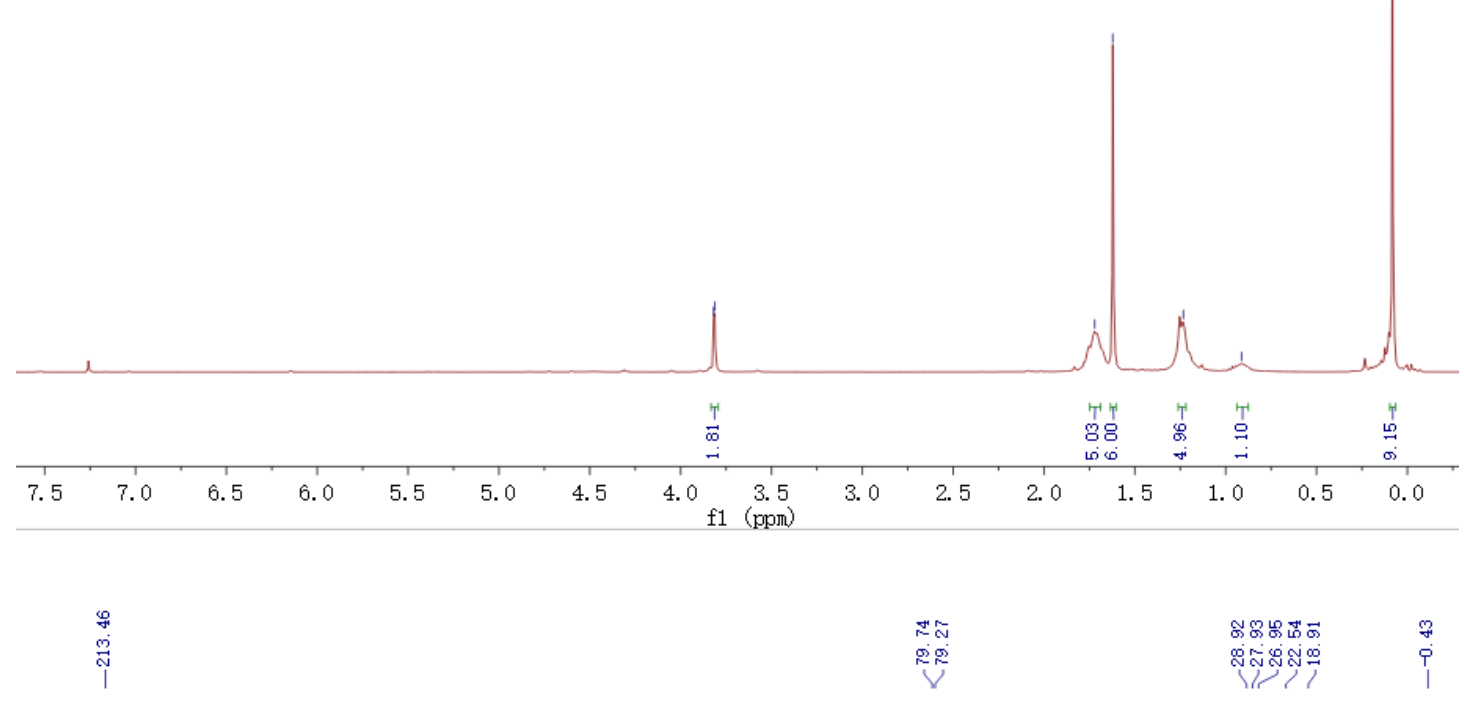

$c \mathrm{C}_{6} \mathrm{H}_{11} \mathrm{SiH}_{2}$

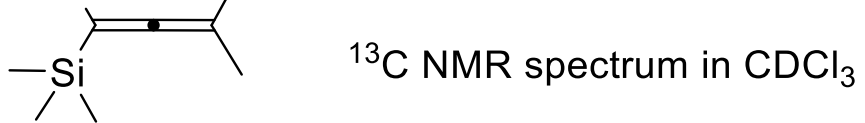

$8 b$

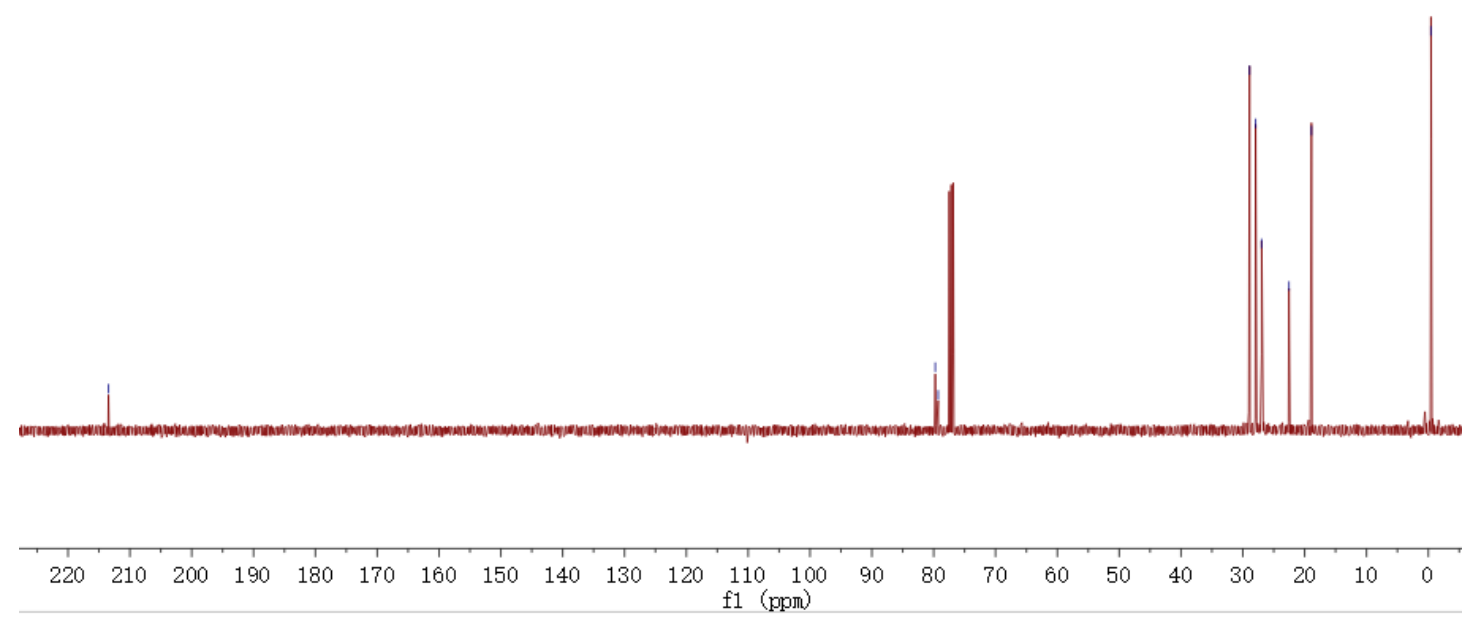



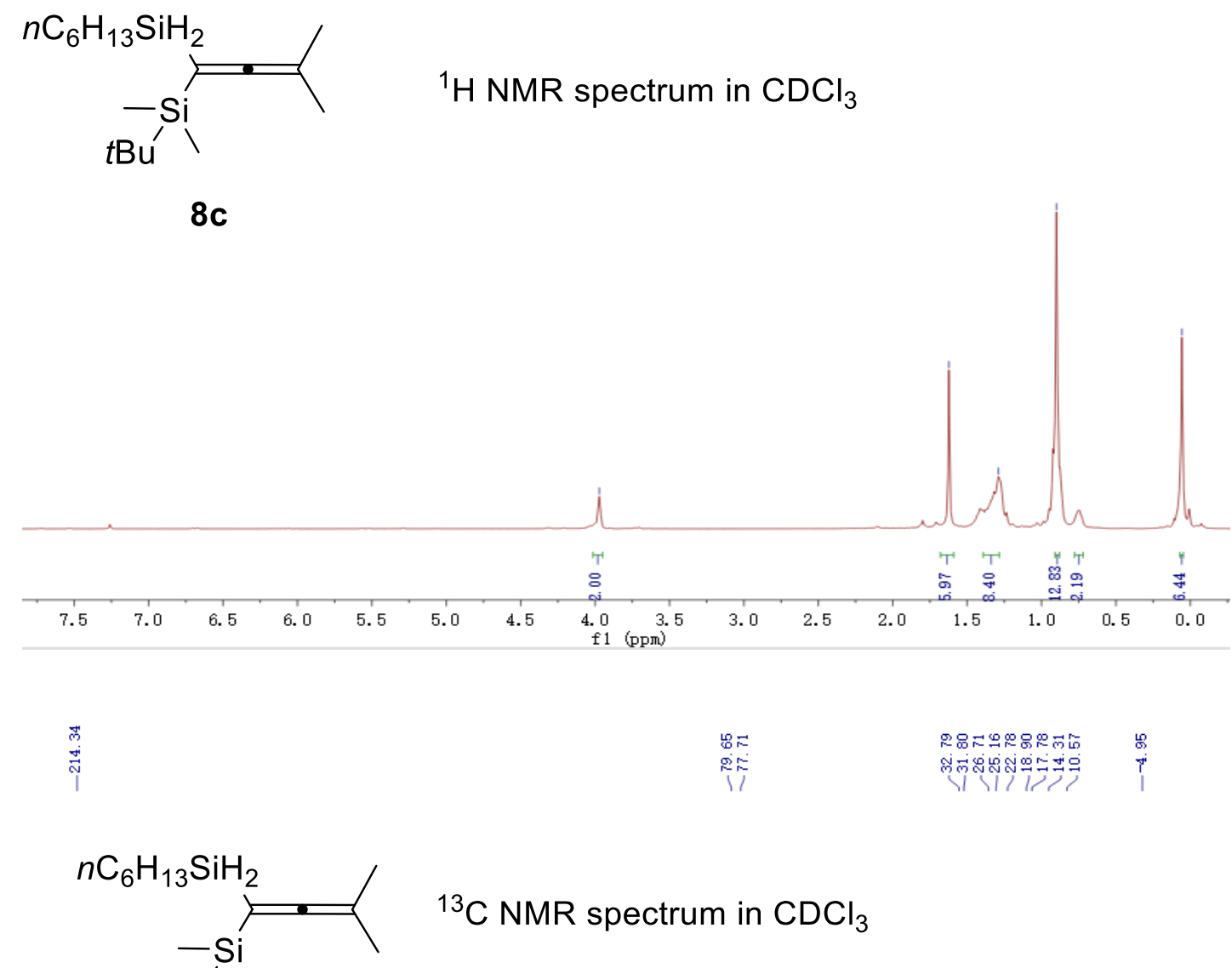

tBu

8c

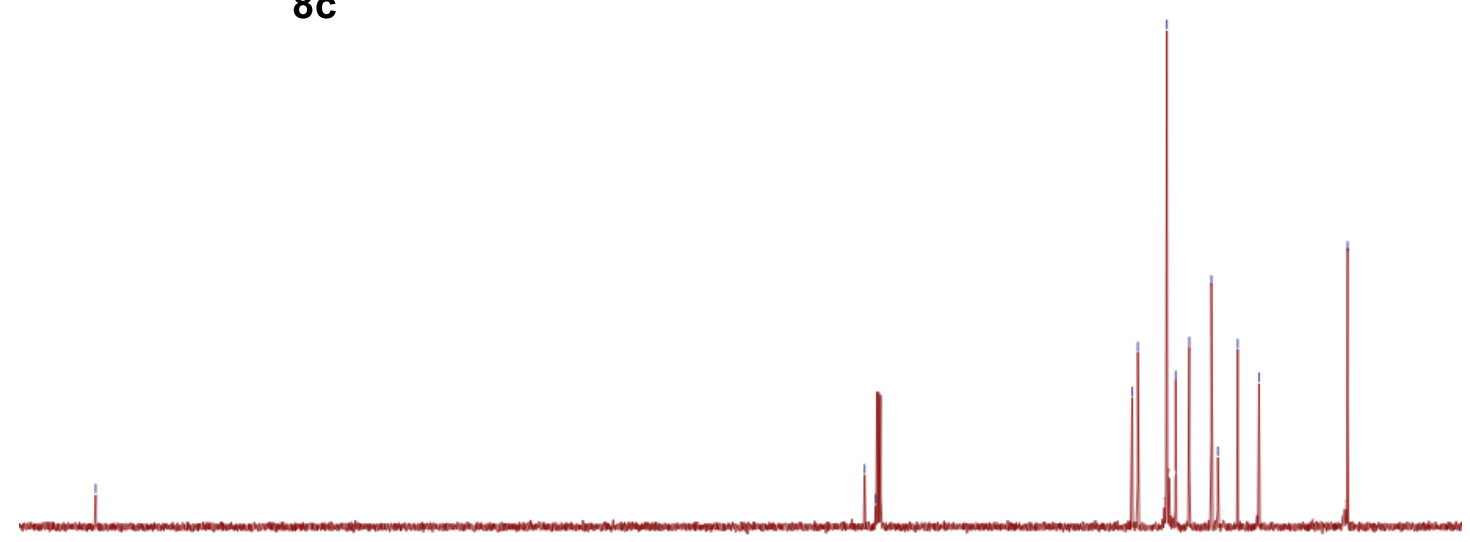

$\begin{array}{lllllllllllllllllllllllll}220 & 210 & 200 & 190 & 180 & 170 & 160 & 150 & 140 & 130 & 120 & 110 & 100 & 90 & 80 & 70 & 60 & 50 & 40 & 30 & 20 & 10 & 0 & -10 & -20\end{array}$ f1 (ppm) 


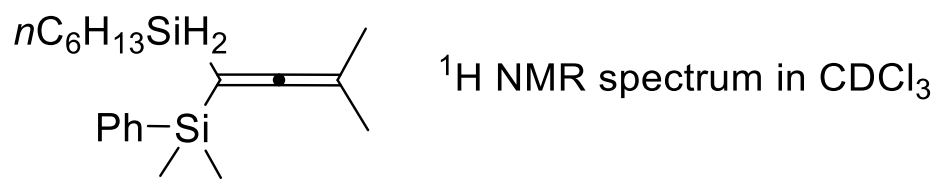

8d
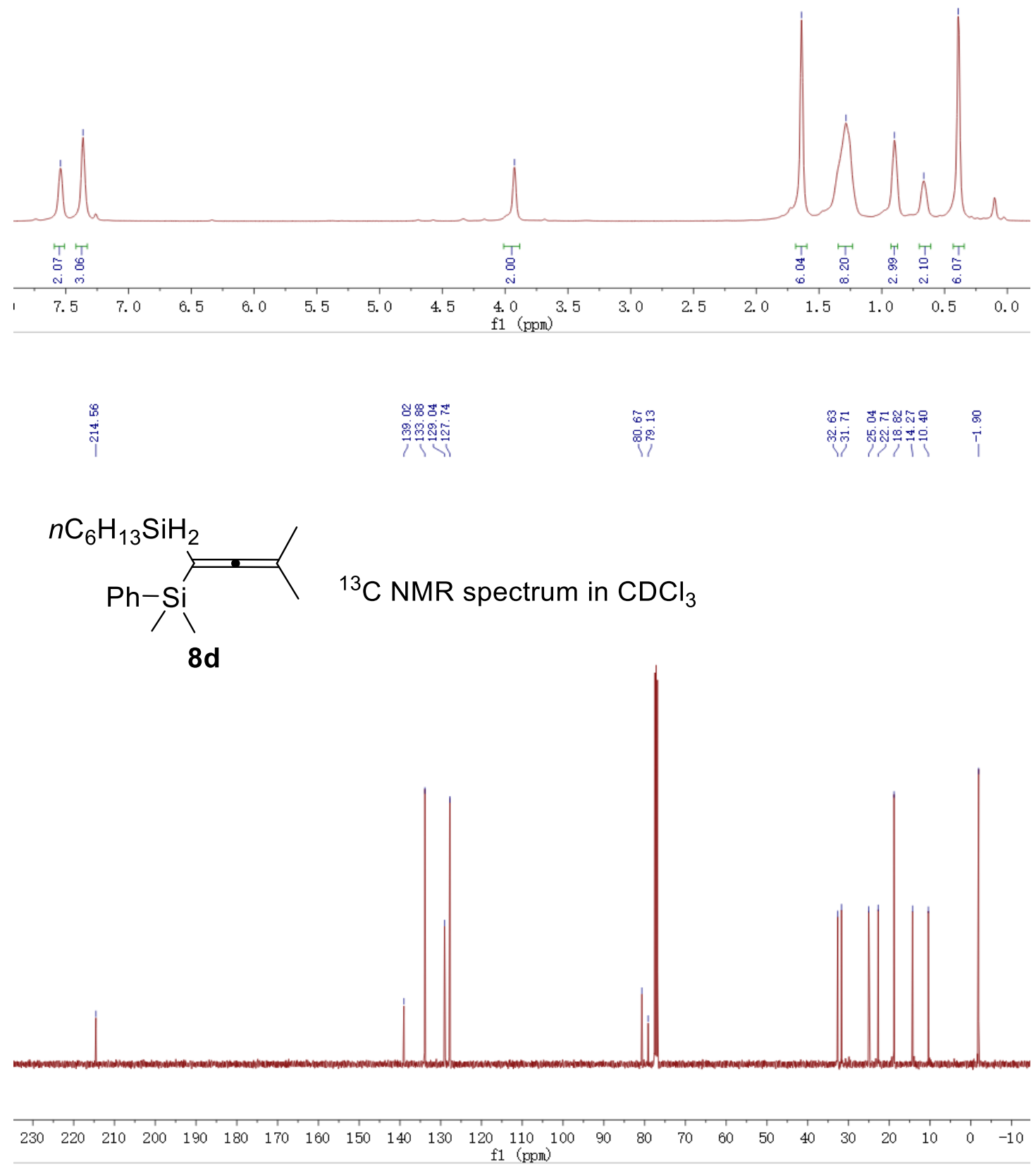

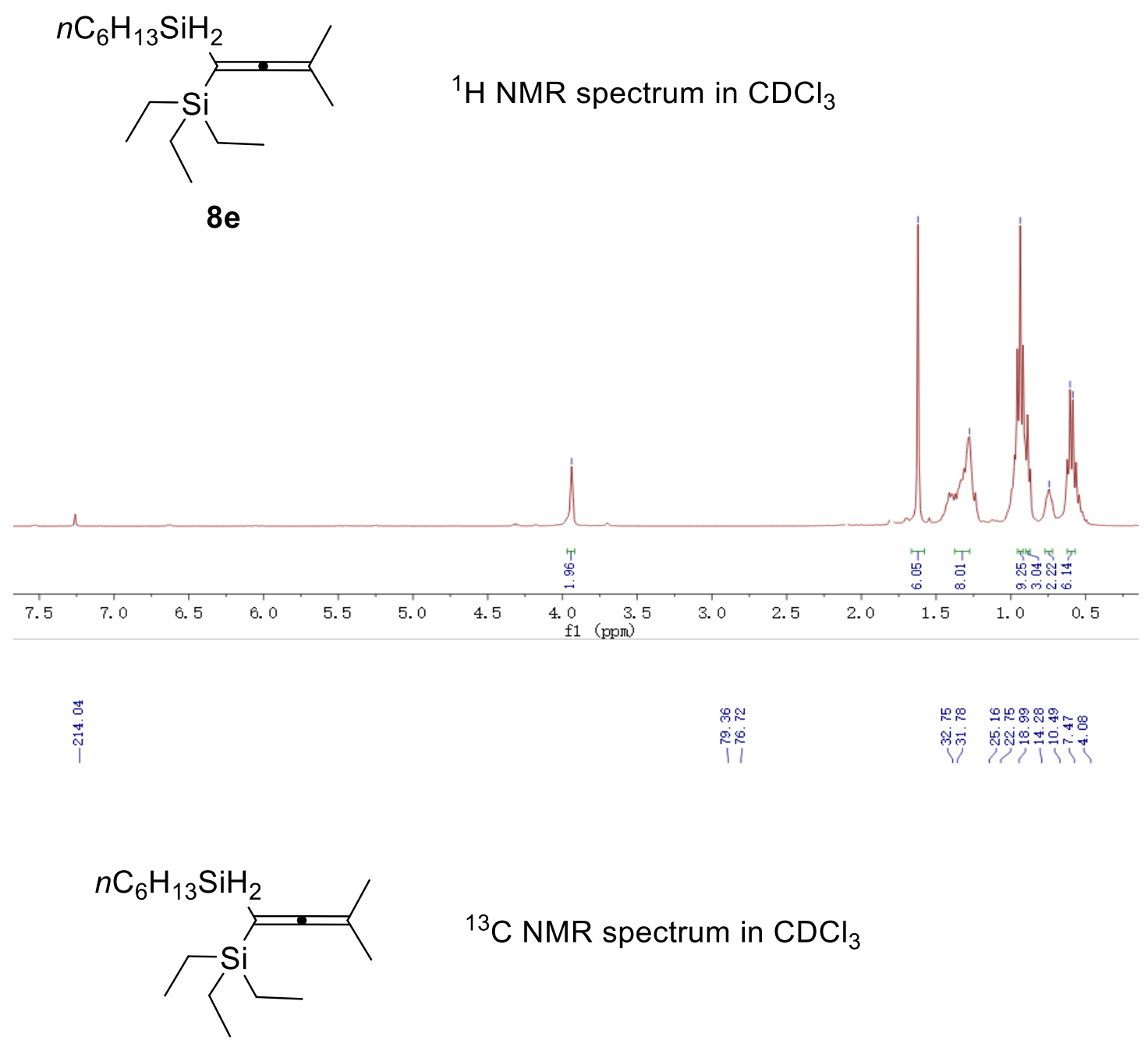

$8 e$

$\begin{array}{lllllllllllllllllllllll}220 & 210 & 200 & 190 & 180 & 170 & 160 & 150 & 140 & 130 & 120 & 110 & 100 & 90 & 80 & 70 & 60 & 50 & 40 & 30 & 20 & 10 & 0\end{array}$ 


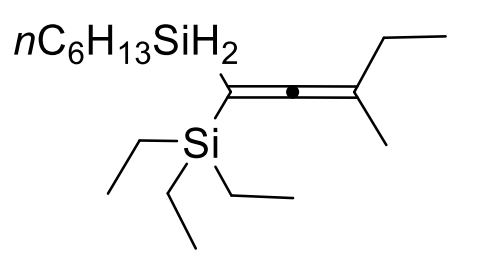

$8 f$

${ }^{1} \mathrm{H}$ NMR spectrum in $\mathrm{CDCl}_{3}$

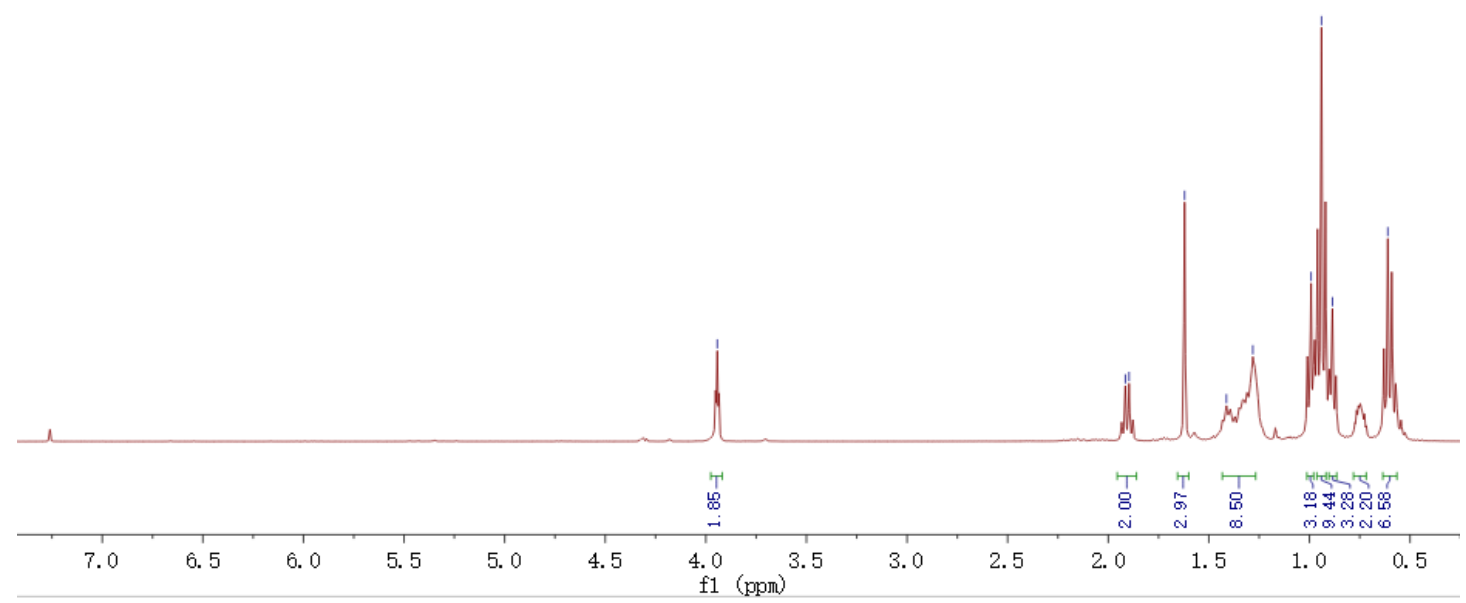

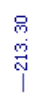

\begin{tabular}{ll}
$\vec{\infty}$ & \multirow{3}{*}{} \\
0 & 0 \\
0 & 0
\end{tabular}

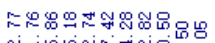

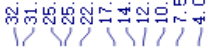
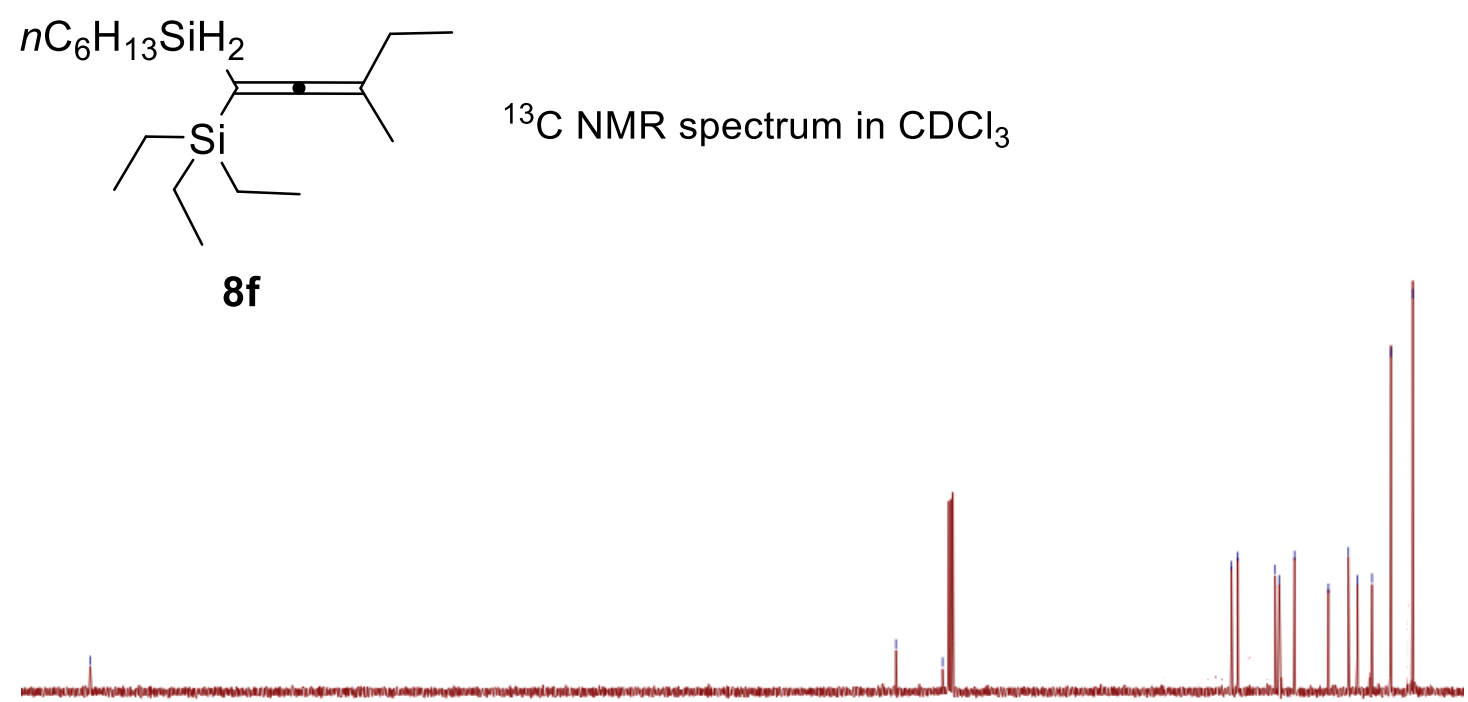

$\begin{array}{lllllllllllllllllllllllll}220 & 210 & 200 & 190 & 180 & 170 & 160 & 150 & 140 & 130 & 120 & 110 & 100 & 90 & 80 & 70 & 60 & 50 & 40 & 30 & 20 & 10 & 0\end{array}$ f1 (ppm) 


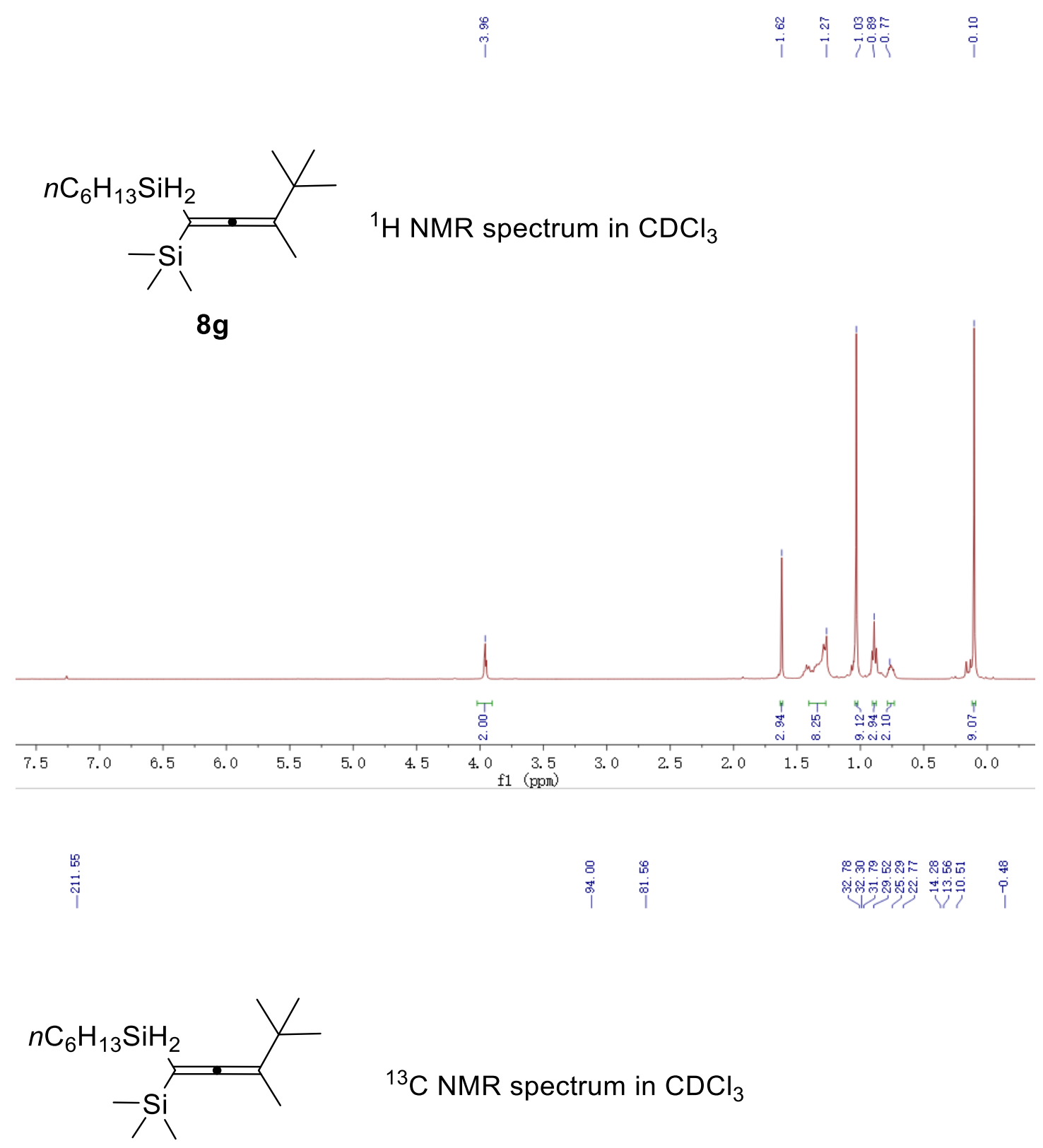

$8 \mathrm{~g}$

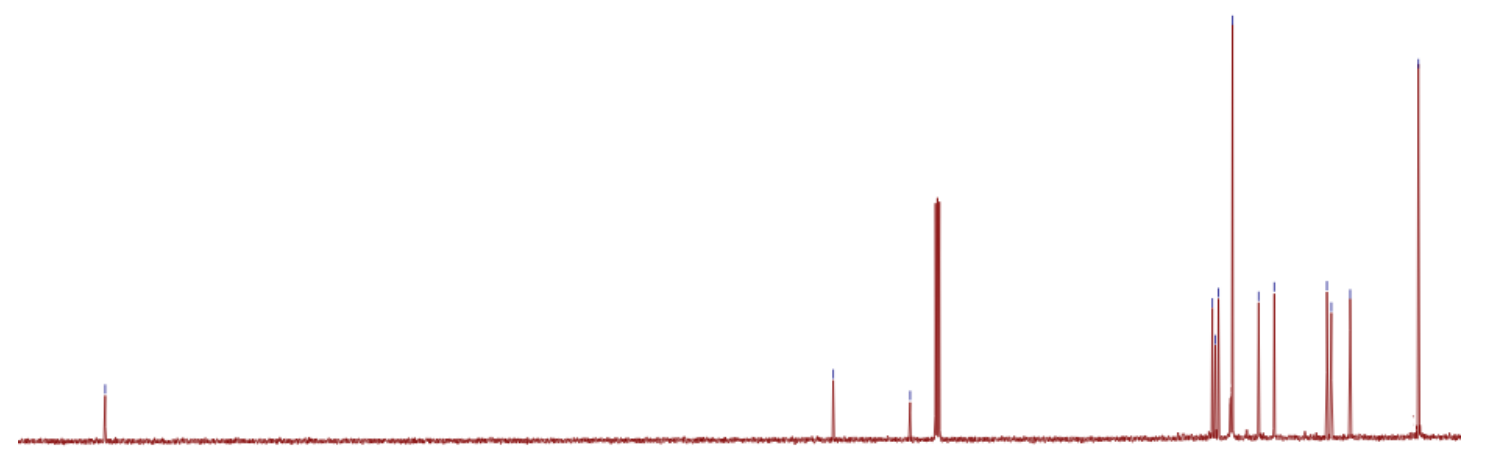

$\begin{array}{lllllllllllllllllllllll}220 & 210 & 200 & 190 & 180 & 170 & 160 & 150 & 140 & 130 & 120 & 110 & 100 & 90 & 80 & 70 & 60 & 50 & 40 & 30 & 20 & 10 & 0\end{array}$ f1 (ppm) 


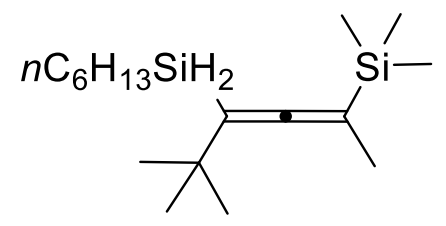

$8 h$
${ }^{1} \mathrm{H}$ NMR spectrum in $\mathrm{CDCl}_{3}$

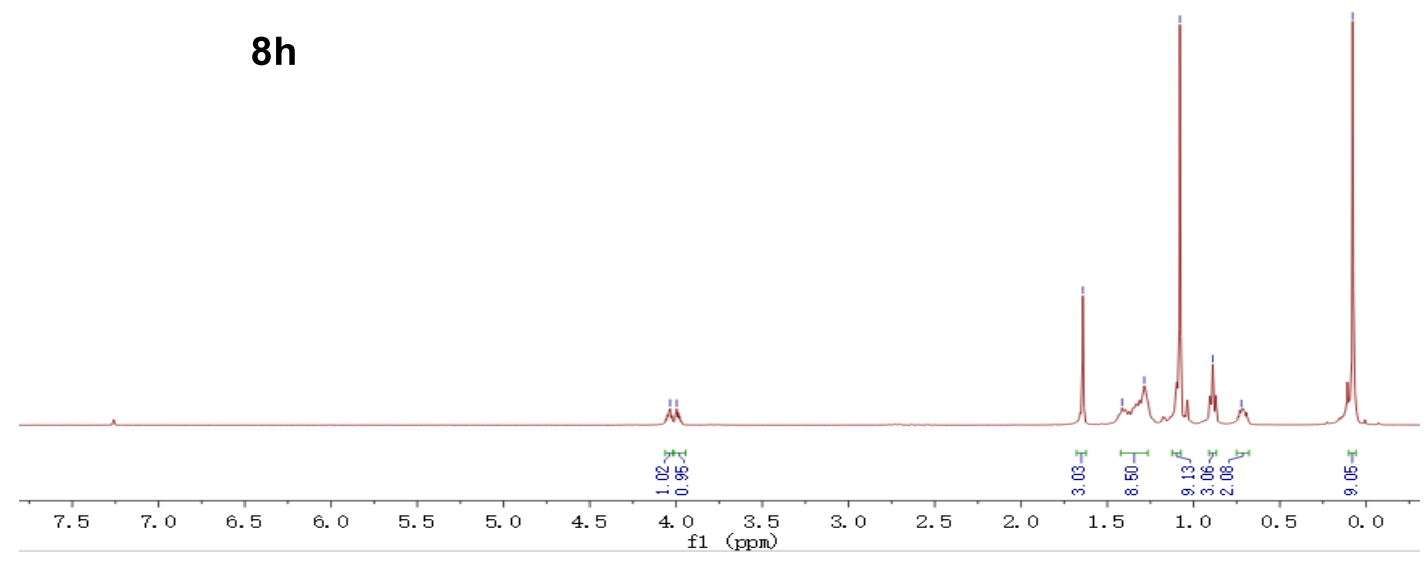

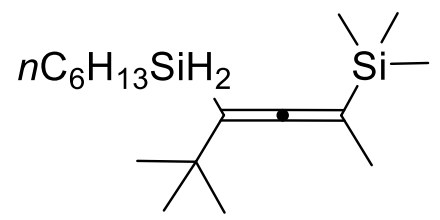

${ }^{13} \mathrm{C}$ NMR spectrum in $\mathrm{CDCl}_{3}$

$8 h$

$\begin{array}{rrrrrrrrrrrrr}220 & 210 & 200 & 190 & 180 & 170 & 160 & 150 & 140 & 130 & 120 & 110 & 100 \\ \mathrm{f1} & (\mathrm{ppm})\end{array}$ 


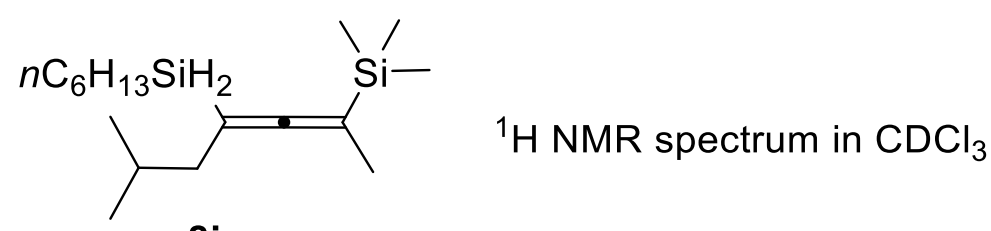

$8 i$

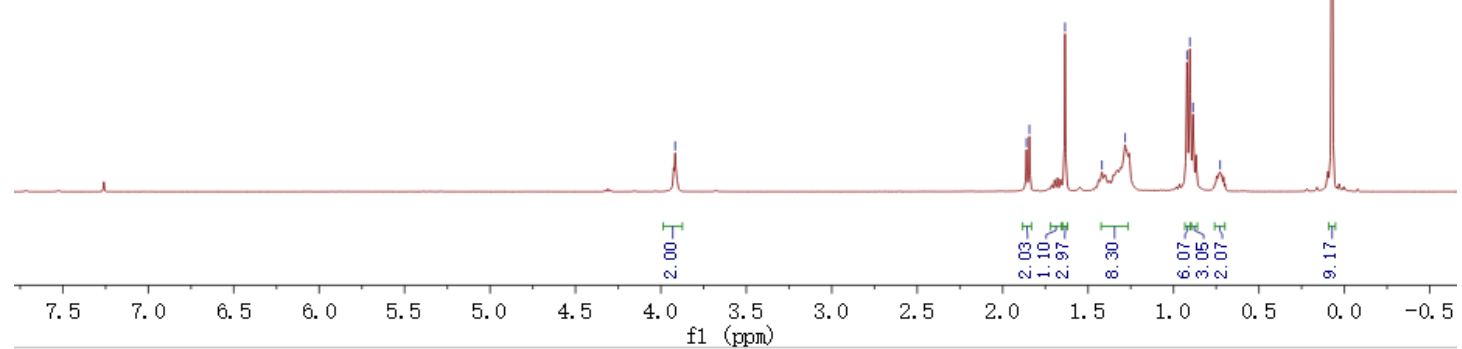

吕

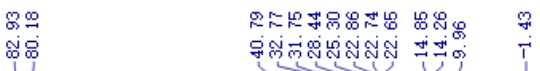

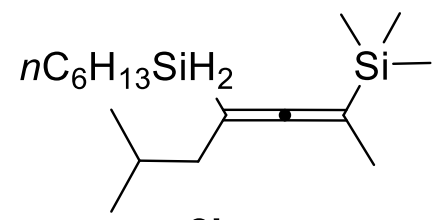

${ }^{13} \mathrm{C}$ NMR spectrum in $\mathrm{CDCl}_{3}$

$8 i$

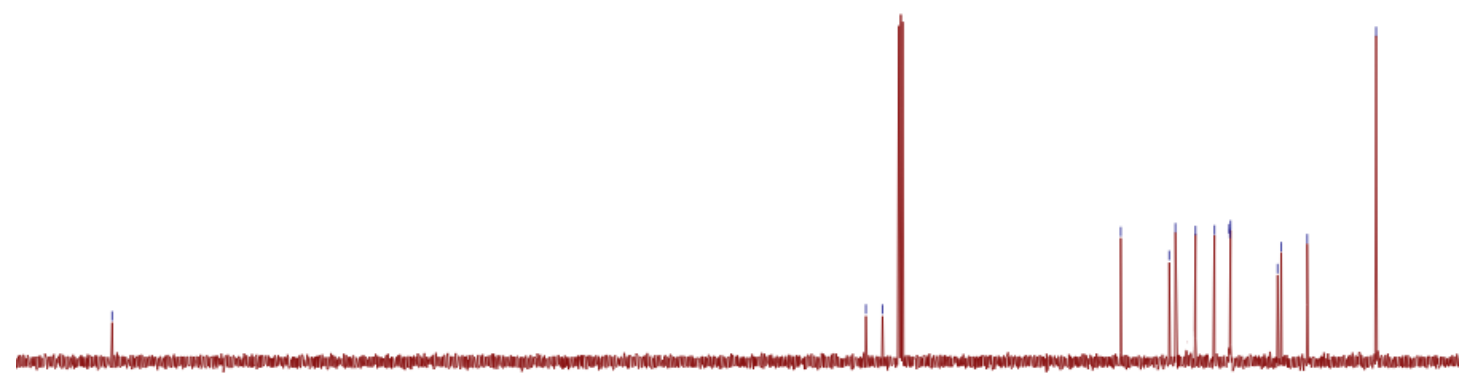

$\begin{array}{llllllllllllllllllllllll}220 & 210 & 200 & 190 & 180 & 170 & 160 & 150 & 140 & 130 & 120 & 110 & 100 & 90 & 80 & 70 & 60 & 50 & 40 & 30 & 20 & 10 & 0 & -10\end{array}$ f1 (ppm) 


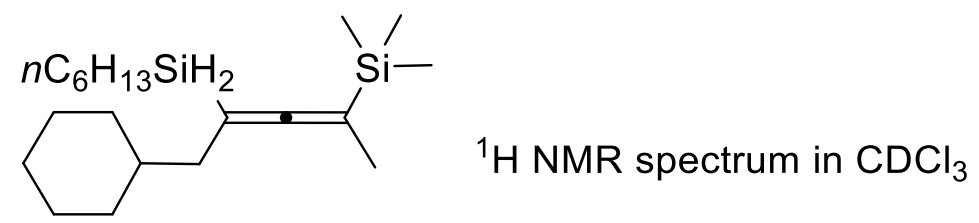

8j
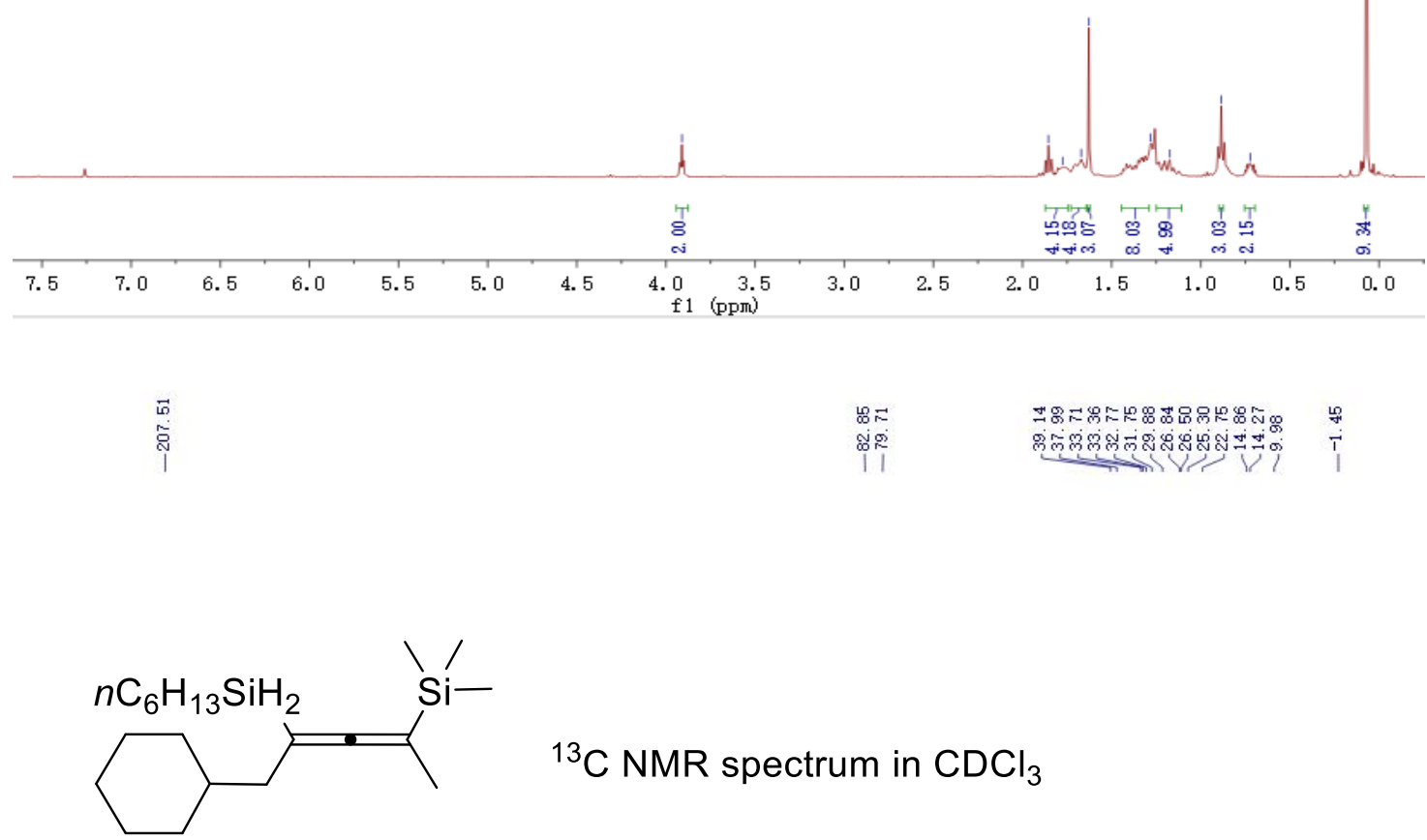

${ }^{13} \mathrm{C}$ NMR spectrum in $\mathrm{CDCl}_{3}$

8j

$\begin{array}{llllllllllllll}230 & 220 & 210 & 200 & 190 & 180 & 170 & 160 & 150 & 140 & 130 & 120 & 110 & 100 \\ \mathrm{f1} & \text { (ppm) }\end{array}$ 

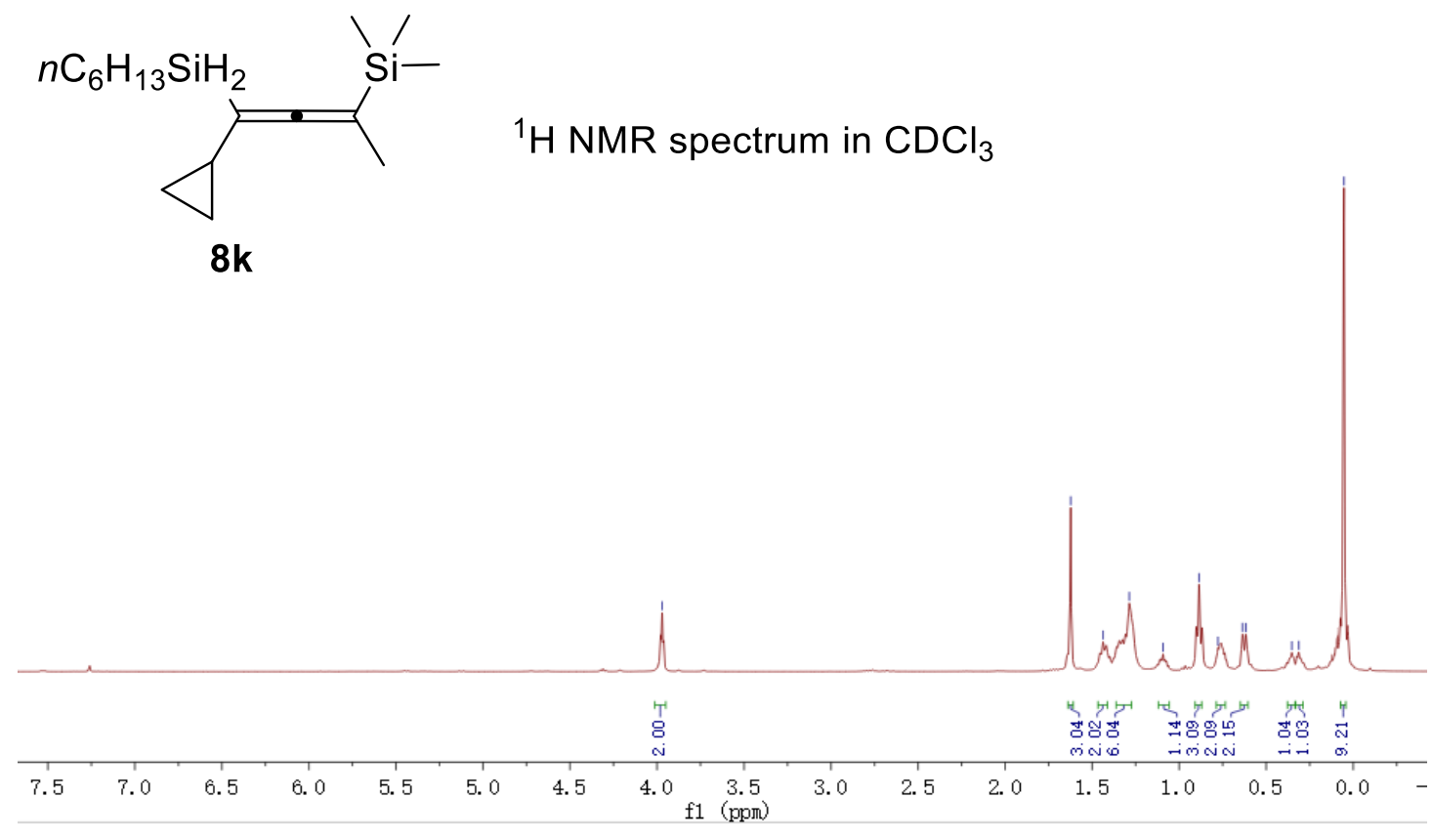



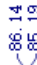

유

लूल

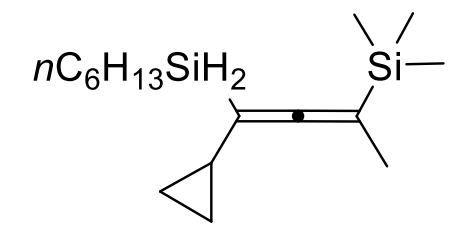

${ }^{13} \mathrm{C}$ NMR spectrum in $\mathrm{CDCl}_{3}$

8k

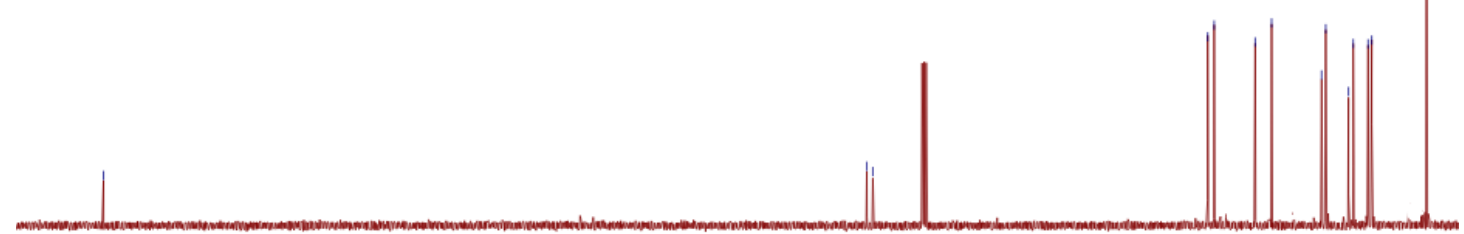

$\begin{array}{llllllllllll}210 & 200 & 190 & 180 & 170 & 160 & 150 & 140 & 130 & 120 & 110 & 100\end{array}$ 

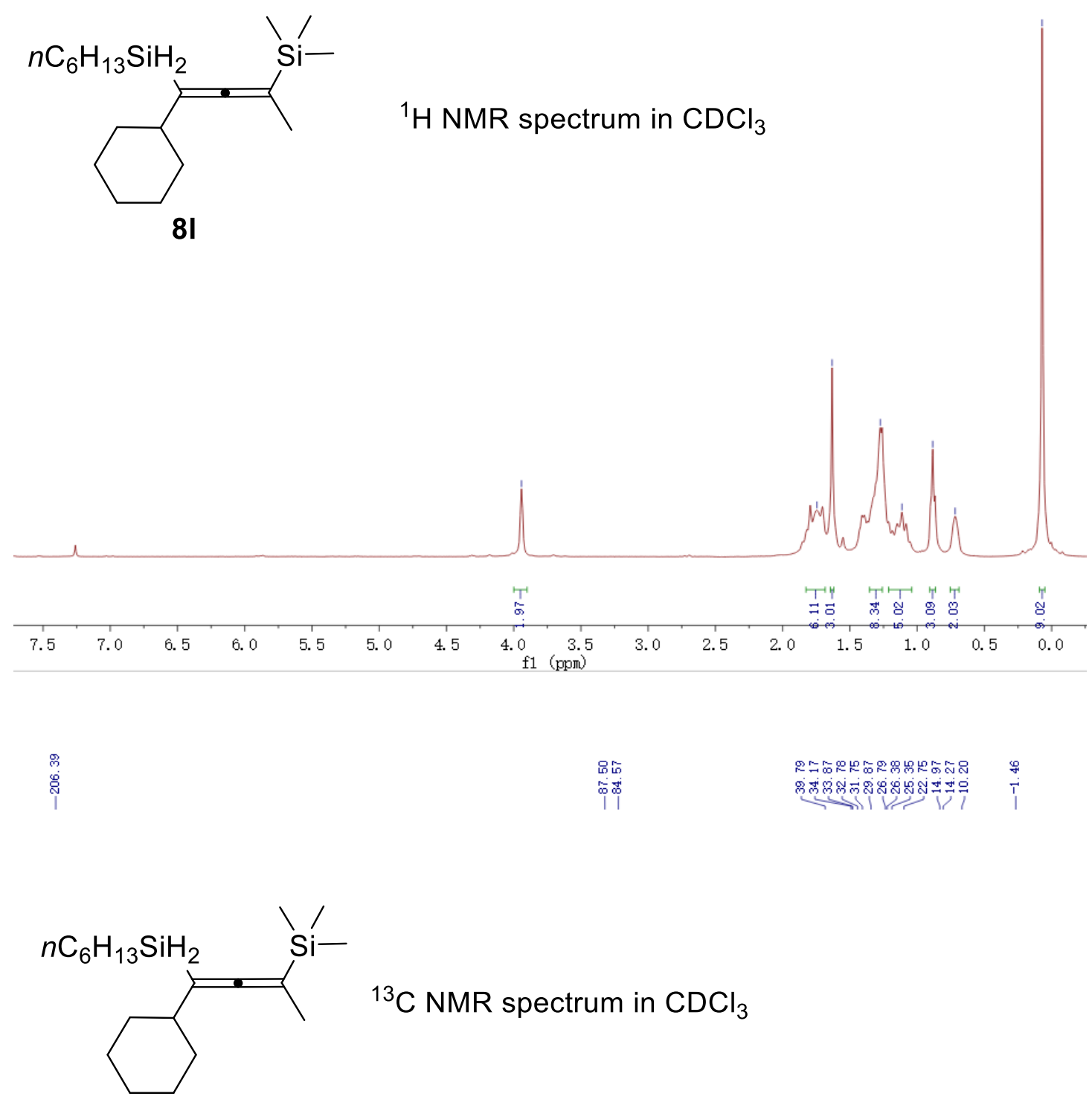

81

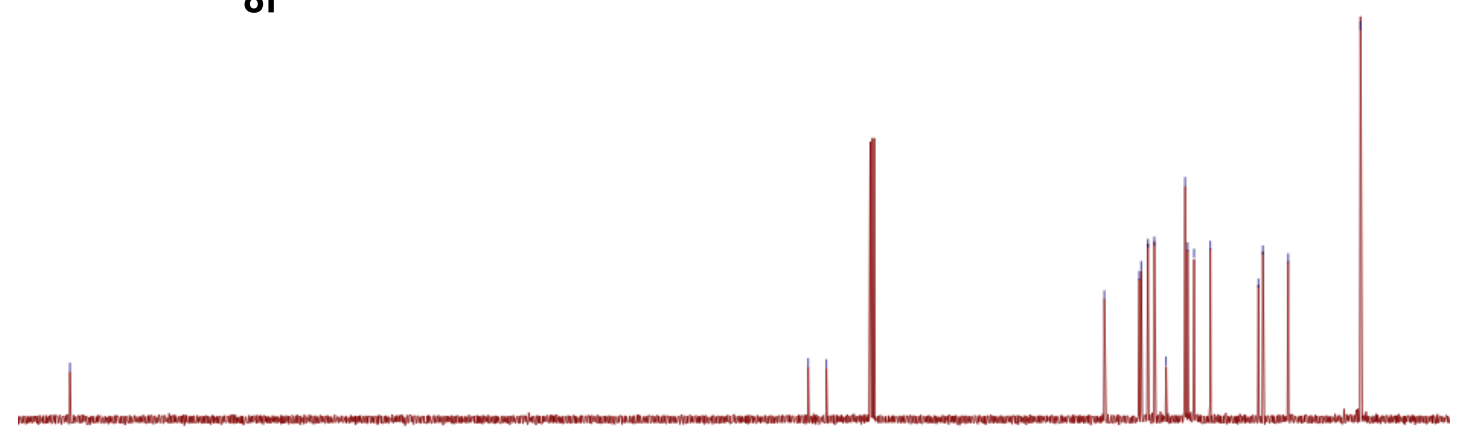

$\begin{array}{llllllllllllllllllllllllllll}210 & 200 & 190 & 180 & 170 & 160 & 150 & 140 & 130 & 120 & 110 & 100 & 90 & 80 & 70 & 60 & 50 & 40 & 30 & 20 & 10 & 0 & -10\end{array}$ 


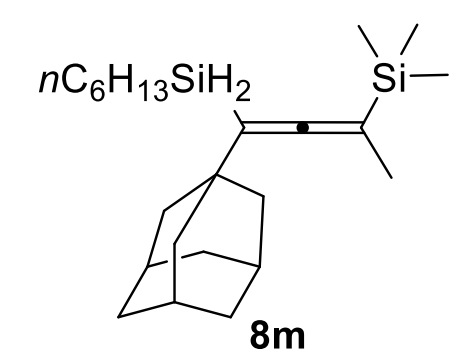

${ }^{1} \mathrm{H}$ NMR spectrum in $\mathrm{CDCl}_{3}$
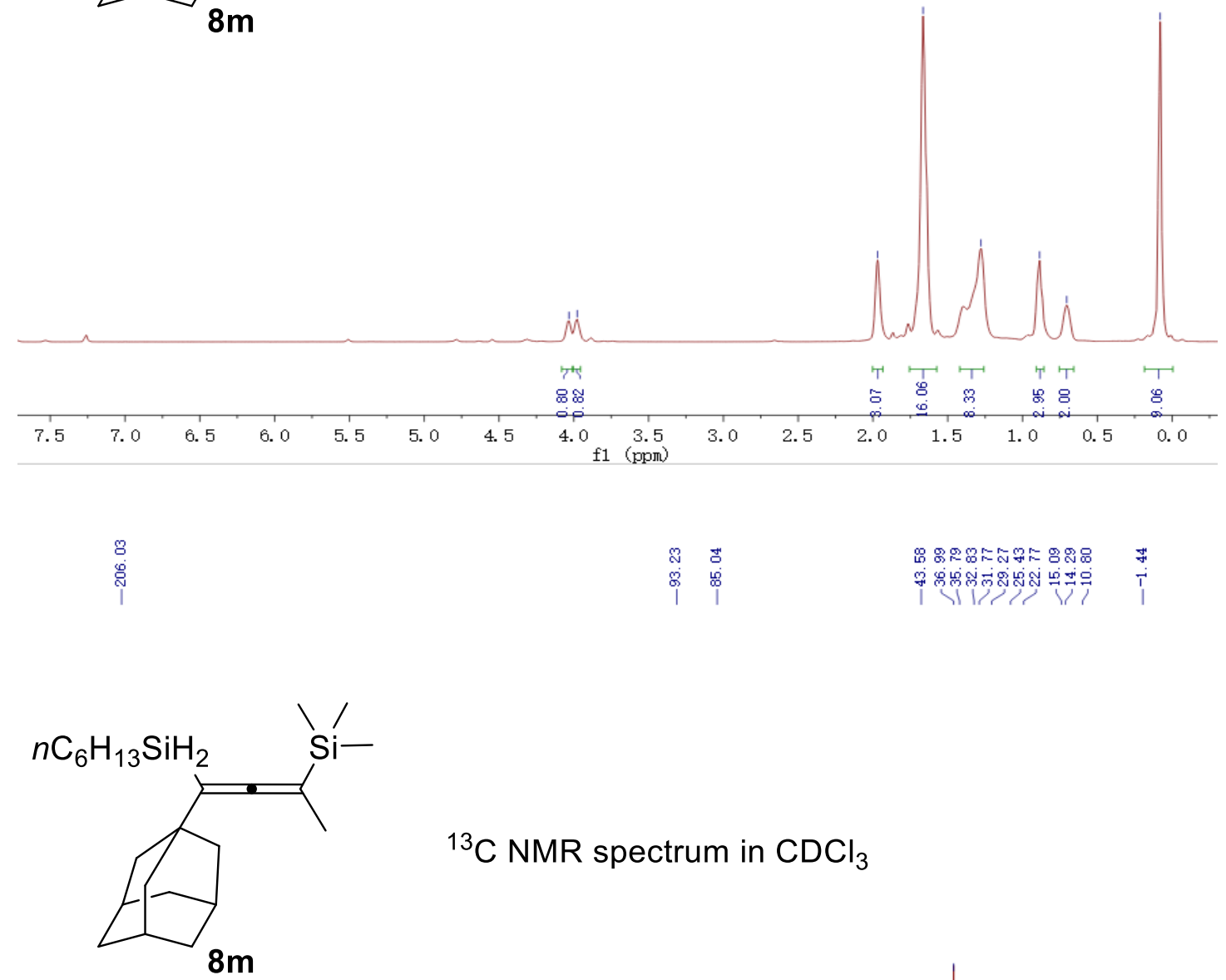

${ }^{13} \mathrm{C}$ NMR spectrum in $\mathrm{CDCl}_{3}$

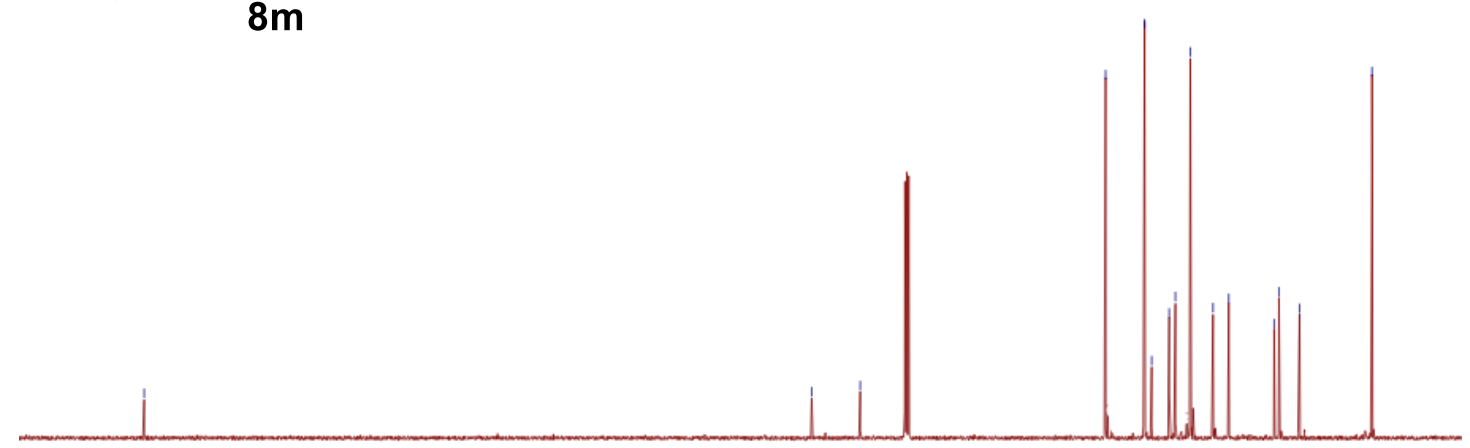

$\begin{array}{lllllllllllllllllllllllll}220 & 210 & 200 & 190 & 180 & 170 & 160 & 150 & 140 & 130 & 120 & 110 & 100 & 90 & 80 & 70 & 60 & 50 & 40 & 30 & 20 & 10 & 0 & -10\end{array}$ 


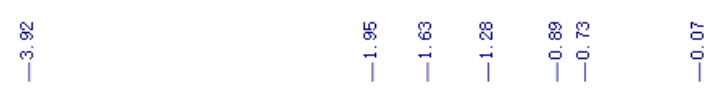

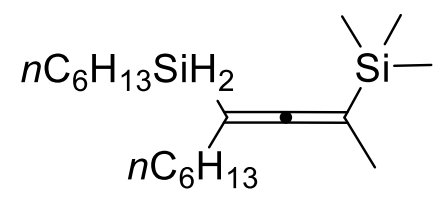

${ }^{1} \mathrm{H}$ NMR spectrum in $\mathrm{CDCl}_{3}$

$8 n$

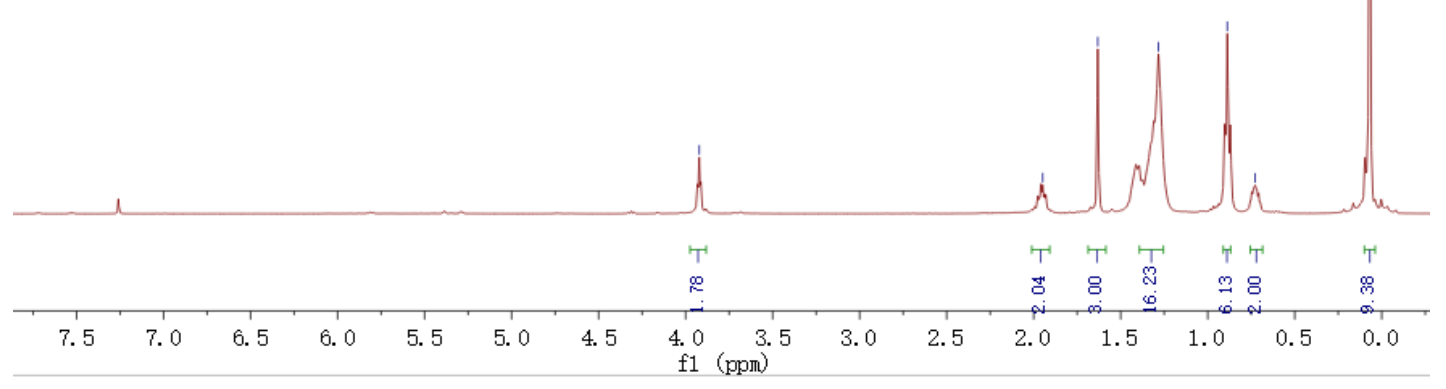

$\stackrel{8}{\stackrel{8}{\circ}}$

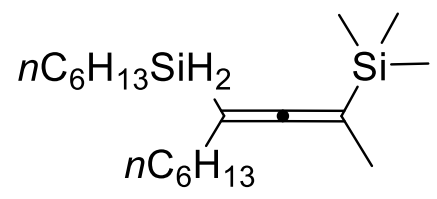

${ }^{13} \mathrm{C} \mathrm{NMR}$ spectrum in $\mathrm{CDCl}_{3}$

$8 n$

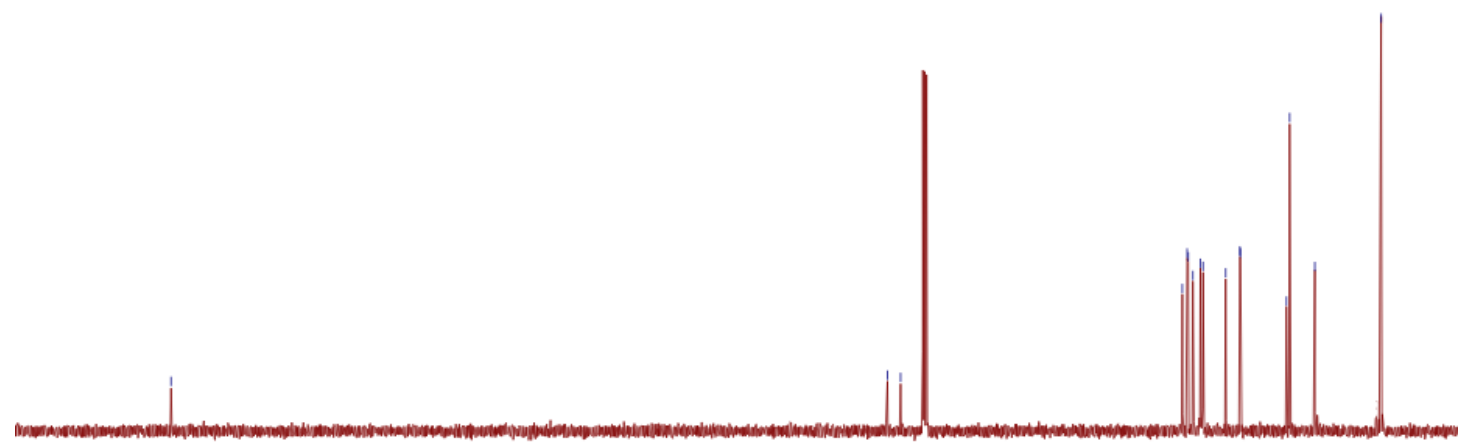

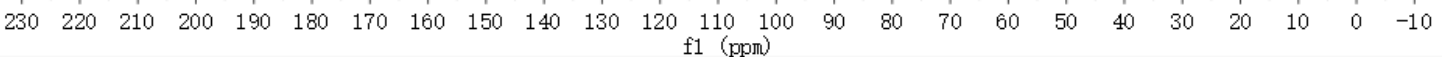



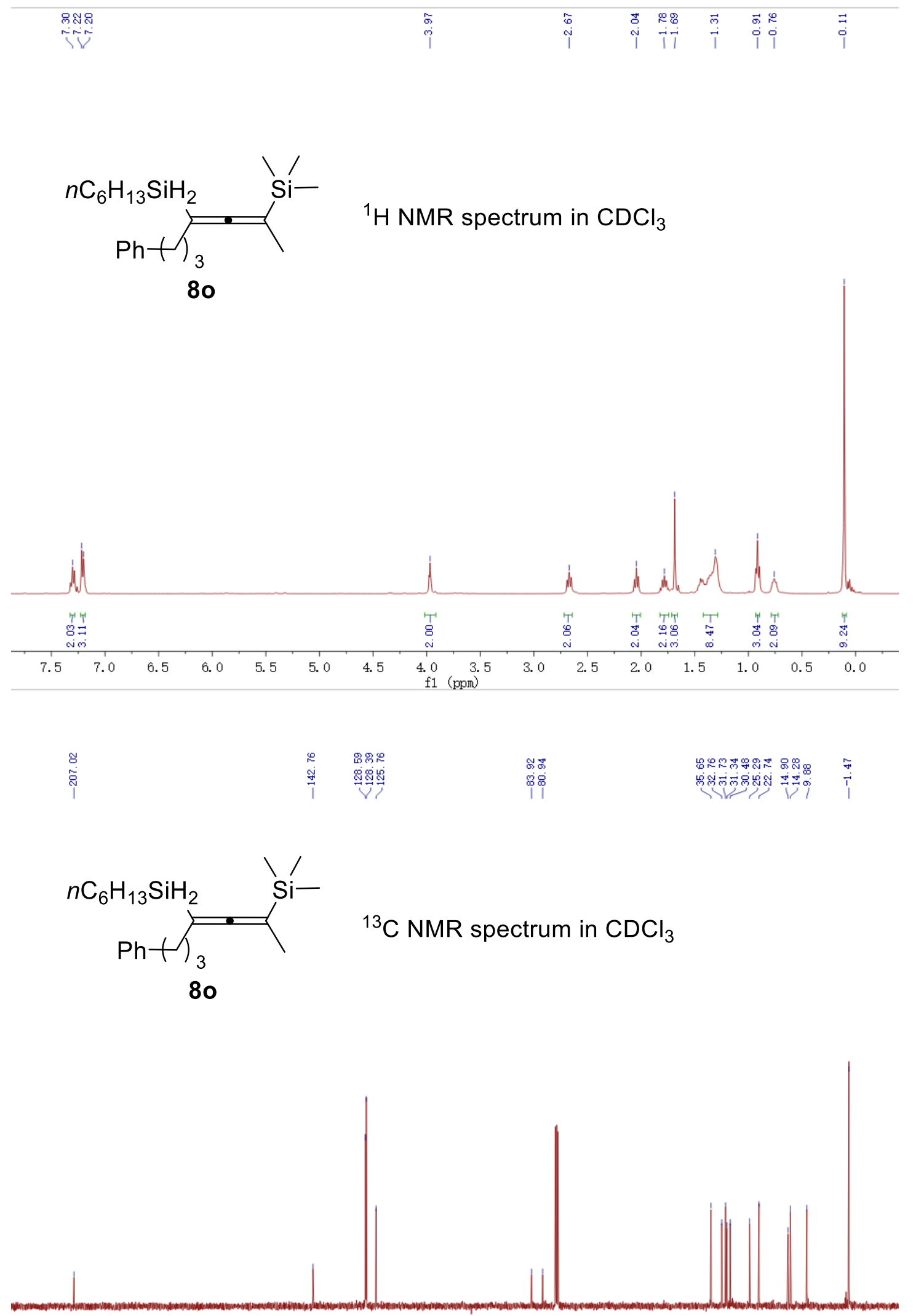

$\begin{array}{llllllllllllllllllllllllllllll}220 & 210 & 200 & 190 & 180 & 170 & 160 & 150 & 140 & 130 & 120 & 110 & 100 & 90 & 80 & 70 & 60 & 50 & 40 & 30 & 20 & 10 & 0 & -10\end{array}$ f1 (ppr) 


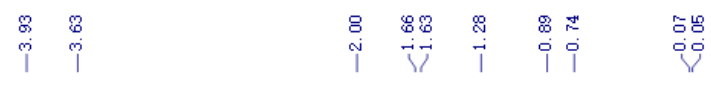
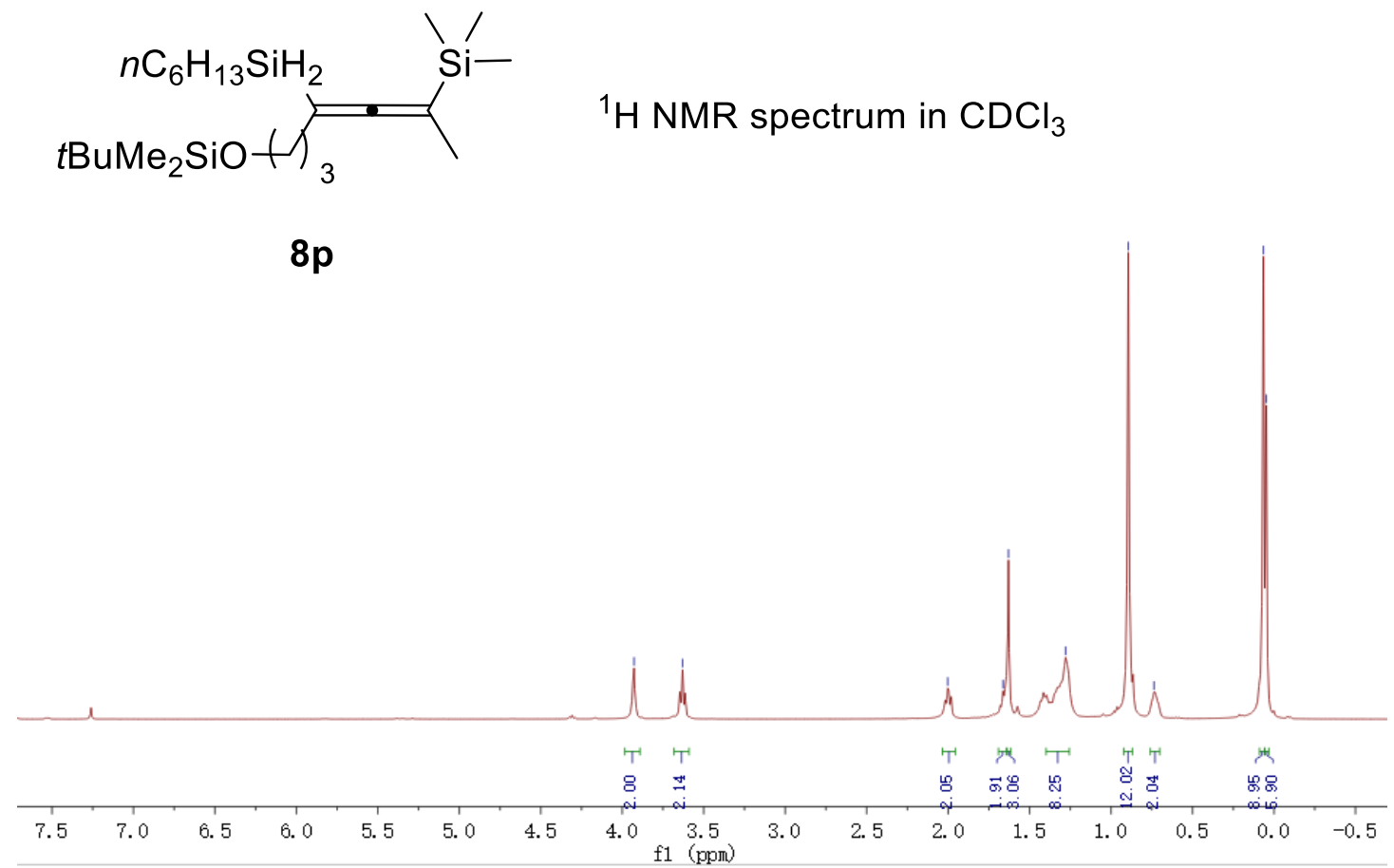

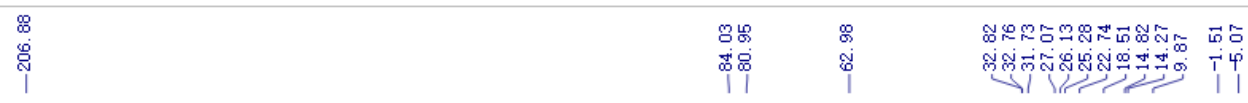

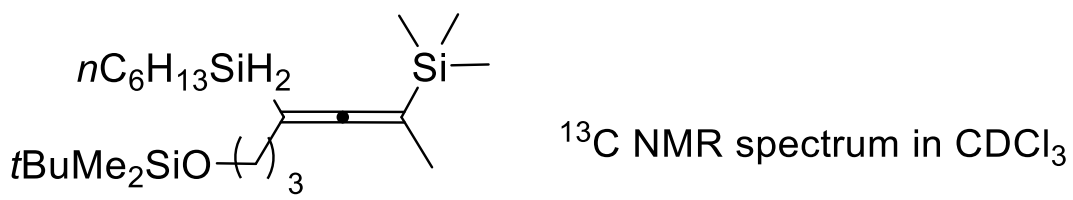

$8 p$

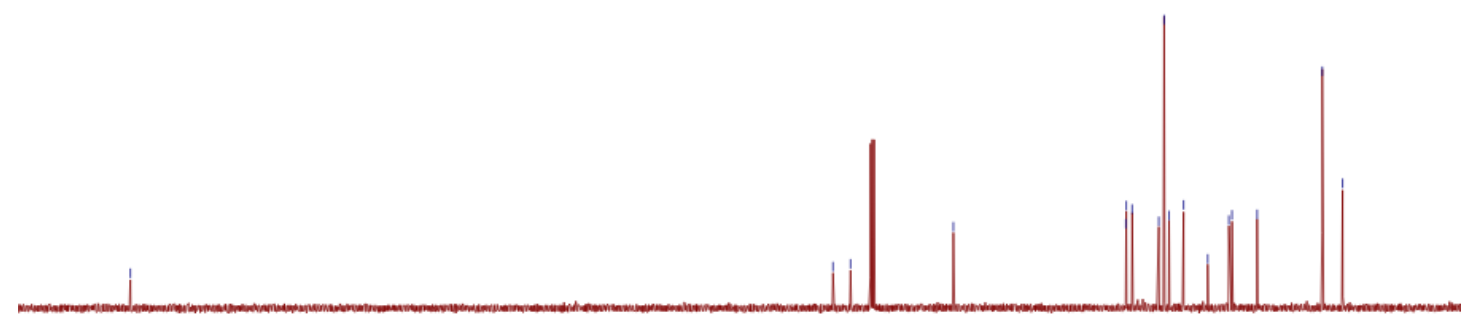

$\begin{array}{lllllllllllllllllllllllll}220 & 210 & 200 & 190 & 180 & 170 & 160 & 150 & 140 & 130 & 120 & 110 & 100 & 90 & 80 & 70 & 60 & 50 & 40 & 30 & 20 & 10 & 0 & -10 & -20\end{array}$ f1 (ppm) 


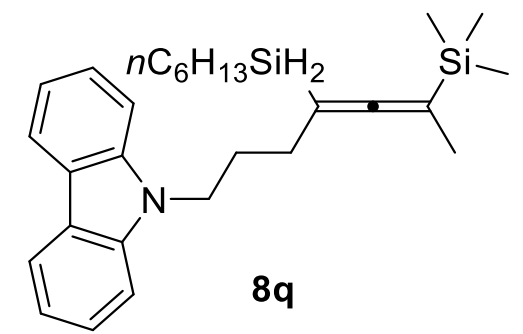

${ }^{1} \mathrm{H}$ NMR spectrum in $\mathrm{CDCl}_{3}$

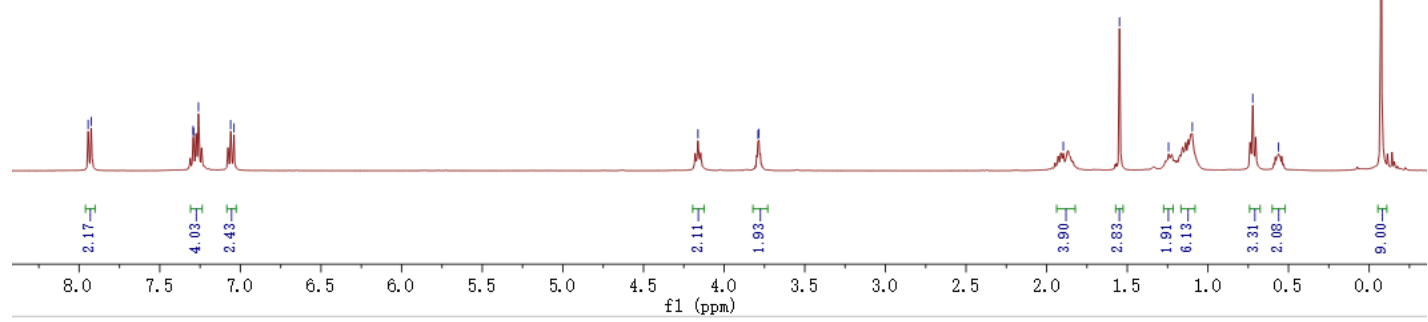

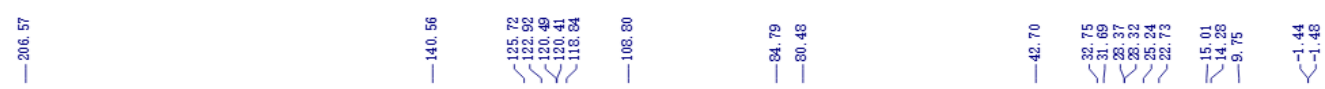

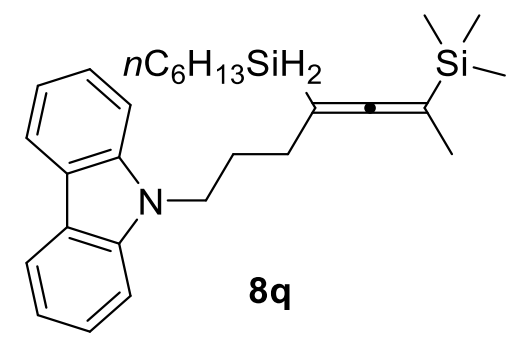

${ }^{13} \mathrm{C}$ NMR spectrum in $\mathrm{CDCl}_{3}$

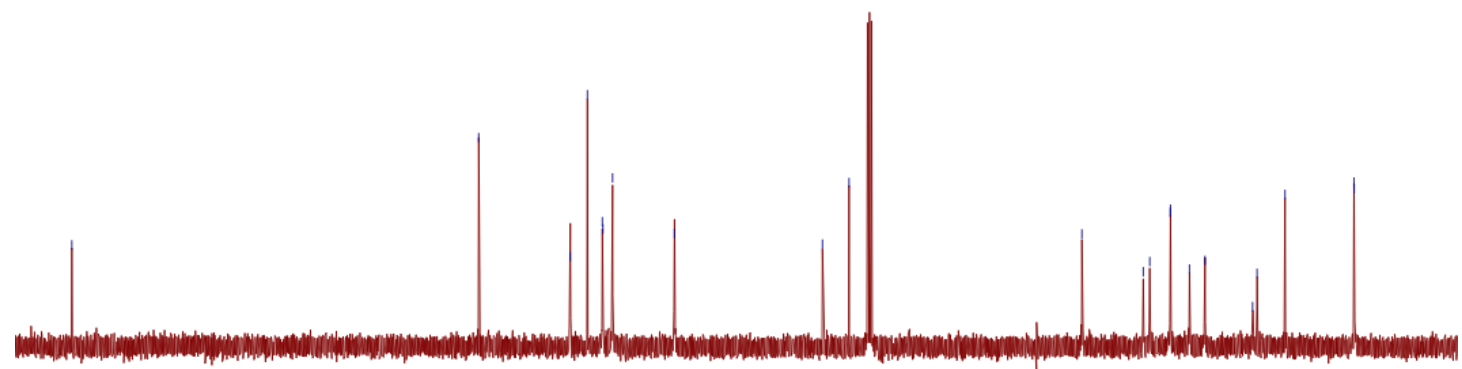

$\begin{array}{llllllllllllll}210 & 200 & 190 & 180 & 170 & 160 & 150 & 140 & 130 & 120 & 110 & 100 \\ \mathrm{f} 1 & (\mathrm{ppm})\end{array}$ 

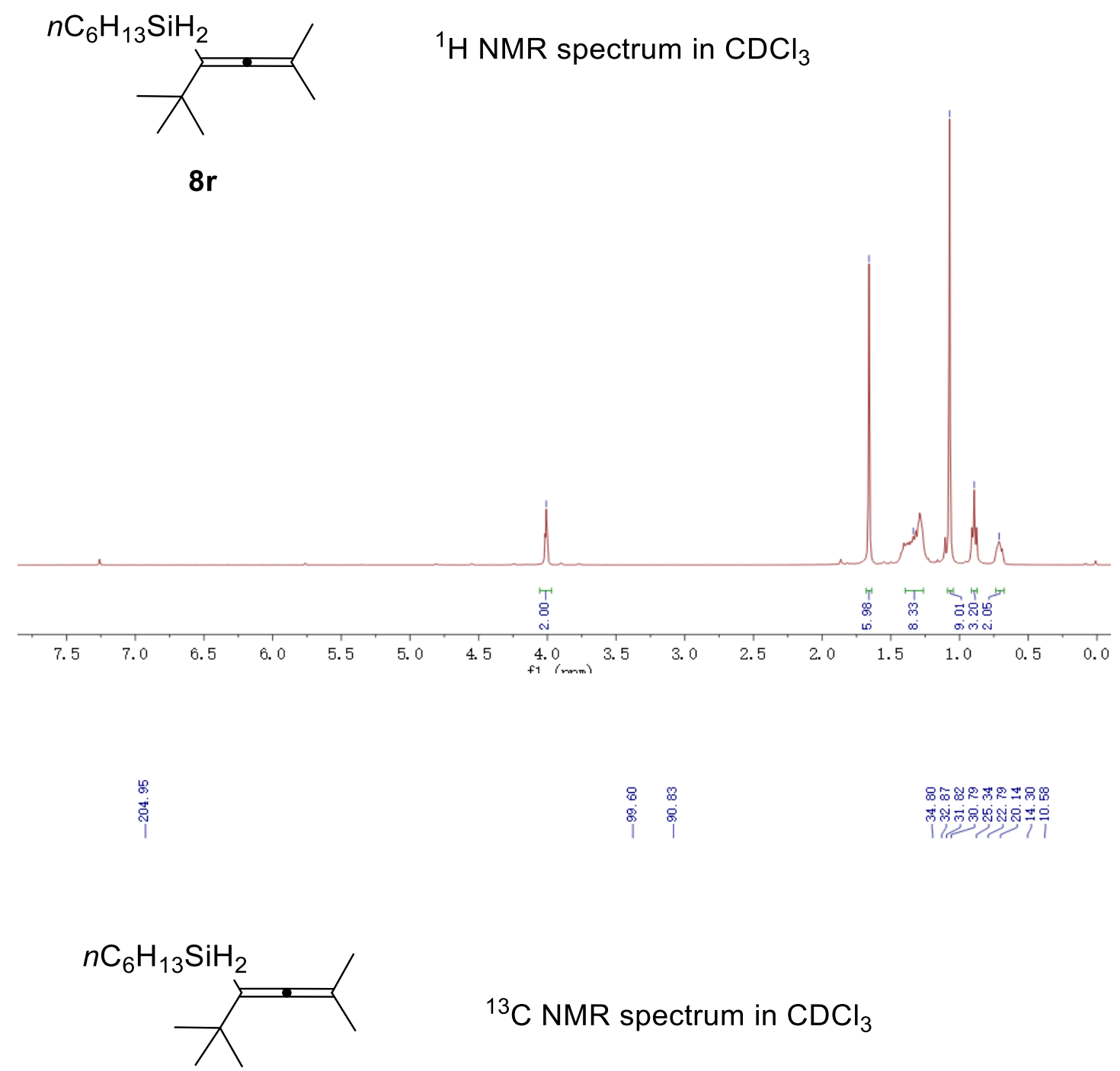

${ }^{13} \mathrm{C}$ NMR spectrum in $\mathrm{CDCl}_{3}$

$8 r$

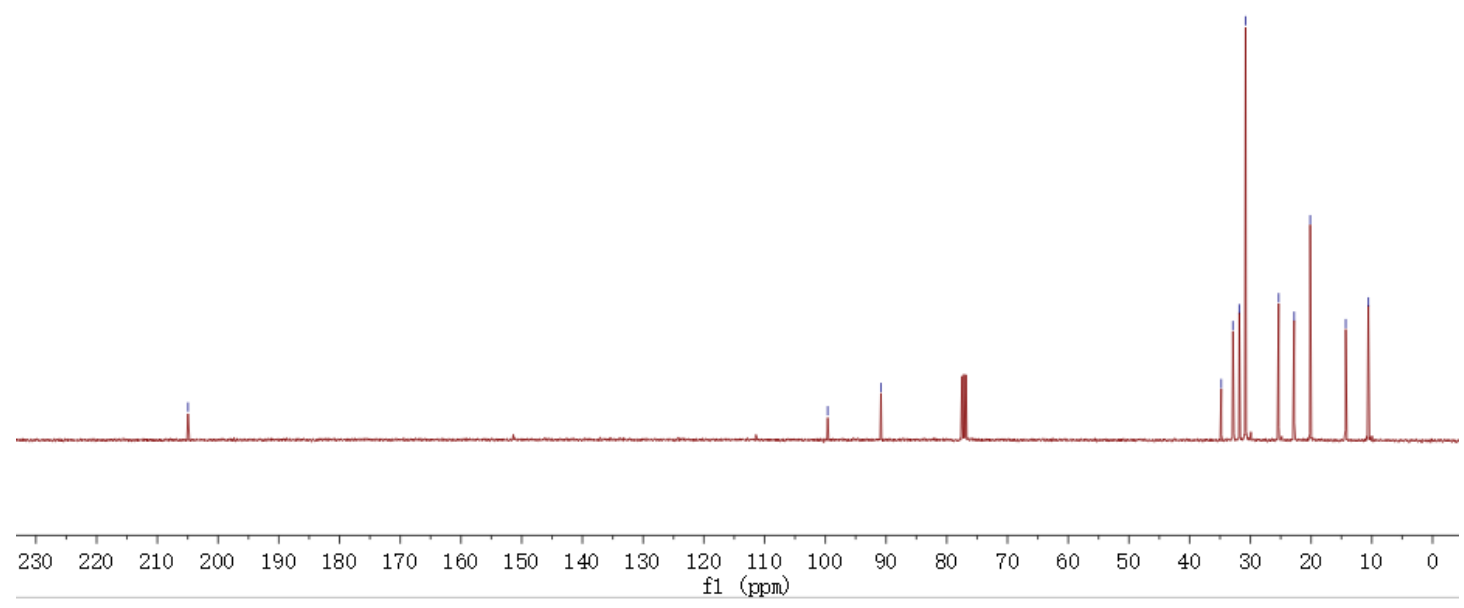


$n \mathrm{C}_{6} \mathrm{H}_{13} \mathrm{SiH}_{2}$

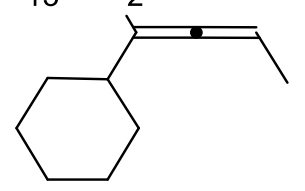

${ }^{1} \mathrm{H}$ NMR spectrum in $\mathrm{CDCl}_{3}$

$8 s$

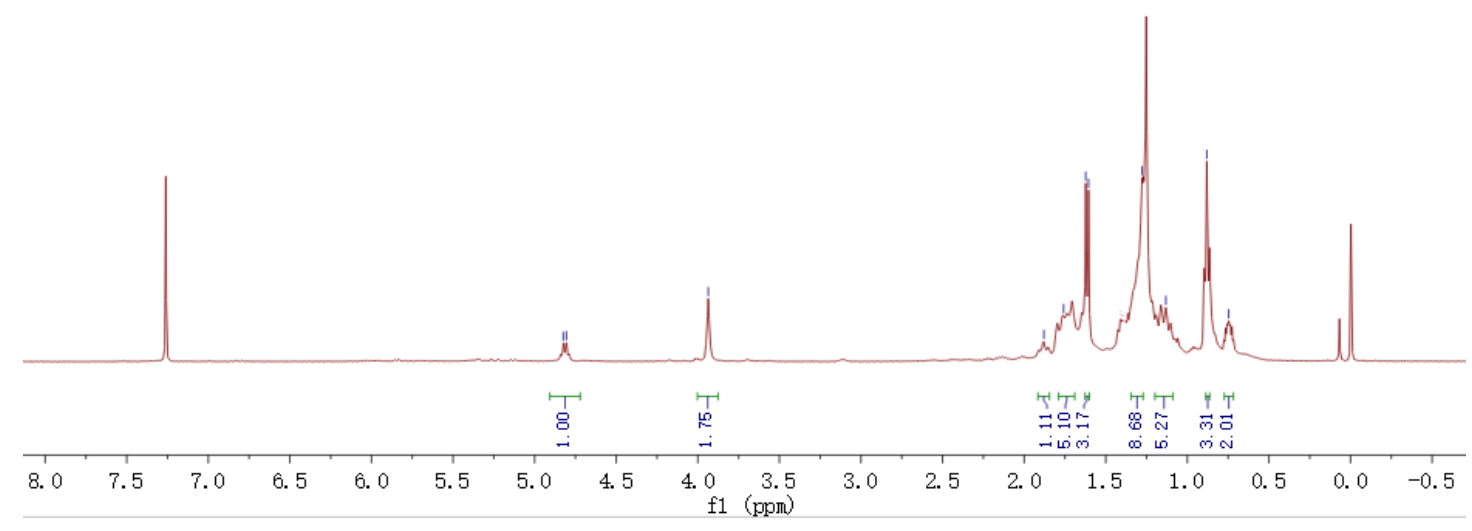

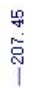

$n \mathrm{C}_{6} \mathrm{H}_{13} \mathrm{SiH}_{2}$

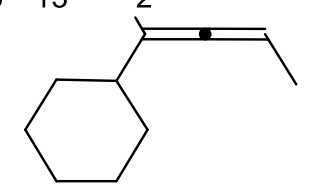

${ }^{13} \mathrm{C}$ NMR spectrum in $\mathrm{CDCl}_{3}$

$8 s$

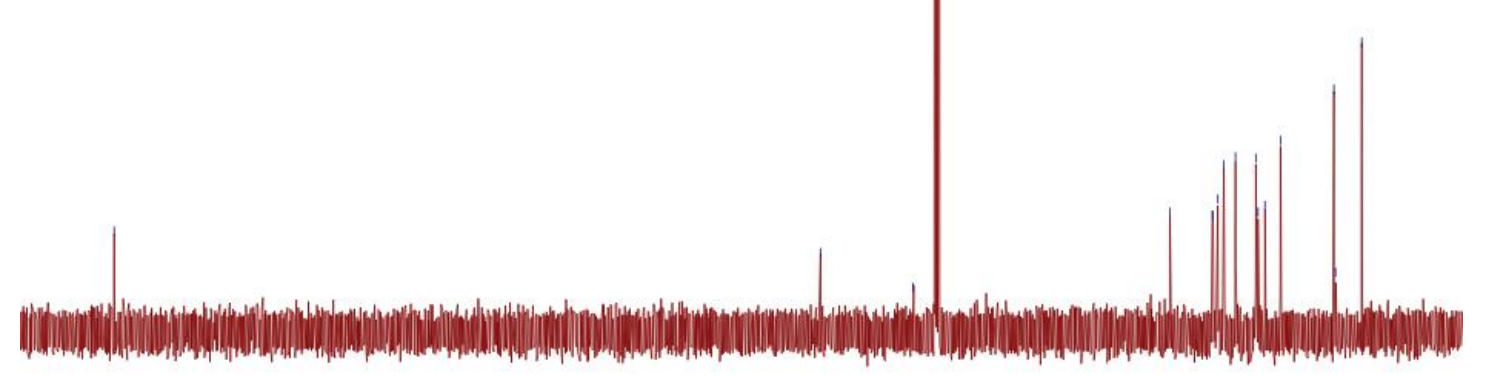

$\begin{array}{lllllllllllllllllllllllllllllllll}220 & 210 & 200 & 190 & 180 & 170 & 160 & 150 & 140 & 130 & 120 & 110 & 100 & 90 & 80 & 70 & 60 & 50 & 40 & 30 & 20 & 10 & 0\end{array}$ f1 (ppm) 


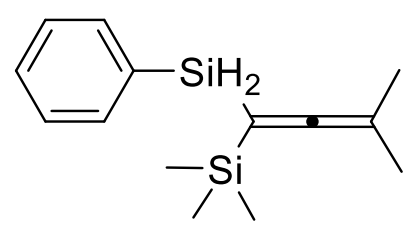

${ }^{1} \mathrm{H}$ NMR spectrum in $\mathrm{CDCl}_{3}$

$8 t$
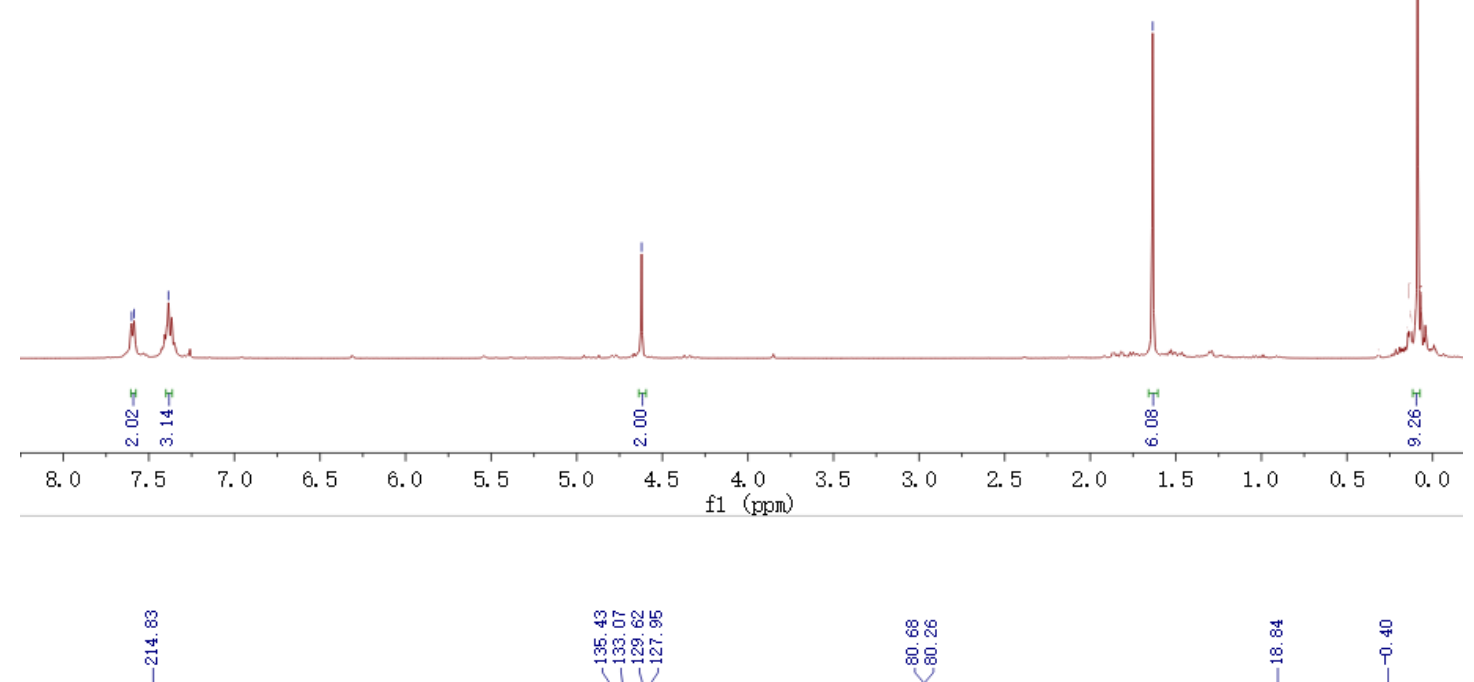

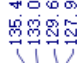

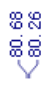

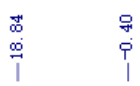

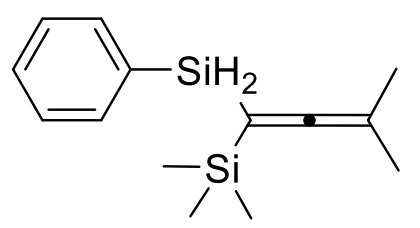

${ }^{13} \mathrm{C}$ NMR spectrum in $\mathrm{CDCl}_{3}$

$8 t$

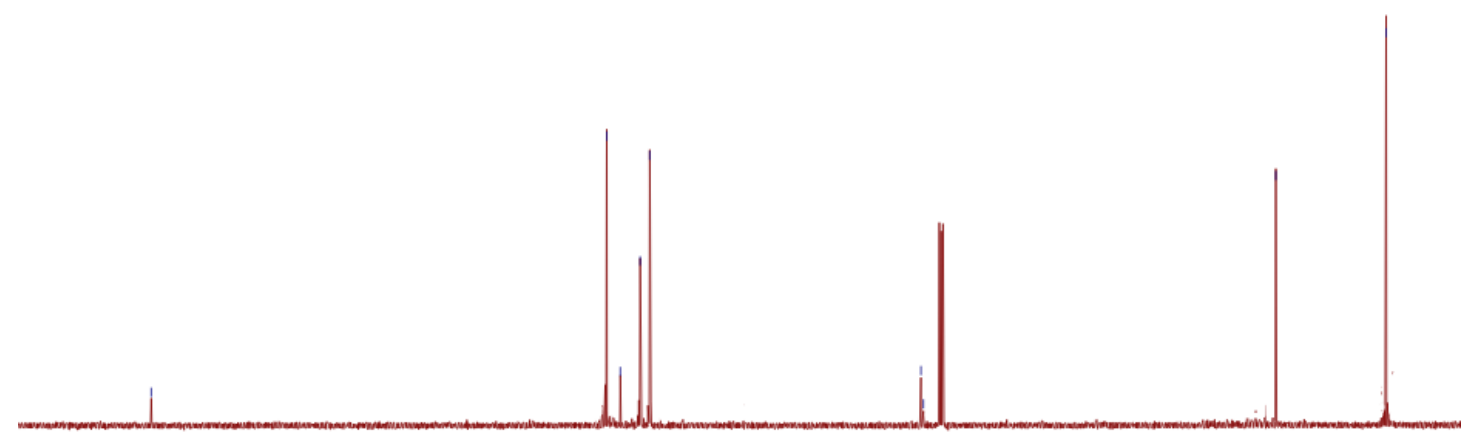

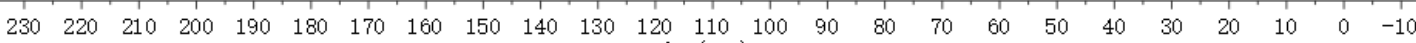
f1 (ppm) 


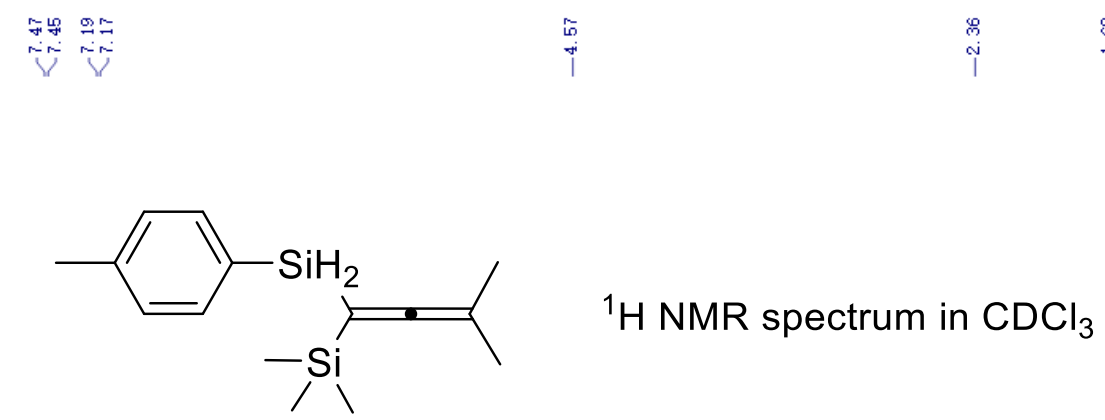

$8 u$

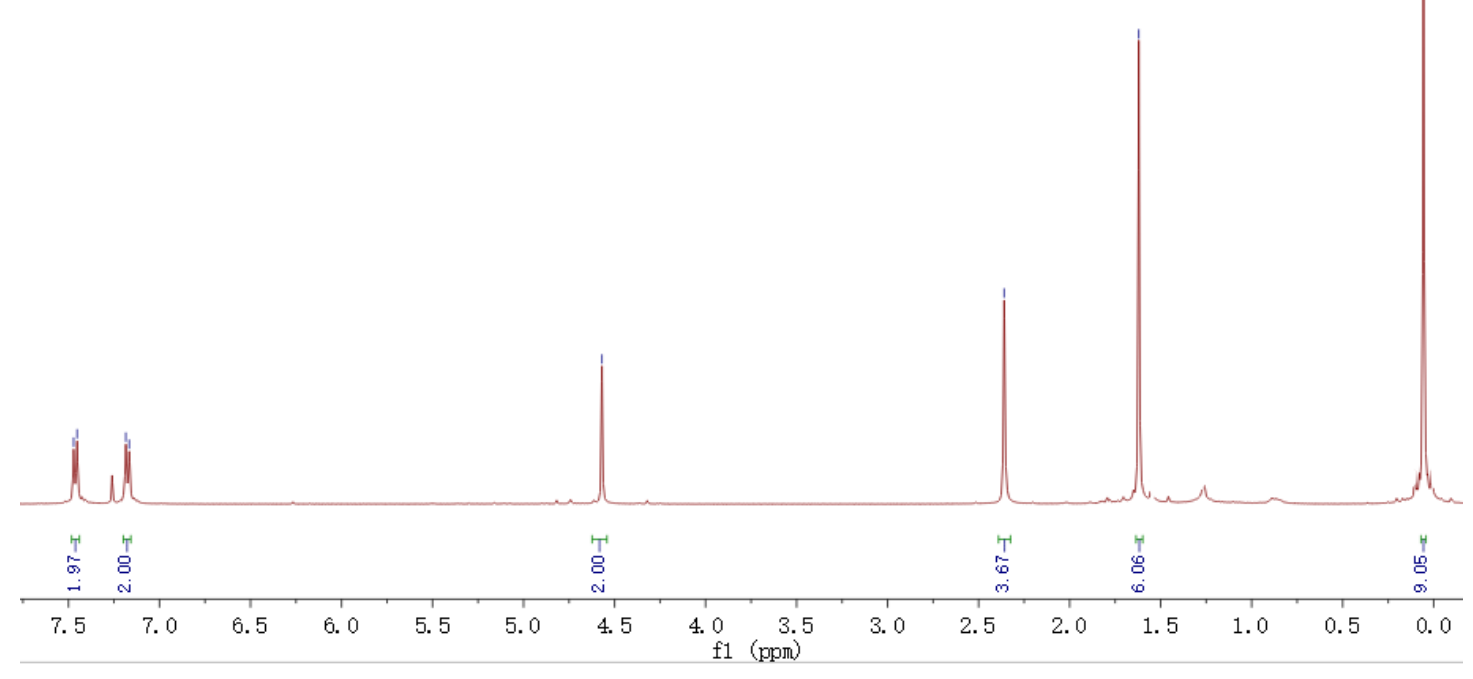

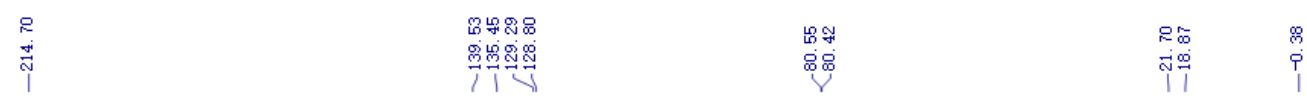

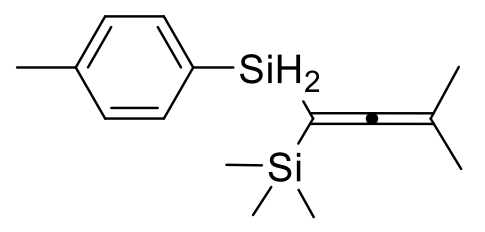

${ }^{13} \mathrm{C}$ NMR spectrum in $\mathrm{CDCl}_{3}$

$8 u$

$\begin{array}{llllllllllllllllllllllll}220 & 210 & 200 & 190 & 180 & 170 & 160 & 150 & 140 & 130 & 120 & 110 & 100 & 90 & 80 & 70 & 60 & 50 & 40 & 30 & 20 & 10 & 0 & -10\end{array}$ f1 (ppm) 
次

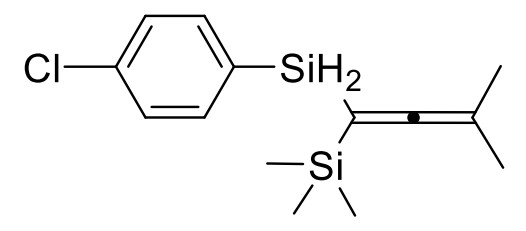

${ }^{1} \mathrm{H}$ NMR spectrum in $\mathrm{CDCl}_{3}$

$8 v$

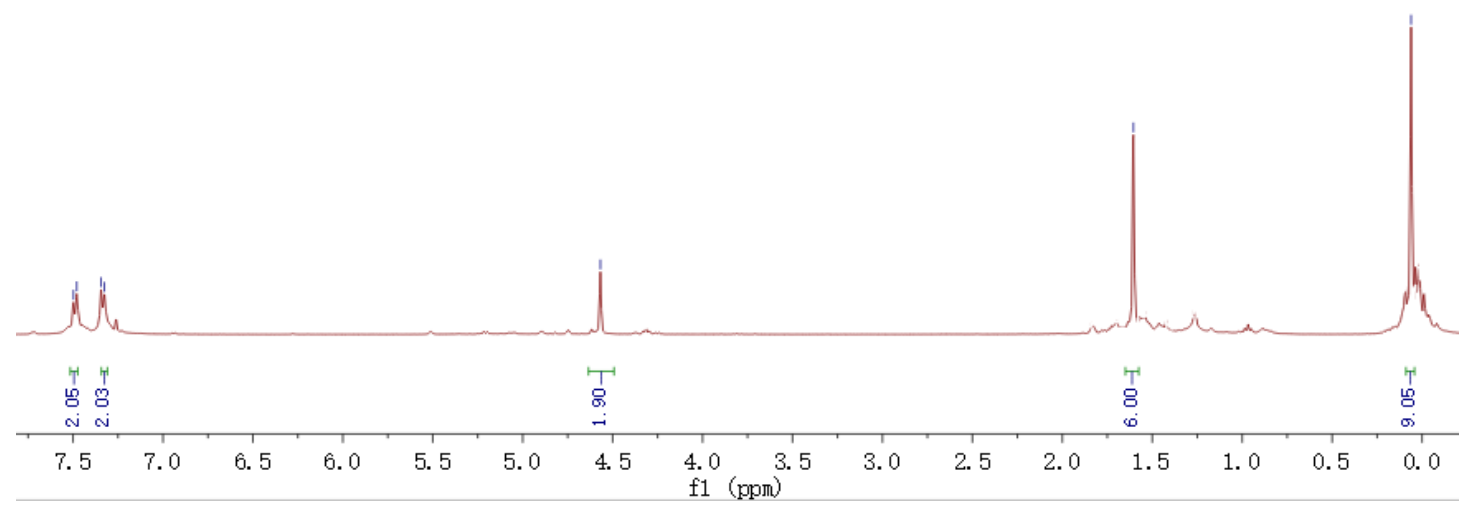

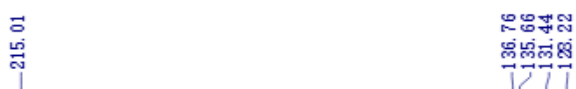

8\%

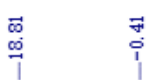

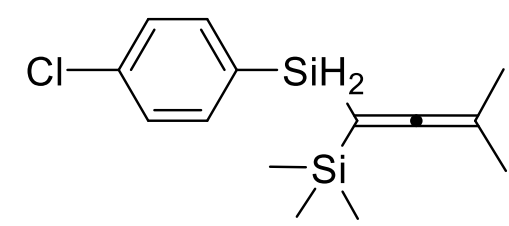

${ }^{13} \mathrm{C}$ NMR spectrum in $\mathrm{CDCl}_{3}$

$8 v$

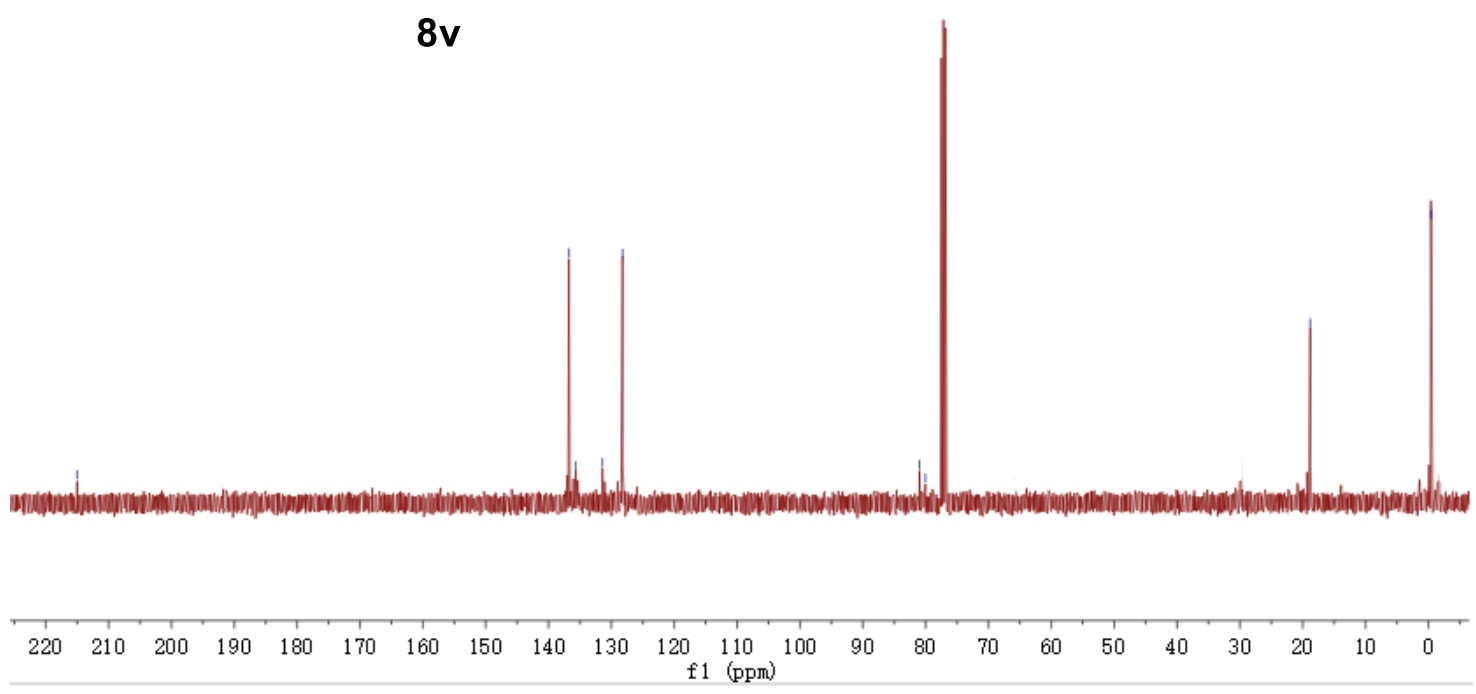




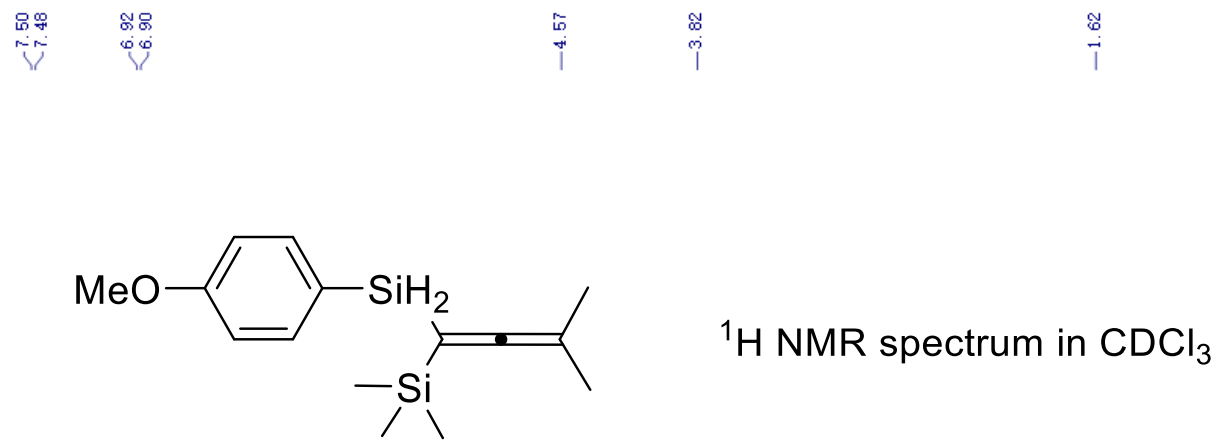

\section{$8 w$}

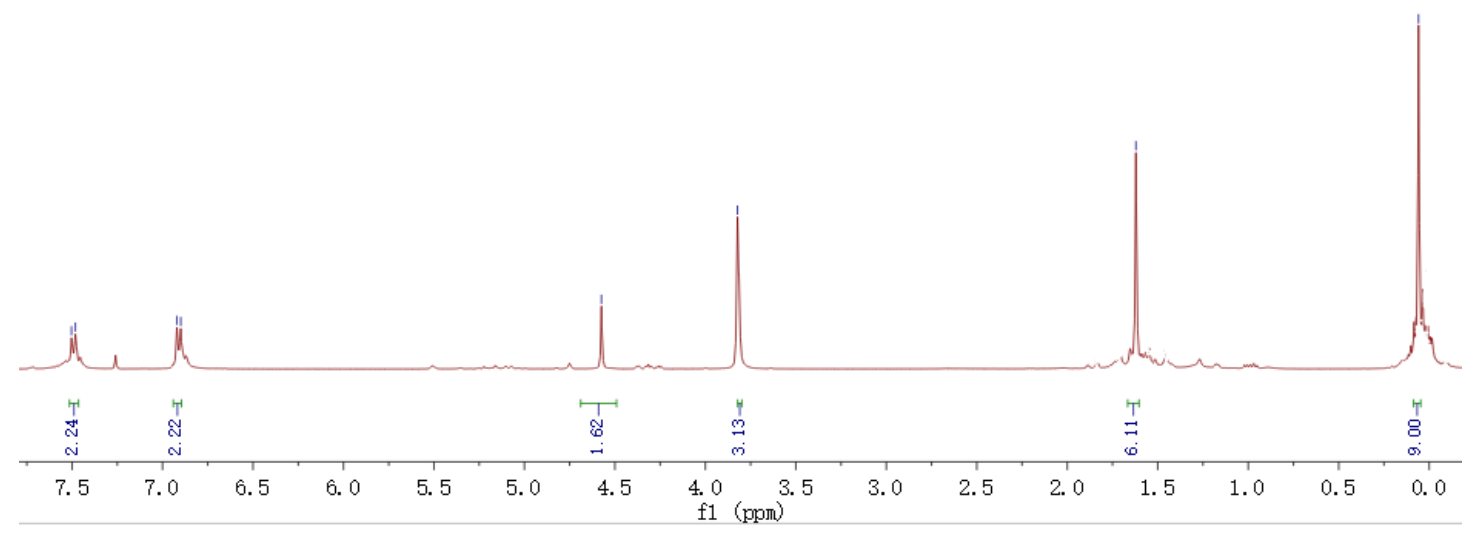

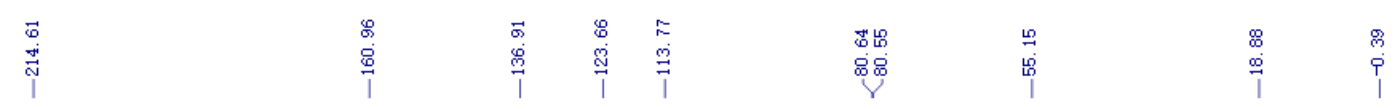

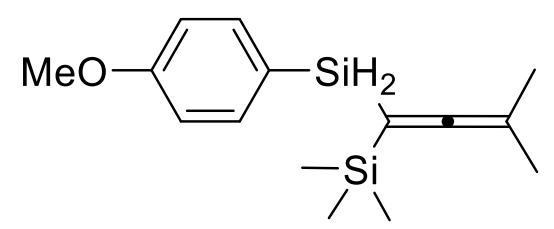

${ }^{13} \mathrm{C}$ NMR spectrum in $\mathrm{CDCl}_{3}$

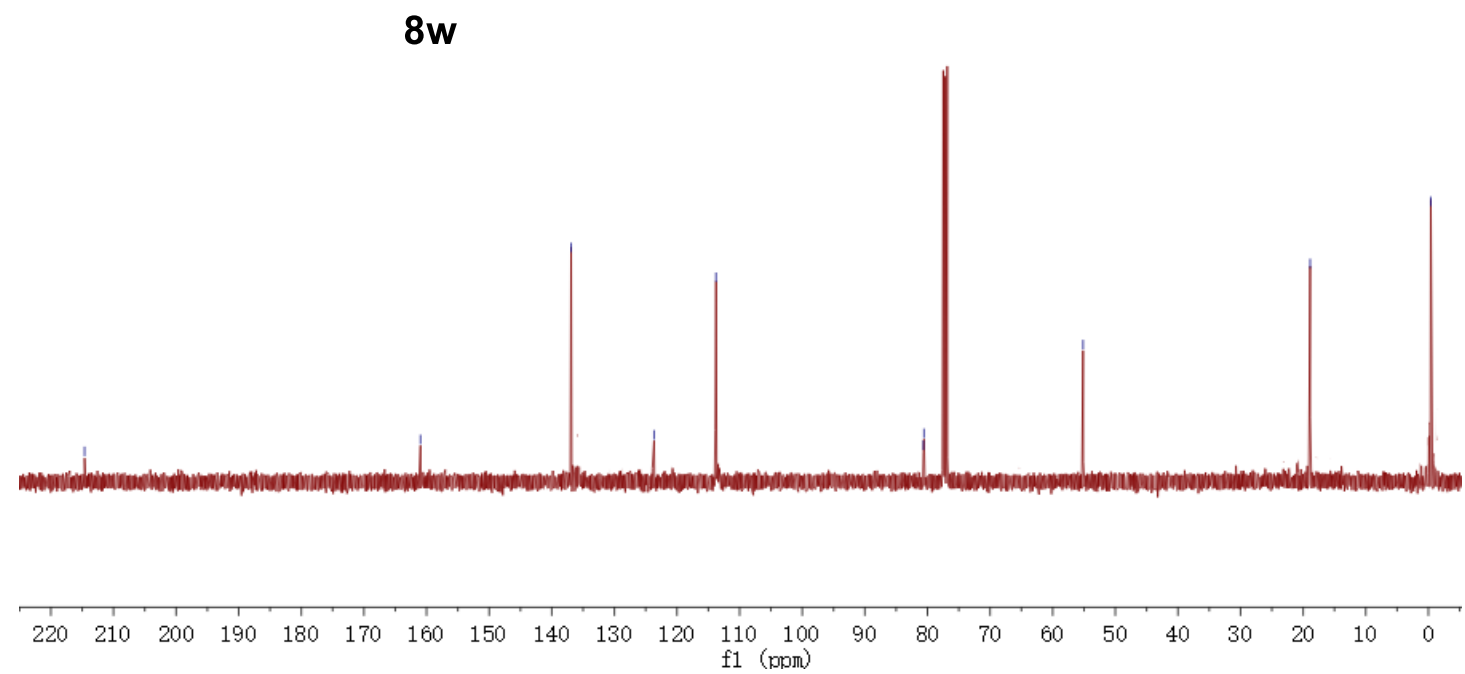




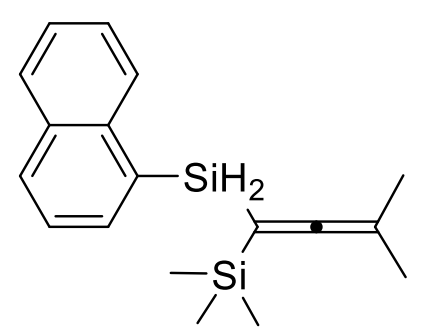

${ }^{1} \mathrm{H}$ NMR spectrum in $\mathrm{CDCl}_{3}$

$8 \mathrm{x}$

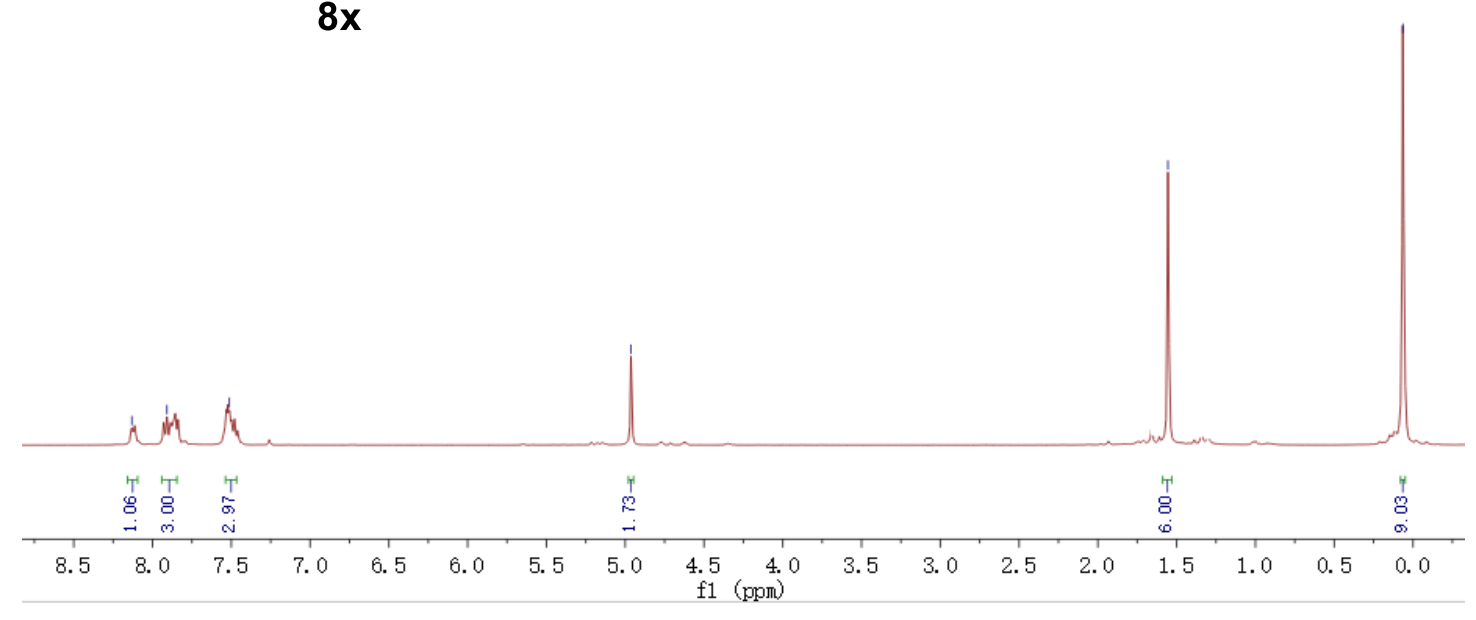

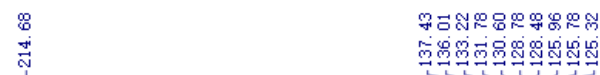

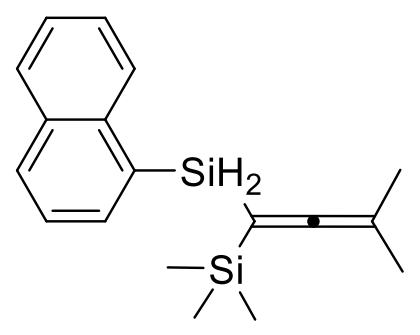

${ }^{13} \mathrm{C}$ NMR spectrum in $\mathrm{CDCl}_{3}$

$8 \mathrm{x}$

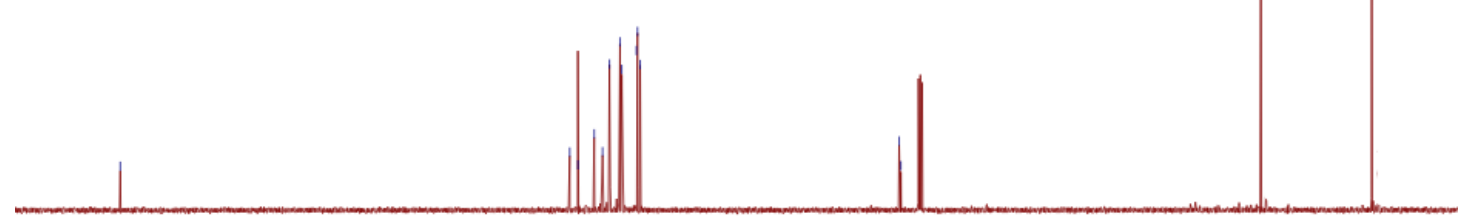

$\begin{array}{lllllllllllllllllllllllllll}230 & 220 & 210 & 200 & 190 & 180 & 170 & 160 & 150 & 140 & 130 & 120 & 110 & 100 & 90 & 80 & 70 & 60 & 50 & 40 & 30 & 20 & 10 & 0 & -10\end{array}$ f1 (ppm) 


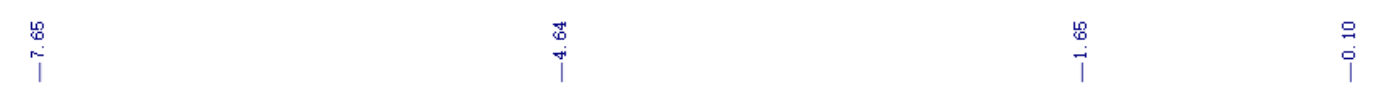

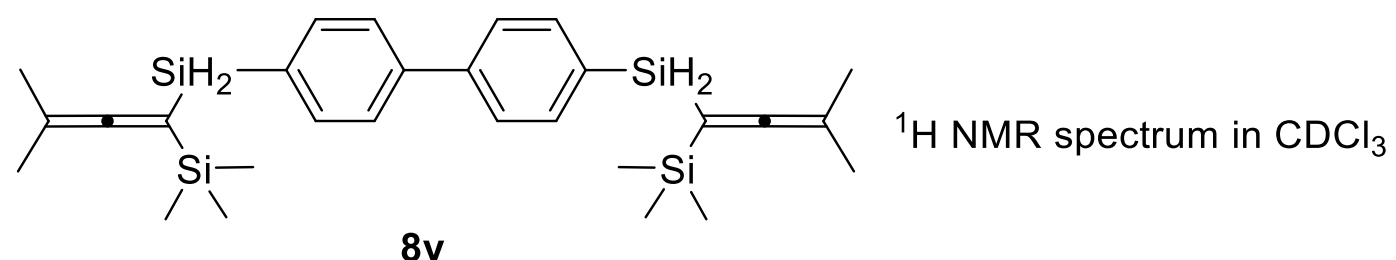

$8 y$

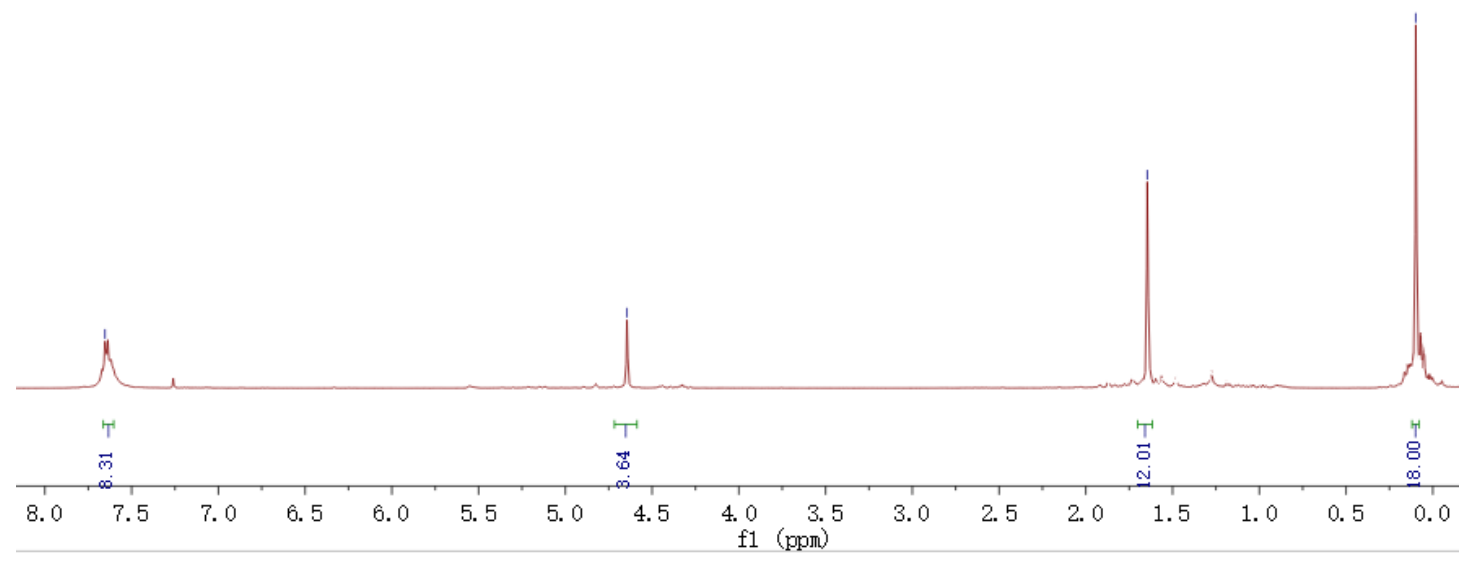

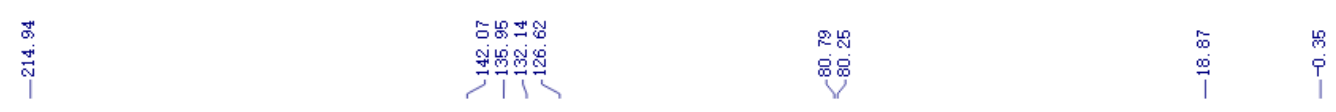

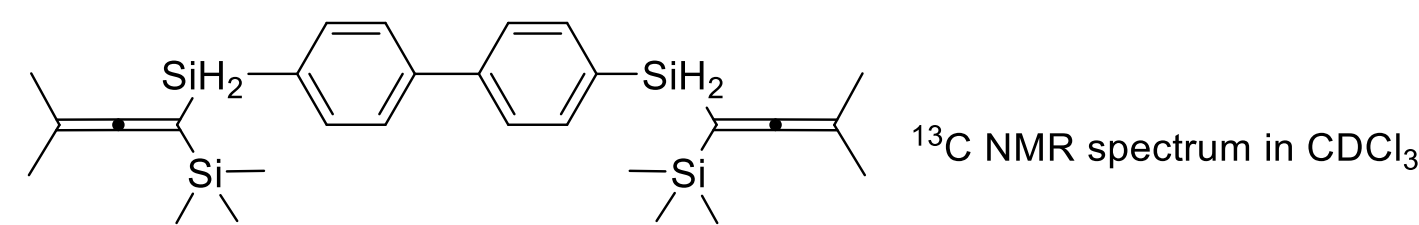

$8 y$

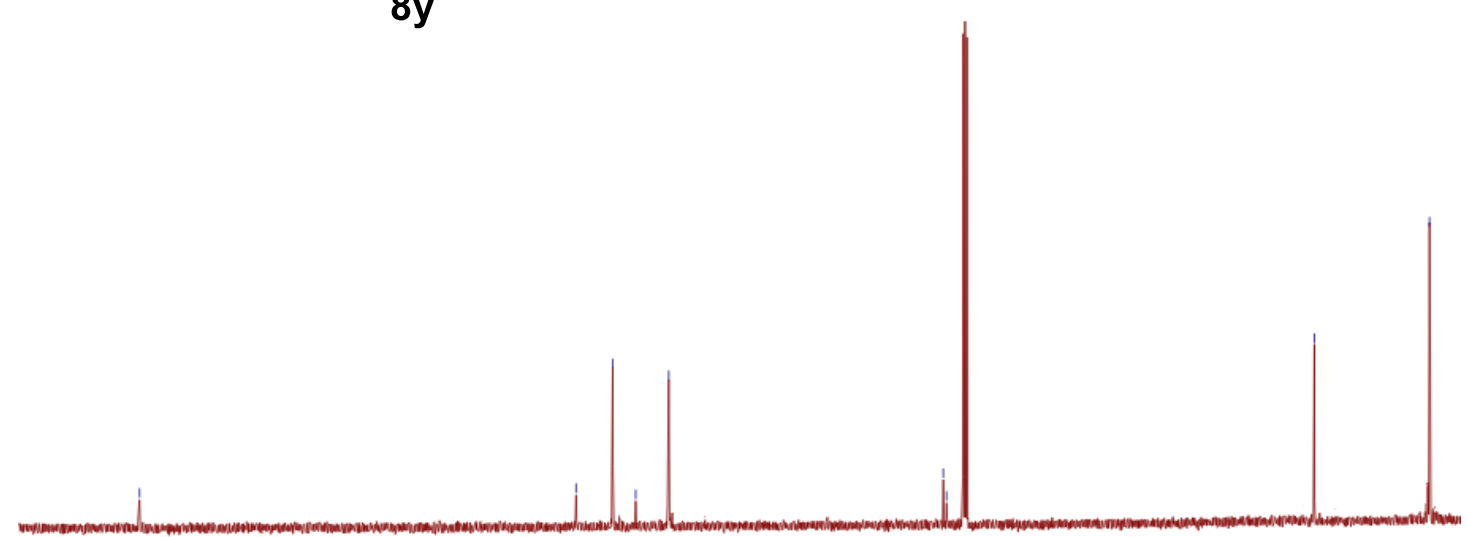

$\begin{array}{llllllllllllllllllllllllllllll}230 & 220 & 210 & 200 & 190 & 180 & 170 & 160 & 150 & 140 & 130 & 120 & 110 & 100 & 90 & 80 & 70 & 60 & 50 & 40 & 30 & 20 & 10 & 0\end{array}$ 


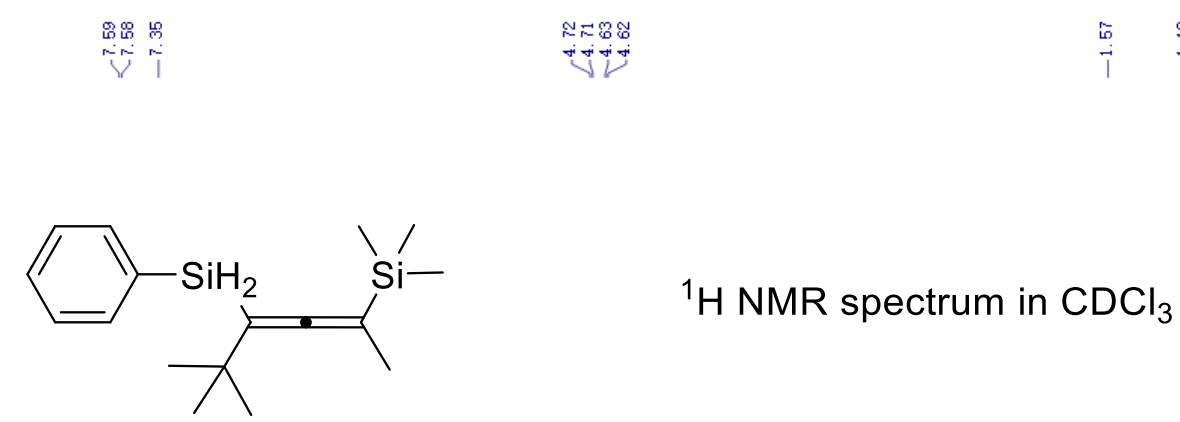

$8 z$
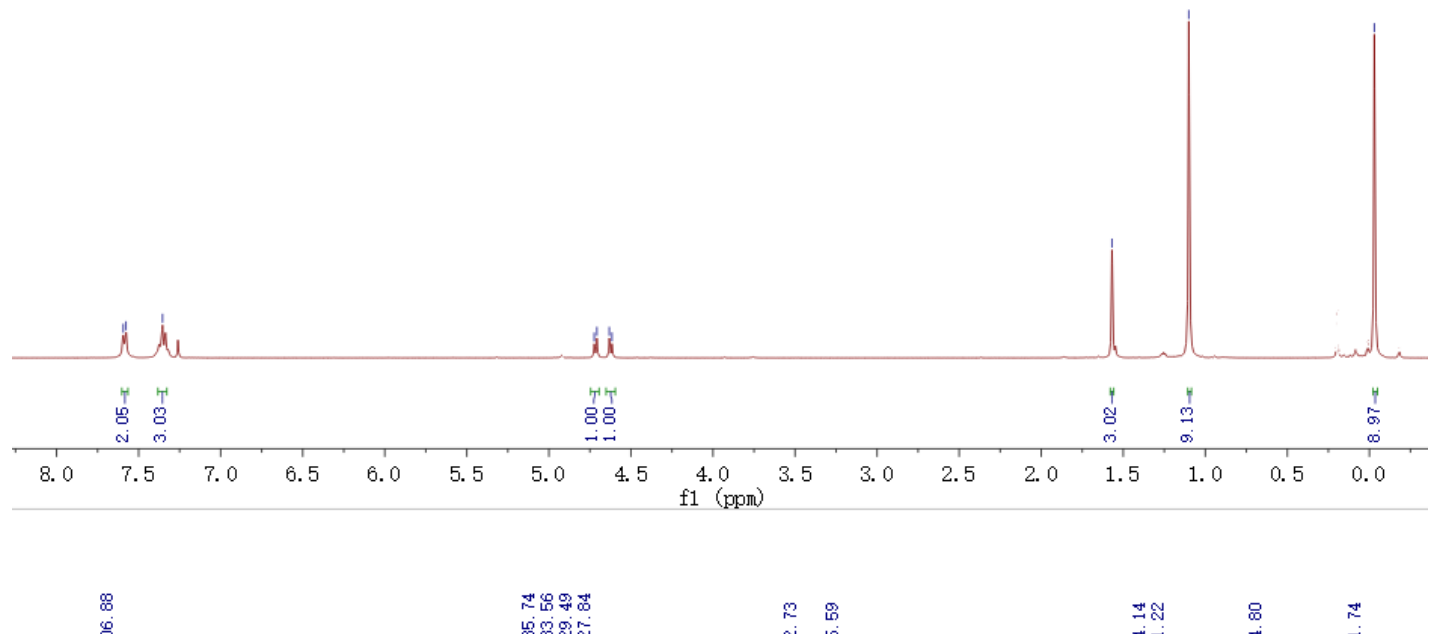

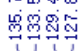

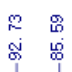

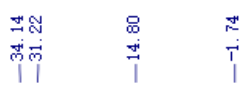

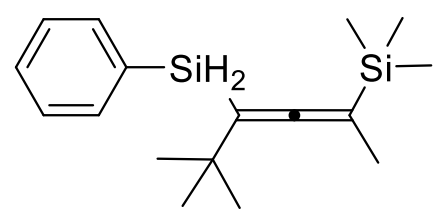

${ }^{13} \mathrm{C} \mathrm{NMR}$ spectrum in $\mathrm{CDCl}_{3}$

$8 z$

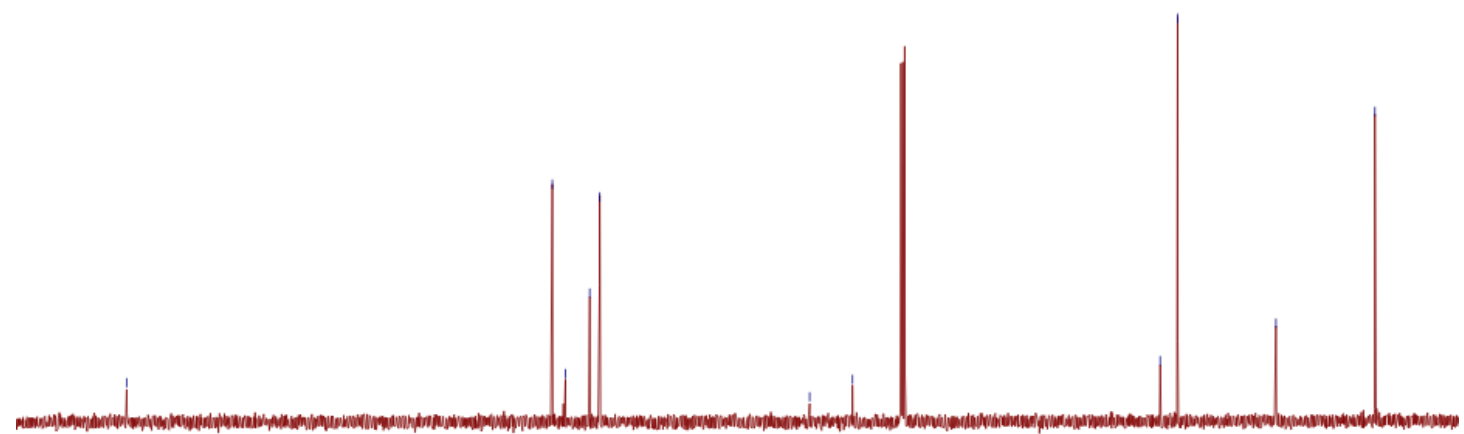

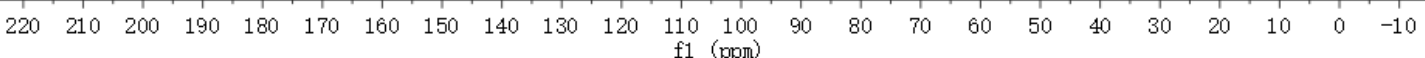




\section{果}

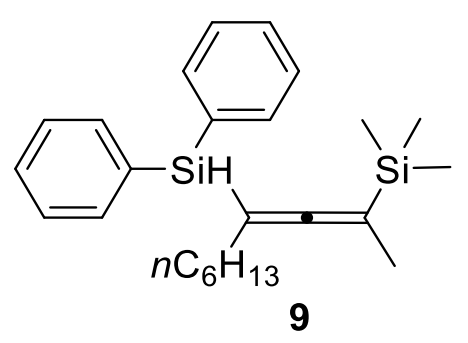

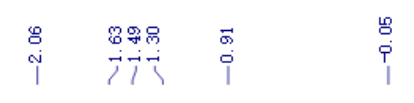

\section{${ }^{1} \mathrm{H}$ NMR spectrum in $\mathrm{CDCl}_{3}$}

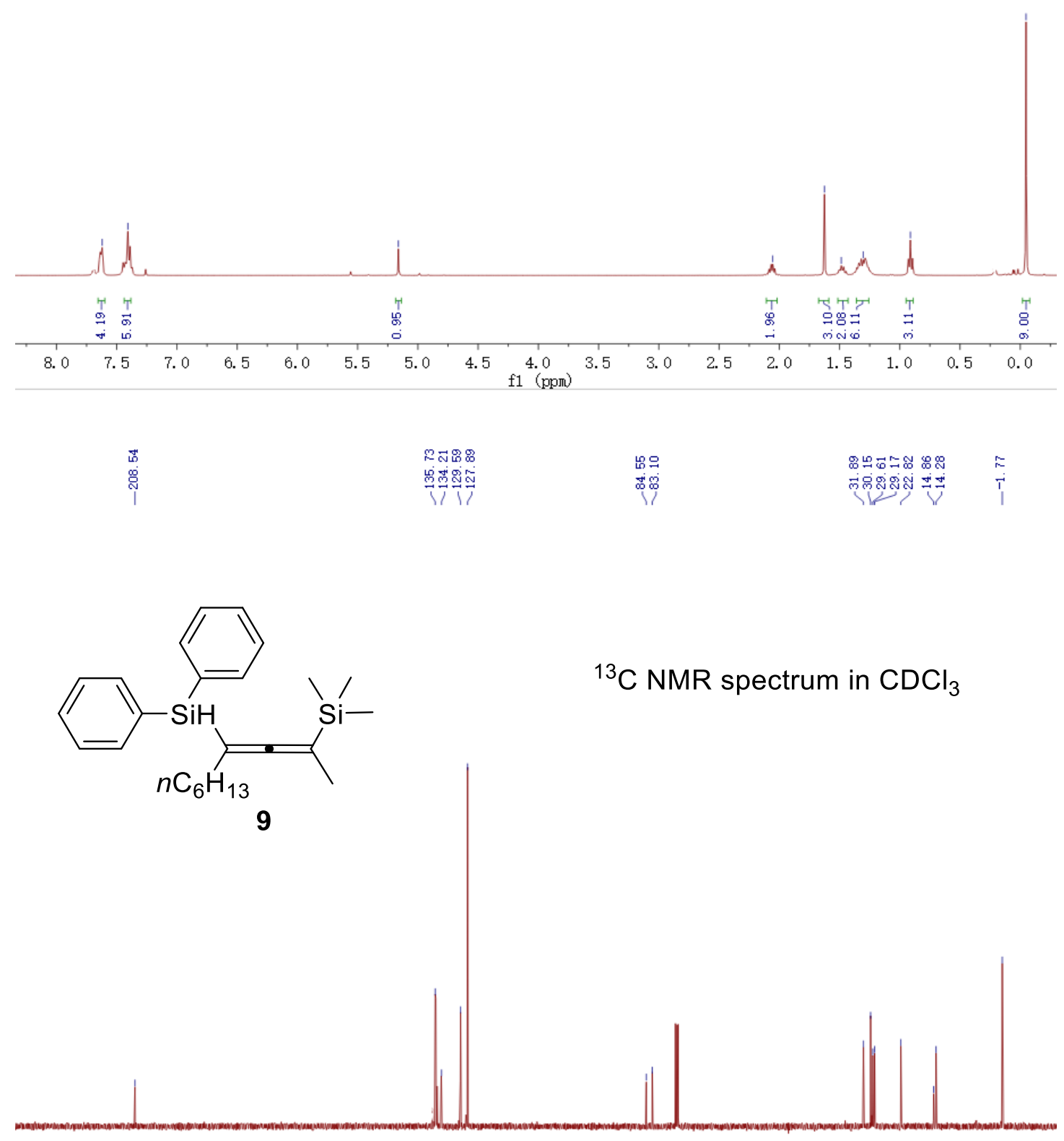

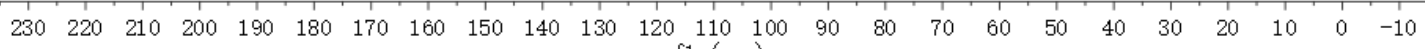
f1 (ppm) 

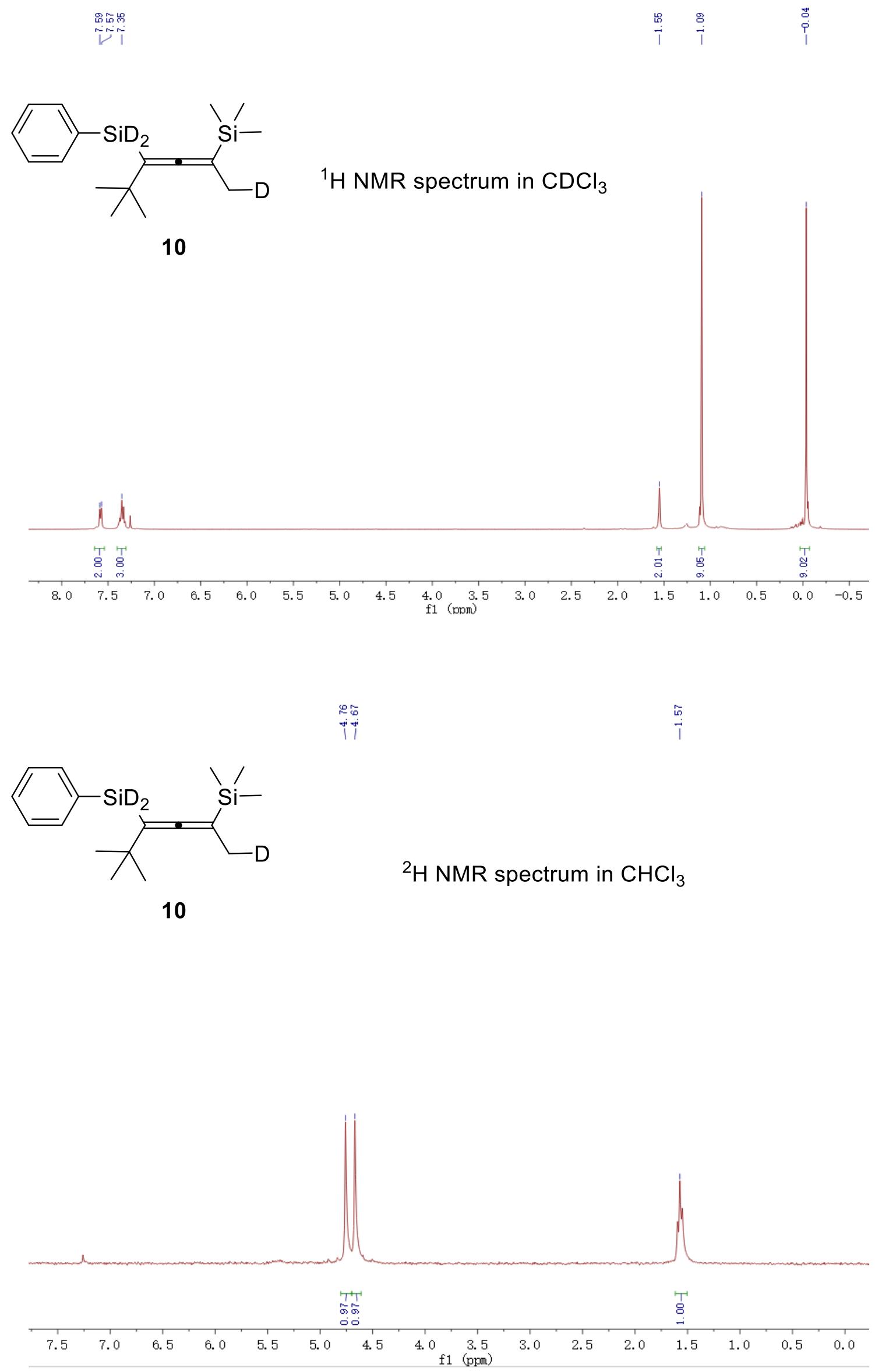


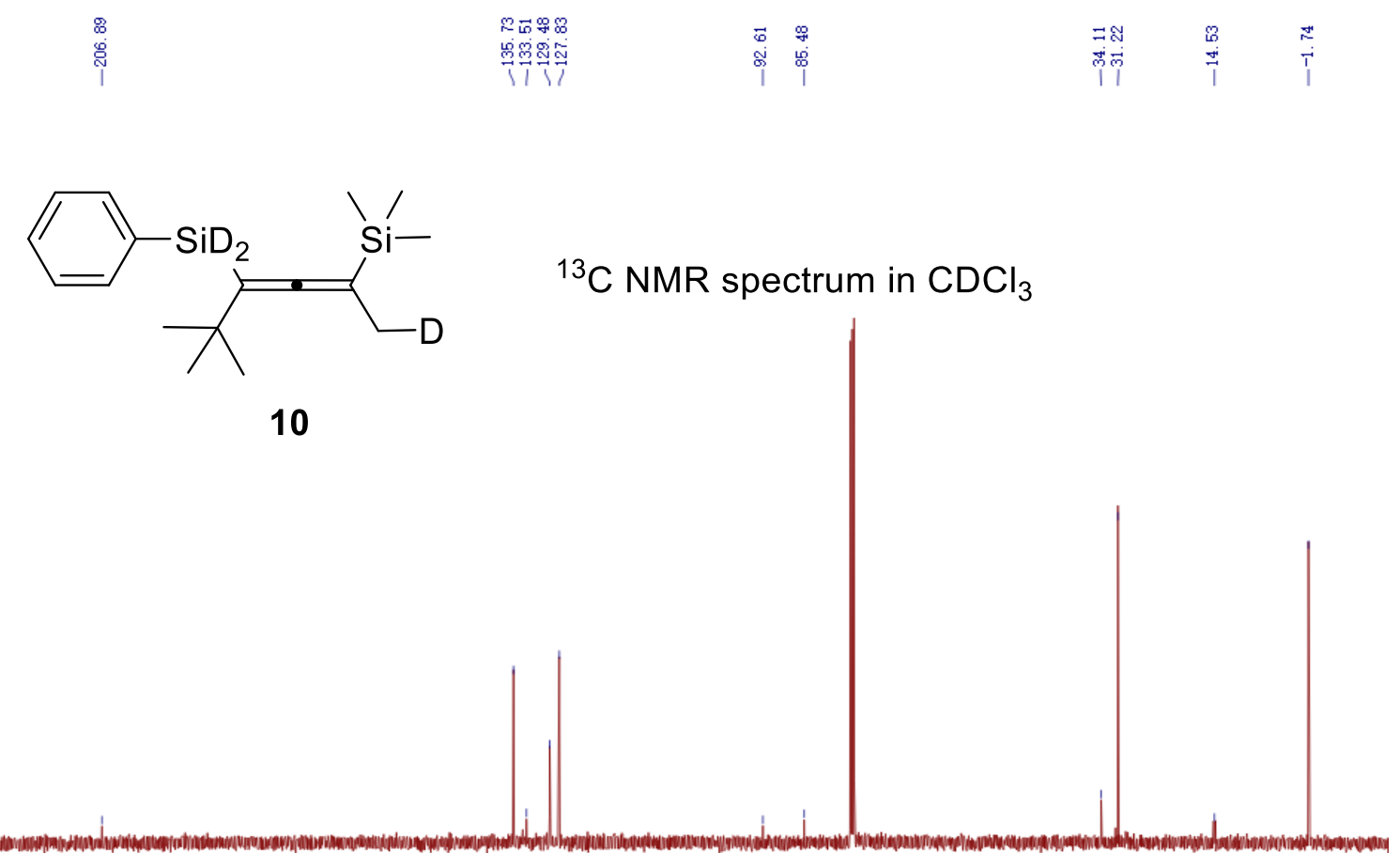

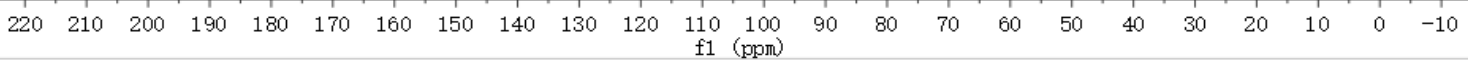




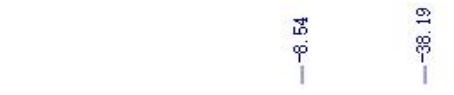

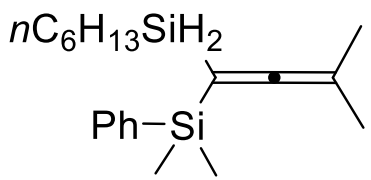

$8 d$
${ }^{29} \mathrm{Si} \mathrm{NMR}$ spectrum in $\mathrm{CDCl}_{3}$

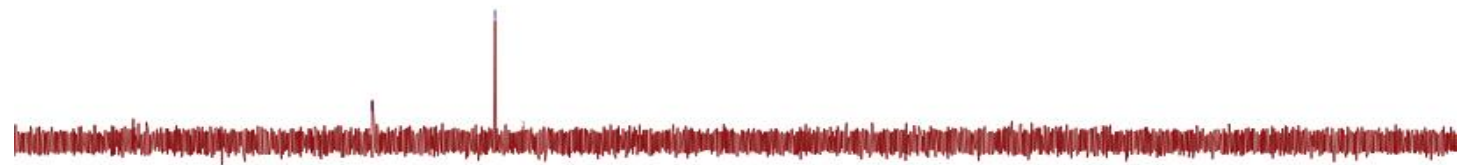

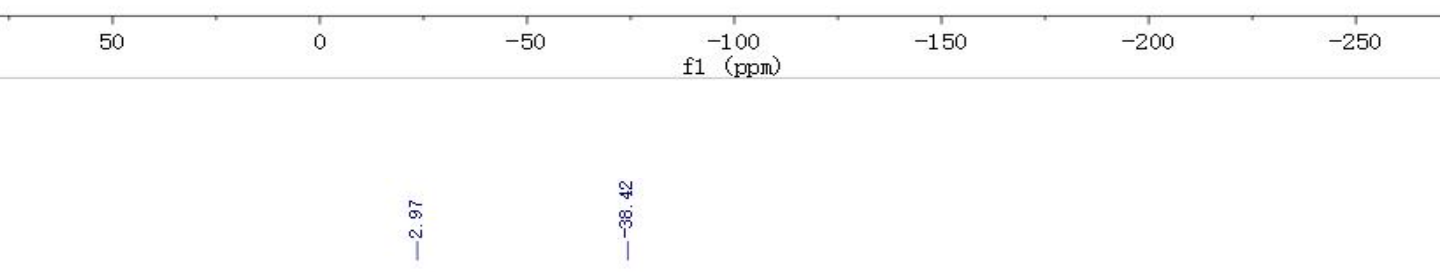

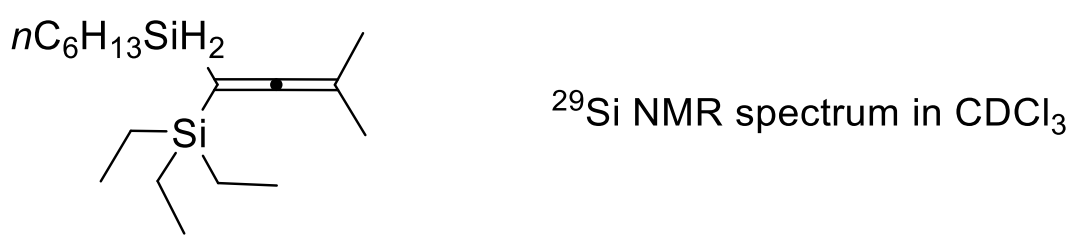

$8 e$

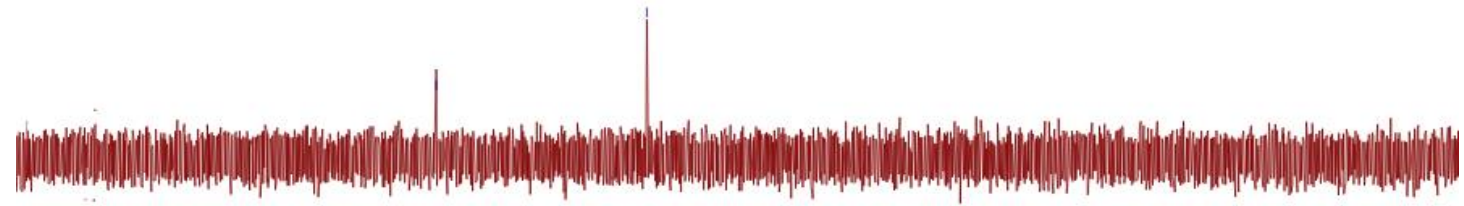

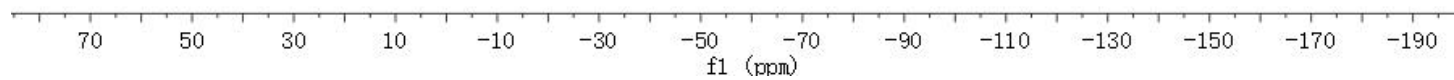




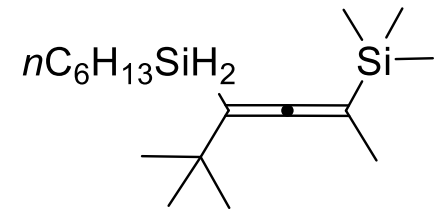

$8 h$
${ }^{29} \mathrm{Si} \mathrm{NMR} \mathrm{spectrum} \mathrm{in} \mathrm{CDCl}_{3}$

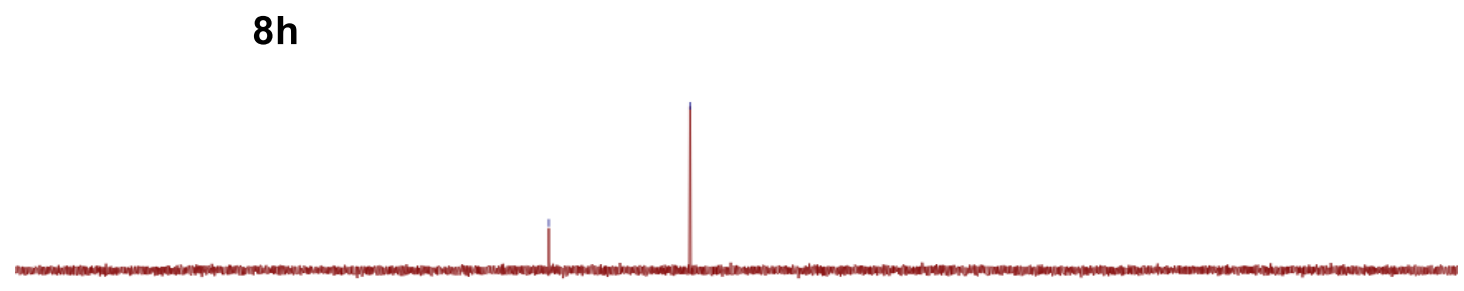

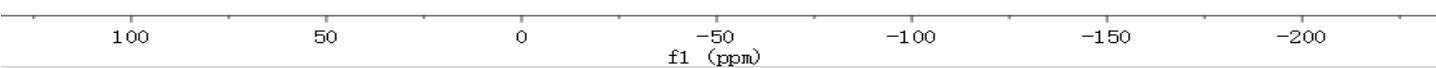

苦
i

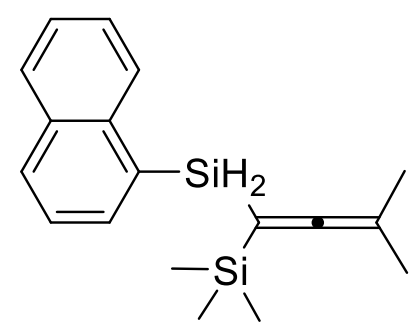

${ }^{29} \mathrm{Si} \mathrm{NMR} \mathrm{spectrum} \mathrm{in} \mathrm{CDCl}_{3}$

$8 x$

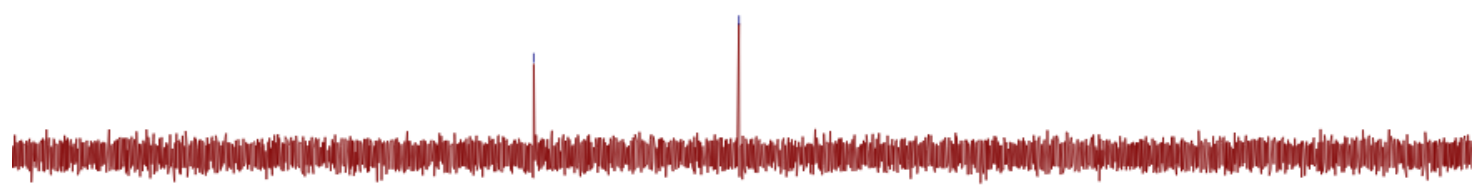

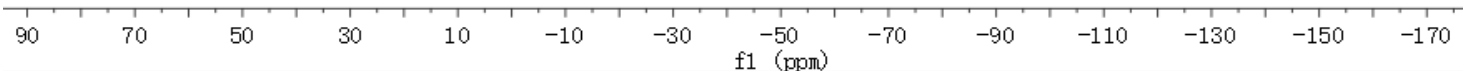




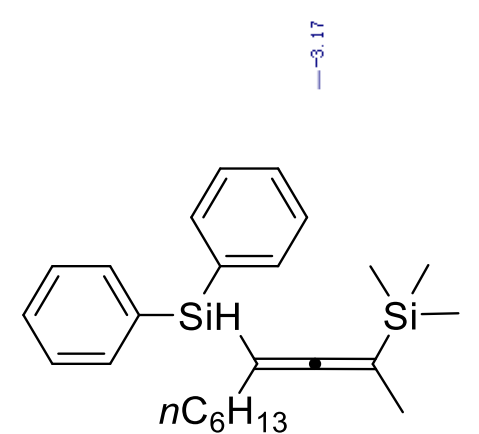

9

${ }^{29} \mathrm{Si} \mathrm{NMR}$ spectrum in $\mathrm{CDCl}_{3}$

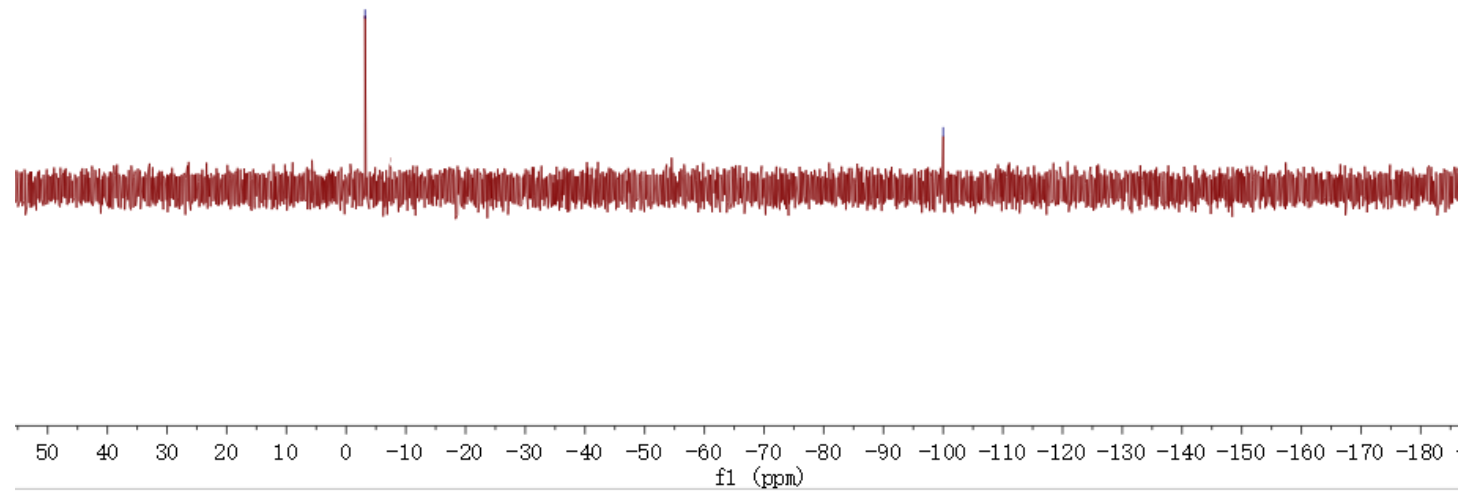




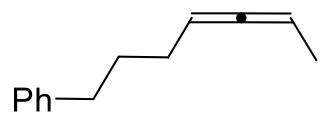

${ }^{1} \mathrm{H}$ NMR spectrum in $\mathrm{CDCl}_{3}$

11

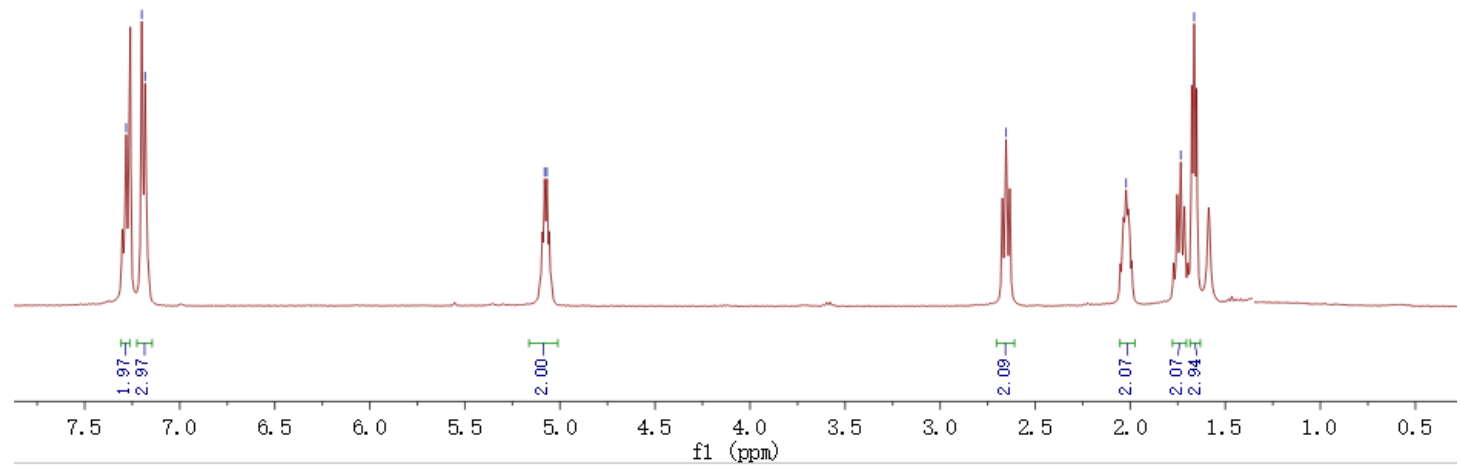

ìn

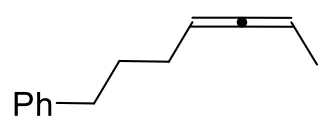

${ }^{13} \mathrm{C}$ NMR spectrum in $\mathrm{CDCl}_{3}$

11

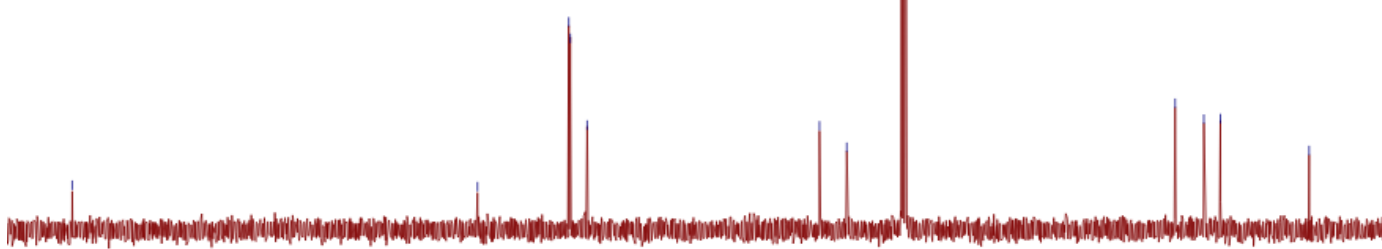

$\begin{array}{lllllllllllllllllllll}210 & 200 & 190 & 180 & 170 & 160 & 150 & 140 & 130 & 120 & 110 & 100 & 90 & 80 & 70 & 60 & 50 & 40 & 30 & 20 & 10\end{array}$ 


\section{IR Spectrum}

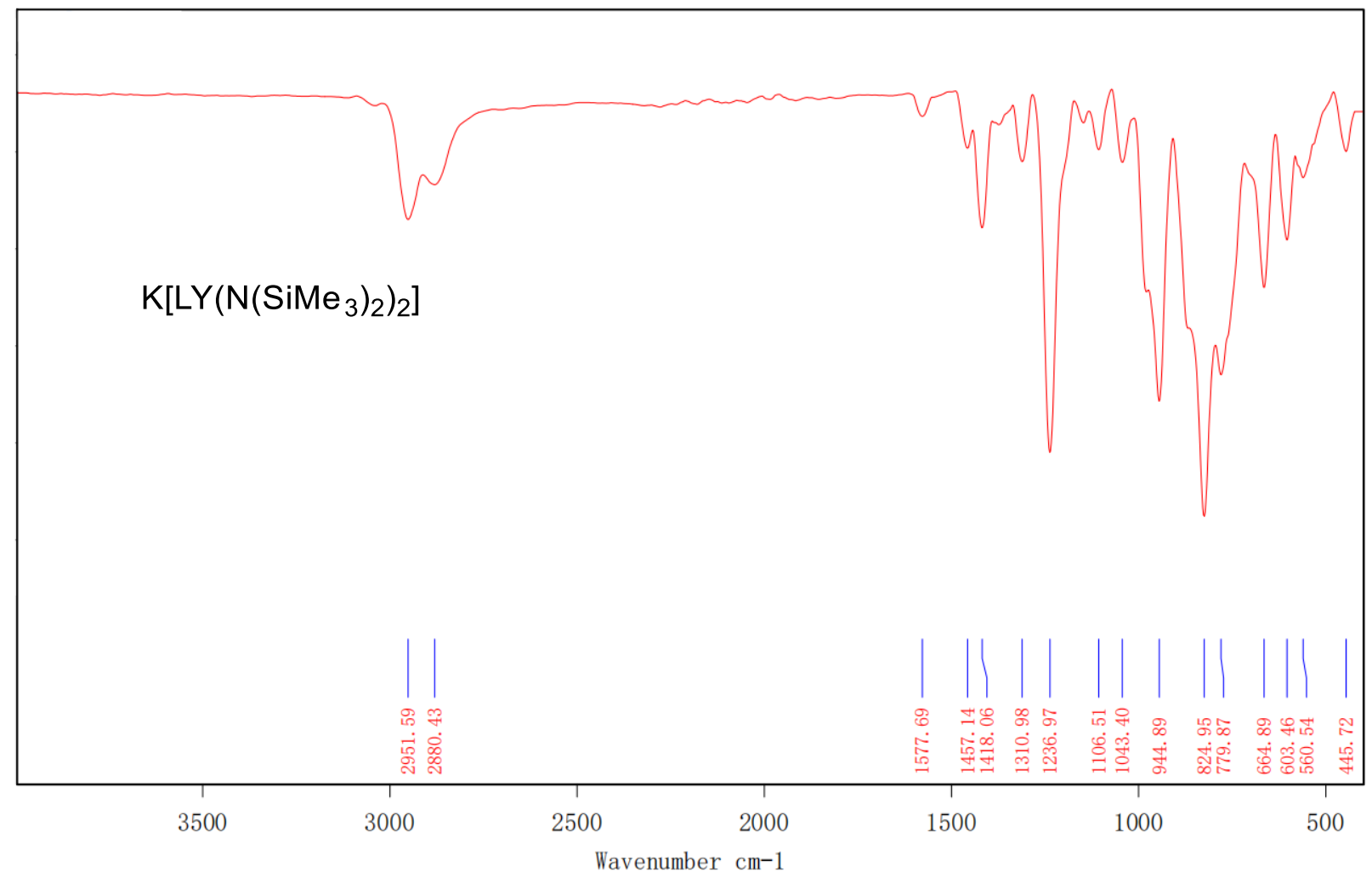

